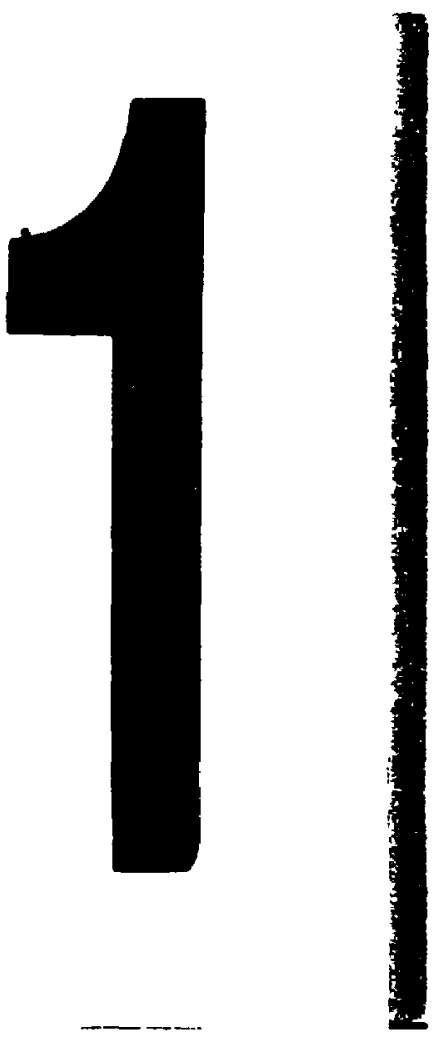

PM-1 3Y" $X 4$ " PHOTOGRAPHIC MICROCOPY TARGET NBS 1010a ANSI/1SO \#2 EQUIVALENT

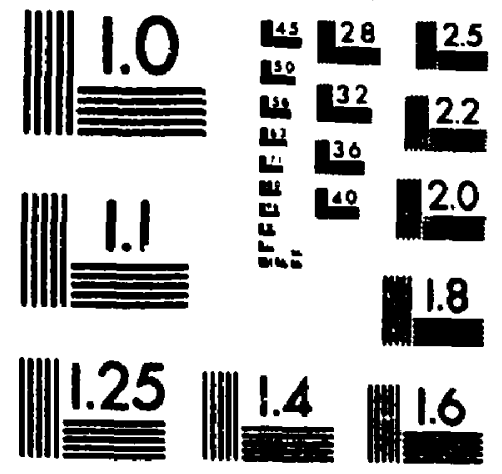

PRECISIONSM RESOLUTION TARGETS

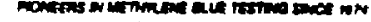

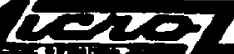

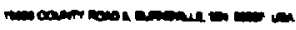

T. 
Acquisitions and

Bibliographic Services Branch

395 Wellington Strezt

Ottawa. Ontano

KIA ON4
Bibliotheque nationale

du Canada

Direction des acqul: rons et

des services bibliographıques

395, nue Wellington

Otrawa (Onlario)

KIA ON4
NOTICE

The quality of this microform is heavily dependent upon the quality of the original thesis submitted for microfilming. Every effort has been made to ensure the highest quality of reproduction possible.

If pages are missing, contact the university which granted the degree.

Some pages may have indistinct print especially if the original pages were typed with a poor typewriter ribbon or if the university sent us an inferior photocopy.
La qualité de cette microforme dépend grandement de la qualité de la thèse soumise au microfilmage. Nous avons tout fait pour assurer une qualité supérieure de reproduction.

S'il manque des pages, veuillez communiquer avec l'université qui a conféré le grade.

La qualité d'impression de certaines pages peut laisser à désirer, surtout si les pages originales ont été dactylographiées à l'aide d'un ruban usé ou si l'université nous a fait parvenir une photocopie de qualité inférieure.

La reproduction, même partielle, de cette microforme est soumise à la Loi canadienne sur le droit d'auteur, SRC 1970, c. C-30, ot ses amendements subséquents.
Reproduction in full or in part of this microform is governed by the Canadian Copyright Act, R.S.C. 1970, c. C-30, and subsequent amendments.

AVIS 


\title{
THIN-WALLED BOX BEAM FINITE ELEMENTS FOR STATIC ANALYSIS OF CURVED HAUNCHED AND SKEW MUITICELL BOX GIRDER BRIDGES
}

\author{
By \\ Hangang I.i \\ B.Sc., M.Sc. (Civil)
}

\author{
A thesis submitted to \\ the Faculty of Graduate Studies and Research \\ in partial fulfillment of the requirements \\ for the degree of
}

DOCTOR OF PHILOSOPHY

DEPARTMENT OF CIVIL ENGINEERING

CARLETON UNIVERSITY

OTTAWA, CANADA

May, 1992.

(C) 1992

Hangang $\mathrm{Li}$ 
Acquisitions and

Biblıgraphic Services Branch

395 Wellington Street

Ottawa. Ontarvo

KIA ON4
Direction des acquisitions et

des services bibliographiques

395. nue Wellington

Ottawa (Ontario)

KIA ON4
The author has granted an irrevocable non-exclusive licence allowing the National Library of Canada to reproduce, loan, distribute or sell copies of his/her thesis by any means and in any form or format, making this thesis available to interested persons.
L'auteur a accordé une licence irrévocable et non exclusive permettant à la Bibliotheque nationale du Canada de reproduire, prêter, distribuer ou vendre des copies de sa thèse de quelque manière et sous quelque forme que ce soit pour mettre des exemplaires de cette thèse à la disposition des personnes intéressées.
The author retains ownership of the copyright in his/her thesis. Neither the thesis nor substantial extracts from it may be printed or otherwise reproduced without his/her permission.
L'auteur conserve la propriété du droit d'auteur qui protège sa thèse. Ni la thèse ni des extraits substantiels de celle-ci ne doivent être imprimés ou autrement reproduits sans son autorisation. 
The undersigned hereby recommend to the

Faculty of Graduate Studies and Research

acceptance of the thesis entitled

"Thin-Walled Box Beam Finite Elements

for Static Analysis of Curved

Haunched and Skew Multicell

Box Girder Bridges"

submitted by

Hangang Li

in partial fulfilment of the requirements for the degree of

Doctor of Philosophy

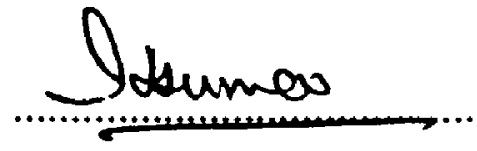

Chairman, Department of Civil Engineering

J.L. Humar

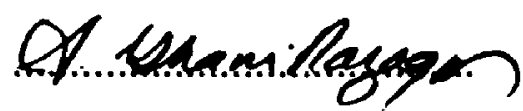

Thesis Supervisor

A.G. Razaqpur

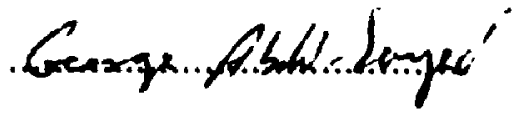

External Examiner

G. Abdel-Sayed

Carleton University

June, 1992 


\section{Abstract}

Two efficient thin-walled box beam finite elements which can model extension. flexure, torsion, torsional warping, distortion, distortional warping and shear lag effects are developed using an extended version of Vlasov's thin-walled beam theory. The elements can be used for the analysis of multicell box girder bridges with genrral geometry.

For straight right multicell box girder structures, an orthogonalization procedure is employed to uncouple the various distortional and shear lag modes. The governing differential equation pertaining to each action is used to derive the exact shupe functions and the stiffness matrix and nodal load vector of the element. This exact shape function finite element model eliminates the need for dividing the bridge into many elements in each span. For curved box beam finite element, geometry mapping functions and the conventional polynomial shape functions are used to model bridges of general geometry, such as haunched and skew bridges. The application of the two elements is subsequently extended to the analysis of box girder assemblages. Considering thin-walled beam theory, the effects of prestressing, temperature, creep and shrinkage are also included in the analysis.

Finally, the two elements are used to perform linear elastic analysis of an extensive range of box girder bridges, the results of which are compared with other alternative numerical methods, such as shell finite element and finite strip method, and published experimental or prototype measurements. In practically all cases the results agree closely with other advanced methods of analysis. The advantuges of the proposed method are its efficiency, ease of application, and the transparent way in which it deals with the different structural actions.

Two computer programs, SBOXEF and CBOX, which contain the proposed elements and method of analysis, are written in FORTRAN 77 and they run on either Apollo or SUN workstations. 


\section{Acknowledgments}

The Author wishes to express his thanks to Professor A.G. Razaqpur, under whose supervision this research work was carried ont, for his able guidance, understanding, and encouragement.

Special thanks are due to Professor J.L. Humar and Professor S.J. Kennedy for the time given to the author for many fruitful discussions on the analysis.

Thanks are also due to the late Dr. B.I. Maisel of Cement and Concrete Association in London, England for providing us with literature containing his extensive work related to the subject of the present study.

To author's numerous friends and colleagues, who have not been mentioned and who helped and extended moral support durinng this study, I express my appreciation.

The scholarship provided to the author by Department of Civil Engineering, Carleton University is gratefully acknowledged.

Finally (but certainly not least), the love, support and encouragement of the author's parents and wife have played a major role in making it possible to complete this study. 


\section{TABLE OF CONTENTS}

ABSTRACT . . . . . . . . . . . . . . ii

ACKNOWLEDGMENTS . . . . . . . . . . . . . . . . . iii

TABLE OF CONTENTS . . . . . . . . . . . . . . . . . . . . . iv

LIST OF FIGURES . . . . . . . . . . . . . . . . . . . . . . . viii

LIST OF IMPORTANT NOTATIONS . . . . . . . . . . . . . . xi

1 INTRODUCTION ..................... 1

1.1 Statement of the Problem . . . . . . . . . . . . . . . . . . . 1

1.2 Previous ïork . . . . . . . . . . . . . . . . . . . . . . . . . 2

1.3 Objectives and Scope . . . . . . . . . . . . . . . . . . . . . . 7

2 BASIC THEORY OF THIN-WALLED BOX BEAMS . . . . . . 9

2.1 General . . . . . . . . . . . . . . . . . . . 9

2.2 Longitudinal Bending . . . . . . . . . . . . . . . . . . . . . 9

2.3 Shear Lag . . . . . . . . . . . . . . . . . . . 13

2.4 Torsion . . . . . . . . . . . . . . . . . . . . . . . . . . 19

2.4.1 Pure Torsion . . . . . . . . . . . . . . . . . . . . . . . 19

2.4.2 Torsional Warping . . . . . . . . . . . . . . . . . . . 22

2.5 Distortion . . . . . . . . . . . . . . . . . . . . . . . . 26

2.5.1 Distortion of a Single-Cell Beam . . . . . . . . . . . . . . 26

2.5.2 Distortı.on of Multicell Box Beams . . . . . . . . . . . . . 34

2.5.3 Transverse Bending . . . . . . . . . . . . . . . . . . . . 37

2.6 Summary of Governing Equaticns of Multicell Box Beams . . . . 37

3 STRAIGHT MULTICELL BEAM ELEMENT WITH

EXACT SHAPE FUNCTIONS . . . . . . . . . . . . . . . . . . . . . . 41

3.1 General . . . . . . . . . . . . . . . . . . 41

3.2 Basics of Displacement Method of Finite Elements . . . . . . . . 42 
3.3 Uncoupling the Governing Equations . . . . . . . . . . . . . . 43

3.3.1 Uncoupling the Distortional and Shear Lag Modes from the Non-Distortional Modes . . . . . . . . . . . . . . . 44

3.3.2 Uncoupling the Distortional and Shear Lag Modes . . . : . 46

3.4 Exact Solution of Uncoupled Governing Equations . . . . . . . . 48

3.4.1 Extension . . . . . . . . . . . . . . . . . . . . . . . . 48

3.4.2Flexure . . . . . . . . . . . . . . . . . . . . 49

3.4.3 Torsion and Shear Lag . . . . . . . . . . . . . . . . . . 49

3.4.4 Distortion . . . . . . . . . . . . . . . . . . . . 51

3.5 Finite Element Formulation . . . . . . . . . . . . . . . . . 56

3.5.1 Element Geometry and Coordinate System . . . . . . . . . 56

3.5.2Stress-Strain Relation . . . . . . . . . . . . . . . . . . . 57

3.5.3Stiffness Matrix and Equivalent Nodal Force Vector . . . . 58

4 VARIABLE DEPTH CURVED ELEMENT _. . . . . . . . . 72

4.1 General . . . . . . . . . . . . . . . . . . . . . 72

4.2 Basic Theory of Curved Thin-Walled Beams . . . . . . . . . . . 73

4.2.1 Flexure and Torsion . . . . . . . . . . . . . . . . . . 73

4.2.2Shear Lag . . . . . . . . . . . . . . . . . . . . . . . . 78

4.2.3 Distortion . . . . . . . . . . . . . . . . . . 82

4.3 Generalized Vlasov's Theory for Curved Multicell Box Bearns . . . 84

4.4 Finite Element Formulation . . . . . . . . . . . . . . . . . . 91

4.4.1 Element Geometry and Coordinate System . . . . . . . . . 91

4.4.2Shape Functions . . . . . . . . . . . . . . . . . . . . . . . . 94

4.4.3Stiffness Matrix and Equivalent Nodal Loads . . . . . . . . 95

4.4.4 Boundary Conditions . . . . . . . . . . . . . . . . . . . 96

4.5 Multicell Box Girder Assemblages . . . . . . . . . . . . . . . 97

5 PRESTRESSING, TEMPERATURE, CREEP AND SHRINKAGE EFFECTS . . . . . . . . . . . . . . . . . . . . 104

5.1 General . . . . . . . . . . . . . . . . . . . . 104

5.2 Prestressing Bar Element . . . . . . . . . . . . . . . . . 105 
5.3 Effects of Temperature . . . . . . . . . . . . . . . . . . . . 107

5.3.1 Temperature Distrihution in Box Girder Bridges . . . . . . 108

5.3.2 Nodal Forces and Thermal Stresses . . . . . . . . . . . . 110

5.4 Time-Dependent Effects . . . . . . . . . . . . . . . . . . . 113

5.4.1Creep . . . . . . . . . . . . . . . . . . . . . . 114

5.4.2Shrinkage ................ . 116

5.4.3 Time-Dependent Analysis . . . . . . . . . . . . . . . . 116

6 NUMERICAL EXAMPLES ................... . 124

6.1 General . . . . . . . . . . . . . . . . . . . . . . . 124

6.2 Straight Multicell Box Girder Bridges . . . . . . . . . . . . . . 125

6.2.1 Single-Cell Single Span Box Girder Bridge . . . . . . . . . 125

6.2.2 Two-Cell Two Span Box Girder Bridge . . . . . . . . . . . 131

6.2.3 Three-Cell Single Span Box Girder Bridge . . . . . . . . . 130

6.2.4 Two-Spine Single Span Box Girder Bridge . . . . . . . . . 141

6.3 Curved Box Girder Bridges . . . . . . . . . . . . . . . . . . . 144

6.3.1 Singie-Cell Straight Box Girder Bridges . . . . . . . . . . 144

6.3.2 Single-Cell Haunched Box Beams . . . . . . . . . . . . . 140

6.3.3 Two-Cell Skewed Box Girder Bridge . . . . . . . . . . . . 150

6.3.4 Single-Cell Curved Box Girder Bridge . . . . . . . . . . . 152

6.3.5 Two-Cell Curved Box Girder . . . . . . . . . . . . . . . 152

6.3.6 Multi-Branch Box Girder Assemblage . . . . . . . . . . . 157

6.4 Prestressing, Temperature, Creep and Shrinkage Efferts $\quad . \quad . \quad . \quad 162$

6.4.1 Prestressing of Bax Gi-der Bridges . . . . . . . . . . . . . 162

6.4.2 Thermal Stress Analysis for Box Girders . . . . . . . . . . 165

6.4.3Shrinkage Analysis for Composite Box Girder Bridge . . . . 168

6.4.4 Creep Analysis for Concrete Box Girder Bridge . . . . . . . 168

7 SUMMARY, CONCLUSIONS AND RECOMMENDATIONS · 173

7.1 Summary . . . . . . . . . . . . . . . . . . 173 
7.2 Conclusions . . . . . . . . . . . . . . . . . . . 174

7.3 Recommendations for Future Work . . . . . . . . . . . . . . . 175

REFERENCES . . . . . . . . . . . . . . . . . 176

APPENDIX A: COMPUTER PROGRAMS

AND USER MANUAL . . . . . . . . . . . . . . . . . . . . . 184 


\section{List of Figures}

2.1 Thin-Walled Beam Subjected to Bending Moments $M_{x}$ and $M_{y}$. . . . . 10

2.2 Flexural Shear Flow in Box Beam . . . . . . . . . . . . . . . . . . 11

2.3 Statically Indeterminate Shear Flows in Multicell Box Bean . . . . . . . 13

2.4 Actual Bending Stress Distribution in a Thin-Walled Beam . . . . . . . 13

2.5 Biaxially Symmetric Single-Cell Box Beam for Shear Lag Analysis . . . . It

2.6 Reissener's Hypothesis for Shear Lag Analysis . . . . . . . . . . . . . 15

2.7 Basic Warping Displacements for Multicell Box Beam Shear Lag Anajysis . I8

2.8 Single-Cell Box Beam Subjected to Pure Torsion $T_{\text {. }}$. . . . . . . . . . . 20

2.9 Shear Flows Due to Pure Torsion in Multicell Box Beam . . . . . . . . 21

2.10 Definition of Sectorial Coordinate $\omega(s)$. . . . . . . . . . . . . . . . . . . . 24

2.11 Thin-Walled Box Beam for Torsional Warping Analysis . . . . . . . . . 26

2.12 Single-Cell Box Girder Subjected to Eccentric Loading . . . . . . . . . 28

2.13 Definition of Distortional Angle $\beta$. . . . . . . . . . . . . . . . . . . . . . . . 29

2.14 Definition of Stress Resultants and Displacements . . . . . . . . . . . . 29

2.15 Beam-Column on Elastic Foundation Analogy for Distortional Analysis . 32

2.16 Types of Intermediate Diaphragms _. . . . . . . . . . . . . . . . . . . 33

2.17 Basic Distortional Modes for Various Cross sections _ . . . . . . . . . . . 35

2.18 Transverse Bending Due to Different distortional Modes . . . . . . . . . $3 k$

2.19 Transverse Bending Due to Loads Applied Between Webs . . . . . . . . . 38

3.1 Beam-Column Analogy for Torsional Warping and Shear Lag . . . . . . 50

3.2 Beam-Column-on-Elastic-Foundation Analogy for Distortion . . . . . . . 52

3.3 Geometry of Multicell Box Beam Element . . . . . . . . . . . . . . 57

3.4 Distributed Load $q_{1}(z)$. . . . . . . . . . . . . . . . . . . . . . . 59

3.5 Distributed Moment $m_{i}(z)$. . . . . . . . . . . . . . . . . . . . . 60

3.6 Concetrated Point Load $P_{0}$. . . . . . . . . . . . . . . . . . . . . . . . . . . . 6()

3.7 Concentrated Moment $M_{0}$. . . . . . . . . . . . . . . . . . . . . 61

3.8 Beam Subjected to Point Load $P_{0}$ and Idealized as Two Elements . . . . 69

4.1 Coordinate System for Curved Box Beam . . . . . . . . . . . . . . . . 74

4.2 Loads, Forces and Displacements of a Curved Beam . . . . . . . . . . . 75

4.3 Rotation Due to Uniform Axial Displacement $w$. . . . . . . . . . . . 76

4.4 Definition of Angle of Twist $\quad$. . . . . . . . . . . . . . . . . . . . . . . . . . . . 77

4.5 Biaxially Symmetric Single-Cell Curved Box Beam for Shear Lag Aralysis . 79

4.6 Stress Resultants and Displacements for Distortional Analysis . . . . . . 82

4.7 Coordinates for Non-Prismatic Curved Box Beam . . . . . . . . . . . . . 85

4.8 Displacements at Midsurface of the Cross Section . . . . . . . . . . . . . . 86

4.9 (a) Transverse Displacement Functions $\Psi(s)$. . . . . . . . . . . . . . . . . . 89

4.9 (b) Transverse Displacement Functions $\equiv(s)$. . . . . . . . . . . . . . . . . 90 
4.10 Geometry of Curved Multicell Box Beam Element . . . . . . . . . . . 92

4.11 Geometry of Mapping Element . . . . . . . . . . . . . . . . . . . 93

4.12 Box Girder Assemblage . . . . . . . . . . . . . . . . . . . . . . . 98

4.13 Intersection of Box Girder Bridge Assemblage . . . . . . . . . . . . . . . . . . 99

5.1 Prestressed Bar Element . . . . . . . . . . . . . . . . . . . . . 106

5.2 Bridge Cross Sections Analysed by Elbadry and Ghali . . . . . . . . 109

5.3 Temperature and Stress Distributions . . . . . . . . . . . . . . . 110

5.4 Temperature and Stress Distributions . . . . . . . . . . . . . . . 111

5.5 Temperature Distribution from Priestly . . . . . . . . . . . . . . 112

5.6 Creep of Concrete Under the Effect of Sustained Stress . . . . . . . . 115

5.7 Stress Variation with Time $t$. . . . . . . . . . . . . . . . . . . 118

5.8 Phenomenon of Relaxation . . . . . . . . . . . . . . . . . . . 119

5.9 Step-by-Siep Numerical Integration . . . . . . . . . . . . . . . . 121

6.1 Single-Cell Single Span Box Girder . . . . . . . . . . . . . . . . 127

6.2 Vertical Deflection . . . . . . . . . . . . . . . . . . . . . . . 128

6.3 Longitudinal Normal Stress Along Junction A . . . . . . . . . . . . 128

6.4 Longitudinal Stress Distributions for Single-Cell Box Girder . . . . . . . 129

6.5 Transverse Stress Distributions for Single-Cell Box Girder . . . . . . . 130

6.6 Two-Cell Two Span Box Girder . . . . . . . . . . . . . . . . . . 132

6.7 Vertical Deflection . . . . . . . . . . . . . . . . . . . . . . . 133

6.8 Longitudinal Normal Stress Along Junction A . . . . . . . . . . . . 133

S.9 Longitudinal Stress Distributions for Two-Cell Box Girder . . . . . . . 134

6.10 Transverse Stress Distributions for Two-Cell Box Girder . . . . . . . 135

6.11 Three-Cell Single Span Box Girder . . . . . . . . . . . . . . . . 137

6.12 Finite Element Mesh for Grillage Analysis . . . . . . . . . . . . . 138

6.13 Vertical Deflection . . . . . . . . . . . . . . . . . . . . . . 138

6.14 Longitudinal Stress Distribution at Midspan _. . . . . . . . . . . . 139

6.15 Transverse Stress Distributions for Three-Cell Box Girder . . . . . . . 140

6.16 Two-Spine Single Span Box Girder . . . . . . . . . . . . . . . . 142

6.17 Deflection at Midspan . . . . . . . . . . . . . . . . . . . . . . 143

6.18 Longitudinal Normal Stress Distribution at Midspan . . . . . . . . . 143

6.19 Cross Section of a Straight Single-Cell Box Girder . . . . . . . . . . 144

6.20 Comparison with Shell Finite Element Method . . . . . . . . . . . 145

6.21 First Experimental Model of Kristek . . . . . . . . . . . . . . . . 147

6.22 Second Experimental Model of Kristek . . . . . . . . . . . . . . . 148

6.23 Comparison of Longitudinal Warping Stresses Along Span . . . . . . . 148

6.24 Deffection Curve in Second Step of Kristek's Analysis . . . . . . . . . 149

6.25 Two-Cell Skewed Box Girder Bridge . . . . . . . . . . . . . . . . 151

6.26 Single-Cell Curved Box Girder . . . . . . . . . . . . . . . . . . 153

6.27 Comparison with Macro Thick Shell Finite Ciement Method . . . . . . 154

6.28 Two-Cell Curved Box Girder . . . . . . . . . . . . . . . . . . . 155 
6.29 Comparison with Spline Finite Strip Method . . . . . . . . . . . . 156

6.30 Multi-Branch Box Girder Assemblage . . . . . . . . . . . . . . . 158

6.31 Variation of Longitudinal Stress Along Junction A for Branch 1 $\quad$ - . . 159

6.32 Variation of Longitudinal Stress Along Junction A for Branch 3 . . . . 160

6.33 Longitudinal Normal Stress Along Cross Sections . . . . . . . . . . 161

6.34 Prestressed Box Girder . . . . . . . . . . . . . . . . . . . . . 163

6.35 Longitudinal Stress at Midspan due to Prestressed Forces . . . . . . . 16.4

6.36 Prestressing Camber . . . . . . . . . . . . . . . . . . . . . . 164

6.37 Box Girders for Thermal Stress Analysis . . . . . . . . . . . . . . 166

6.38 Temperature Distribution . . . . . . . . . . . . . . . . . . . . 166

6.39 Longitudiral Thermal Stress Distributions . . . . . . . . . . . . . 167

6.40 Two-Cell Two Span Composite Box Girder . . . . . . . . . . . . . 169

6.41 Longitudinal Normal Stress due to Shrinkage . . . . . . . . . . . . 170

6.42 Longitudinal Normal Stress Distributions due to Creep . . . . . . . . 171

A.1 Node and Wall Definition of Cross Section . . . . . . . . . . . . . 192

A.2 Loading Information for Curved Element . . . . . . . . . . . . . . 193

A.3 Prestressed Bar Information . . . . . . . . . . . . . . . . . . . 194

A.4 Definition of Types of Temperature Distribution . . . . . . . . . . . . . . 19.4 


\section{LIST OF IMPORTANT NOTATIONS}
A
[B]
$=$ cross-sectional area
$[C],\left[C_{a}\right],[\bar{C}]$
= strain-displacement matrix
= basic, partially orthogonalized and fully orthogonalized matrices of properties in warping
$\{d\}$
$\{D\}$
$\{\tilde{D}\}$
$\{\dot{D}\}^{e}$
$E$
$[E]$
$\{F\}^{e}$
$\left\{F_{p s}\right\}$
$\left\{F_{p}\right\}$
$\left\{F_{s h}\right\}$
$G$
$\stackrel{h}{[H]},\left[H_{a}\right],[\bar{H}]$
$=$ displacement vector for the homogeneous solution of the governing differential equation
$=$ displacement fields
= displacement fields corresponding to the orthogonalized coordinates
$=$ nodal displacement vector corresponding to the orthogonalized coordinates
$=$ Young's modulus of elasticity
$=$ elastic matrix
$=$ equivalent element nodal force vector due external loads
$=$ equivalent element nodal force vector due to prestressing force
$=$ equivalent element nodal force vector due to temperature
$=$ equivalent element nodal force vector due to shrinkage and creep
$=$ shear modulus
$=$ wall thickness of cross-section
= basic, partially orthogonalized and fully orthogonalized matrices of section properties in distortion
$I_{x}, I_{y}$
$=$ moments of inertia about $x, y$ axes, respectively
$I_{\omega}$
$I_{d t}$
$=$ sectorial moment of inertia
$=$ distortional moment of inertia
$I_{n !}$
$J_{d_{1}}$
= shear lag warping moment of inertia
$=$ distortional shear constant
$J_{v}$
$J^{\prime \prime}$
$=$ St. Venant's torsional constant
$=$ shear lag constant
$[J],\left[J_{a}\right],[\bar{J}]$
= basic, partially orthogonalized and fully orthogonalized matrices of section properties in torsion

$k$
$l$
$m$
$m_{1}$
$\bar{m}$
$\left\{M_{s}\right\}$
$M_{x}, M_{y}$
$M_{\infty}$
$n$
$n$
$\dot{n}$
$=$ number of concentrated loads
$=$ length of box beam element
$=$ number of shear lag modes
$=$ torsional moment per unit length
$=4+n+m$
$=$ transverse bending moment due to unit distortion
$=$ bending moment in $x$ and $y$ directions
$=$ bimoment due to warping
$=$ number of distortimnal modes
$=4+n$
$=$ axial force of $L$ in:-:olumn 


$$
\begin{aligned}
& \text { [N] } \quad=\text { shape function matrix } \\
& P_{x}, P_{y}, P_{z} \quad=\text { conentrated loads in } x, y, z \text { directions } \\
& P_{0} \quad=\text { prestressed force } \\
& q_{b}=\text { shear flow due to bending } \\
& q_{s}, q_{\omega}=\text { shear flow due to pure torsion and torsional warping } \\
& q_{d}, q_{d \omega} \quad=\text { shear flow due to pure distortion and distortional warping } \\
& q_{x}, q_{y}, q_{z} \quad=\text { distributed loads per unit length in } z, y_{y} z \text { directions } \\
& q_{d i}, q_{v i} \\
& Q_{x}, Q_{y} \\
& \{r\} \\
& \left\{r_{x}^{*}\right\},\left\{r_{y}^{*}\right\} \\
& R_{0} \\
& \boldsymbol{s} \\
& {\left[S_{p s}\right]^{e}} \\
& {[S]^{e}} \\
& T(x, y) \\
& T_{s}, T_{\omega} \\
& T_{d}, T_{d \omega} \\
& T_{z} \\
& {\left[T_{a}\right],\left[T_{b}\right],\left[T_{c}\right]=\text { transformation matrices for two step orthogonalization }} \\
& {[T] \quad=\left[T_{c}\right]\left[T_{b}\right]\left[T_{a}\right]} \\
& u, v, u \\
& v_{n}, v_{s} \\
& x, y, z \\
& \hat{u} \\
& \alpha \\
& \alpha_{3}, \alpha_{z} \\
& \beta_{1} \\
& \bar{\beta}_{i} \\
& \chi \\
& x\left(t, t_{0}\right) \\
& \gamma_{s z} \\
& \delta \\
& \delta W_{e} \\
& \delta W_{1} \\
& \epsilon_{c s} \\
& \left(\epsilon_{c s}\right)_{u} \\
& \epsilon_{z} \\
& \theta_{2} \\
& \lambda \\
& \rho \\
& =\text { generalized force dual of distortional (shear lag) warping function } \\
& =\text { shear forces in } x \text { and } y \text { directions } \\
& =\text { vector of angles and distances in distortional and shear lag } \\
& =\text { vectors describing the position of the loads on the cross-section } \\
& =\text { radius of curved box beam } \\
& =\text { perimeter coordinate along the middle line of box walls } \\
& =\text { element stiffness matrix for prestressing bar element } \\
& =\text { element stiffness matrix } \\
& =\text { temperature distribution for box beam } \\
& =\text { torsional moments due to pure torsion and torsional warping } \\
& =\text { torsional moments due to pure distortion and distortional } \\
& \text { warping } \\
& =\text { torsional moment } \\
& =\text { dispacement components in } x, y, z \text { directions } \\
& =\text { dispacement components in the normal and tangent directions of } \\
& \text { the walls of box beam } \\
& =\text { Cartesian coordinate axes } \\
& =\int w d z \\
& =\text { the angle between } z \text { axis and the tangent at point }(x, y) \text { on the } \\
& \text { wall of the cross-section } \\
& =\text { coefficients of thermal expansion in the } s \text { and } z \text { directions } \\
& =\text { distortional mode } \\
& =\text { distortional mode in orthogonalized coordinate system } \\
& =1 / R_{0} \\
& =\text { aging coefficient } \\
& =\text { shear strain } \\
& \text { = variational symbol denoting virtual quantities } \\
& \text { = external virtual work } \\
& =\text { internal virtual work } \\
& =\text { free shrinkage strain } \\
& \text { = ultimate free shrinkage strain } \\
& =\text { longitudinal normal strain } \\
& =\text { angle of twist } \\
& =\text { eigenvalue } \\
& =\text { perpendicular distance from shear center to point }(x, y) \text { on the }
\end{aligned}
$$




$$
\begin{array}{ll}
\sigma_{z} & =\text { longitudinal normal stress } \\
\tau_{\Delta z} & =\text { shear stress } \\
\bar{\tau} & =\text { shear stress distribution function } \\
\psi_{\mathbf{v}} & =\text { shear lag mode } \\
\bar{\psi}_{\mathbf{v}} & =\text { shear lag mode in orthogonalized coordinate system } \\
\{\Psi(s)\},\{\Xi(s)\} & =\text { displacement shape functions along the walls of a box beam } \\
\omega & =\text { torsional warping function } \\
\omega_{d,}, \omega_{v t} & =\text { distortional and shear lag warping functions, respectively } \\
\{\omega\} & =\text { warping function vector } \\
\{\bar{\omega}\} & =\text { warping function vector corresponding to the orthogonalized } \\
\varphi\left(t, t_{0}\right) & =\text { coordinates } \\
\varphi_{u} & =\text { ultimate creep coefficient } \\
\xi, \eta, \zeta & =\text { geometry mapping coordinates }
\end{array}
$$




\section{INTRODUCTION}

\subsection{Statement of the Problem}

Box girder structures are widely used in modern medium and long span bridges. In addition to economic and aesthetic considerations, box girders are used mainly for their high torsional rigidity. Maisel (1974) conducted a detailed survey of the box girder bridges built world-wide until 1972. Since that time many more bridges of this kind have been constructed and, as stated by Schlaich and Scheef (1981), today the box cross-section is usually considered as a design alternative for medium and long span bridges.

Despite the preponderance of such bridges, codes of practice, such as BS5400 (1979), AASHTO (1989) and OHBDC (1983), do not provide detailed provisions for their analysis and design. This is particularly the case for multicell and curved box girders. In most cases the codes recommend that the loading effects which cannot be predicted by the elementary or engineer's beam theory, such as shear lag effects, warping stresses due to torsion and distortion, transverse stresses due to distortion, effects of prestressing, temperature, creep and shrinkage, should be considered and theoretically sound principles should be applied in their determination. In practice, several methods with various degrees of rigour are available for analysis. These range from the elementary or engineer's beam theory to complex shell finite element analyses; other methods of analysis utilize iolded plate (Chu and Pinjarkar 1971, Scordelis 1974) and finite strip (Cheung 1976, Loo and Cusens 1978) methods. While some of the latter methods are general and accurate, their use in early stages of design may not be warranted. This is especially true about the finite element method which 
requires a great amount of effort and tends to produce excessive amounts of output whose interpretation can be time consuming. The more sophisticated methods also obscure the significance of the contribution of individual structural actions to the over-all resistance of the box girder. For example, it is not easy in a finite element analysis to isolate the effect of torsional and distortional warping on normal stresses from that of extension and bending. This also applies to folded plate and finite strip analyses.

Due to those problems, researchers and designers have been using an advanced form of Vlasov's thin-walled beam theory (Roik and Sedlacek 1970, Sedlacek 1968) to analyze box girders. An extensive survey of the applications of this theory has heen provided by Maisel (1982). The accuracy of the stresses calculated by the more advanced version of the theory is adequate for practical applications. Furthermore, the method clearly and elegantly accounts for each type of structural action and for its relative significance in the overall behaviour of the box. It is also efficient and, once programmed on the computer, requires mimimal input data and computer time.

To date, however, existing applications have been limited to straight right box girders, and temperature and time dependent effects, such as prestressing, creep and shrinkage, have not been integrated into this theory. The extended version of Vlasov's thin-walled beam theory is not applicable to curved, haunched and skew box girders in its existing form; modifications are needed for its extension to the latter situations.

\subsection{Previous Work}

During the past several decades, extensive experimental and theoretical investigations on box girder structures have been carried out. An excellent state of the art review was given by Maisel (1970), while more recent surveys can be found in $\mathrm{Li}(1987)$ and Nakai and Yoo (1988). No attempt will be made here to repeat the work in these surveys. Only those studies that are of direct relevance to the subject 
of this thesis will be reviewed.

The warping phenomenon of thin-walled beam cross section due to twisting. which produces additional normal stresses in the cross section, was first discovered by Timoshenko (1905) in his early work on the instability of I beams. Vlasov (1961) systematically extended the engineer's beam theory by introducing the concept of sectorial coordinate and torsional warping in thin-walled beam structures, which led to the so-called Vlasov's thin-walled beam theory. A general method for deformable closed cross sections was developed based on generalized coordinates and the principle of virtual work. Since Vlasov's early work, a number of researchers have cortributed to this theory, as pointed out by Maisel (1970). Benscoter (1954) and Dabrowski $(1965,1968)$ reformulated Vlasov's theory for multicell and curved box beams, while Heins and Oleinik (1976) gave a finite difference solution of Vlasov's equations for curved box girders.

Kollbrunner and Hajdin $(1966,1975)$ treated thin-walled beam structures similar to Vlasov but in a more general form by including shear deformations for closed thinwalled cross sections. The formulation of torsional warping in the present thesis is based on the theoretical work of the latter investigators. Waldron (1988) conducted a parametic study for concrete straight single-cell box girders to investigate the significance of torsional warping. He concluded that for a typical single-cell box girder, torsional warping stresses could be up to $29 \%$ of the longitudinal normal stresses around the midspan section. For the fully fixed single span girder this figure could be double the above amount.

Dabrowski (1968) introduced distortional warping and presented the fundmental equations for deformable cross section curved box girders. Li (1987), and Nakai and Yoo (1988) used those equations to study the distortional behaviour of concrete and steel single-cell box girders. Distortion of prestressed concrete box girders was considered by Hajdin and Dunica (1974). Kristek (1970) used folded plate: theory to study haunched deformable cross section box girders.

A general analytical model for distortional analysis of single-cell and multicell straight box girders was proposed by Sedlacek (1968). For multicell boxes, more than 
one distortional mode was introduced in order to describe the complete distortional behaviour of the cross section. Warping functions were defined for each mode of distortion. A two step orthogonalization procedure was used to uncouple structural action modes. The governing differential equations corresponding to the uncoupled structural actions were similar to those of beam-column on elastic foundations.

For shear lag analysis, extensive investigations for steel and composite box girders huve been undertaken by Moffatt and Dowling $(1975,1978,1978)$ using traditional finite element methods. Based on these investigations, they developed approximate expressions which can be used in practical design.

An analytical method for shear lag analysis in single-cell box beams was given by Reissner (1938). A longitudinal deformation function was introduced to describe the warping behaviour due to shear deformations in a wide flange box beam and the governing differential equations were derived from the principle of minimum potential energy. Foutch and Chang (1982), and Chang and Fang (1987) investigated the negative shear lag effects for a cantilever box beam in a similar way as Reissner. Chang and Ding (1989) studied shear lag effects in box girder with varying depth. Nakai and Yoo (1988) investigated shear lag effects for $\pi$ girders. Dezi and Mentrasti (1984) introduced three longitudinal deformation functions for trapezoidal single cell box girders with overhangs. These studies clearly show that shear lag deformations can introduce normal stresses which are at least $30 \%$ higher than those predicted by elementary beam theory.

Roik and Sedlacek $(1966,1970)$ studied shear lag effects for single and multicell box girders and treated the problem mathematically similarly to the distortional analysis of Sedlacek. They defined warping functions for each shear lag mode and developed the governing differential equations which have the same basic form as that for torsional warping given by Vlasov's theory.

Maisel (1982) developed an analytical procedure for distortional and shear lag analysis of single and multicell box girders based on the work of Heilig, Kollbrunner and Hajdin, Sedlacek and Roik, and Sedlacek. Some practical numericai examples were solved using hand calculation a:d the force method of analysis. A computer 
program based on Maisel's proceedure was developed by Mavaddat and Mirza (1989).

The methods and procedures described thus far lack generality or can be very tedious to use. The finite element method is recognized as the most powerful and versatile numerical technique for engineering analysis. During the past two decades, many finite element models have been developed for box girder analysis. Among them, a model for arbitrary geometry box girders was developed by Sisodiya and Ghali (1973), Fam and Turkstra (1975) developed some special elements for curved box girder bridges. Moffatt and Lim (1976) used triangular and trapezoidal plane elements to analyze straight and curved box girder structures while Jirousek and Bouberguig (1979) presented a macro-element based on modified Ahmad's thick shell element for prestressed curved box girder analysis. Flat thin shell elements were also developed by Lyon (1977) and Razaquur and Nofal (1989) for modelling the box girder as an assemblage of thin facet shells. While these elements are quite general, they need extensive computer resources and much effort on the part of the designer. They are time-consuming and need extensive data input and produce massive output, which sometimes masks the physical understanding of box girder behaviour. A logical alternative for modelling medium and long span box girder bridges is to develop thinwalled box beam finite elements which are similar to one dimensional beam elements. The latter has been the objective of a number of investigations over the last three decades.

Thin-walled beam finite element models have been developed by several investigators based on Vlasov's theory (Krajcinovic 1969; Yoo 1980; Gunnlaugsson and Pedersen 1982 and Waldron 1986). However, all of these models disregard crosssectional distortion and shear lag effects. Based on the generalized Vlasov's thinwalled beam theory, Bazant and El Nimeiri (1974) developed a skew-ended thinwalled box beam finite element for curved and straight single cell box girders in which transverse distortion and longitudinal torsional warping were included, but their model did not take into account shear lag. A similar approach was adopted by Mikkola and Paavola (1980), while Zhang and Lyons (1984) developed a thin-walled box beam finite element which is applicable to the analysis of single spine multicell box girder structures. For multi-spine boxes, two dimensional grillage subdivision 
was used to idealize the structures. Only one distortional mode was considered and the effective width concept was used to approximate shear lag effects. The element employed conventional polynomial shape functions and thus each span of a box girder bridge had to be divided into several elements to obtain accurate results. Choudhury (1986) combined the Bazant - El Nimeiri, and the Zhang - Lyons models to develop a curved nonprismatic thin-walled single-cell box beam element tor nonlinear analysis of reinforced and prestressed concrete box girder bridges.

For temperature effects, Churchward and Sokal (1981) and Elbadry and Ghali (1983) studied the distribution of temperature in concrete bridges. Temperature stresses in concrete and composite box girder bridges were investigated by Prakash (1986), Waldron and Ramezankhani (1990) and Dilger et al. (1983\%. Chan et al. (1990) measured the temperature variation in three composite box girder bridges in North America and analyzed the corresponding thermal stresses by facet shell finite elements. Thermal stresses in a skewed bridge deck was considered by Moorty and Roeder (1990). In determining thermal stresses, with the exception of Waldron and Ramezankhani, the other investigators mentioned above used standard shell finite elements.

Argyris et al. (1977) proposed a general constitutive model for concrete creep and shrinkage employing finite element analysis. Composite box girder bridge and curved box girder bridge behaviour under construction stages was studied by Branco and Green (1985), and Tadros, Ghali and Dilger (1982). Kristek and Bazant (1987) studied shear lag effect in concrete box girders using a probability creep model. Prestress losses due to the effects of time dependent concrete properties were considered by Shiu and Russell (1987). Prasada and Jayaraman (1989) investigated creep and shrinkage effects in partially prestressed concrete members. A general procedure to calculate temperature stresses, creep and shrinkage effects for concrete, prestressed concrete and composite members was given by Ghali and Favre (1986). However, the application of this procedure to specific structures requires proper adaptations and modifications.

It is : nportant to mention that extensive analytical models for linear and non- 
linear analysis of reinforced and prestressed concrete box girder bridges have been developed at the University of California at Berkeley during the past two decades. Scordelis (1982) has reviewed these contributions. The analytical methods and com. puter programs that he has discussed provide the capability for the detailed analysis of general single-cell and multicell box girder bridges based mostly on conventional finite element procedures, or folded plate theory.

In summary, although extensive work has been carried out to study the static response and temperature and prestressing effects in box girder bridges, no work has been carried out to integrate a general thin-walled beam theory with prestressing and thermal analysis of box girders. Since prestressing in curved box girders can produce torsion, one must investigate the effect of these torsional forces on warping and distortion of the cross section. A similar situation may exist due to variable temperature distribution in a box girder.

\subsection{Objectives and Scope}

Based on the preceding review, the main objectives of this thesis are:

1. To develop general thin-walled box beam finite elements for the static analysis of multicell box girder bridges;

2. To develop a method of analysis for the prestressing, creep and shrinkage analysis of multicell thin-walled box girders;

3. To develop a method of analysis for the thermal stress analysis of multicell box girders;

4. To develop working programs that implement the above methodologies and which can be used to analyze multicell concrete and steel-concrete composite box girder bridges for the effects of gravity loads, prestressing and temperature;

5. To verify those computer programs by comparing their predicted results with those obtained from siseil finite element analyses, other refined methods of anal. 
ysis, or experimental data;

6. To demonstrate the efficiency and expediency of the proposed methods of analysis.

The proposed models will account rigorously for extension, bending, torsion, torsional warping, cross-sectional distortion, distortional warping and shear lag effects. The effects of preatressing, temperature, creep and shrinkage will also be included. For the straight right box girders, the uncoupled governing differential equations of extension, flexure, torsion, distortion and shear lag will be solved exactly to develop the appropriate shape functions. This exact shape function finite element model will eliminate the need for dividing the bridge into many elements in each span. For curved box girders, it is not simple to find the general solution of the governing differential equations, thus, conventional polynomial shape functions will be used. The second model will be mainly for the analysis of curved, haunched and skew multicell box girders.

One of the advantages of the proposed method is that the different structural actions can be uncoupled. This permits the analyst to study each action separately, resulting in a better insight into the behavior of the structure. The computer resource requirements of the proposed elements would be small and thus they can be easily implemented on practically any microcomputer. The speed of analysis and the transparent output will permit a designer to study the various design options accurately and rapidly, saving time and resources.

The theory presented in this thesis will be based on linear elasticity and will be limited to beam-type thin-walled structures. For single-spine box girders, the theory is apflicable to boxes comprising up to four cells. Five or more cell boxes are likely to behave as a cellular plate rather than a beam. For multi-spine box girders, the limitation is a maximum of two spines. 


\section{BASIC THEORY OF THIN-WALLED BOX BEAMS}

\subsection{General}

This chapter will present the basic theory of thin-walled box beams which constitutes the theoretical basis of this thesis. The chapter consists of four important and fundamental sections dealing with thin-walled box beam behaviour: flexure, torsion, distortion and shear lag. For clarity, the discussion will be restricted to prismatic right straight thin-walled box beams, but the extensions to non-prismatic and curved beams will be evider:. Linear elasticity and small displacements are assumed throughout the thesis.

\subsection{Longitudinal Bending}

\section{Normal stress}

For an asymmetric cross section, as shown in Fig. 2.1, where bending moments $M_{x}$ and $M_{y}$ are applied with respect to the non-principal horizontal and vertical axes $(x, y)$ through the centroid $C$, respectively, the flexural longitudinal normal stress $\sigma_{z}=\sigma_{b}$ at any point $(x, y)$ is given by the conventional Bernoulli-Euler's bean theory (Oden and Ripperger 1981) as

$$
\sigma_{b}=\frac{M_{y} I_{z}-M_{x} I_{x y}}{I_{x} I_{y}-I_{z y}^{2}}+\frac{M_{z} I_{y}-M_{y} I_{2 y}}{I_{z} I_{y}-I_{z y}^{2}} y
$$


where the geometric moments of inertis are given by

$$
\begin{aligned}
& I_{z}=\int_{A} y^{2} d A \\
& I_{y}=\int_{A} x^{2} d A \\
& I_{x y}=\int_{A} x y d A
\end{aligned}
$$

$A$ is the cross sectional area of the beam.

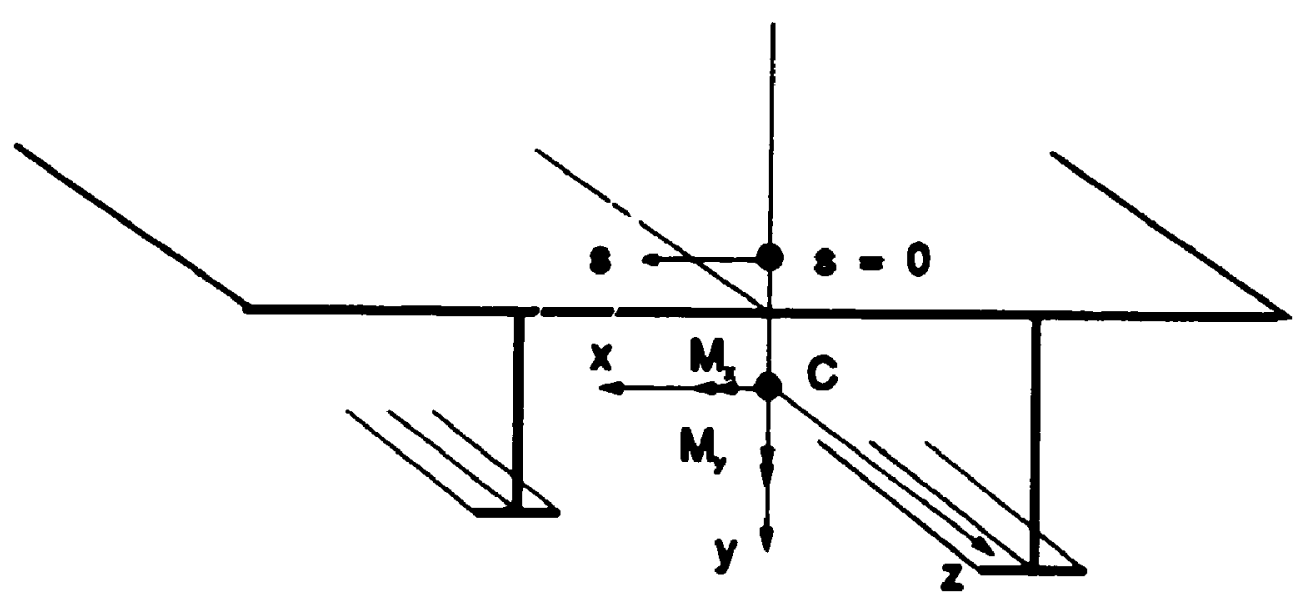

Fig. 2.1 Thin-walled beam subjected to bending moments $M_{x}$ and $M_{y}$. Shear streas

For thin-walled open cross section beams, the flexural shear stress $\tau_{s z}=\tau_{b}$ is given by

$$
q_{b}=-\frac{Q_{y} I_{y}-Q_{x} I_{x y}}{I_{x} I_{y}-I_{x y}^{2}} S_{x}-\frac{Q_{x} I_{x}-Q_{y} I_{x y}}{I_{x} I_{y}-I_{x y}^{2}} S_{y}
$$

where $q_{0}=r_{t} t$ is the shear fow due to longitudinal bending, $Q_{x}$ and $Q_{y}$ are the shear forces in the horizontal and vertical directions, respectively, while

$$
\begin{aligned}
& S_{x}=\int_{0}^{s} y t d s \\
& S_{y}=\int_{0}^{s} x t d s
\end{aligned}
$$

Here $t$ is the thickness of the beam walls and $s$ is the curvilinear coordinate taken along the perimeter of the cross section and is measured from the point of zero shear. 
For the case $Q_{x}=0$, Eq. (2.2.6) reduces to

$$
q_{b}=-\frac{I_{y} S_{x}-I_{x y} S_{y}}{I_{x} I_{y}-I_{x y}^{2}} Q_{y}
$$

Furthermore, if either $x$ or $y$ is an axis of symmetry, $I_{x y}=0$, and the sbove equation reverts to the following well-known formula

$$
q_{b}=-\frac{S_{x}}{I_{x}} Q_{y}
$$

For a thin-walled box beam, the calculation of flexural shear stress is a statically indeterminate problem. To obtain the solution, an imr.ginary cut is inserted at an arbitrary point of the cross section as shown in Fig. 2.2. The shear flow q60 for this open cross section is given by Eq. (2.2.6). Next a statically indeterminate shear flow, $q_{b i}$, which is constant over the cross section, is introduced. The actual shear flow $q_{b}$ is given by the superposition of these two shear flows

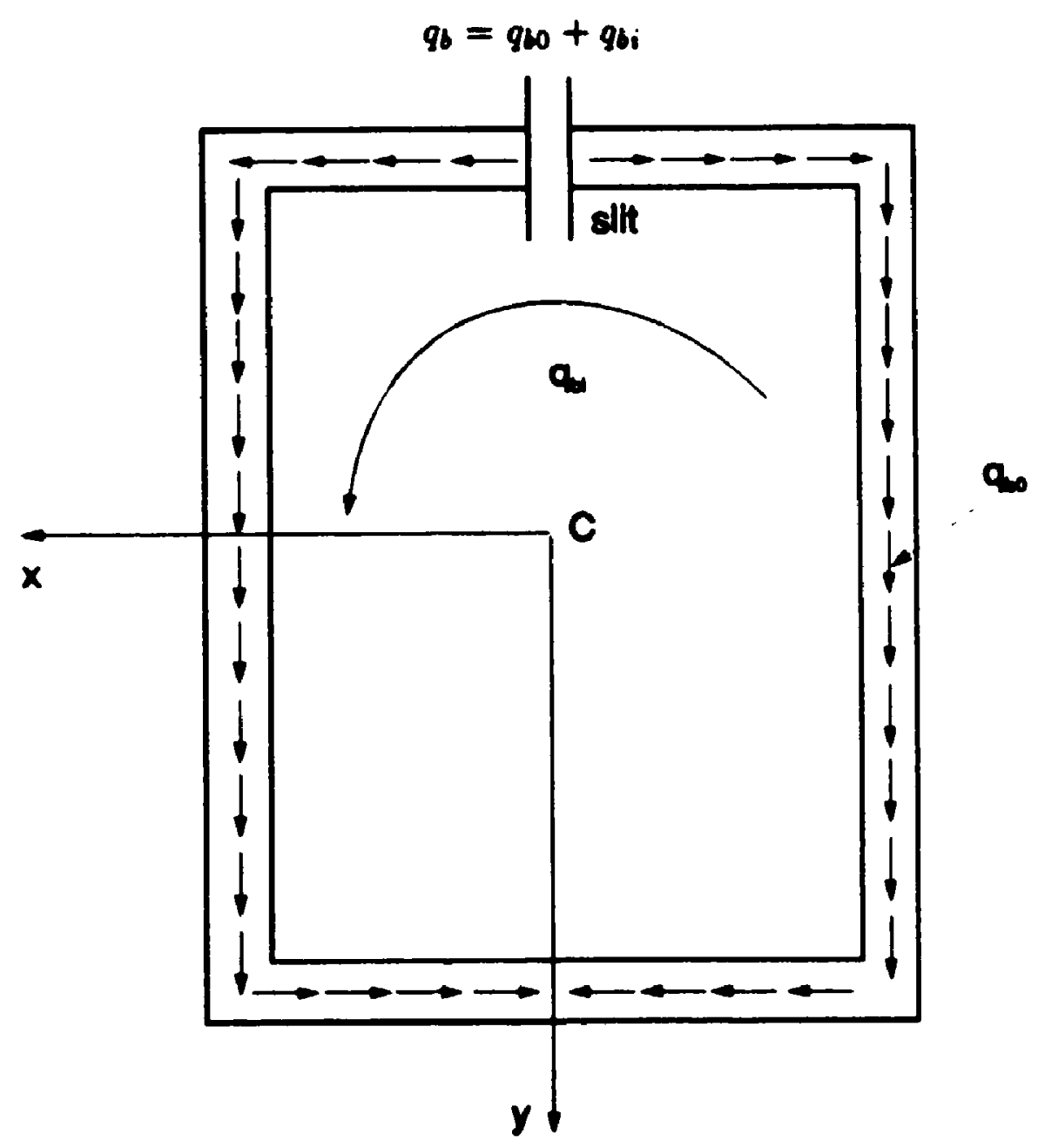

Fig. 2.2. Flexural shear flow in box beam. 
The statically indeterminate shear flow $q_{b}$ can be obtained by invoking the compatibility condition at the imaginary cut, i.e.

$$
\oint \gamma_{b} d s=0
$$

where $\gamma_{b}=\tau_{b} / G=q_{b} / G t$ is the shear deformation due to bending. Substituting Eq. (2.2.10) into Eq. (2.2.11), we have

$$
\oint \frac{q_{b 0}}{G t} d s+q_{b i} \oint \frac{d s}{G t}=0
$$

Thus $q_{b t}$ can be solved for as follows

$$
q_{b i}=-\frac{\oint \frac{q_{b 0}}{G t} d s}{\oint \frac{d s}{G t}}
$$

The same procedure can also be applied to multicell box beams as shown in Fig. 2.3. The imaginary cuts and the corresponding statically indeterminate s'.ear flows $q_{b 1}, q_{b 2}$ and $q_{b 3}$ are introduced for each cell, giving

$$
q_{b}=q_{b 0}+q_{b 1}+q_{b 2}+q_{b 3}
$$

and $q_{b 1}, q_{b 2}$ and $q_{b 3}$ can be obtained by solving the following simultaneous equations

$$
\begin{gathered}
\delta_{11} q_{b 1}+\delta_{12} q_{b 2}=\delta_{10} \\
\delta_{21} q_{b 1}+\delta_{22} q_{b 2}+\delta_{23} q_{b 3}=\delta_{20} \\
\delta_{31} q_{b 1}+\delta_{32} q_{b 2}+\delta_{33} q_{b 3}=\delta_{30}
\end{gathered}
$$

where

$$
\begin{aligned}
& \delta_{11}=\oint_{1} \frac{d s}{G t} \\
& \delta_{13}=\delta_{1 i}=-\int_{13} \frac{d s}{G t} \\
& \delta_{10}=-\oint_{1} \frac{960}{G t} d s
\end{aligned}
$$




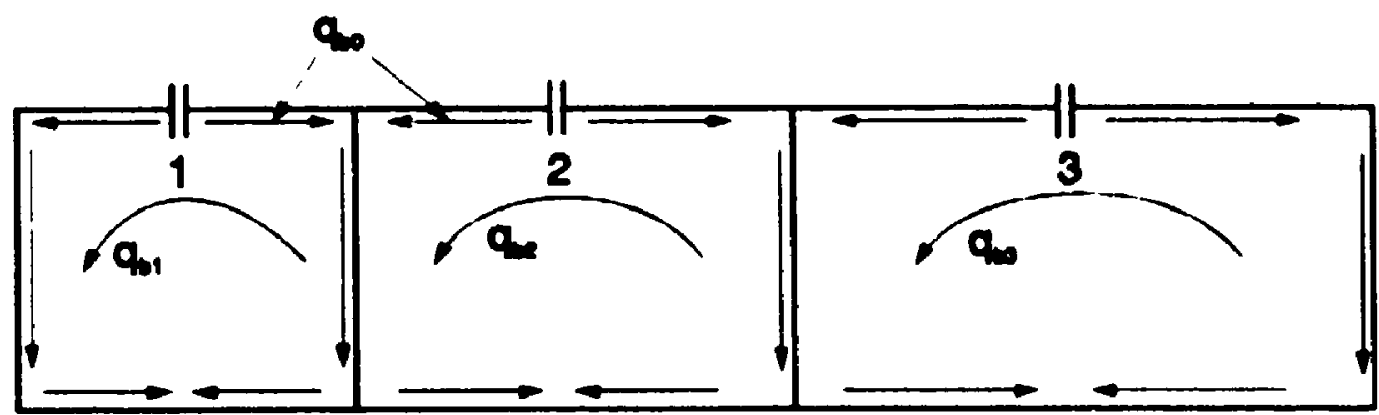

Fig. 2.3 Statically indeterminate shear flows in multicell box beam.

\subsection{Shear Lag}

Shear lag, or the non-uniform distribution of normal stress in the flanges of thinwalled beams, Fig. 2.4, is caused by lag of shear strains in the flange plates of thin-walled beams. Due to shear lag the maximum flexural normal stress usually occurs at the junction point of flanges and webs, and it may be significantly different. from the value obtained from coventional beam theory.

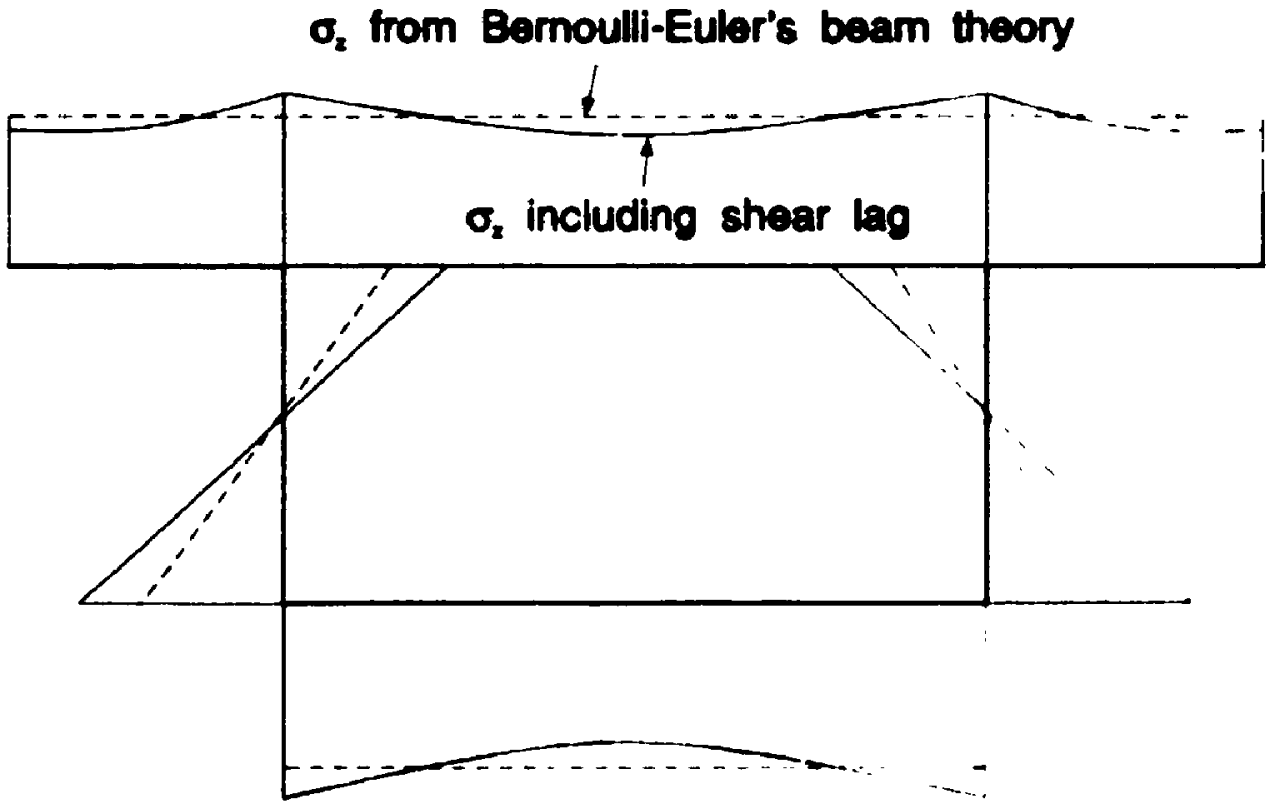

Fig. 2.4 Actual bending stress distribution in a thin-walled beam. 
The shear lag problem can be analyzed on the basis of Reissner's hypothesis (Reissner 1938), which states that the distribution of the flexural normal stresses in the flange plate of box beams can be appoximated by a second order parabolic curve. In order to illustrate the shear lag phenomenon in box beams, a biaxially symmetric single-cell box beam, as shown in Fig. 2.5, is considered first, and then the method will be extended to the more general multicell box beams.

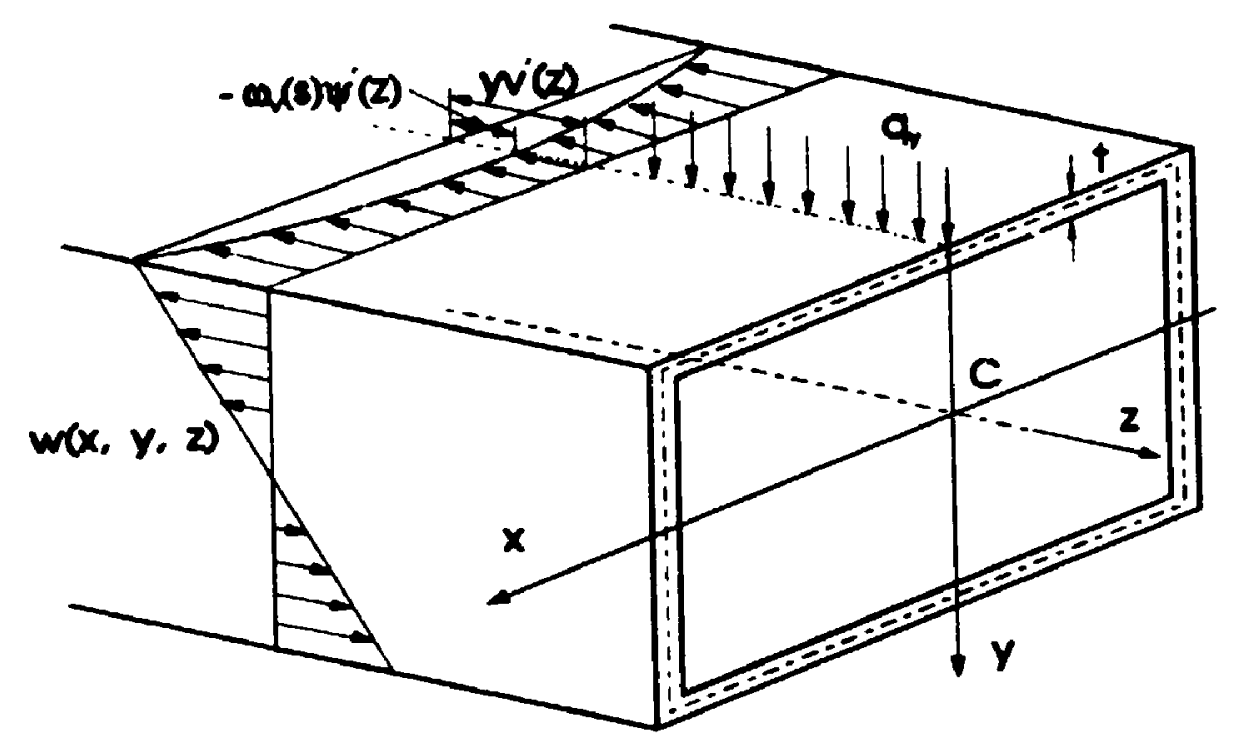

Fig. 2.5 Biaxially symmetric single-cell box beam for shear lag analysis.

For the single-cell bo: beam which is subjected to symmetric lateral load $q_{y}$ only, as shown in Fig. 2.5, by combining conventional beam theory and Reissner's hypothesis, the longitudinal displacement in the flange plates can be approximated as follows

$$
u(x, y, z)=-y v^{\prime}(z)-\omega_{v}(x, y) \psi^{\prime}(z)
$$

where $\omega_{v}(x, y)$ is the shear lag warping function based on Reissener's hypothesis as shown in Fig. 2.6, and $\psi(z)$ is a characteristic displacement function which represents the magnitude of the shear lag warping at any section along the beam. Note that $x$ and $y$ coordinates along the cross section can also be expressed, by means of a simple transformation, in terms of the curvilinear coordinate $s$ along the middle line 
box wall. Thus, Eq. (2.3.1) may be written alternatively as

$$
w(s, z)=-\omega_{b}(s) v^{\prime}(z)-\omega_{\prime \prime}(s) u^{\prime}(z)
$$

where $w(s, z)=w(x, y, z), \omega_{b}(s)=y$ and $\omega_{v}(s)=\omega_{v}(x, y)$.

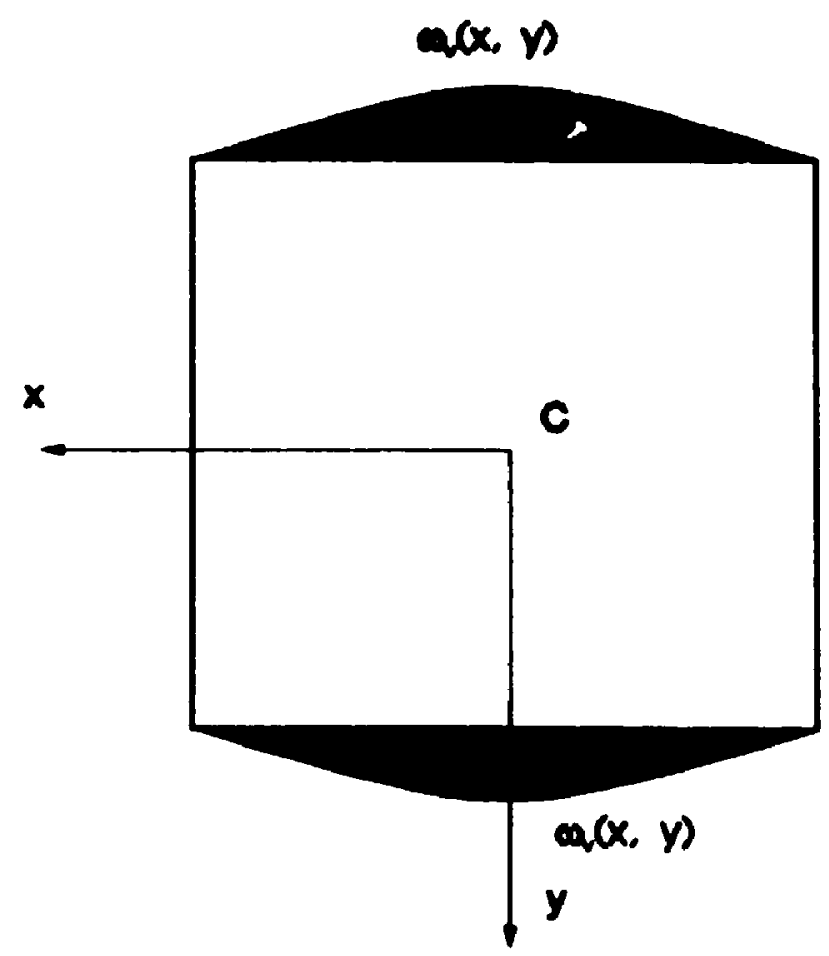

Fig. 2.6 Reissener's hypothesis for shear lag analysis.

From the theory of elasticity, and neglecting the contribution of transverse displacement, $v_{s}(s, z)$, to shear strain, the normal and shear strains are given, respectively, by

$$
\begin{aligned}
& \epsilon_{z}=\frac{\partial w(s, z)}{\partial z}=-\omega_{b}(s) v^{\prime \prime}(z)-\omega_{v}(s) \psi^{\prime \prime}(z) \\
& \gamma_{s z}=\frac{\partial w(s, z)}{\partial s}=-\dot{\omega}_{b}(s) v^{\prime}(z)-\dot{\omega}_{v}(s) \psi^{\prime}(z)
\end{aligned}
$$

where $\dot{\omega}_{b}(s)=d \omega_{b}(s) / d s$ and $\dot{\omega}_{v}(s)=d \omega_{v}(s) / d s$. Using Hooke's law, the normal stress, $\sigma_{z}$, and shear stress, $\tau_{s z}$, at any point $(s, z)$ are given by

$$
\begin{aligned}
& \sigma_{z}=E \epsilon_{z}=-E \omega_{b}(s) v^{\prime \prime}(z)-E \omega_{v}(s) \psi^{\prime \prime}(z) \\
& \tau_{s z}=G \gamma_{s z}=-G \dot{\omega}_{b}(s) v^{\prime}(z)-G \dot{\omega}_{v}(s) \psi^{\prime}(z)
\end{aligned}
$$

The governing differential equations of the problem can be developed by the principle of virtual work. The internal virtual work $\delta W_{1}$ equals

$$
\delta W_{1}=\int_{V}\left(\sigma_{z} \delta \epsilon_{2}+\tau_{s z} \delta \gamma_{s z}\right) d V
$$


where $V$ is the volume of the beam and the symbol $\delta$ denotes virtual quantities. Substituting Eqs.(2.3.3) and (2.3.4) into the above equation, yields

$$
\begin{aligned}
\delta W_{i}= & \int_{V} E\left(\omega_{b}^{2} v^{\prime \prime} \delta v^{\prime \prime}+\omega_{b} \omega_{v} v^{\prime \prime} \delta \psi^{\prime \prime}+\omega_{b} \omega_{v} \psi^{\prime \prime} \delta v^{\prime \prime}+\omega_{v}^{2} \psi^{\prime \prime} \delta \psi^{\prime \prime}\right) d V \\
& +\int_{V} G\left(\dot{\omega}_{b}^{2} v^{\prime} \delta v^{\prime}+\dot{\omega}_{b} \dot{\omega}_{v} v^{\prime} \delta \psi^{\prime}+\dot{\omega}_{b} \dot{\omega}_{v} \psi^{\prime} \delta v^{\prime}+\dot{\omega}_{v}^{2} \psi^{\prime} \delta \psi^{\prime}\right) d V
\end{aligned}
$$

or

$$
\begin{aligned}
\delta W_{i}= & \int_{0}^{1} E\left(I_{x} v^{\prime \prime} \delta v^{\prime \prime}+I_{x v} v^{\prime \prime} \delta \psi^{\prime \prime}+I_{x v} \psi^{\prime \prime} \delta v^{\prime \prime}+I_{v} \psi^{\prime \prime} \delta \psi^{\prime \prime}\right) d z \\
& +\int_{0}^{l} G\left(J_{x} v^{\prime} \delta v^{\prime}+J_{x v} v^{\prime} \delta \psi^{\prime}+J_{x v} \psi^{\prime} \delta v^{\prime}+J_{v} \psi^{\prime} \delta \psi^{\prime}\right) d z
\end{aligned}
$$

where

$$
\begin{aligned}
& I_{x}=\int_{A} \omega_{b}^{2}(s) d A=\int_{A} y^{2} d A \\
& I_{x v}=\int_{A} \omega_{b}(s) \omega_{v}(s) d A \\
& I_{v}=\int_{A} \omega_{v}^{2}(s) d A \\
& J_{x}=\int_{A} \dot{\omega}_{b}^{2}(s) d A \\
& J_{x v}=\int_{A} \dot{\omega}_{b}(s) \dot{\omega}_{v}(s) d A \\
& J_{v}=\int_{A} \dot{\omega}_{v}^{2}(s) d A
\end{aligned}
$$

The external virtual work $\delta W_{e}$ can be written as

$$
\delta W_{e}=\int_{0}^{l} q_{y} \delta v(z) d z+\sum_{k} P_{y i} \delta v\left(z_{i}\right)
$$

where $q_{y}$ is the applied distributed load in the $y$ direction and $P_{y i}$ is the applied concentrated load acting at $z=z_{i}$ in the $y$ direction; $k$ is the number of concentrated loads acting on the box beam.

Equating $\delta W_{i}$ and $\delta W_{e}$ and integrating by parts, we obtain the governing differential equations as follows

$$
\begin{aligned}
& E I_{x} \frac{d^{4} v(z)}{d z^{4}}+E I_{x v} \frac{d^{4} \psi(z)}{d z^{4}}-G J_{z} \frac{d^{2} v(z)}{d z^{2}}-G J_{x v} \frac{d^{2} \psi(z)}{d z^{2}}=q_{y} \\
& E I_{x v} \frac{d^{4} v(z)}{d z^{4}}+E I_{v} \frac{d^{4} \psi(z)}{d z^{4}}-G J_{x v} \frac{d^{2} v(z)}{d z^{2}}-G J_{v} \frac{d^{2} \psi(z)}{d z^{2}}=0
\end{aligned}
$$

The boundary conditions for Eqs. (2.3.10) in the finite element context are:

For simply supported end:

$$
v=0
$$




$$
\psi=0
$$

For fixed end:

$$
\begin{aligned}
& v=0 \\
& v^{\prime}=0 \\
& \psi^{\prime}=0 \\
& \psi^{\prime}=0
\end{aligned}
$$

Based on those boundary conditions and the applied loading, the preceding differential equations can be solved, and the longitudinal normal and shear stresses due to shear lag can be found by Eq.(2.3.4).

For multicell beams, Roik and Sedlacek (1970) extended Reissner's hypothesis by introducing more than one shear lag warping function as shown in Fig. 2.7. These shear lag warping functions are linearly independent and the total shear lag effect is assumed to be due to their combined effect. The derivation of the relevant differential equations can be carried out similar to those for single cell box, but the mathematics of it would be quite tedious if written in expanded form. However, the equations may be written compactly using matrix notation. Let us define the displacement vector, $\left\{D_{s}\right\}$, and warping function vector, $\left\{\omega_{s}\right\}$, as

$$
\begin{aligned}
& \left\{D_{s}\right\}=\left[\begin{array}{llll}
u & \psi_{1} & \ldots & \psi_{m}
\end{array}\right]^{T}
\end{aligned}
$$

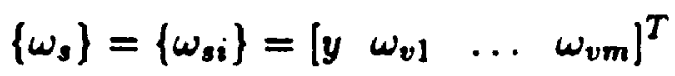

where $\omega_{v 1}$ to $\omega_{v m}$ are the shear lag warping functions as shown in Fig. 2.7, and $m$ is the total number of shear lag modes. The quantities $\psi_{1}$ to $\psi^{\prime} m$ are the $m$ components of displacement, each of which characterizes the mode of shear lag behaviour corresponding to the shear lag warping functions $\omega_{v 1}$ to $\omega_{v m}$. Following the sarne procedure as discussed above, the governing differential equations can be obtained as

$$
\left[C_{v}\right]\left\{D_{0}\right\}^{I V}-\left[J_{v}\right]\left\{D_{2}\right\}^{\prime \prime}=\{q\}
$$




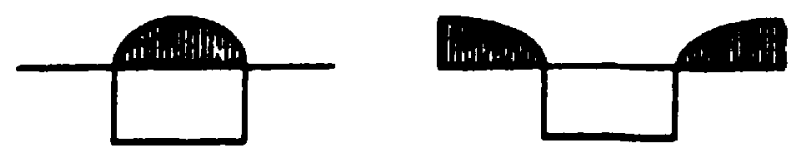

Single-cell section
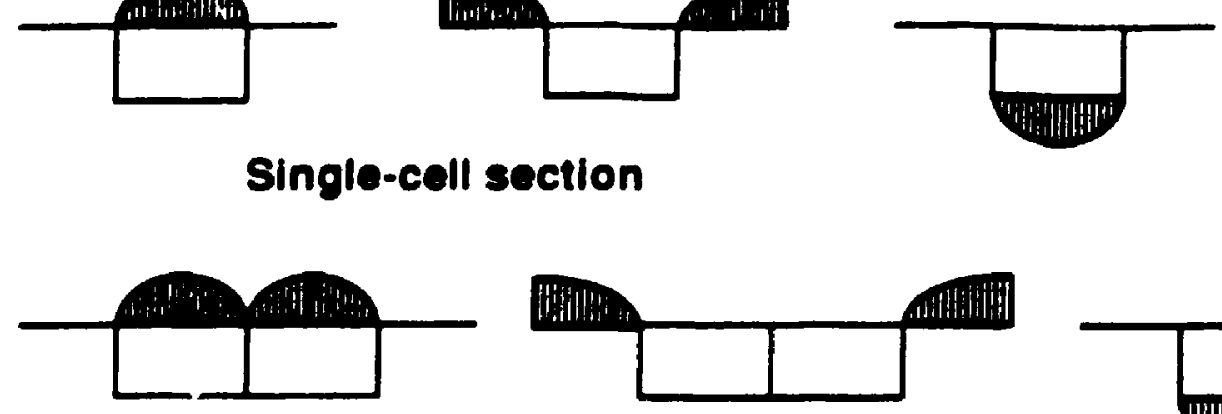

Two-call section

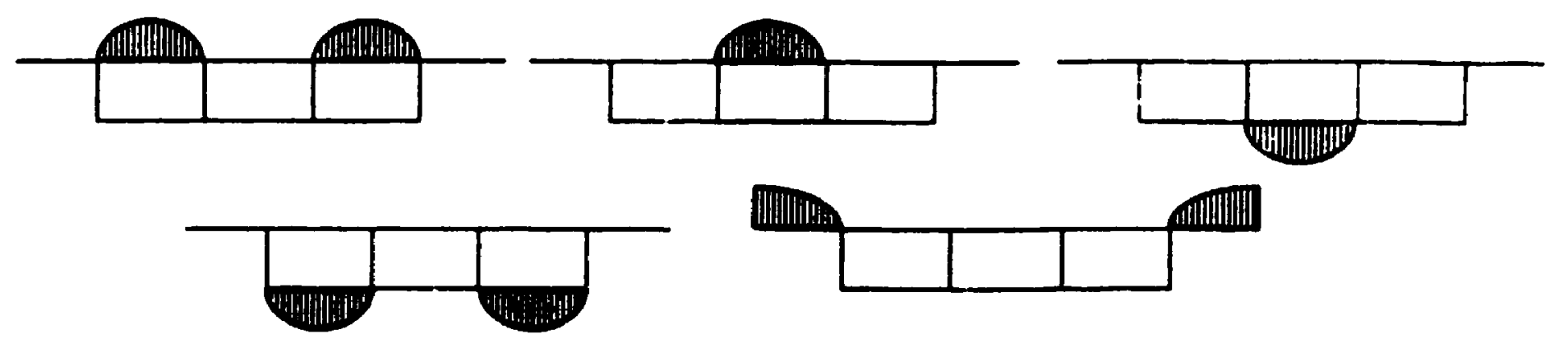

\section{Three-cell section}

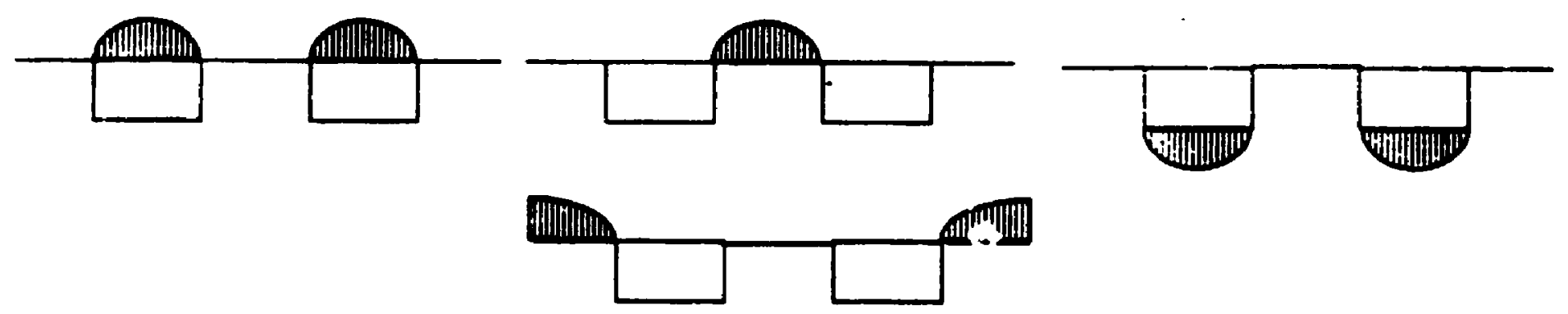

Two spine section

Fig. 2.7 Basic warping displacements for multicell box beam shear lag analysis.

$$
\begin{aligned}
& \text { where } \quad\left[C_{v}\right]=\int_{A} E\left\{\omega_{s}\right\}\left\{\omega_{s}\right\}^{T} d A \\
& {\left[J_{v}\right]=\int_{A} G\left\{\dot{\omega}_{s}\right\}\left\{\dot{\omega}_{0}\right\}^{T} d A} \\
& \{q\}=\left[\begin{array}{lllll}
q, & 0 & 0 & \ldots & 0
\end{array}\right]^{T}
\end{aligned}
$$

This system of simultaneous differential equations is coupled, as can be seen in Eqs. (2.3.10a) and (2.3.10b), but it can be uncoupled through an orthogonalization 
process which will be discussed in detail in the next chapter.

The longitudinal flexural normal stress and shear stress, including shear lag, can be calculated from the following

$$
\begin{aligned}
& \sigma_{2}=-E\left\{\omega_{1}\right\}^{T}\left\{D_{2}\right\}^{\prime \prime} \\
& \tau_{12}=-G\left\{\dot{\omega}_{2}\right\}^{T}\left\{D_{1}\right\}^{\prime}
\end{aligned}
$$

\subsection{Torsion}

\subsubsection{Pure Torsion}

Pure torsion, or St. Venant's torsion, occurs if a prismatic box beam is allowed to warp freely out of its cross sectional plane during twisting. In this case only shear stresses are induced in the member, and the relevant equations may be dervied as follows.

Consider a single-cell box beam subjected to a torsional moment $T$, in pure torsion as shown in Fig. 2.8. Assuming that shear stress, $\tau_{a}$, is uniformly distributed across the small wall thickness $t$, the shear flow $q_{s}=\tau_{,} t$ will be constant along the middle line of the cross section. Taking moment about the twist center, the equilibrium equation can be obtained as

$$
\oint \rho q s d s=T,
$$

where $\rho$ is the distance from the middle line of wall to the center of twist. Hence

$$
q_{s}=\tau_{s} t=\frac{T_{s}}{2 A_{c}}
$$

where $A_{c}$ is the area enclosed by the shear flow given by

$$
A_{c}=\frac{1}{2} \oint \rho d s
$$

Equating the strain energy of torsion to the work done by the torsional moment for a small element of length $d z$, we obtain

$$
\frac{1}{2} d z \oint_{t} \tau_{s} \gamma_{s} d s=\frac{1}{2} T_{s} \frac{d \theta_{z}}{d z} d z
$$


where $\gamma_{s}=\tau_{\imath} / G$.

Substituting Eq. (2.4.2) into Eq. (2.4.3), yields

$$
T_{0}=K_{0}, \frac{d \theta_{z}}{d z}=K_{0}^{\prime} \theta_{z}^{\prime}
$$

where $h$, is the torsional rigidity:

$$
K_{\bullet}=G J_{\text {. }}
$$

in which $J_{0}$ is torsional constant:

$$
J_{0}=\frac{4 A_{c}^{2}}{\oint \frac{d s}{t}}
$$

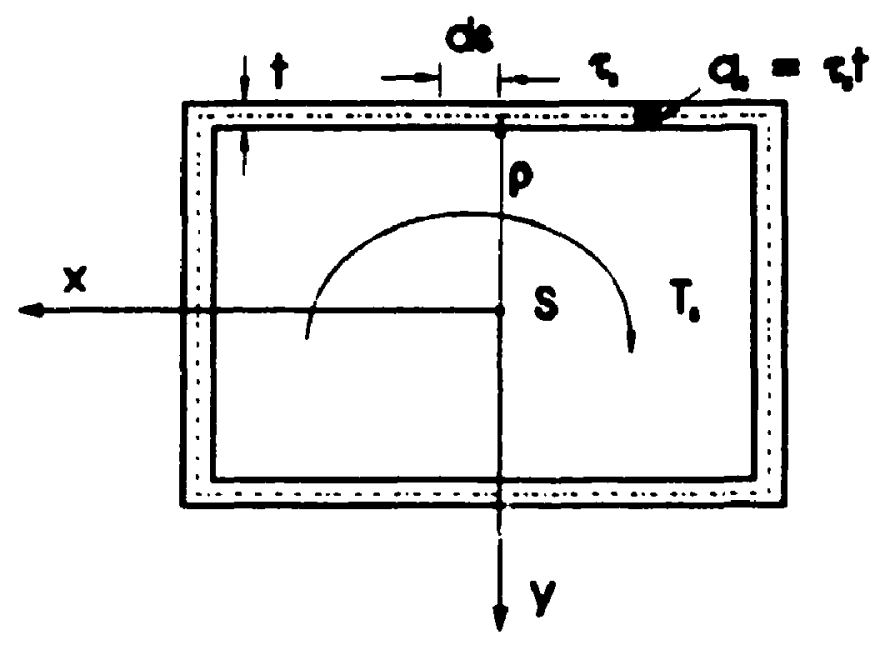

Fig. 2.8 Single-cell box beam subjected to pure torsion $T_{s}$.

For multicell box beams, we define $q_{s i}$ as the shear flow due to pure torsion in cell $i$. Based on the previous discussion for a single-cell box, for each cell, the following equation can be obtained:

$$
\frac{d \theta_{z}}{d z}=\frac{1}{2 A_{c 1} G} \oint_{2} \frac{q_{s i}}{t} d s
$$

where $A_{c t}$ is the area enclosed by the middle line of cell $i$. However, for each cell we must account for the shear flow in the adjacent cells. This can be achieved by subtracting the shear flow in the adjacent cells from that of the cell under consideration along their common wall. For example, for a three cell section as shown in 
Fig. 2.9, the set of simultaneous equations corresponding to $\mathrm{Eq} .(2.4 .7)$ will have the following form:

$$
\begin{gathered}
q_{s 1} \oint_{1} \frac{d s}{t}-q_{s 2} \int_{12} \frac{d s}{t}=2 . A_{c 1} G \frac{d \theta_{z}}{d z} \\
-q_{s 1} \int_{12} \frac{d s}{t}+q_{s 2} \oint_{2} \frac{d s}{t}-q_{s 3} \int_{23} \frac{d s}{t}=2 A_{c 2} G \frac{d \theta_{z}}{d z} \\
-q_{s 2} \int_{23} \frac{d s}{t}+q_{s 3} \oint_{3} \frac{d s}{t}=2 A_{c 3} G \frac{d \theta_{z}}{d z}
\end{gathered}
$$

where the subscripts 1,2 and 3 refer to the three cells and the subscripts 12 and 23 denote the common wall between cells $1-2$ and $2-3$, respectively.

In addition, from the equilibrium condition

$$
T_{s}=\sum_{1} 2 A_{c 1} g_{s 1}
$$

where $T$, is the total applied torque. By solving Eqs. (2.4.8) to (2.4.9) for $q_{01}, q_{12}$, $q_{s 3}$ and $\theta_{2}^{\prime}$, the shear stress due to pure torsion can be found from $r_{s}=q_{s} / t$. The torsional constant for multicell box beam can be found by

$$
J_{s}=\frac{2}{G \theta_{z}^{\prime}} \sum q_{91} A_{c 1}
$$

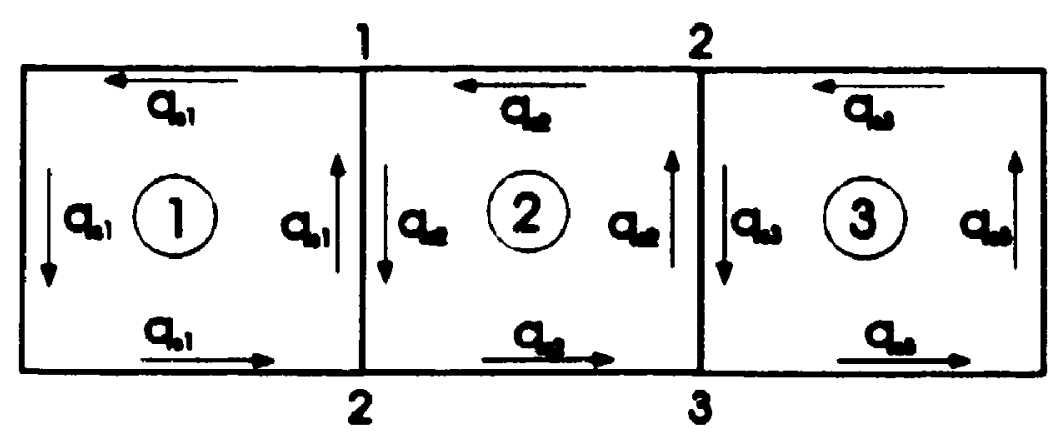

Fig. 2.0 Shear flows due to pure torsion in multicell box beam. 


\subsubsection{Torsional Warping}

For a thin-walled beam subjected to twisting, if the axial displacement $w$ is restrained, i.e. if warping is prevented, normal stress $\sigma_{8}=\sigma_{\omega}$ will occur in the longitudinal direction. This longitudinal displacement due to restrained twisting is called torsional warping. In torsional warping analysis, it is assumed that there is no distortion of the cross section. Based on Vlasov's theory, the torsional warping displacement can be written as

$$
w(s, z)=-\omega(s) \theta^{\prime}
$$

where $\omega(s)$ is the warping fuction and was originally referred to by Vlasov as a sectorial coordinate. This function represents the warping displacements due to unit relative twisting $\theta_{z}^{\prime}$ of adjacent cross sections.

Mathematically $\omega$ may be written as

$$
\omega=\omega_{0}+\int_{0}^{s}\left(\rho-\frac{\bar{q} s}{t}\right) t d s
$$

where $\omega_{0}$ is an unknown torsional warping function at the origin $s=0, \bar{q}_{0}$ is the shear flow due to unit $\theta_{z}^{\prime}$ in pure torsion and $\rho$ is defined in Fig. 2.10.

The value of $\omega$ depends upon the location of pole $P\left(x_{p}, y_{p}\right)$ and upon the value of $\omega_{0}$, which in turn depends upon the position of the origin of the $s$ coordinate, $O_{1}$. The theory is simplified by a suitable choice of points $P$ and $O_{1}$, for example, with $P$ at the shear center $S$ and $O_{1}$ in a position which yields

$$
\int_{A} \omega d A=\int_{g} \omega t d s=0
$$

Notice that Eq. $(2,4.13)$ is akin to the first moment of area.

The shear center has the property that, when the resultant transverse shear forces on the section act through it, there is no torsion on the section. This results in the uncoupling of the equations of flexure and torsional warping. The above sectorial coordinate is called the normalized sectorial coordinate $\omega$. The procedure for determining the position of the shear center and the normalized sectorial coordinate $\omega$ is as follows. 
Consider Fig. 2.10 and choose an arbitrary pole $P\left(x_{p}, y_{p}\right)$ and an arbitrary origin $O_{1}$ for the curvilinear coordinate $s$ and calculate the sectorial coordinate wp as defined in Eq. (2.4.12), then the coordinates of the shear center $S$, referred to the principal axes of the cross section, are given by

$$
\begin{gathered}
x_{0}=\frac{I_{y w p}}{I_{x}}+x_{p} \\
y_{0}=-\frac{I_{x-p}}{I_{y}}+y_{p}
\end{gathered}
$$

where

$$
\begin{aligned}
& I_{x \omega p}=\int_{A} \omega_{p} x d A \\
& I_{y \omega p}=\int_{A} \omega_{p} y d A
\end{aligned}
$$

Once the position of the shear center $S$ is known, the diagram of $\omega_{p}$ may be redrawn, using $S$, and not $P$, as the pole. Accordingly, the sectorial coordinate with respect to the shear center as the pole is given by

$$
\omega_{s}=\int_{0}^{*}\left(\rho_{s}-\frac{\bar{q}_{s}}{t}\right) t d s
$$

To eliminate $\omega_{0}$ from Eq. (2.4.12), we shift the origin $O_{1}$ to point $O_{2}$ and determine the location of $\mathrm{O}_{2}$ from the relationship

$$
\int_{A} \omega d A=0
$$

The latter is satisfied if

$$
\omega=\omega_{\mathrm{s}}+\omega_{0}
$$

where $\quad \omega_{0}=-\frac{1}{A} \int_{A} \omega_{s} d A$

This defines the distance between points $O_{1}$ and $O_{2}$ as follows

$$
O_{1} O_{2}=\frac{\omega_{0}}{\rho_{o s}}
$$

where $\rho_{0}$ is the perpendicular distance from the shear center $S$ to line $O_{1} O_{2}$. Note that in general, $\omega_{0}=0$ if the origin $s$ is located on an axis of symmetry in the cross section. 


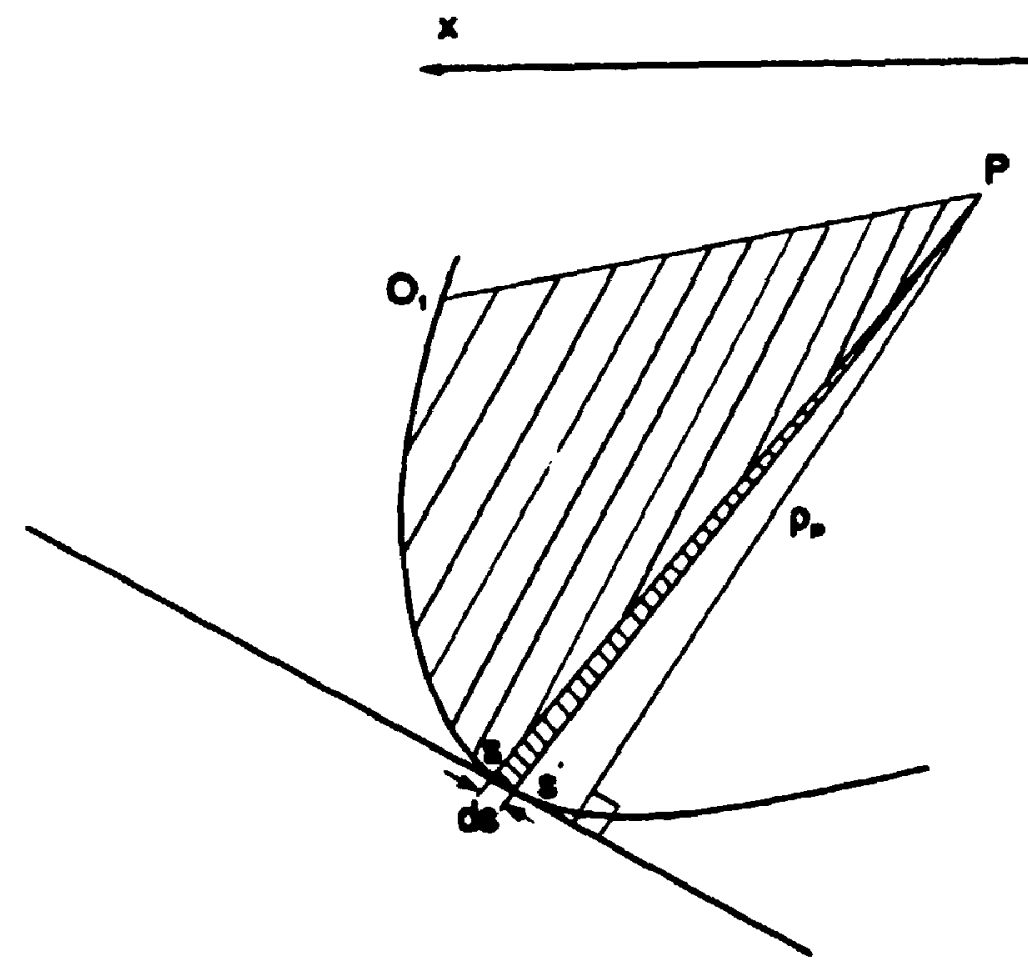

Fig. 3.10 Definition of sectorial coordinate $w(s)$.

The calculation of normal stresses due to restrained warping requires that the so-called torsional warping constant, $I_{w}$, be known. This constant can be determined using

$$
I_{\omega}=\int_{A} \omega^{2} d A
$$

From Eq. (2.4.11), the longitudinal normal strain $\epsilon_{z}$ can be obtained as

$$
\epsilon_{z}=\frac{\partial w(s, z)}{\partial z}=-w(s) \theta_{z}^{\prime \prime}
$$

The longitudinal normal stress due to torsional warping, $\sigma_{\omega}$, which is similar to the flexural normal stress $\sigma_{b}$ is given by the following equation:

$$
\sigma_{\omega}=-E \omega \frac{d^{2} \theta_{z}}{d z^{2}}=\frac{M_{\omega}}{I_{\omega}} \omega
$$

where $M_{\omega}$ is called the torsional warping moment or bimoment:

$$
M_{\omega}=-E I_{\omega} \frac{d^{2} \theta_{z}}{d z^{2}}
$$

The torsional warping shear flow $q_{\omega}$ can be defined similarly to the shear flow $q_{b}$ due to bending, i.e.

$$
q_{\omega}=\tau_{\omega} t
$$


where $\tau_{\omega}$ is the torsional warping shear stress and can be determined as follows.

Consider the infinitesimal element in Fig. 2.11, the warping normal stress $\sigma_{\omega}$ and shear stress $\tau_{w}$ acting on this element satisfy the eqilibrium condition

$$
\frac{\partial \sigma_{\omega} t}{\partial z}+\frac{\partial \tau_{\omega} t}{\partial s}=0
$$

Substituting Eqs. (2.4.23) and (2.4.25) into Eq. (2.4.26) and integrating with respect to $s$ gives

$$
q_{\omega}=-\int_{0}^{0} \frac{\partial \sigma_{\omega} t}{\partial z} d s=-E \frac{d^{3} \theta_{z}}{d z^{3}}\left(\int_{0}^{e} \omega t d s+C\right)
$$

where $C$ is an integration constant and can be obtained from the condition

$$
\oint \frac{q_{\omega}}{G t} d s=0
$$

For single-cell box beam, $C$ is given by

$$
C=-\frac{\oint[1 /(G t)]\left(\int_{0}^{s} \omega t d s\right) d s}{\oint \frac{d s}{G t}}
$$

The same procedure can be applied to a multicell box beam by considering

$$
q_{\omega 0}=-E\left(\frac{d^{3} \theta_{z}}{d z^{3}}\right) \int_{0}^{s} \omega t d s
$$

for an imaginary open section and then using Eqs. (2.2.15) and (2.2.16) to find the corresponding statically indeterminate shear flows $q_{w 1}$.

The Wagner's torsional moment $T_{\omega}$, also called the secondary torsion, is defined as

$$
T_{\omega}=\int_{A} q_{\omega} \rho d s=-E \frac{d^{3} \theta_{z}}{d z^{3}} \int_{A} \omega^{2} t d s
$$

or

$$
T_{\omega}=-E I_{\omega} \frac{d^{3} \theta_{z}}{d z^{3}}
$$

Let us define the first moment of warping function as

$$
S_{\omega}=\int_{0}^{s} \omega t d s
$$

then the shear stress $\tau_{\omega}$ due to Wagner's torsion can be written as

$$
\tau_{\omega}=\frac{q_{\omega}}{t}=\frac{T_{\omega}}{I_{\omega} t} S_{\omega}
$$



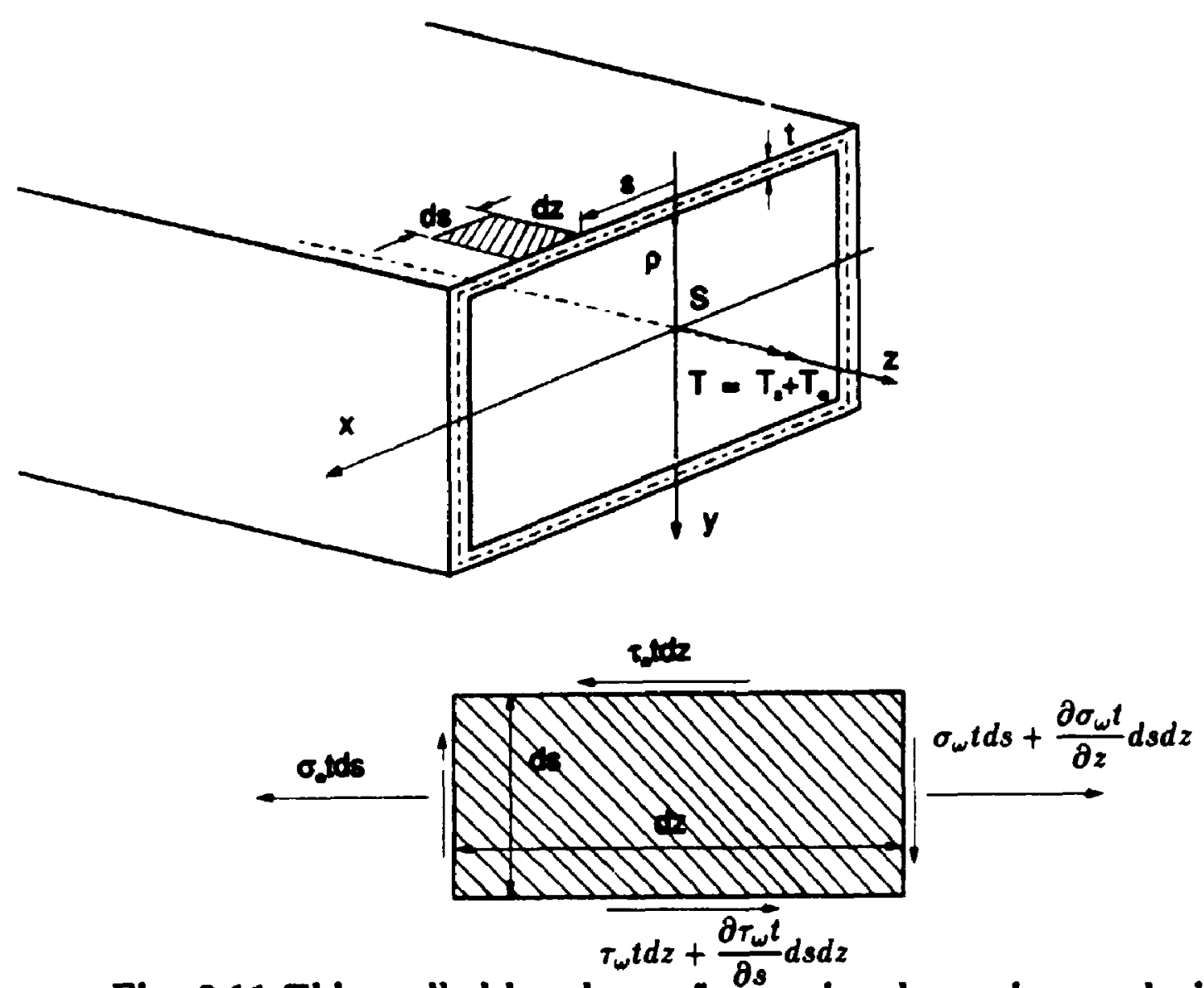

Fig. 2.11 Thin-walled box beam for torsional warping analysis.

Finally, it should be noted that the total torsional moment, $T$, is

$$
T=T_{s}+T_{\omega}
$$

Thus by substituting for $T_{3}$ from Eq. (2.4.4) and for $T_{\omega}$ for Eq. (2.4.32) into Eq. (2.4.35), the governing differential equation of torsional warping may be written as

$$
E I_{\omega} \frac{d^{4} \theta_{z}}{d z^{4}}-G J_{2} \frac{d^{2} \theta_{z}}{d z^{2}}=-\frac{d T}{d z}
$$

The solution of this equation will depend on the loading and boundary conditions and will be discussed in detail in the next chapter.

\subsection{Distortion}

\subsubsection{Distortion of a Single-Cell Beam}

Box beams without diaphragms or with insufficient intermediate diaphragms are susceptible to cross sectional distortion under torsional loading conditions. Distor- 
tional effects comprise distortional warping and transverse bending, which have to be superimposed upon the effects of longitudinal bending and torsional warping in order to completely assess the state of stresses in a box. Distortion can be regarded as differential torsional deformation of the individual parts of the cross section. The elastic resistance with which box beams oppose distortion can be divided into warping resistance, torsional resistance (as in torsional warping), and transverse bending resistance of the cruss section. This transverse bending resistance is porportional to the amount of distortion occuring. If elastic diaphragms are present, they can be included in the analysis as will be shown later.

In order to have a physical understanding of the distortional behaviour of box beams, a single-cell monosymmetric box beam in Fig. 2.12 is subjected to a vertically distributed load $p$ with an eccentricity $e$. This applied force may be resolved into a set of loads $p / 2$ causing only flexure and a torsional moment $m_{t}=p e$, with the latter being further divided into loads causing torsion and distortion, as shown in Fig. 2.12 (Nakai and Yoo 1988). The total response of this structure may be obtained by analyzing for flexure, torsion and distortion separately and then superimposing them.

The coordinate axes $(x, y, z)$ are taken at the distortional center $D$, while the curvilinear coordinate $s$ once again runs along the perimeter of the cross section. When the cross section of the box beam distorts, the distortion can be represented by the angular change $\beta$ at each corner of the box, as shown in Fig. 2.13. Sirnilar to the torsional warping displacement, the longitudinal distortional warping displacement may be defined as

$$
w(s, z)=-\omega_{d}(s) \beta^{\prime}(z)
$$

where $\omega_{d}(s)$ is the distortional warping function, which is given by

$$
\omega_{d}=\int_{0}^{s}\left[\rho_{d}(s)-\frac{\bar{q}_{d}}{t}\right] t d s+\omega_{d 0}
$$

in which $\rho_{d}(s)$ is the perpendicular distance from the distortional center $D$ to the tangent at point $(x, y) ; \bar{q}_{d}$ is the shear flow due to unit $\beta^{\prime}(z)$, similar to $\bar{q}_{s}$ in St. 

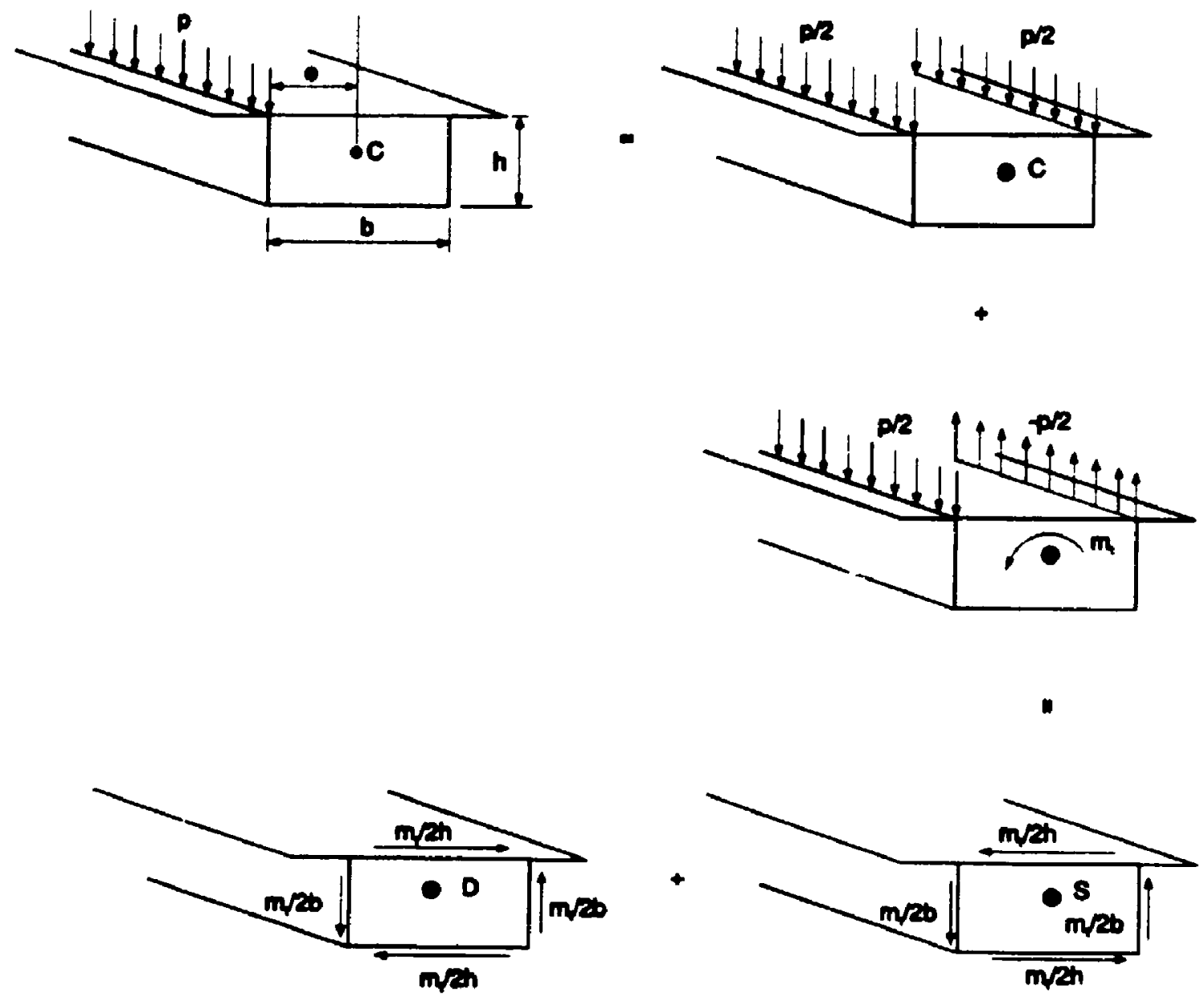

Fig. 2.12 Single-cell box girder subjected to eccentric loading.

Venant's torsion, and is given by

$$
\bar{q}_{d}=\frac{\int_{0}^{s} \rho_{d}(s) d s}{\oint \frac{d s}{t}}
$$

where $\omega_{d 0}$ is the integration constant which can be found in the same way as for torsional warping.

For the case where the displacement $w(s, z)$ is restrained, longitudinal normal and shear stress will be produced in the box section. The corresponding stress resultants can be defined as shown in Fig. 2.14, where $M_{d \omega}$ and $T_{d \omega}$ are the bimoment and torsional moment due to distortional warping, respectively. Note that these are similar in definition to those for torsional warping theory. Let $v_{s}(s, z)$ denote the displacement along $s$ in the walls of the cross section. According to the theory of 


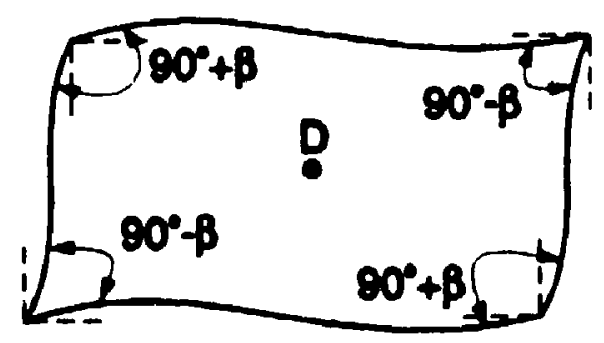

Fig. 2.13 Definition of diatortional angle $\beta$.

elasticity, the normal strain $\epsilon_{z}$ and shear strain $\gamma_{s z}$ may be obtained from

$$
\begin{gathered}
\epsilon_{z}=\frac{\partial w(s, z)}{\partial z} \\
\gamma_{s z}=\frac{\partial w(s, z)}{\partial s}+\frac{\partial v_{s}(s, z)}{\partial z}
\end{gathered}
$$

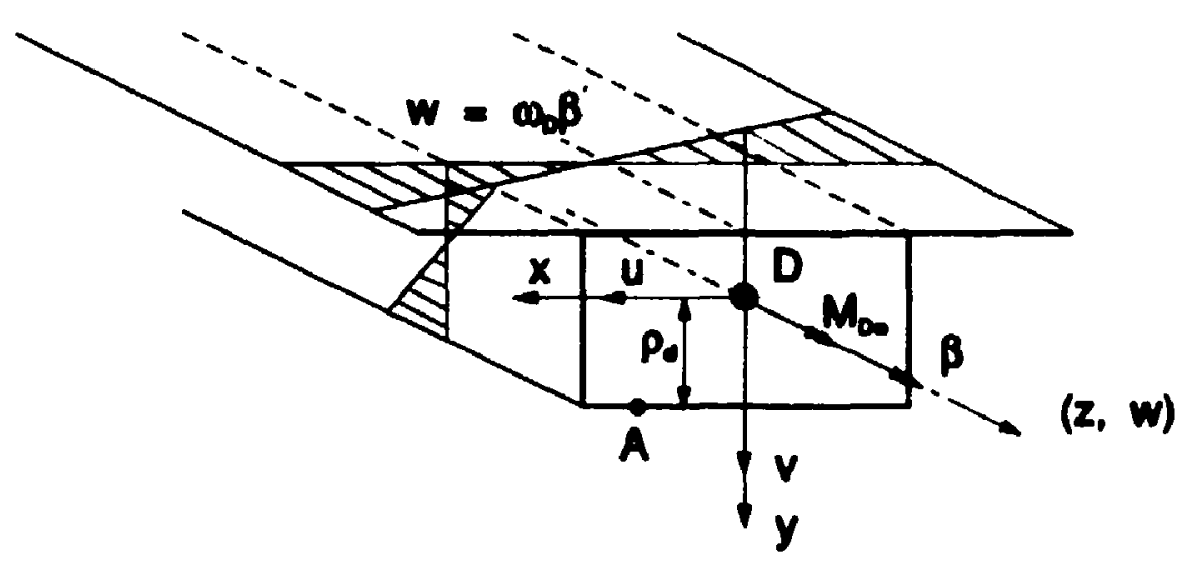

Fig. 2.14 Definition of stress resultants and displacements.

The displacement component $v_{s}(s, z)$ can be written by separation of variables as the product of the angular distortion $\beta(z)$ and the perpendicular distance $\rho_{d}(s)$ from the distortional center $D$ of the cross section to a point $A$ as shown in Fig. 2.14 , i.e.

$$
v_{s}(s, z)=\beta(z) \rho_{d}(s)
$$

Substituting Eq. (2.5.1) into Eq. (2.5.4), the longitudinal normal strain $\epsilon_{z}$ becomes

$$
\epsilon_{z}=\frac{\partial w(s, z)}{\partial z}=-\omega_{d}(s) \beta^{\prime \prime}(z)
$$


and the corresponding longitudinal normal stress is

$$
\sigma_{z}=\sigma_{d}=E \epsilon_{z}=-E \omega_{d}(s) \beta^{\prime \prime}(z)
$$

Since distortional stresses are self-equilibrating, the following equilibrium equations may be written:

$$
\begin{aligned}
N_{z} & =\int_{A} \sigma_{d} d A=0 \\
M_{x} & =\int_{A} \sigma_{d} y d A=0 \\
M_{y} & =\int_{A} \sigma_{d} x d A=0
\end{aligned}
$$

These three equations can be used to determine the coordinates $x_{d}$ and $y_{d}$ of the distortional center and the origin of coordinate $s$ for distortion.

The distortional bimoment can be calculated by the following equation, similar to that for torsional warping:

$$
M_{d \omega}=-E I_{d \omega} \frac{d^{2} \beta}{d z^{2}}
$$

where $I_{d w}$ is the distortional warping constant which is given by

$$
I_{d \omega}=\int_{A} \omega_{d}^{2} d A
$$

From Eq. (2.5.10), Eq. (2.5.8) can be written in the same form as the torsional warping equation. Thus

$$
\sigma_{d}=\frac{M_{d \omega}}{I_{d \omega}} \omega_{d}
$$

The shear flow $q_{d w}$ due to distortion is given by

$$
q_{d \omega}=\tau_{d \omega} t=\frac{S_{d \omega}}{I_{d \omega}} T_{d \omega}
$$

in which $T_{d w}$ is the torsional moment due to distortional warping and is defined by

$$
T_{d \omega}=\frac{\partial M_{d \omega}}{\partial z}=-E I_{d \omega} \frac{d^{3} \beta}{d z^{3}}
$$

and $S_{d \omega}$ is the static moment of the distortional function $\omega_{d}$, given by

$$
S_{d \omega}=\int_{0}^{s} \omega_{d} t d s+C_{2}
$$


The integration constant $C_{2}$ can be directly determined from the condition

$$
\oint \frac{q d \omega}{G t} d s=0
$$

The transverse bending due to distortion of the cross section will produce the normal strain $\epsilon_{\text {a }}$ in transverse direction which is given by

$$
\epsilon_{s}=n \frac{d^{2} v_{n}(s)}{d s^{2}}=n \frac{d^{2} \bar{v}_{n}(s)}{d s^{2}} \beta
$$

where $n$ is the normal coordinate in the direction of the thickness of each wall, $v_{n}(s)$ is the displacement of the walls in direction $n$ due to distortion, and $\bar{v}_{n}(s)$ is the displacement of the walls due to unit $\beta$. The corresponding normal stress $\sigma_{\theta}$ in the transverse direction may be calculated as

$$
\sigma_{s}=E_{s} \epsilon_{s}=E_{s} n \frac{d^{2} \bar{v}_{n}(s)}{d s^{2}} \beta
$$

The governing differential equations of distortion can be developed by the principle of virtual work. The internal virtual work $\delta W_{i}$ is given by

$$
\delta W_{1}=\int_{V}\left(\sigma_{d} \delta \epsilon_{d}+\tau_{d} \delta \gamma_{d}+\sigma_{s} \delta \epsilon_{s}\right) d V
$$

Substituting Eqs. (2.5.8) and (2.5.18) into the above equation, we have

$$
\delta W_{i}=\int_{l}\left(\beta^{\prime \prime} E I_{d \omega} \delta \beta^{\prime \prime}+\beta^{\prime} G J_{d} \delta \beta^{\prime}+\beta E_{s} K_{d} \delta \beta\right) d z
$$

where $J_{d}$ is the distortional constant which is similar to the torsional constant given in Eq. (2.4.6), and $K_{d}$ is the distortional stiffness of the cross section. The last two quantities are defined by

$$
\begin{aligned}
J_{d} & =\int_{A}\left(\frac{\bar{q}_{d}}{t}\right)^{2} d A \\
K_{d} & =\oint_{s} \frac{\bar{M}_{g}^{2}(s)}{E_{g} I_{g}(s)} d s
\end{aligned}
$$

in which $\bar{M}_{2}(s)$ is the bending moment distribution in the walls due to unit deformation $\beta$, and $I_{s}(s)$ and $E$, are the moment of inertia and elastic modulus of the transverse frame members, respectively. 


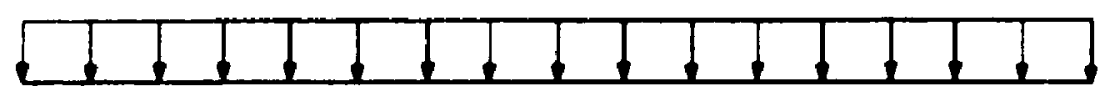

(a) Distortional loed $(q=m / 2)$

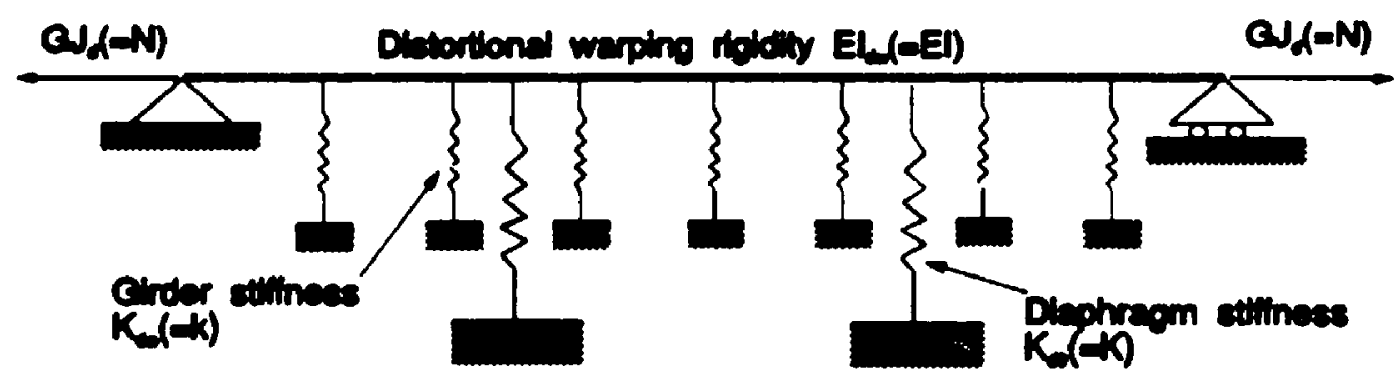

(b) Equivalem baam

Fig. 2.15 Beam-column on elastic foundation analogy for distortional analysis.

The external virtual work is given by

$$
\delta W_{e}=\int_{l} \frac{m_{t}}{2} \beta d z
$$

Equating $\delta W_{i}$ and $\delta W_{e}$, and integrating by parts, we have

$$
E I_{d \omega} \frac{d^{4} \beta}{d z^{4}}-G J_{d} \frac{d^{2} \beta}{d z^{2}}+K_{d} \beta=\frac{m_{t}}{2}
$$

The above equation is the governing differential equation for distortional warping which is similar to the equation describing the flexural deflection $w$ of a beam-column on an elastic foundation with flexural rigidity $E I$, axial tensile force $N$, foundation modulus $k$, and uniformly distributed load $q$. The equation of such a beam-column has the form

$$
E I \frac{d^{4} w}{d z^{4}}-N \frac{d^{2} w}{d z^{2}}+k w=q
$$

Note that $a$ box beam subjected to the distortional moment $m_{t} / 2$ as in Fig. 2.15 is generally supported not only by the imaginary elastic foundation with stiffness $K_{d}$ but also by the diaphragms which are akin to concentrated spring supports of stiffness $K_{d 0}$. The detailed solution of the above equation will be discussed in the next chapter. 


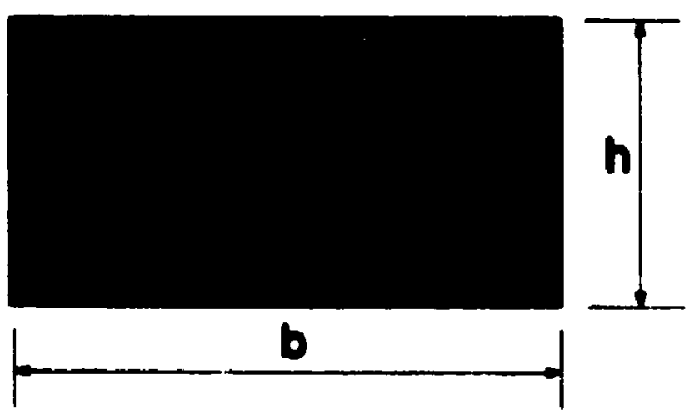

(a) Eotid - plan mpe
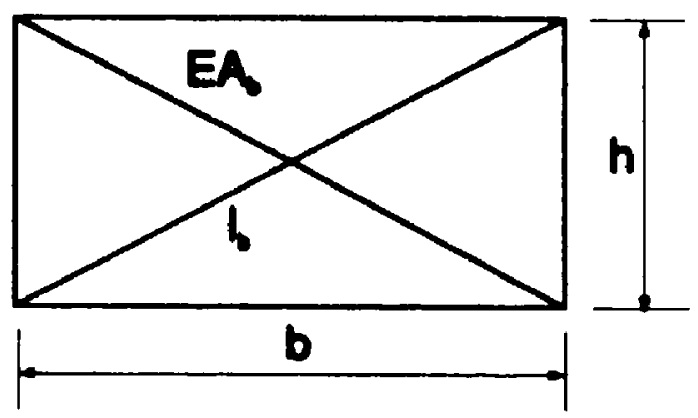

(b) Truas $\times$ spe
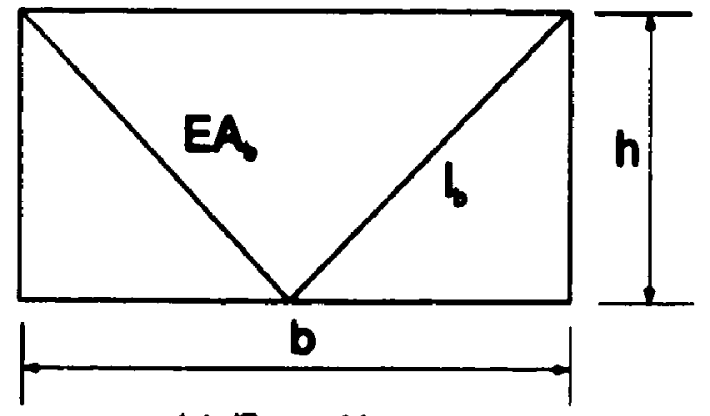

(c) Trues V typo

\section{Fig. 2.16 Types of intermediate diaphragms.}

The diaphragm stiffness $K_{d 0}$ can be estimated according to the type of diaphragm (Nakai and Yoo 1988). Fig. 2.16 shows the various types of diaphragms, and their related stiffnesses are as follows

For plate-type diaphragms,

$$
K_{d 0}=G t_{d} b h
$$

where $G, t_{d}, b$ and $h$ are the shear modulus, thickness, breadth and length of the diaphragm, respectively. For truss-type diaphragms,

$$
K_{d 0}=\left\{\begin{array}{l}
\frac{2 E A_{b} b^{2} h^{2}}{l_{b}^{3}} \\
\frac{E A_{b} b^{2} h^{2}}{2 l_{b}^{3}}
\end{array}\right.
$$


where $E, A_{b}$ and $l_{b}$ are the Young's modulus, cross-sectional area and length of the truss member, respectively; and $b$ and $h$ are the width and the depth of the box, respectively.

\subsubsection{Distortion of Multicell Box Beams}

Distortion of multicell beams can be analyzed similarly to that of single-cell beams but more than one distortional mode should be considered. For single spine beams, the number of distortional modes will depend on the number of cells in the cross section, as illustrated in Fig. 2.17. These basic distortional modes are linearly independent, therefore, the total distortional effect for multicell beams can be computed by combining the basic modes. Due to the presence of several distortional modes in multicell beams, it is not practical to find a single distortional center for the entire section. As indicated in Fig. 2.17, the basic distortional modes are relative joint displacements and are defined with respect to a reference wall in any cell. Based on the coordinate system shown, the governing differential equations for the various distortional modes will be coupled. In the next chapter, a two step orthogonalization process will be presented to uncouple these differential equations.

The generalized displacements that describe the total deformations of the box are given by the vector

$$
\left\{D_{d}\right\}=\left[\begin{array}{lllllll}
\hat{w} & u & v & \theta_{z} & \beta_{1} & \ldots & \beta_{n}
\end{array}\right]^{T}
$$

where $\hat{u}=\int w d x$; the notation $\hat{w}$ is used instead of $w$ merely for the sake of writing the governing equations in a unified compact form. the vector $\left[\begin{array}{lllll}\hat{w} & u & v & \theta_{z}\end{array}\right]^{T}$ is the four orthogonalized non-distortional displacement components, i.e. $w$ is the axial displacement, $u$ and $v$ are the lateral displacements in the $x$ and $y$ directions, respectively, and $\beta_{1}$ to $\beta_{n}$ are relative rotations in the plane of the cross-section which define the basic distortional modes as in Fig. 2.17, $n$ is the total number of linearly independent distortional modes.

For a point $(x, y)$ on a wall of the box, the generalized warping vector is given 

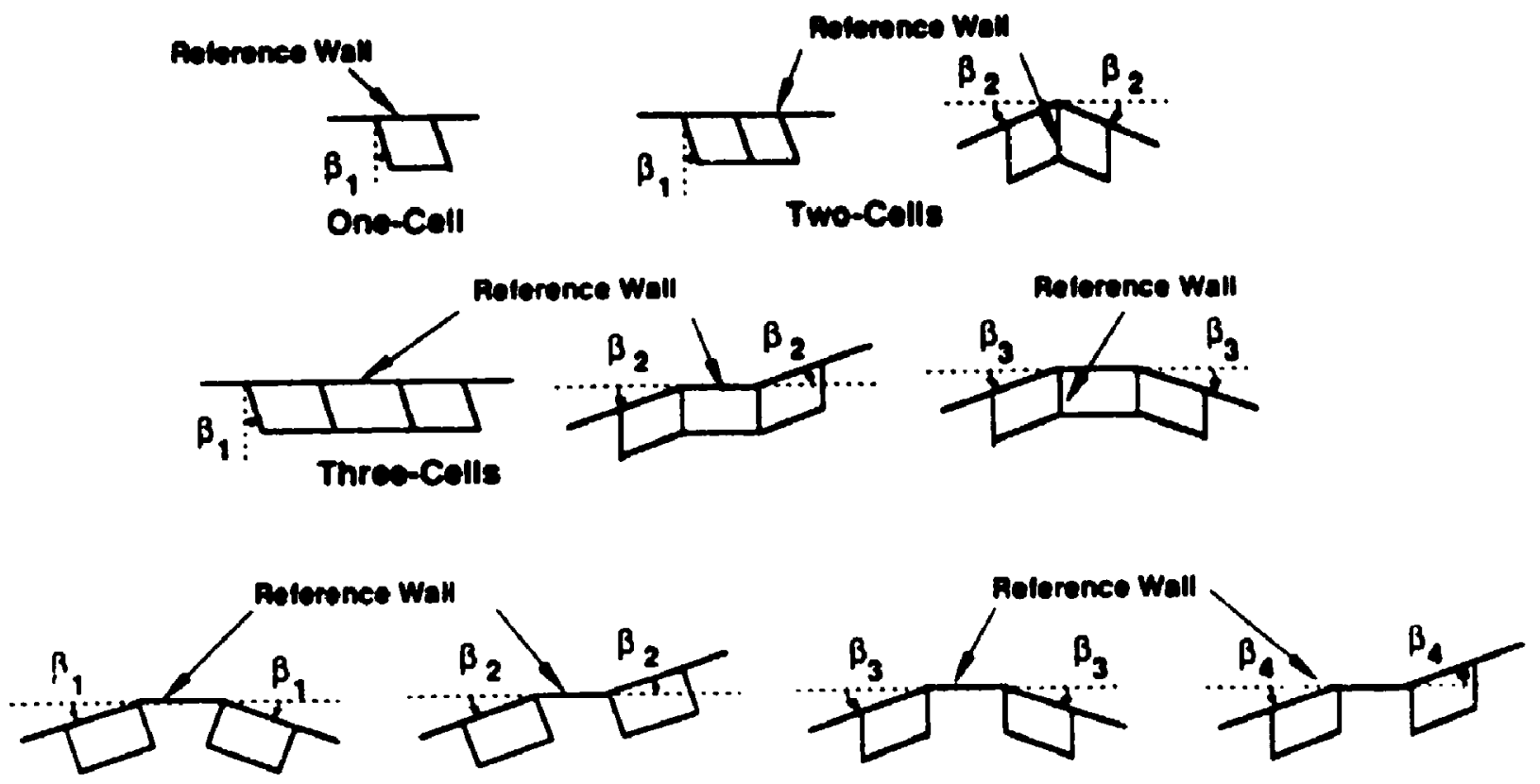

Two Spinos

Fig. 2.17 Basic distortional modes for various cross sections.

by

$$
\left\{\omega_{d}\right\}=\left\{\omega_{d i}\right\}=\int_{0}^{s}\left(\left\{r_{d}\right\}-\left\{\bar{r}_{d}\right\}\right) d s=\left\{\begin{array}{c}
1 \\
x \\
y \\
\omega \\
\omega_{d 1} \\
\cdot \\
\cdot \\
\omega_{d n}
\end{array}\right\}
$$

where $\omega$ is the torsional warping function and $\omega_{d 1}$ to $\omega_{d n}$ are the distortional warping functions. The vectors $\left\{r_{d}\right\}$ and $\left\{\bar{r}_{d}\right\}$ are given by

$$
\begin{gathered}
\left\{r_{d}\right\}=\left[\begin{array}{llllllll}
0 & \cos \alpha & \sin \alpha & \rho & r_{1}, & \ldots & , & r_{n}
\end{array}\right]^{T} \\
\left\{\bar{\tau}_{d}\right\}=\left[\begin{array}{llllllllll}
0 & 0 & 0 & \bar{\tau}_{s v} & \bar{\tau}_{1}, & \ldots & \bar{\tau}_{n}
\end{array}\right]^{T}
\end{gathered}
$$


in which $\alpha$ is the angle between the $x$ axis and the tangent at point $(x, y) ; \rho$ is the perpendicular distance from the shear center to the same tangent; $r_{i}$ is the distance from the reference wall defining rotation $\beta_{i}$, as shown in Fig. 2.17 , to the tangent to the wall element experiencing tangential movement in distortion, and $F$ is a shear stress distribution function obtained by solving the following simultaneous equations

$$
-\left(\bar{\tau}_{i} t\right)_{i-1} \int_{i-1, i} \frac{d s}{t}+\left(\bar{\tau}_{i} t\right)_{i} \oint_{i} \frac{d s}{t}-\left(\bar{\tau}_{i} t\right)_{i+1} \int_{i, i+1} \frac{d s}{t}=\int_{i} r_{i} d s
$$

where $t$ is the wall thickness and the index $i$ varies from one to $n$. Eq. (2.5.31) is a compatibility equation derived from the consideration that no slip or relative longitudinal displacement may occur in the cross section of a closed section. Transverse bending moments are related to rotation $\beta_{i}$ by treating the box cross-section as a frame of unit length along the span, and analyzing it by the standard stiffness method. The governing differential equations of a thin-walled box beam, including extension, biaxial bending, torsion, torsional warping, distortion and distortional warping can be derived by the principle of virtual work, and are of the form

$$
\left[C_{d}\right]\left\{D_{d}^{I V}\right\}-\left[J_{d}\right]\left\{D_{d}^{\prime \prime}\right\}+\left[H_{d}\right]\left\{D_{d}\right\}=q_{x}\left\{r_{x}^{*}\right\}+q_{y}\left\{r_{y}^{*}\right\}+\int_{s} q_{z}^{\prime}\{\omega\} d s
$$

where

$$
\begin{gathered}
{\left[C_{d}\right]=\int_{A} E\left\{\omega_{d}\right\}\left\{\omega_{d}\right\}^{T} d A} \\
{\left[J_{d}\right]=\int_{A} G\left\{\bar{\tau}_{d}\right\}\left\{\bar{\tau}_{d}\right\}^{T} d A} \\
{\left[H_{d}\right]=\oint_{s} \frac{\left\{M_{d s}(s)\right\}\left\{M_{d s}(s)\right\}^{T}}{E_{s} I_{s}(s)} d s}
\end{gathered}
$$

in which $\left\{M_{d s}(s)\right\}$ is the vector of transverse bending moments corresponding to unit values of the components of vector $\left\{D_{d}\right\} ; q_{x}, q_{y}$ and $q_{x}$ are the distributed loads acting on the box in the $x, y$ and $z$ directions, respectively; $\left\{r_{z}^{*}\right\}$ and $\left\{r_{y}^{*}\right\}$ are vectors describing the position of the loads $q_{x}$ and $q_{y}$ on the cross section and are measured from the shear center. Distortional stiffnesses due to intermediate elastic diaphragms for different distortional modes can also be included as it will be discussed in next section. 


\subsubsection{Transverse Bending}

Transverse bending is caused by the distortion of the cross section and by loads applied between the webs. Figure 2.18 shows the transverse bending of a multicell cross section, including the elastic diaphragms, due to different distortional modes. Transverse bending due to loads applied between the webs is shown in Fig. 2.19. The latter effect may be treated by considering a unit lenght of the box as a Vierendeel frame. The total transverse bending can then be found by superposing of the two effects.

\subsection{Summary of Governing Equations of Multicell Box Beams}

In the previous sections each structural action in a thin-walled beam was discussed separately. Similar procedures can be used to develop the general governing equations which account for their combined effects. Following Maisel (1982), in the present treatment, the interaction between distortional and shear lag effects will be neglected. The generalized displacement fields describing the beam deformations corresponding to all the above actions may be defined by

$$
\{D\}=\left[\begin{array}{lllllllllllll}
\hat{w} & u & v & \theta_{z} & \beta_{1} & \beta_{2}, & \ldots & \beta_{n} & \psi_{1} & \psi_{2}, & \ldots & , \psi_{m}
\end{array}\right]^{T}
$$

where $\left[\begin{array}{lllllllll}\hat{w} & u & v & \theta_{2} & \beta_{1} & \beta_{2}, & \ldots & \beta_{n}\end{array}\right]^{T}$ was defined in Eq. (2.5.27), and the functions $\psi_{1}$ to $\psi_{m}$ are the same as in Eq. (2.3.13). 


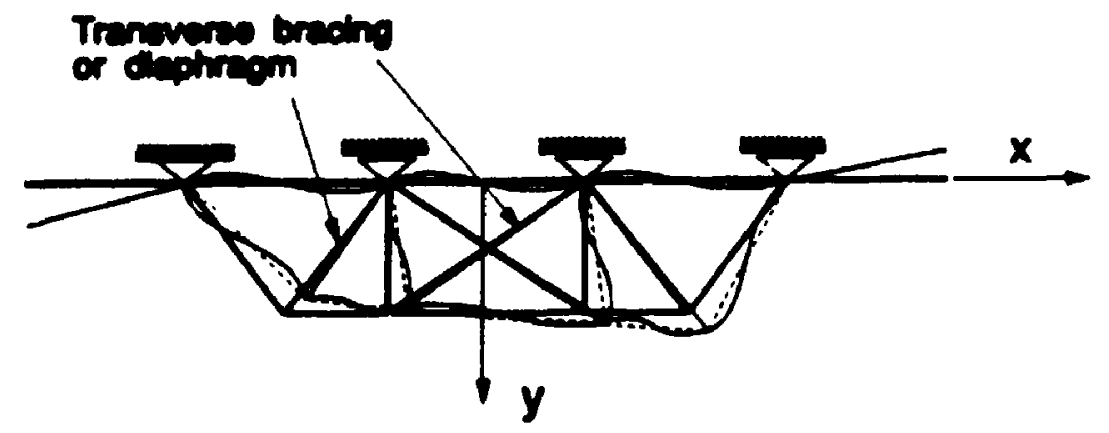

(a) Transeveres bending dus to distortional mode $\beta_{1}$

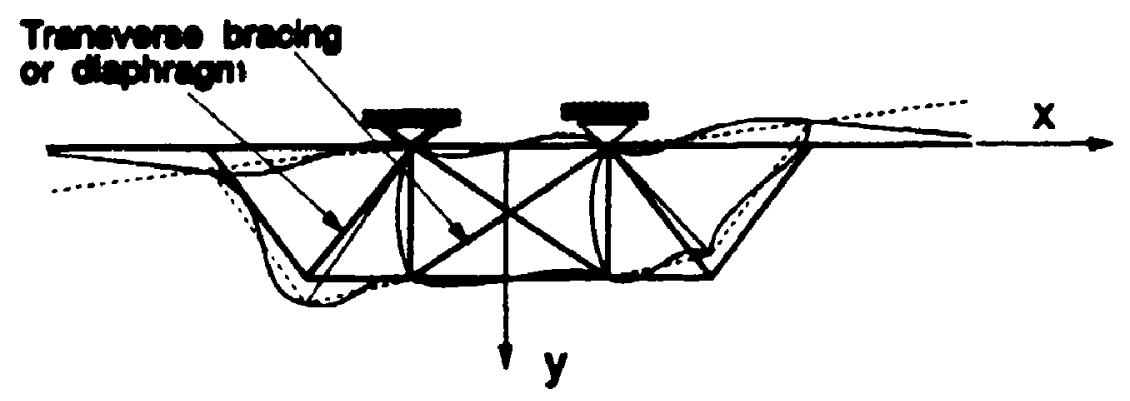

(b) Transverse bending due to distortional mode $\boldsymbol{\beta}_{2}$

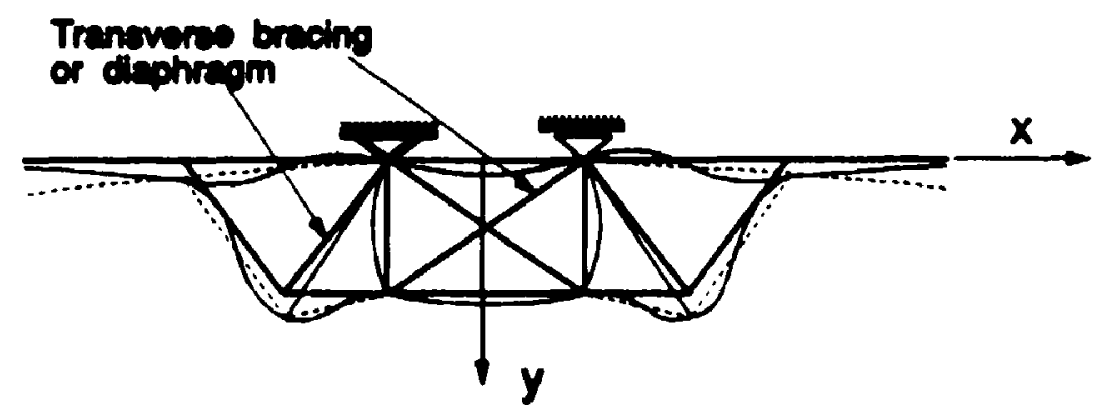

(c) Transverse bending due to distortional mode $\beta_{3}$

Fig. 2.18 Transverse bending due to different distortional modes.

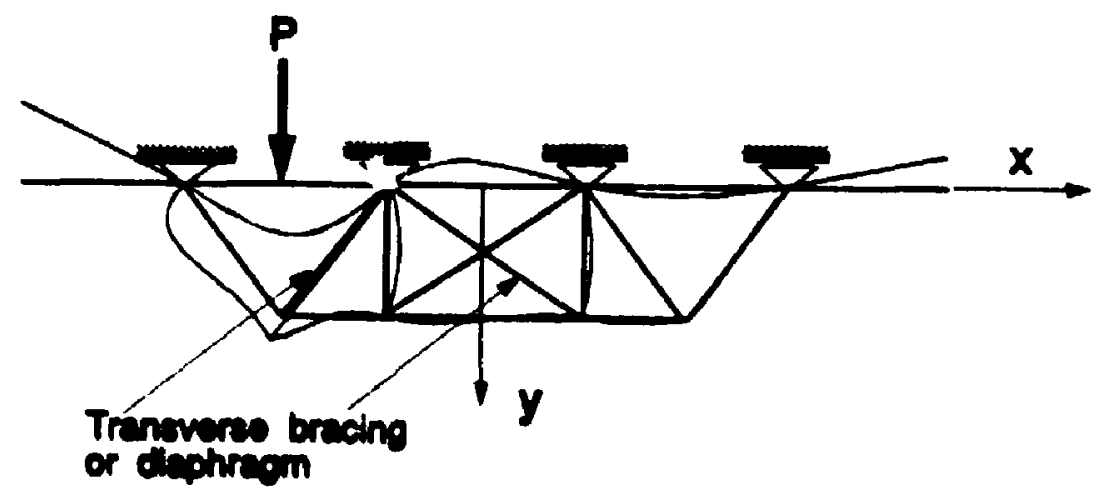

Fig. 2.19 Transverse bending due to loads applied between webs. 
The corresponding generalized warping vector, $\{\omega\}$, is

$$
\{\omega\}=\left\{\omega_{i}\right\}=\int_{0}^{s}(\{r\}-\{\tilde{r}\}) d s=\left\{\begin{array}{c}
1 \\
x \\
y \\
\omega \\
\omega_{d 1} \\
\cdot \\
\cdot \\
\cdot \\
\omega_{d n} \\
\omega_{v 1} \\
\cdot \\
\cdot \\
\cdot \\
\omega_{v m}
\end{array}\right\}
$$

and the vectors $\{r\}$ and $\{\bar{\tau}\}$ are

$$
\begin{aligned}
& \{r\}=\left[\begin{array}{llllllllllll}
0 & \cos \alpha & \sin \alpha & \rho & r_{1}, & \ldots & r_{n} & 0, & \ldots, & 0
\end{array}\right]^{T} \\
& \{\bar{\tau}\}=\left[\begin{array}{llllllllllll}
0 & 0 & 0 & \bar{\tau}_{s v} & \bar{\tau}_{1}, & \ldots & \bar{\tau}_{n} & \dot{\omega}_{v 1}, & \ldots & \dot{\omega}_{v m}
\end{array}\right]^{T}
\end{aligned}
$$

Using the principle of virtual work and procedures analogous to those in the last section, the governing differential equations of the problem can be written as

$$
[C]\left\{D^{I V}\right\}-[J]\left\{D^{\prime \prime}\right\}+[H]\{D\}=q_{x}\left\{r_{x}^{*}\right\}+q_{y}\left\{r_{y}^{*}\right\}+\int_{s} q_{z}^{\prime}\{\omega\} d s
$$

where

$$
\begin{gathered}
{[C]=\int_{A} E\{\omega\}\{\omega\}^{T} d A} \\
{[J]=\int_{A} G\{\bar{\tau}\}\{\bar{\tau}\}^{T} d A} \\
{[H]=\oint_{s} \frac{\left\{M_{s}(s)\right\}\left\{M_{s}(s)\right\}^{T}}{E_{s} I_{s}(s)} d s}
\end{gathered}
$$

in which

$$
\left\{M_{s}(s)\right\}=\left[\begin{array}{llllllllll}
0 & 0 & 0 & 0 & M_{s 1}(s), \ldots, M_{s n}(s) & 0 & \ldots, & 0
\end{array}\right]^{T}
$$

and $M_{\mathrm{si}}(s)$ is the bending moment distribution in the walls due to unit distortion $\beta_{1}$. 
Equation (2.6.5) contains the governing differential equations of multicell thinwalled box beams under axial, combined bending, torsional, distortional and shear lag effects. The system of simultanecus equations in Eq. (2.6.5) are coupled and can be uncoupled by a two step orthogonalization process. Where feasible, the exact solution of the uncoupled system of equations can be used to develop a thin-walled box beam finite element with exact shape functions. The next chapter will discuss the development of such a beam element. 


\section{STRAIGHT MULTICELL BEAM ELEMENT WITH EXACT SHAPE FUNCTIONS}

\subsection{General}

A straight box beam finite element is derived for modelling multicell box girder bridges. The proposed element is similar to a conventional beam element in the sense that only one element is needed to model a prismatic box girder between two supports.

The displacement formulation of the finite element method is adopted in this thesis. The fundamentals of the formulation are given briefly as the basis for later derivations. A two step orthogonalization procedure is described for uncoupling the governing differential equations of a box beam under extensional, flexural, torsional, distortional and shear lag effects. The exact solution of the governing equations is obtained and subsequently used to derive the exact shape functions for the proposed element. The stiffness matrix and equivalent nodal load vector of the element are explicitly derived based on the exact shape functions. The particular solution for each structural action mode corresponding to the most common loading conditions is presented. 


\subsection{Basics of Displacement Method of Finite Elements}

According to the displacement method of finite elements, the displacements $\{D\}$ at any point within the element are approximated by interpolation functions, called shape functions, in terms of the displacements at the nodes as follows:

$$
\{D\}=[N]\{D\}^{e}
$$

where $\{D\}$ is the element displacement vector at any point, $[N]$ is the shape function matrix and $\{D\}^{e}$ is the element nodal displacement vector.

Using the strain-displacement relations, the strain can be then determined as

$$
\{\epsilon\}=[B]\{D\}^{e}
$$

where $\{\epsilon\}$ is the strain vector at any point within the element and $[B]$ is the so-called strain-displacement matrix.

Stresses are related to strains through Hooke's law

$$
\{\sigma\}=[E]\{\epsilon\}
$$

where $\{\sigma\}$ is the stress vector at any point within the element and $[E]$ is the constitutive matrix. It should be pointed out that stresses and strains are in reality tensors and not vectors.

The equilibrium equation can be established by the principle of virtual work

$$
\sum_{\epsilon} \int_{V} \delta\{\epsilon\}^{T}\{\sigma] d V=\delta\{D\}^{e T}\{R\}+\sum_{e} \int_{V} \delta\{D\}^{T}\{q\} d V
$$

where $\{R\}$ is the vector of externally applied nodal loads, $\{q\}$ is the vector of applied distributed loads, $V$ is the volume of an element and $\sum$ is summation over all the elements, and the symbol $\delta$ represents virtual quantities.

Substituting Eqs. (3.2.2) and (3.2.3) into Eq. (3.3.4), the following can be found

$$
\sum_{e} \int_{V^{\circ}} \delta\{D\}^{e T}[B]^{T}[E][B]\{D\}^{e} d V=\delta\{D\}^{e T}\{R\}+\delta\{D\}^{e T} \sum_{e} \int_{V}[N]^{T}\{q\} d V
$$


or

$$
\delta\{D\}^{e T}\left(\sum_{e} \int_{V}[B]^{T}[E][B] d v\right)\{D\}^{e}=\delta\{D\}^{e T}\{R\}+\delta\{D\}^{e T} \sum_{e} \int_{V}[N]^{T}\{q\} d V
$$

Since $\delta\{D\}^{e}$ is arbitrary, Eq. (3.2.6) can be written as

$$
[S]\{D\}^{\prime}=\{R\}+\{F\}
$$

where $\{D\}^{\prime}$ is the nodal displacement vector of the structure which can be assembled from the element nodal displacement vector $\{D\}^{e}$ besed on the displacement continuity conditions, $[S]$ is the structural stiffness matrix and $\{F\}$ is the equivalent nodal load vector of the structure, which are given by

$$
\begin{gathered}
{[S]=\sum_{e}[S]^{e}} \\
\{F\}=\sum_{e}\{F\}^{e}
\end{gathered}
$$

in which $[S]^{e}$ is the element stiffness matrix and $\{F\}^{e}$ is the equivalent element nodal load vector, i.e.

$$
\begin{gathered}
{[S\}^{e}=\int_{V}[B]^{T}[E][B] d V} \\
\{F\}^{e}=\int_{V}[N]^{T}\{q\} d V
\end{gathered}
$$

The nodal displacements $\{D\}^{\text {s }}$ of the structure can be found by solving Eq. (3.2.7) while strains and stresses can be obtained from Eqs. (3.2.2) and (3.2.3), respectively.

\subsection{Uncoupling the Governing Equations}

In this section, a two step orthogonalization procedure is presented to uncouple the governing differential equations. The method is similar to that of determining the principal coordinate axes of the cross section in flexural analysis or the shear center in torsional warping analysis. In the first step, the distortional and shear lag modes are uncoupled from the axial, bending and torsion. In the second step, an eigenvalue procedure will be used to uncouple the distortional and shear lag modes. 


\subsubsection{Uncoupling the Distortional and Shear Lag Modes from the Non-Distortional Modes}

In this step we uncouple the distortional and shear lag effects from the axial, bending and torsional effects. This means that elements $C_{i j}$ ( $i=5, \bar{m} ; j=1,4, \bar{n}=$ $4+n$ and $\bar{m}=\bar{n}+m$ ) of matrix $[C]$ are rendered zero. The desired transformation may be achieved by defining a matrix $\left[T_{a}\right]$ as follows:

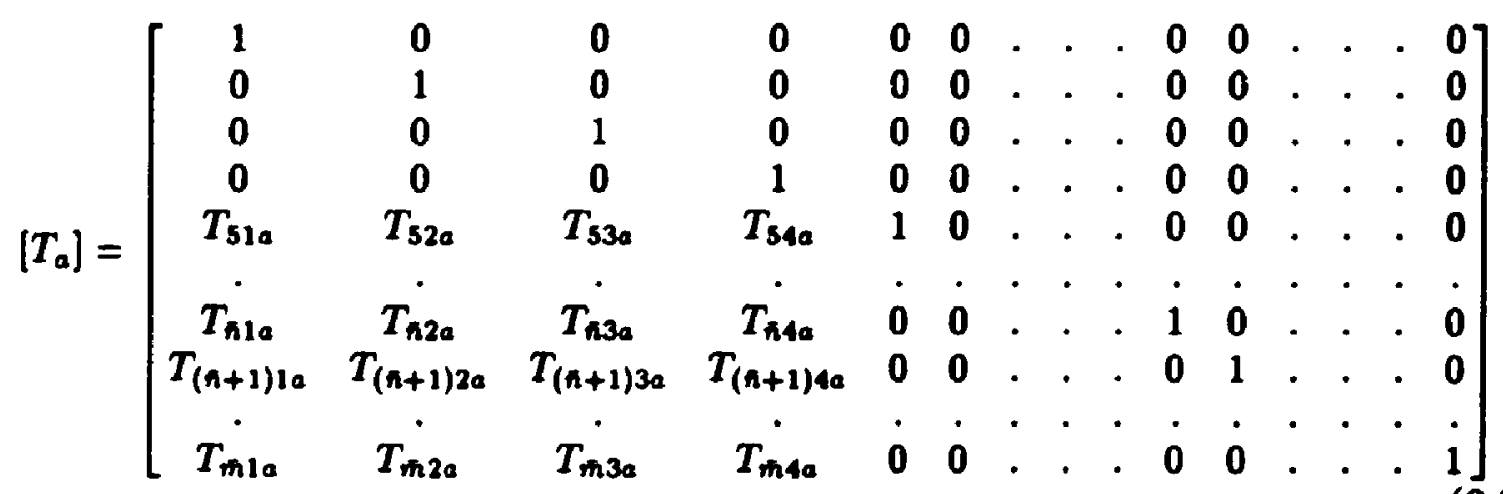

The unknown elements of $\left[T_{a}\right]$ can be obtained from the relations

$$
\begin{gathered}
{\left[\begin{array}{c}
C_{5 j} \\
C_{6 j} \\
\cdot \\
\cdot \\
C_{n j}
\end{array}\right]+C_{j j}\left[\begin{array}{c}
T_{5 j a} \\
T_{6 j a} \\
\cdot \\
\cdot \\
\cdot \\
T_{n j a}
\end{array}\right]=0 \quad(j=1,2,3)} \\
{\left[\begin{array}{c}
C_{54} \\
C_{64} \\
\cdot \\
\cdot \\
\dot{C_{n 4}}
\end{array}\right]+J_{44}\left[\begin{array}{c}
T_{54 a} \\
T_{64 a} \\
\cdot \\
\cdot \\
\cdot \\
T_{n 4 a}
\end{array}\right]=0}
\end{gathered}
$$

and

$$
\left[\begin{array}{c}
C_{(n+1) \jmath} \\
C_{(n+2) \jmath} \\
\cdot \\
\cdot \\
C_{m j}
\end{array}\right]+C_{J}\left[\begin{array}{c}
T_{(n+1) \jmath a} \\
T_{(n+2), a} \\
\cdot \\
\cdot \\
\cdot \\
T_{m j a}
\end{array}\right]=0, \quad(j=1,2,3,4)
$$

After this first coordinate transformation, matrices $[C],[J]$ and $[H]$ reduce to 
the following form, where $Y$ denotes a non-zero element.

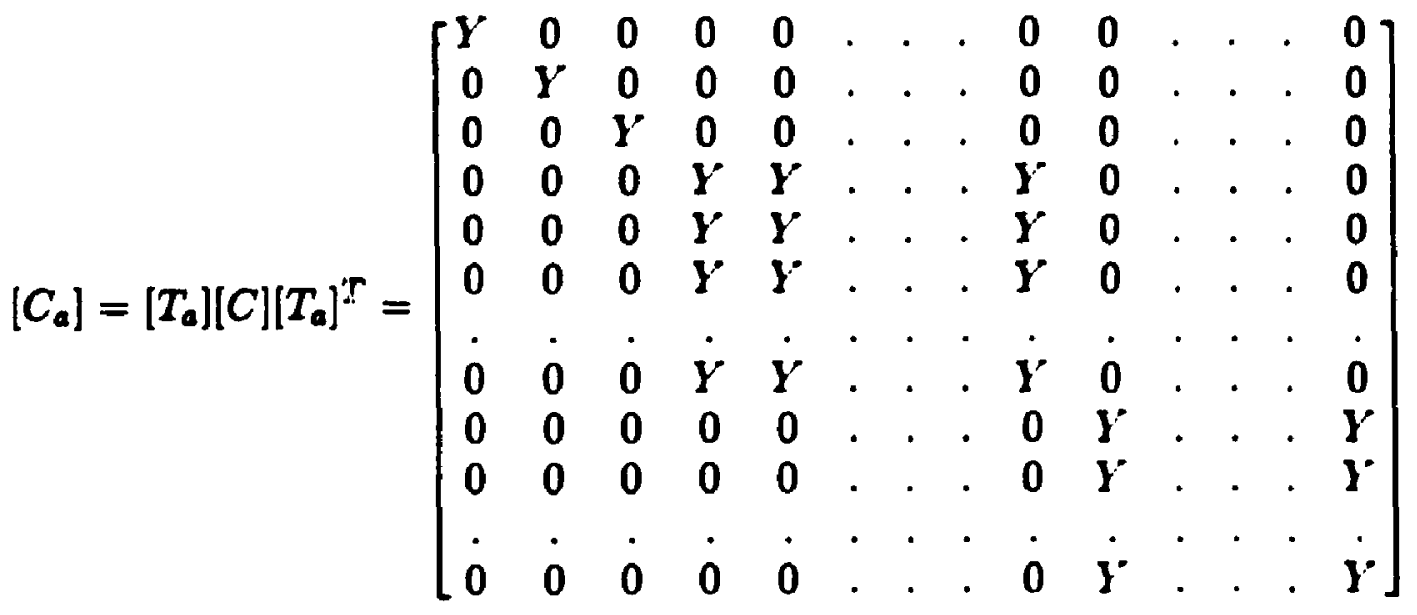

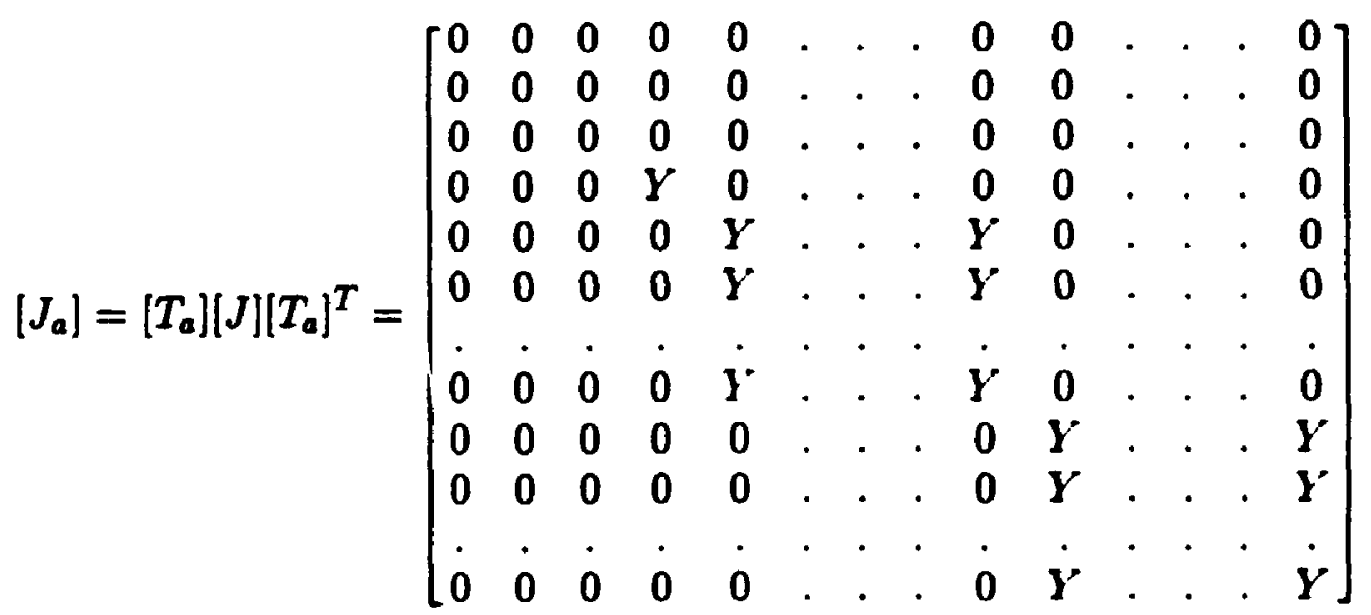

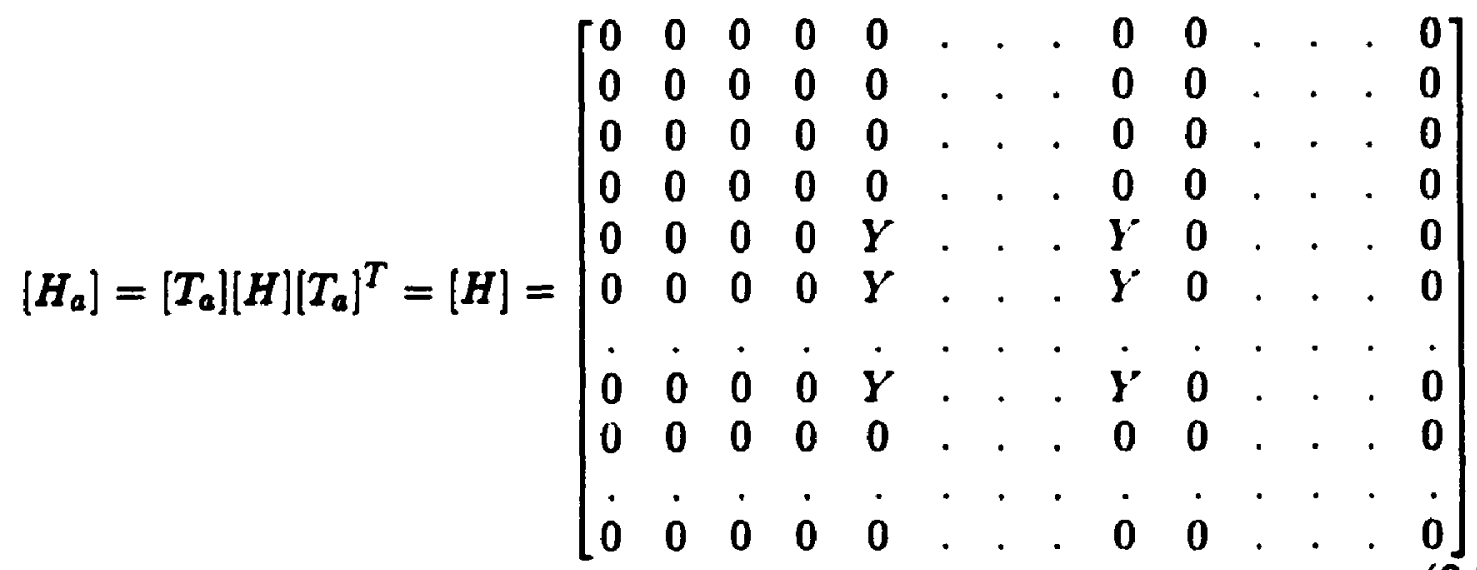

Any non-zero warping stiffness among the terms $C_{54 a}, C_{64 a}, \ldots, C_{n 4 a}$ is neglected as being of relatively low structural significance in a closed box beam (Sedlacek 1968). 


\subsubsection{Uncoupling the Distortional and Shear Lag Modes}

After the first step orthogonalization, it can be seen in Eqs. (3.3.5), (3.3.6) and (3.3.7) that the non-distortional modes are uncoupled from the distortional and shear lag modes, but the distortional modes are still coupled among each other as well as with the shear lag modes. Sedlacek (1968) used a standard eigenvalue procedure to transform $\left[C_{a}\right],\left[J_{a}\right]$ and $\left[H_{a}\right]$ into diagonalized matrices. The off-diagonal terms of $\left[J_{a}\right]$ for distortional modes were neglected because they were found to be small.

For distortional modes, the transformation matrix $\left[T_{b}\right]$ is the matrix of eigenvectors of the following eigenvalue problem:

$$
\left(\left[C_{a}\right]-\lambda\left[H_{a}\right]\right)\{D\}=0
$$

For shear lag modes, the transformation matrix $\left[T_{c}\right]$ is the matrix of eignvectors of the eignvalue problem

$$
\left(\left[C_{a}\right]-\lambda\left[J_{a}\right]\right)\{D\}=0
$$

After the preceding ortiogonalizations, Eq. (2.6.10) becomes

$$
[\bar{C}]\{\bar{D}\}^{I V}-[\bar{J}]\{\bar{D}\}^{\prime \prime}+[\bar{H}]\{\bar{D}\}=q_{y}\left\{\bar{r}_{y}^{*}\right\}+q_{x}\left\{\bar{r}_{z}^{*}\right\}+\int_{s} q_{x}^{\prime}\{\bar{w}\} d s
$$

where

$$
[T]=\left[T_{c}\right]\left[T_{b}\right]\left[T_{a}\right]
$$

The vectors $\left\{\bar{u}^{\prime}\right\},\{\bar{D}\},\left\{\bar{r}_{y}^{*}\right\}$ and $\left\{\bar{r}_{z}^{*}\right\}$, and the matrices $[\bar{C}],[\bar{J}]$ and $[\bar{H}]$ are the same as the corresponding quantities defined earlier, but are now expressed in the orthogonalized coordinate system. For example,

$$
\begin{gathered}
\{\bar{D}\}=[T]\{D\} \\
{[\bar{C}]=[T][C][T]^{T}}
\end{gathered}
$$

After the transformation, $[\bar{C}],[\bar{J}]$ and $[\bar{H}]$ become diagonal matrices of the following 
forms:

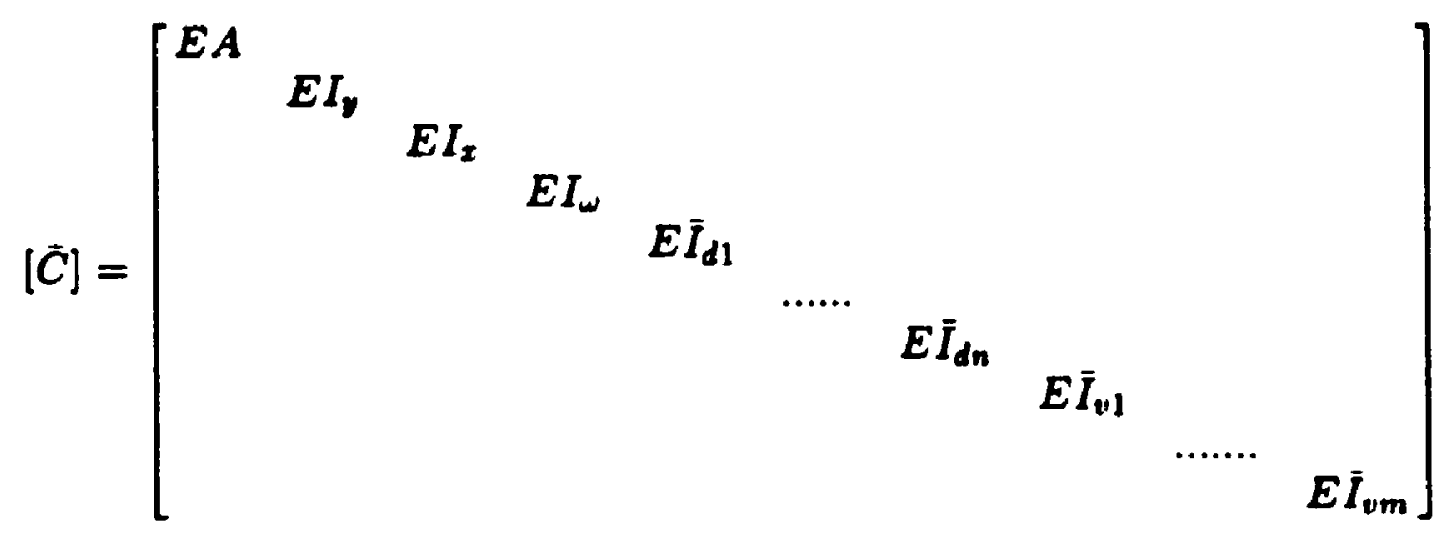

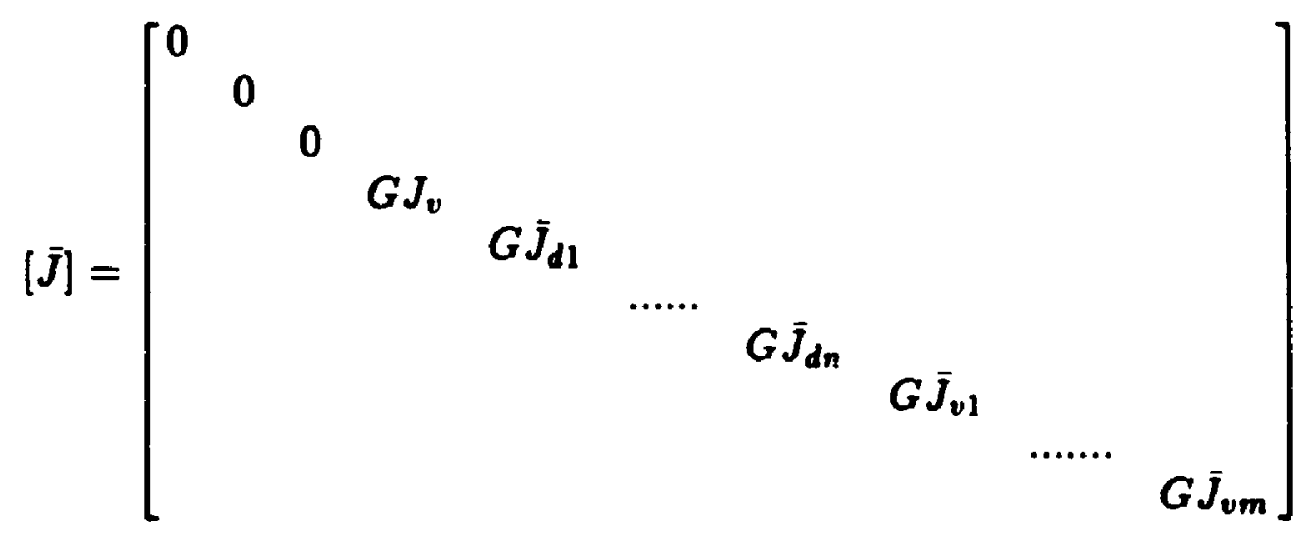

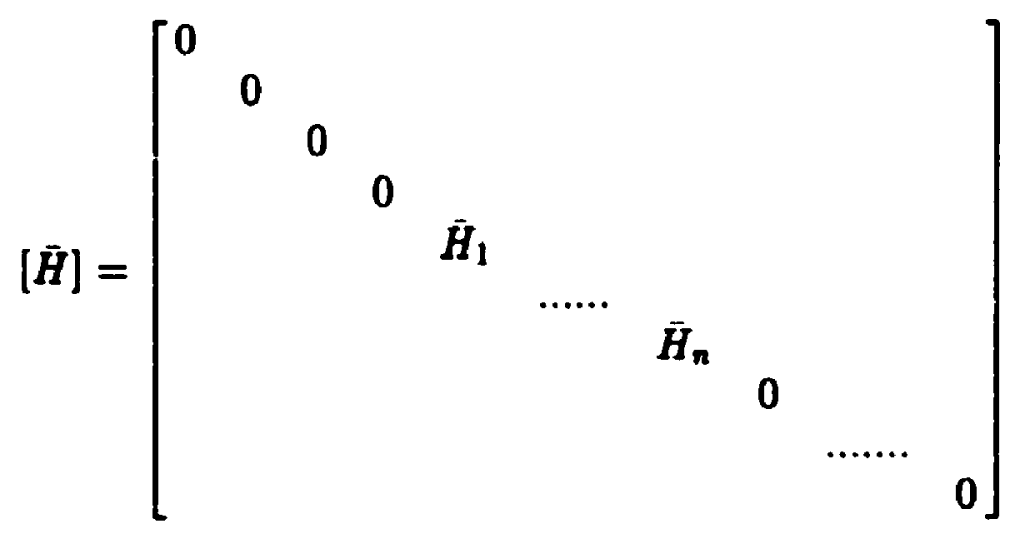

It should be observed that elements of matrices $[\bar{C}]$ and $[\bar{J}]$ are the box rigidities corresponding to the various structural actions. In the computer program developed based on the procedures described in this thesis, the required rigidities are calculated in the program from the input values of the elastic and shear moduli and the dimensions of the box girder cross-section (see Appendix A). 


\subsection{Exact Solution of Uncoupled Governing Equations}

The uncoupled differential equations governing the axial, flexural, torsional, distortional warping and shear lag effects in thin-walled multicell box girders can be rewritten, respectively, as:

$$
\begin{gathered}
E A \frac{d^{2} w}{d z^{2}}=q_{z} \\
E I_{y} \frac{d^{4} u}{d z^{4}}=q_{x} \\
E I_{x} \frac{d^{4} v}{d z^{4}}=q_{y} \\
E I_{w} \frac{d^{4} \theta_{z}}{d z^{4}}-G J_{v} \frac{d^{2} \theta_{z}}{d z^{2}}=m_{z} \\
E \bar{I}_{v i} \frac{d^{4} \bar{\psi}_{i}}{d z^{4}}-G \bar{J}_{v i} \frac{d^{2} \bar{\psi}_{i}}{d z^{2}}=\bar{q}_{v i} \\
E \bar{I}_{d i} \frac{d^{4} \bar{\beta}_{i}}{d z^{4}}-G \bar{J}_{d i} \frac{d^{2} \bar{\beta}_{i}}{d z^{2}}+\bar{H}_{i} \bar{\beta}_{i}=\bar{q}_{d i}
\end{gathered}
$$

where $\bar{q}_{v i}$ is the generalized load corresponding to the shear lag mode $v i$ which is generated by the orthogonalization flexure and shear lag, and $\bar{q}_{d i}$ is the generalized load corresponding to the distortional mode $i$ which is due to the combined effects of torsional moment $m_{z}$ and the orthogonalization process.

The complete solution of these equations is given by the solution of the homogeneous part plus the particular integral corresponding to $q(z)$. In deriving the stiffness matrix, it is the homogeneous solution that is of interest, and the particular solution corresponding to different loading conditions is needed to determine the displacements and stresses within the span, which will be discussed in the next section.

\subsubsection{Extension}

The solution of the homogeneous part of Eq. (3.4.1a) for extension is given by

$$
u=\left[\begin{array}{ll}
(1-\bar{z}) & \bar{z}
\end{array}\right]\{d\}
$$


where $\bar{z}=z / l$ and $\{d\}=\left[(w)_{z=0}(w)_{z=1}\right]^{T}$. The alternative form of Eq. (3.4.2), as in the finite element method, can be written as

$$
u=\left[N_{1}\right]\{d\}
$$

where $\left[N_{1}\right]$ is the shape function matrix for extension and is equal to

$$
\left[N_{1}\right]=\left[\begin{array}{ll}
N_{1} & N_{2}
\end{array}\right]=\left[\begin{array}{ll}
(1-\bar{z}) & \bar{z}
\end{array}\right]
$$

\subsubsection{Flexure}

The solution of the homogeneous part of Eqs. (3.4.1b) and (3.4.1c) for flexure is given by

$$
u=\left[N_{j}\right]\{d\}
$$

where $\left[N_{j}\right]$ is the shape function matrix for flexure with $j=2$ for $x$ direction bending and $j=3$ for $y$ direction bending. The shape function matrix is given by

$$
\left[N_{2}\right]=\left[N_{3}\right]=\left[\begin{array}{lll}
\left(1-3 \bar{z}^{2}+2 \bar{z}^{3}\right) & \bar{z} l\left(1-2 \bar{z}+\bar{z}^{2}\right) & \left(3 \bar{z}^{2}-2 \bar{z}^{3}\right) \quad \bar{z} l\left(\bar{z}^{2}-\bar{z}\right)
\end{array}\right]
$$

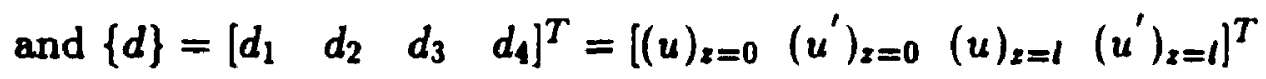

\subsubsection{Torsion and Shear Lag}

It can be seen that Eqs. (3.4.1d; and (3.4.1e) have the same form as the governing differential equation of an elastic beam subjected to a constant tensile force at its ends as indicated by Fig. 3.1, and they can be written in a general form as

$$
E I \frac{d^{4} y}{d z^{4}}-\mathcal{N} \frac{d^{2} y}{d z^{2}}=0
$$

where

$$
\begin{aligned}
& y=\theta_{z} \\
& E I=E I_{\omega} \\
& \mathcal{N}=G J_{v}
\end{aligned}
$$

for torsion, and

$$
\begin{aligned}
& y=\bar{\psi}_{i} \\
& E I=E \bar{I}_{v i}
\end{aligned}
$$




$$
\mathcal{N}=G \bar{J}_{v}
$$

for the shear lag mode $i$.
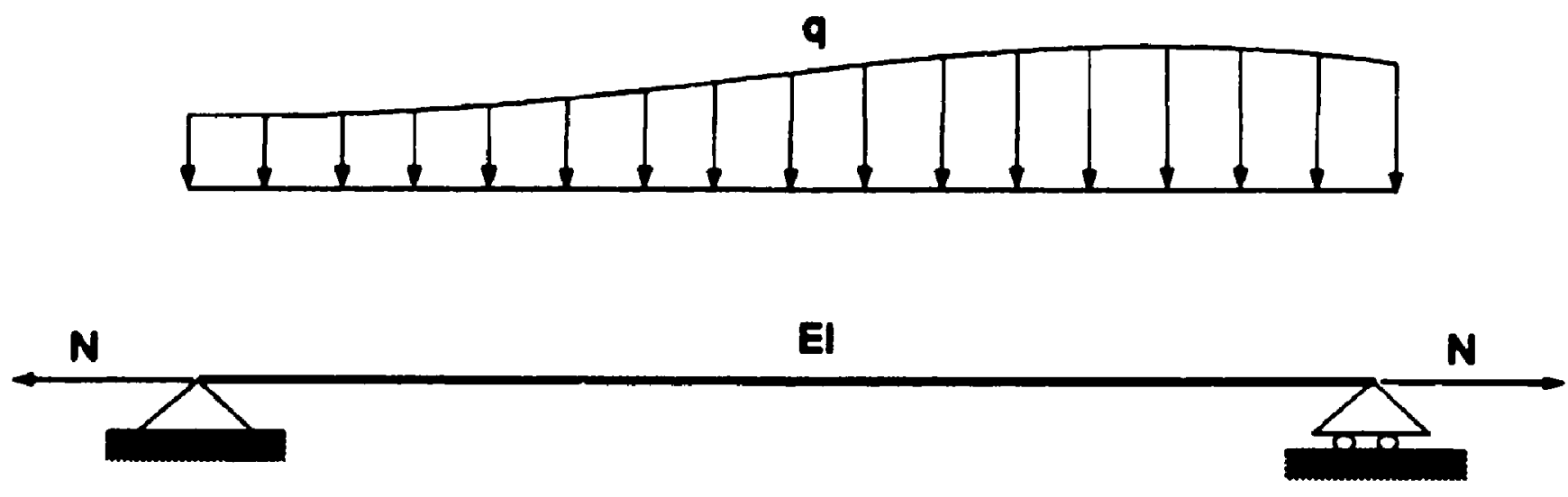

Fig. 3.1 Beam-column analogy for torsional warping and shear lag.

The solution of Eq. (3.4.4) may be written as

$$
y=A_{1} e^{\zeta z}+A_{2} e^{-\zeta 2}+A_{3} z+A_{4}=\left[\begin{array}{llll}
e^{\zeta z} & e^{-\zeta z} & z & 1
\end{array}\right]\{A\}
$$

or

$$
y=[L]\{A\}
$$

where $\zeta=\sqrt{\frac{N}{E T}}$. The constants $A_{1}$ to $A_{4}$ can be solved for in terms of $y$ and its first derivative, $y^{\prime}$, at the two ends or nodes of the element. Thus

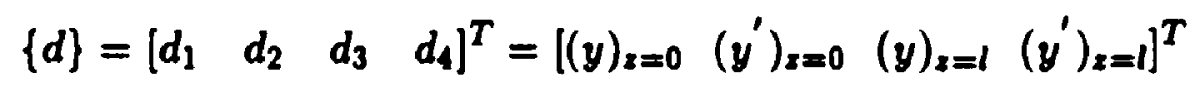

Substituting the boundary conditions from Eq. (3.4.9) into Eq. (3.4.8b), we can write

$$
y=[L][Q]\{d\}=\left[N_{i}\right]\{d\}
$$

where

or

$$
[Q]=\left[\begin{array}{cccc}
1 & 1 & 0 & 1 \\
\zeta & -\zeta & 1 & 0 \\
e^{\zeta 1} & e^{-\zeta l} & 1 & 1 \\
\zeta e^{\zeta l} & -\zeta e^{-\zeta 1} & 1 & 0
\end{array}\right]^{-1}
$$

$$
[Q]=\left[\begin{array}{llll}
Q_{11} & Q_{12} & Q_{13} & Q_{11} \\
Q_{21} & Q_{22} & Q_{23} & Q_{24} \\
Q_{31} & Q_{32} & Q_{33} & Q_{34} \\
Q_{41} & Q_{12} & Q_{43} & Q_{44}
\end{array}\right]
$$


in which

$$
\begin{aligned}
& Q_{11}=-Q_{13}=\frac{1}{2-2 e^{16}+1 \zeta+e^{1 \zeta / \zeta}} \\
& Q_{12}=\frac{-1+e^{16}-15}{\left(-1+e^{16}\right) 6\left(2-2 e^{16}+16+e^{1616}\right)} \\
& Q_{14}=\frac{e^{1 \zeta}\left(-1+e^{-(1 \zeta)}+1 \zeta\right)}{\left(-1+e^{1 \zeta}\right) \zeta\left(2-2 e^{16}+1 \zeta+e^{1 \zeta / \zeta}\right)} \\
& Q_{21}=-Q_{23}=-\frac{e^{1 \zeta}}{2-2 e^{k}+1 \zeta+e^{k \zeta / \zeta}} \\
& Q_{22}=\frac{e^{16}\left(-1+e^{16}-e^{16 / \zeta}\right)}{\left(-1+e^{16}\right) \zeta\left(2-2 e^{16}+16+e^{16 / \zeta}\right)} \\
& Q_{24}=\frac{e^{16}\left(1-e^{16}+1 \zeta\right)}{\left(-1+e^{16}\right) \zeta\left(2-2 e^{16}+16+e^{16 / \zeta}\right)} \\
& Q_{31}=-Q_{33}=-\frac{\left(1+e^{l \zeta}\right) \zeta}{2-2 e^{1 \zeta}+1 \zeta+e^{\mid \zeta / \zeta}} \\
& Q_{32}=Q_{34}=Q_{13}=-\frac{-1+e^{1 \zeta}}{2-2 e^{1 \zeta}+l \zeta+e^{\zeta / \zeta}} \\
& Q_{41}=\frac{1-e^{k \zeta}+1 \zeta+e^{l \zeta} / \zeta}{2-2 e^{k \zeta}+1 \zeta+e^{1 \zeta 1 \zeta}} \\
& Q_{42}=\frac{1-e^{21 \zeta}+1 \zeta+e^{21 \zeta} l \zeta}{\left(-1+e^{1 \zeta}\right) \zeta\left(2-2 e^{1 \zeta}+1 \zeta+e^{1 \zeta} / \zeta\right)} \\
& Q_{44}=\frac{-1+e^{21 \zeta}-2 e^{1 \zeta} l \zeta}{\left(-1+e^{1 \zeta}\right) \zeta\left(2-2 e^{1 \zeta}+l \zeta+e^{1 \zeta l \zeta}\right)}
\end{aligned}
$$

It may be noted that $\left[N_{i}\right]$ is the shape function matrix for torsion and shear lag modes with $i=4$ for torsion and $i=v i$ for shear lag mode vi. A typical shape function for torsion and shear lag corresponding to $d_{1}=1, d_{2}=d_{3}=d_{4}=0$, is given by

$$
N_{1}=Q_{11} e^{\sigma_{2}}+Q_{21} e^{-\zeta_{2}}+Q_{31} z+Q_{41}
$$

This is in contrast to the corresponding shape function of a conventional beam, i.e. a beam without axial force which is given by Eq. (3.4.6).

\subsubsection{Distortion}

The solution of the homogeneous part of beam-column on elastic foundation Eq. 
(3.4.1f). as shown in Fig. 3.2, i.e.

$$
E \bar{I}_{d i} \frac{d^{4} \bar{\beta}_{1}}{d z^{4}}-G \bar{J}_{d i} \frac{d^{2} \bar{\beta}_{i}}{d z^{2}}+\bar{H}_{i} \bar{\beta}_{i}=0
$$

will depend on the relative magnitude of the coefficients of the above equation. Razaqpur and Shah (1990) have provided the complete solution of this equation using the matrix formulation. Thus, for $G \bar{J}_{d i}>\sqrt{4 \bar{H}_{i} E \bar{I}_{d i}}$, referred to henceforth as case I,

$\bar{\beta}_{i}=A_{1} \cosh \lambda_{1} z \cosh \gamma z+A_{2} \cosh \lambda_{1} z \sinh \gamma z+A_{3} \sinh \lambda_{1} z \cosh \gamma z+A_{4} \sinh \lambda_{1} z \sinh \gamma z$

where

$$
\begin{aligned}
& \lambda_{1}=\sqrt{\frac{G \bar{J}_{d i}}{4 E \bar{I}_{d i}}+\sqrt{\frac{\bar{H}_{i}}{4 E \bar{I}_{d i}}}} \\
& \gamma=\sqrt{\frac{G \bar{J}_{d i}}{4 E \bar{I}_{d i}}-\sqrt{\frac{\bar{H}_{i}}{4 E \bar{I}_{d i}}}}
\end{aligned}
$$
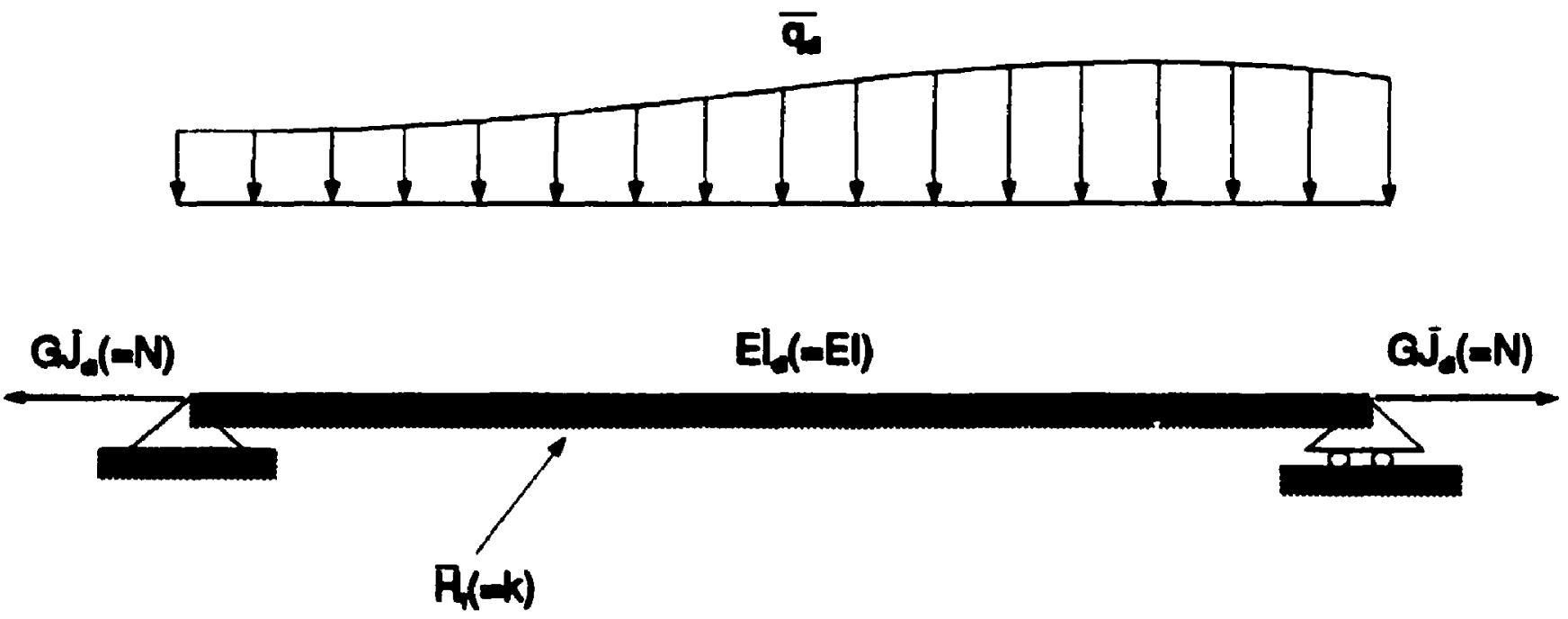

Fig. 3.2 Beam-column-on-elastic-foundation analogy for distortion. For $G \bar{J}_{d_{1}} \leq \sqrt{4 \bar{H}_{i} E \bar{I}_{d_{i}}}$ or case II, $\bar{\beta}_{1}=A_{1} \cosh \lambda_{1} z \cos \gamma_{1} z+A_{2} \cosh \lambda_{1} z \sin \gamma_{1} z+A_{3} \sinh \lambda_{1} z \cos \gamma_{1} z+A_{4} \sinh \lambda_{1} z \sin \gamma_{1} z$ 
where

$$
\gamma_{1}=\sqrt{\sqrt{\frac{\bar{H}_{1}}{4 E \bar{I}_{d i}}}-\frac{G \bar{J}_{d i}}{4 E \bar{I}_{d i}}}
$$

Eqs. (3.4.15) and (3.4.18) can be solved similar to Eq. (3.4.6a) by using the boundary conditions:

$$
\{d\}=\left[\begin{array}{llll}
\left(\bar{\beta}_{1}\right)_{z=0} & \left(\bar{\beta}_{1}^{\prime}\right)_{z=0} & \left(\bar{\beta}_{1}\right)_{z=1} \quad\left(\bar{\beta}_{1}^{\prime}\right)_{z=1}{ }^{T}
\end{array}\right.
$$

This will lead for case I to

$$
\bar{\beta}_{i}=\left[\cosh \lambda_{1} z \cosh \gamma z \cosh \lambda_{1} z \sinh \gamma z \sinh \lambda_{1} z \cosh \gamma z \sinh \lambda_{1} z \sinh \gamma z\right][Q 1]\{d\}
$$

or

$$
\bar{\beta}_{i}=\left[N_{d_{1}}\right]\{d\}
$$

where $\left[N_{d i}\right]$ is the shape function matrix for distortion for case $I$ and is given by

$$
\left[N_{a i}\right]=\left[\begin{array}{llll}
\cosh \lambda_{1} z \cosh \gamma z & \cosh \lambda_{1} z \sinh \gamma z \sinh \lambda_{1} z \cosh \gamma z & \sinh \lambda_{1} z \sinh \gamma z
\end{array}\right][Q 1]
$$

in which

$$
[Q 1]=\left[\begin{array}{cccc}
1 & 0 & 0 & 0 \\
0 & \gamma & \lambda_{1} & 0 \\
c_{1} c_{2} & c_{1} s_{2} & c_{2} s_{1} & s_{1} s_{2} \\
c_{2} \lambda_{1} s_{1}+c_{1} \gamma s_{2} & c_{1} c_{2} \gamma+\lambda_{1} s_{1} s_{2} & c_{1} c_{2} \lambda_{1}+\gamma s_{1} s_{2} & c_{2} \gamma s_{1}+c_{1} \lambda_{1} s_{2}
\end{array}\right]^{-1}
$$

or

$$
[Q 1]=\left[\begin{array}{llll}
Q 1_{11} & Q 1_{12} & Q 1_{13} & Q 1_{14} \\
Q 1_{21} & Q 1_{22} & Q 1_{23} & Q 1_{24} \\
Q 1_{31} & Q 1_{32} & Q 1_{33} & Q 1_{34} \\
Q 1_{41} & Q 1_{12} & Q 1_{13} & Q 1_{44}
\end{array}\right]
$$

where

$$
\begin{aligned}
& Q 1_{11}=1 \\
& Q 1_{12}=Q 1_{13}=Q 1_{14}=0 \\
& Q 1_{21}=\frac{\lambda_{1}}{\gamma}\left(\frac{\gamma c_{1} s_{1}+\lambda_{1} c_{2} s_{2}}{\Delta}\right) \\
& Q 1_{22}=\frac{s_{1}^{2}}{\Delta} \\
& Q 1_{23}=-\frac{\lambda_{1}}{\gamma}\left(\frac{\gamma c_{2} s_{1}}{\Delta}+\frac{\lambda_{1} c_{1} s_{2}}{\Delta}\right)
\end{aligned}
$$




$$
\begin{aligned}
& Q 1_{24}=\frac{\lambda_{1}}{\gamma}\left(\frac{s_{2} s_{1}}{\Delta}\right) \\
& Q 1_{31}=-\left(\frac{\gamma c_{1} s_{1}+\lambda_{1} c_{2} s_{2}}{\Delta}\right) \\
& Q 1_{32}=\frac{\lambda_{1}}{\gamma}\left(\frac{s_{2}^{2}}{\Delta}\right) \\
& Q 1_{33}=\left(\frac{\gamma c_{2} s_{1}+\lambda_{1} c_{1} s_{2}}{\Delta}\right) \\
& Q 1_{34}=-\frac{s_{2} s_{1}}{\Delta} \\
& Q 1_{41}=\lambda_{1}\left(\frac{s_{2}^{2} c_{1}^{2}-c_{2}^{2} s_{1}^{2}}{\Delta}\right) \\
& Q 1_{42}=\frac{1}{\gamma}\left(\frac{\lambda_{1} c_{2} s_{2}-\gamma c_{1} s_{1}}{\Delta}\right) \\
& Q 1_{43}=\frac{\lambda_{1}^{2}-\gamma^{2}}{\gamma}\left(\frac{s_{2} s_{1}}{\Delta}\right) \\
& Q 1_{14}=\frac{1}{\gamma}\left(\frac{\gamma c_{2} s_{1}-\lambda_{1} s_{2} c_{1}}{\Delta}\right)
\end{aligned}
$$

in which $c_{1}=\cosh \lambda_{1} l, c_{2}=\cosh \gamma l, s_{1}=\sinh \lambda_{1} l, s_{2}=\sinh \gamma l$ and

$$
\Delta=\left(\frac{\gamma^{2} s_{1}^{2}-\lambda_{1}^{2} s_{2}^{2}}{\gamma}\right)
$$

and for case II to

$$
\bar{\beta}_{1}=\left[\cosh \lambda_{1} z \cos \gamma_{1} z \cosh \lambda_{1} z \sin \gamma_{1} z \sinh \lambda_{1} z \cos \gamma_{1} z \sinh \lambda_{1} z \sin \gamma_{1} z\right][Q 2]\{d\}
$$

or

$$
\bar{\beta}_{\imath}=\left\{N_{d l}\right]\{d\}
$$

where $\left[\lambda_{d_{1}}\right]$ is the shape function matrix for case II and is given by

$$
\left[N_{\mathrm{d}_{1}}\right]=\left[\cosh \lambda_{1} z \cos \gamma_{1} z \cosh \lambda_{1} z \sin \gamma_{1} z \sinh \lambda_{1} z \cos \gamma_{1} z \sinh \lambda_{1} z \sin \gamma_{1} z\right]\left[Q_{2}\right]
$$

in which

$$
[Q 2]=\left[\begin{array}{cccc}
1 & 0 & 0 & 0 \\
0 & \gamma_{1} & \lambda_{1} & 0 \\
c_{1} c_{3} & c_{1} s_{3} & c_{3} s_{1} & s_{1} s_{3} \\
c_{3} \lambda_{1} s_{1}-c_{1} s_{1} s_{3} & c_{1} c_{3} \gamma_{1}+\lambda_{1} s_{1} s_{3} & c_{1} c_{3} \lambda_{1}-\gamma_{1} s_{1} s_{3} & c_{3} \gamma_{1} s_{1}+c_{1} \lambda_{1} s_{3}
\end{array}\right]^{-1}
$$


or

$$
[Q 2]=\left[\begin{array}{llll}
Q 2_{11} & Q 2_{12} & Q 2_{13} & Q 2_{14} \\
Q 2_{21} & Q 2_{22} & Q 2_{23} & Q 2_{24} \\
Q 2_{31} & Q 2_{32} & Q 2_{33} & Q 2_{34} \\
Q 2_{41} & Q 2_{42} & Q 2_{43} & Q 2_{44}
\end{array}\right]
$$

where

$$
\begin{aligned}
& Q_{11}=1 \\
& Q 2_{12}=Q 2_{13}=Q 2_{14}=0 \\
& Q 2_{21}=\frac{\lambda_{1}}{\gamma_{1}}\left(\frac{\gamma_{1} c_{1} s_{1}+\lambda_{1} c_{3} s_{3}}{\Delta_{1}}\right) \\
& Q 2_{22}=\frac{s_{1}^{2}}{\Delta_{1}} \\
& Q 223=-\frac{\lambda_{1}}{\gamma_{1}}\left(\frac{\gamma_{1} c_{3} s_{1}+\lambda_{1} c_{1} s_{3}}{\Delta_{1}}\right) \\
& Q 2_{24}=\frac{\lambda_{1}}{\gamma_{1}}\left(\frac{s_{3} s_{1}}{\Delta_{1}}\right) \\
& Q 2_{31}=-\left(\frac{\gamma_{1} c_{1} s_{1}+\lambda_{1} c_{3} s_{3}}{\Delta_{1}}\right) \\
& Q 2_{32}=\frac{\lambda_{1}}{\gamma_{1}}\left(\frac{s_{3}^{2}}{\Delta_{1}}\right) \\
& Q 2_{33}=\left(\frac{\gamma_{1} c_{3} s_{1}+\lambda_{1} c_{1} s_{3}}{\Delta_{1}}\right) \\
& Q 2_{34}=-\frac{s_{3} s_{1}}{\Delta_{1}} \\
& Q 2_{41}=\lambda_{1}\left(\frac{s_{3}^{2} c_{1}^{2}-c_{3}^{2} s_{1}^{2}}{\Delta_{1}}\right) \\
& Q 2_{42}=\frac{1}{\gamma_{1}}\left(\frac{\lambda_{1} c_{3} s_{3}-\gamma_{1} c_{1} s_{1}}{\Delta_{1}}\right) \\
& Q 2_{43}=\frac{\lambda_{1}^{2}-\gamma_{1}^{2}}{\gamma_{1}}\left(\frac{s_{3} s_{1}}{\Delta_{1}}\right) \\
& Q 2_{14}=\frac{1}{\gamma_{1}}\left(\frac{\gamma_{1} c_{3} s_{1}-\lambda_{1} s_{3} c_{1}}{\Delta_{1}}\right)
\end{aligned}
$$

in which $c_{3}=\cos \gamma_{1} l . s_{3}=\sin \gamma_{1} l$ and

$$
\Delta_{1}=\left(\frac{\gamma_{1}^{2} s_{1}^{2}-\lambda_{1}^{2} s_{3}^{2}}{\gamma_{1}}\right)
$$

A typical shape function for distortion corresponding to $d_{1}=1, d_{2}=d_{3}=d_{4}=0$. is given by

$$
\begin{aligned}
N_{1}= & Q 1_{11} \cosh \lambda_{1} z \cosh \gamma z+Q 1_{21} \cosh \lambda_{1} z \sinh \gamma z+ \\
& Q 1_{31} \sinh \lambda_{1} z \cosh \gamma z+Q 1_{11} \sinh \lambda_{1} z \sinh \gamma z
\end{aligned}
$$


for case I, and

$$
\begin{aligned}
N_{1}= & Q 2_{11} \cosh \lambda_{1} z \cos \gamma_{1} z+Q 2_{21} \cosh \lambda_{1} z \sin \gamma_{1} z+ \\
& Q 2_{31} \sinh \lambda_{1} z \cos \gamma_{1} z+Q 2_{41} \sinh \lambda_{1} z \sin \gamma_{1} z
\end{aligned}
$$

for case II.

It may be noticed that the shape functions for the extension and flexure are the same as those for a standard beam while for torsion and shear lag they are the same as those of beam-columns. For distortional warping, the shape functions are analogous to the shape functions of beam-columns on elastic foundation. For this reason in the literature pertaining to box girders, sometimes reference is made to beam on elastic foundation analogy analysis of box girders (Wright and Samad 1970).

\subsection{Finite Element Formulation}

We shall now derive the stiffness matrix and nodal load vector of a multicell thin-walled box girder based on the foregoing displacement functions.

\subsubsection{Element Geometry and Coordinate System}

Fig. 3.3 shows the geometry and coordinate system of a typical box beam element with two end nodes $i$ and $j$. The generalized nodal displacement vector corresponding to $\{\bar{D}\}$ in Eq. (3.3.10) is given by

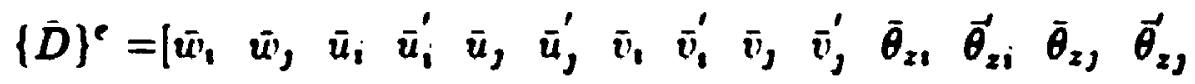

$$
\begin{aligned}
& \bar{\beta}_{11}, \bar{\beta}_{1}^{\prime}, \bar{\beta}_{1}, \bar{\beta}_{1}^{\prime}, \ldots, \bar{\beta}_{n i} \bar{\beta}_{n i}^{\prime} \bar{\beta}_{n,} \bar{\beta}_{n j}^{\prime} \\
& \left.\bar{\psi}_{1,} \bar{\psi}_{1 i}^{\prime} \bar{\psi}_{1 j}, \bar{\psi}_{1 j}^{\prime}, \ldots, \bar{\psi}_{m i} \bar{\psi}_{m i}^{\prime} \bar{\psi}_{m}, \bar{\psi}_{m,}^{\prime}\right]^{T}
\end{aligned}
$$

where $\{\bar{D}\}^{e}$ is the vector of element nodal degrees of freedom. Using standard finite element procedures, the element displacements can be related to its nodal displacements as

$$
\{\bar{D}\}=[N]\{\bar{D}\}^{e}
$$




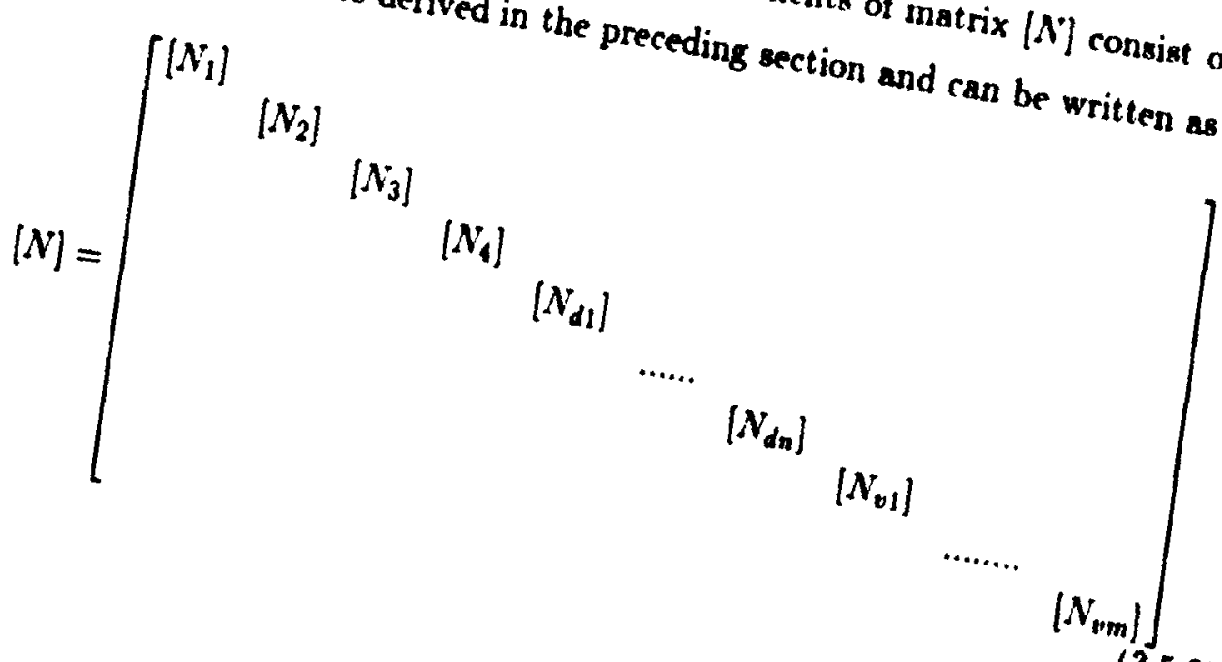
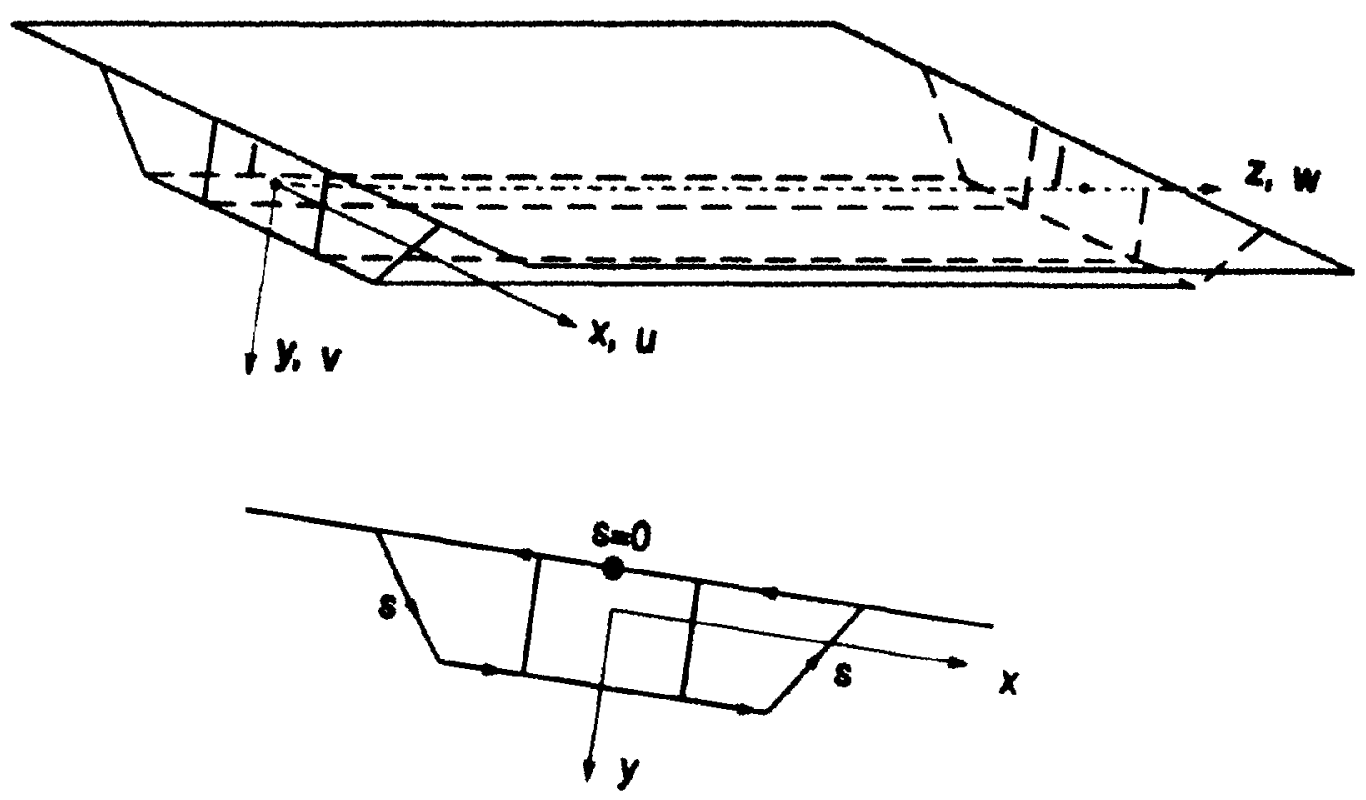

Fig. 3.3 Geometry of multicell box beam element.

$$
\begin{aligned}
& \text { 3.5.2 Stress-Scrain Relation } \\
& \text { The normal strain } \epsilon_{2} \text { and shear strain } \gamma_{a x} \text { at any point on the bearn can be } \\
& \text { written as } \\
& \qquad \epsilon_{2}=-\{\omega\}^{T}\left\{D^{\prime \prime}\right\}=-\{\omega\}^{T}\left\{D^{\prime \prime}\right)
\end{aligned}
$$




$$
\gamma_{s x}=\{\bar{\gamma}\}^{T}\left\{D^{\prime}\right\}=\{\bar{F}\}^{T}\left\{\bar{D}^{\prime}\right\}
$$

Substituting Eq. (3.5.2) in to Eqs. (3.5.4) and (3.5.5), it can be written that

$$
\{\epsilon\}=\left[\epsilon_{z} \gamma_{s z}\right]^{T}=[B]\{\ddot{D}\}^{e}
$$

where $[B]$ is the usual strain-displacement matrix

$$
[B]=\left\{\begin{array}{c}
-\{\bar{\omega}\}^{T}\left[N^{\prime \prime}\right] \\
\{\overline{\bar{\tau}}\}^{T}\left[N^{\prime}\right]
\end{array}\right\}
$$

Using Hooke's law, the normal stress $\sigma_{z}$ and shear stress $\tau_{g z}$ is given by

$$
\left\{\begin{array}{c}
\sigma_{2} \\
\tau_{s z}
\end{array}\right\}=\left[\begin{array}{ll}
E & 0 \\
0 & G
\end{array}\right]\left\{\begin{array}{c}
\epsilon_{z} \\
\gamma_{s z}
\end{array}\right\}
$$

or

$$
\{\sigma\}=[E]\{\epsilon\}=[E][B]\{\bar{D}\}^{e}
$$

\subsubsection{Stiffness Matrix and Equivalent Nodal Force Vector}

By the standard procedure of finite element method as outlined in section (3.2), the element stiffness matirx can be found as

$$
\begin{aligned}
{[\bar{S}]^{e} } & =\int_{V}[B]^{T}[E][B] d v+\int_{0}^{l}[N]^{T}[\bar{H}][N] d z \\
& =\int_{0}^{l}\left(\left[N^{\prime \prime}\right]^{T}[\bar{C}]\left[N^{\prime \prime}\right]+\left[N^{\prime}\right]^{T}[\bar{J}]\left[N^{\prime}\right]+[N]^{T}[\bar{H}][N]\right) d z
\end{aligned}
$$

Since $[\ddot{C}],[\bar{J}]$ and $[\bar{H}]$ are uncoupled diagonal matrices, the element stiffness matrix has the form

$$
[\bar{S}]^{e}=\left[\begin{array}{lllllll}
{\left[\bar{S}_{1}\right]} & & & & & & \\
& {\left[\bar{S}_{2}\right]} & & & & & \\
& & {\left[\bar{S}_{3}\right]} & & & & \\
& & & \ldots \ldots & & & \\
& & & & {\left[\bar{S}_{1}\right]} & & \\
& & & & & \ldots \ldots . & \\
& & & & & & {\left[\bar{S}_{n+m+1}\right]}
\end{array}\right]
$$

where

$$
\left[S_{1}\right]=\int_{0}^{l}\left(\left[N^{\prime \prime}\right]_{1}^{T} \bar{C}_{11}\left[N_{1}^{\prime \prime}\right]+\left[N_{1}^{\prime}\right]^{T} \bar{J}_{11}\left[N_{1}^{\prime}\right]+\left[N_{1}\right]^{T} \bar{H}_{i 1}\left[N_{i}\right]\right) d z
$$


is the stiffness matrix corresponding to the individual structural action modes.

Similarly, the equivalent element nodal force vector can be obtained as

$$
\begin{aligned}
\{\bar{F}\}^{e}= & \int_{0}^{l}\left(q_{x}[N]^{T}\left\{\bar{r}_{x}^{*}\right\}+q_{y}[N]^{T}\left\{\bar{r}_{y}^{*}\right\}-\int_{x} q_{x}\left[N^{\prime}\right]^{T}\{\bar{\omega}\} d s\right) d z+ \\
& \sum_{k}\left(P_{x}[N]^{T}\left\{\bar{r}_{x}^{*}\right\}+P_{y}[N]^{T}\left\{\bar{r}_{y}^{*}\right\}+P_{z}\left[N^{\prime}\right]^{T}\{\bar{\omega}\}\right)
\end{aligned}
$$

The equivalent loads will consist of the usual forces and moments and of a series of bimoments corresponding to torsional, distortional warping and shear lag displacements. For some common types of loading, the general procedure for determining the equivalent nodal load vector corresponding to each structural action mode is follows.

Distributed load $q_{1}(z)$ acting from $z_{1}$ to $z_{2}$, Fig. 3.4,

$$
\left\{\bar{F}_{1}\right\}=\int_{z_{1}}^{z_{2}}\left[N_{1}\right] q_{1}(z) d z
$$

Distributed moment $m_{i}(z)$ ecting from $z_{1}$ to $z_{2}$, Fig. 3.5,

$$
\left\{\bar{F}_{1}\right\}=\int_{2_{1}}^{z_{2}}\left[N_{1}^{\prime}\right] m_{1}(z) d z
$$

Concentrated load $P_{0}$ at $z=z_{0}$, Fig. 3.6,

$$
\left\{\bar{F}_{2}\right\}=P_{0}\left|N_{1}\right|_{2=x_{0}}^{T}
$$

Concentrated moment $M_{0}$ at $z=z_{0}$, Fig. 3.7,

$$
\left\{\bar{F}_{2}\right\}=M_{0}\left[N_{1}^{\prime}\right]_{2=2_{0}}^{T}
$$

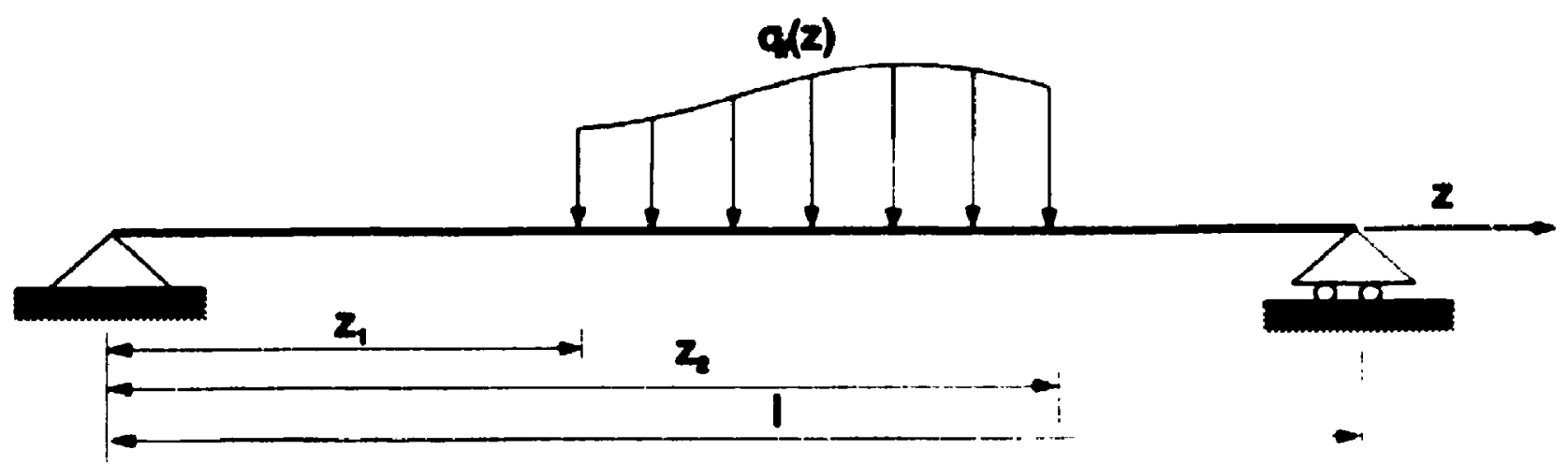

Fig. 3.4 Distributed load $q_{\imath}(z)$. 


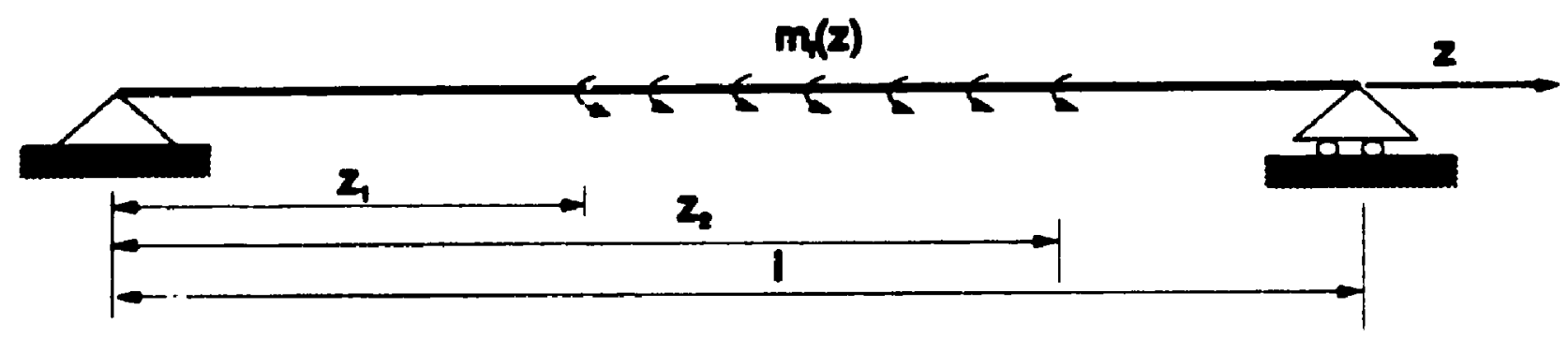

Fig. 3.5 Distributed moment $m_{i}(z)$.

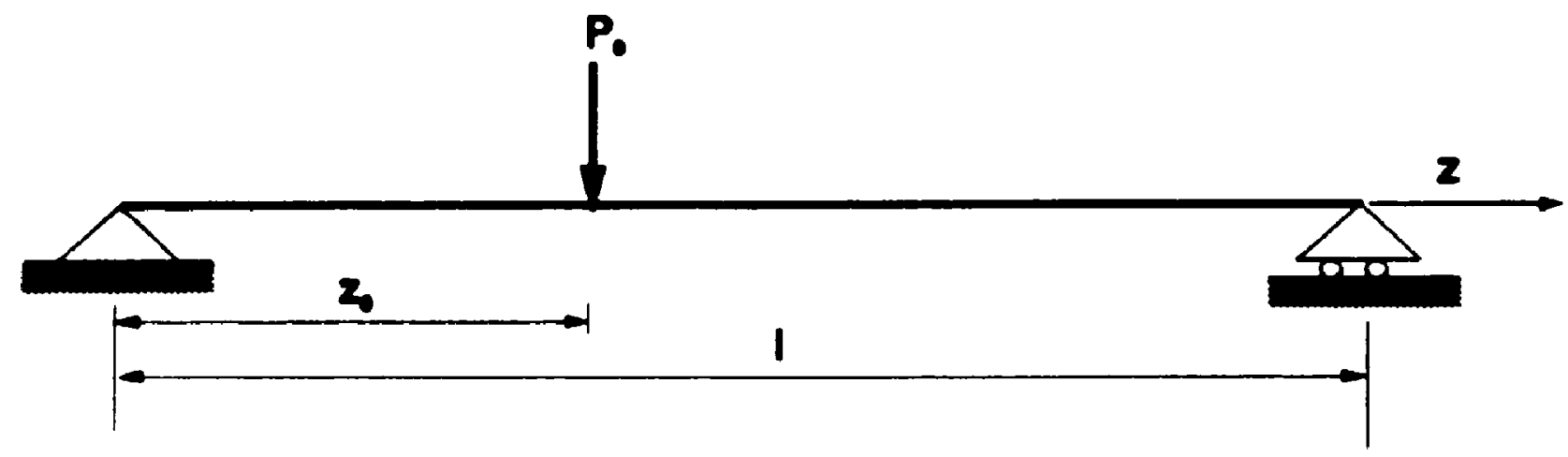

Fig. 3.6 Concentrated point load $P_{0}$.

By considering the uncoupled stiffnesses in Eq. (3.5.11), for each structural action mode, the following relationship can be obtained

$$
\left[\bar{S}_{i}\right]\left\{\bar{D}_{1}\right\}^{e}=\left\{\bar{F}_{i}\right\}
$$

The element stiffness matrix and equivalent nodal load vector for each structural action mode, as expressed by Eq. (3.5.18), can be explicitly determined as follows:

\section{Extension}

The element stiffness

$$
\left[\tilde{S}_{1}\right]=\left[\begin{array}{cc}
E A / l & -E A / l \\
-E A / l & E A / l
\end{array}\right]
$$




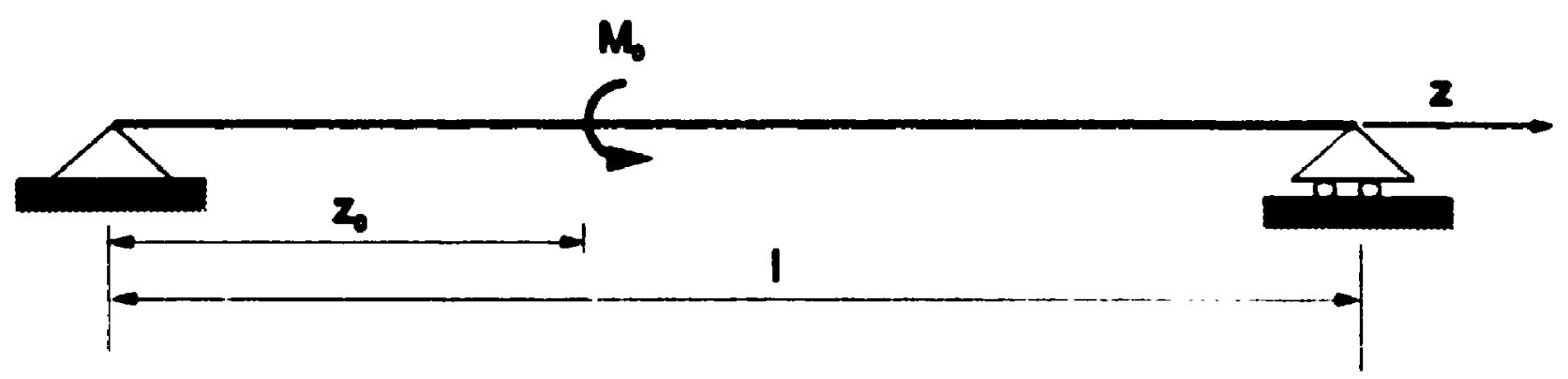

Fig. 3.7 Concentrated moment $M_{0}$.

The equivalent nodal load vector due to linearly distributed load

$$
q_{z}=m z+n
$$

where $m$ and $n$ are constants and are not related to the previous definitions of $m$ and $n$, from $z_{1}$ to $z_{2}$ is given by

$$
\left\{\bar{F}_{1}\right\}=\left\{\begin{array}{c}
\left(m z_{2}^{2} / 2-n z_{2}^{2} / l+n z_{2}^{2}-m z_{2}^{3} / 3 l\right)-\left(m z_{1}^{2} / 2-n z_{1}^{2} / l+n z_{1}^{2}-m z_{1}^{3} / 3 l\right) \\
\left(m z_{2}^{3} / 3 l+n z_{2}^{2} / 2 l\right)-\left(m z_{1}^{3} / 3 l+n z_{1}^{2} / 2 l\right)
\end{array}\right\}
$$

The nodal load vector due to axial concentrated load $P_{20}$ at $z=z_{0}$ is given by

$$
\left\{\bar{F}_{1}\right\}=P_{20}\left\{\begin{array}{c}
1-z_{0} / l \\
z_{0} / l
\end{array}\right\}
$$

\section{Flexure}

The element stiffness matrix

$$
\left[\bar{S}_{2}\right]=\frac{E I_{y}}{l}\left[\begin{array}{cccc}
12 / l^{2} & 6 / l & -12 / l^{2} & 6 / l \\
6 / l & 4 & -6 / l & 2 \\
-12 / l & -6 / l & 12 / l & -6 / l \\
6 / l & 2 & -6 / l & 4
\end{array}\right]
$$

For flexure in direction $x$, while the stiffness matrix $\left[\dot{S}_{3}\right]$, i.e. corresponding to bending about the $y$-axis, can be found by replacing $I_{y}$ with $I_{z}$ in the above equation. The nodal load vectors due to different loading conditions are given by: 
For linearly distributed load $q(z)=m z+n$ from $z_{1}$ to $z_{2}$ :

$$
\left\{\bar{F}_{2}\right\} \text { or }\left\{\bar{F}_{3}\right\}=\left\{\begin{array}{l}
a_{1} \\
a_{2} \\
a_{3} \\
a_{4}
\end{array}\right\}
$$

where

$$
\begin{gathered}
a_{1}=\left(m z_{2}^{2} / 2-3 m z_{2}^{4} / 4 l^{2}+2 m z_{2}^{3} / 5 l^{3}+n z_{2}-n z_{2}^{3} / l^{2}+n z_{2}^{4} / 4 l^{3}\right)- \\
\left(m z_{1}^{2} / 2-3 m z_{1}^{4} / 4 l^{2}+2 m z_{1}^{5} / 5 l^{3}+n z_{1}-n z_{1}^{3} / l^{2}+n z_{1}^{4} / 4 l^{3}\right) \\
a_{2}=\left(m z_{2}^{3} / 3-2 m z_{2}^{4} / 4 l+m z_{2}^{6} / 6 l^{3}+n z_{2}^{2} / 2-2 n z_{2}^{3} / 3 l+n z_{2}^{5} / 5 l^{3}\right)- \\
\left(m z_{1}^{3} / 3-2 m z_{1}^{4} / 4 l+n t z_{1}^{6} / 6 l^{3}+n z_{1}^{2} / 2-2 n z_{1}^{3} / 3 l+n z_{1}^{5} / 5 l^{3}\right) \\
a_{3}=\left(3 m z_{2}^{4} / 4 l^{2}-2 m z_{2}^{5} / 5 l^{3}+n z_{2}^{3} / l^{2}-n z_{2}^{4} / 2 l^{3}\right)- \\
\left(3 m z_{1}^{4} / 4 l^{2}-2 m z_{1}^{5} / 5 l^{3}+n z_{1}^{3} / l^{2}-n z_{1}^{4} / 2 l^{3}\right) \\
a_{4}=\left(m z_{2}^{5} / 5 l^{2}-m z_{2}^{4} / 4 l+n z_{2}^{4} / 4 l^{2}-n z_{2}^{3} / 3 l\right)- \\
\left(m z_{1}^{5} / 5 l^{2}-m z_{1}^{4} / 4 l+n z_{1}^{4} / 4 l^{2}-n z_{1}^{3} / 3 l\right)
\end{gathered}
$$

For uniformly distributed moment $m_{0}$ from $z_{1}$ to $z_{2}$ :

$$
\left\{\bar{F}_{2}\right\} \text { or }\left\{\bar{F}_{3}\right\}=m_{0}\left\{\begin{array}{c}
\left(-3 z_{2}^{2} / l^{2}+2 z_{2}^{3} / l^{3}\right)-\left(-3 z_{1}^{2} / l^{2}+2 z_{1}^{3} / l^{3}\right) \\
\left(z_{2}-2 z_{2}^{2} / l+z_{2}^{4} / l^{3}\right)-\left(z_{1}-2 z_{1}^{2} / l+z_{1}^{4} / l^{3}\right) \\
\left(3 z_{2}^{2} / l^{2}-2 z_{2}^{3} / l^{3}\right)-\left(3 z_{1}^{2} / l^{2}-2 z_{1}^{3} / l^{3}\right) \\
\left(z_{2}^{3} / l^{2}-z_{2}^{2} / l\right)-\left(z_{1}^{3} / l^{2}-z_{1}^{2} / l\right)
\end{array}\right\}
$$

For concentrated load $P_{0}$ at $z=z_{0}$ :

$$
\left\{\bar{F}_{2}\right\} \text { or }\left\{\bar{F}_{3}\right\}=P_{0}\left\{\begin{array}{c}
1-3 z_{0}^{2} / l^{2}+2 z_{0}^{3} / l^{3} \\
z_{0}\left(1-2 z_{0} / l+z_{0}^{3} / l^{3}\right) \\
3 z_{0}^{2} / l^{2}-2 z_{0}^{3} / l^{3} \\
z_{0}\left(z_{0}^{2} / l^{2}-z_{0} / l\right)
\end{array}\right\}
$$

For concentrated moment $M_{0}$ at $z=z_{0}$ :

$$
\left\{\bar{F}_{2}\right\} \text { or }\left\{\bar{F}_{3}\right\}=M_{0}\left\{\begin{array}{c}
-6 z_{0} / l^{2}+6 z_{0}^{2} / l^{3} \\
1-4 z_{0} / l+4 z_{0}^{3} / l^{3} \\
6 z_{0} / l^{2}-6 z_{0}^{2} / l^{3} \\
3 z_{0}^{2} / l^{2}-2 z_{0} / l
\end{array}\right\}
$$

Torsion and shear lag

The element stifness matrix

$$
\left[\bar{S}_{4}\right] \text { or }\left[\bar{S}_{v 1}\right]=\left[\begin{array}{llll}
\bar{S}_{11} & & & \\
\bar{S}_{21} & \bar{S}_{22} & & \\
\bar{S}_{31} & \bar{S}_{32} & \bar{S}_{33} & \\
\bar{S}_{41} & \bar{S}_{42} & \bar{S}_{43} & \bar{S}_{44}
\end{array}\right]
$$


where

$$
\begin{aligned}
& \bar{S}_{11}=\bar{S}_{33}=-\bar{S}_{31}=\frac{2\left(s_{4}+s_{3}\right)}{l^{2}}+\frac{\mathcal{N}}{l} \\
& \bar{S}_{21}=\bar{S}_{41}=-\bar{S}_{32}=-\bar{S}_{43}=\frac{s_{4}+s_{5}}{l} \\
& \bar{S}_{22}=\bar{S}_{44}=s_{4} \\
& \bar{S}_{42}=s_{5}
\end{aligned}
$$

in which $\quad s_{4}=\frac{\zeta_{1}\left(\zeta_{1} \cosh \zeta_{1}-\sinh \zeta_{1}\right)}{\left(2-2 \cosh \zeta_{1}+\zeta_{1} \sinh \zeta_{1}\right)} \frac{E I}{l}$

$$
s_{5}=\frac{\zeta_{1}\left(\sinh \zeta_{1}-\zeta_{1}\right)}{\left(2-2 \cosh \zeta_{1}+\zeta_{1} \sinh \zeta_{1}\right)} \frac{E I}{l}
$$

and $\zeta_{1}=l \zeta$. The nodal load vectors due to different loading conditions are given by For linearly distributed torsional moment or generalized shear lag load $q(z)=n z+n$ from $z_{1}$ to $z_{2}$ :

$$
\left\{\bar{F}_{4}\right\} \text { or }\left\{\bar{F}_{v_{1}}\right\}=|Q|^{T}\left\{\begin{array}{l}
a_{5} \\
a_{6} \\
a_{6} \\
a_{7}
\end{array}\right\}
$$

where

$$
\begin{gathered}
a_{5}=\left(m z_{2} e^{\zeta z_{2}} / \zeta-m e^{\zeta z_{2}} / \zeta^{2}+n e^{\zeta z_{2}} / \zeta\right)- \\
\left(m z_{1} e^{\zeta z_{1}} / \zeta-m e^{\zeta z_{1}} / \zeta^{2}+n e^{\zeta z_{1}} / \zeta\right) \\
a_{6}=\left(-m z_{2} e^{-\zeta z_{2}} / \zeta+m e^{-\zeta z_{2}} / \zeta^{2}-n e^{-\zeta z_{2}} / \zeta\right)- \\
\left(-m z_{1} e^{-\zeta z_{1}} / \zeta+m e^{-\zeta z_{1}} / \zeta^{2}-n e^{-\zeta z_{1}} / \zeta\right) \\
a_{7}=z_{2}^{2} / 2-z_{1}^{2} / 2 \\
a_{8}=z_{2}-z_{1}
\end{gathered}
$$

For uniformly distributed torsional bimoment or generalized shear lag moment $m_{0}$ from $z_{1}$ to $z_{2}$ :

$$
\left\{\bar{F}_{1}\right\} \text { or }\left\{\bar{F}_{v_{1}}\right\}=m_{0}[Q]^{T}\left\{\begin{array}{c}
\epsilon^{\zeta x_{2}}-\epsilon^{\zeta x_{1}} \\
e^{-\zeta x_{2}}-e^{-\zeta x_{1}} \\
z_{2}-z_{1} \\
0
\end{array}\right\}
$$

For concentrated torsional moment or generalized shear lag load $P_{0}$ at $z=z_{0}$ :

$$
\left\{\tilde{F}_{4}\right\} \text { or }\left\{\bar{F}_{u_{3}}\right\}=P_{0}[Q]^{T}\left\{\begin{array}{c}
e^{\zeta z_{0}} \\
e^{-\gamma_{0}} \\
z_{0} \\
1
\end{array}\right\}
$$


For concentrated torsional bimoment or shear lag moment $M_{0}$ at $z=z_{0}$ :

$$
\left\{\bar{F}_{1}\right\} \text { or }\left\{\bar{F}_{v 1}\right]=M_{0}[Q]^{T}\left\{\begin{array}{c}
\zeta e^{i x_{0}} \\
-\zeta e^{-\zeta x_{0}} \\
1 \\
0
\end{array}\right\}
$$

\section{Distortion}

The element stiffness matrix for distortional mode depends on the relative magnitude of the coefficients of $\mathrm{Eq}$. (3.4.14), but it can be written in the general form as follows

$$
\left[\bar{S}_{d i}\right]=\left[\begin{array}{llll}
\bar{S}_{11} & & & \\
\bar{S}_{21} & \bar{S}_{22} & & \\
\bar{S}_{31} & \bar{S}_{32} & \bar{S}_{33} & \\
\bar{S}_{41} & \bar{S}_{42} & \bar{S}_{43} & \bar{S}_{44}
\end{array}\right]
$$

where for case I

$$
\begin{aligned}
& \bar{S}_{11}=\bar{S}_{33}=2 E \bar{I}_{d i}\left[\lambda_{1}\left(\lambda_{1}^{2}-\gamma^{2}\right)\left(\frac{\gamma c_{1} s_{1}+\lambda_{1} c_{2} s_{2}}{\Delta}\right)\right] \\
& \bar{S}_{12}=-\bar{S}_{34}=-2 E \bar{I}_{d i}\left(\frac{\left(\lambda_{1}^{2}+\gamma^{2}\right)}{2}+\lambda_{1}^{2} \gamma\left(\frac{c_{1}^{2} s_{2}^{2}-s_{1}^{2} C_{2}^{2}}{\Delta}\right)\right] \\
& \bar{S}_{13}=2 E \bar{I}_{d i}\left[\lambda_{1}\left(\gamma^{2}-\lambda_{1}^{2}\right)\left(\frac{\gamma c_{2} s_{2}+\lambda_{1} s_{2} c_{1}}{\Delta}\right)\right] \\
& \bar{S}_{14}=-\bar{S}_{23}=-2 E \bar{I}_{d i}\left[\lambda_{1}\left(\gamma^{2}-\lambda_{1}^{2}\right)\left(\frac{s_{1} s_{2}}{\Delta}\right)\right] \\
& \bar{S}_{22}=\bar{S}_{44}=2 E \bar{I}_{d i}\left[\lambda_{1}\left(\frac{\gamma c_{1} s_{1}-\lambda_{1} c_{2} s_{2}}{\Delta}\right)\right] \\
& \bar{S}_{24}=2 E \bar{I}_{d i}\left[\lambda_{1}\left(\frac{\lambda_{1} c_{1} s_{2}-\gamma c_{2} s_{1}}{\Delta}\right)\right]
\end{aligned}
$$

and for case II

$$
\begin{aligned}
& \bar{S}_{11}=\bar{S}_{33}=2 E \bar{I}_{d i}\left[\lambda_{1}\left(\lambda_{1}^{2}-\gamma_{1}^{2}\right)\left(\frac{\gamma_{1} c_{1} s_{1}+\lambda_{1} c_{3} s_{3}}{\Delta_{1}}\right)\right] \\
& \bar{S}_{12}=-\bar{S}_{34}=-2 E \bar{I}_{d i}\left(\frac{\left(\lambda_{1}^{2}+\gamma_{1}^{2}\right)}{2}+\lambda_{1}^{2} \gamma_{1}\left(\frac{c_{1}^{2} s_{3}^{2}-s_{1}^{2} c_{3}^{2}}{\Delta_{1}}\right)\right] \\
& \bar{S}_{13}=2 E \bar{I}_{d i}\left[\lambda_{1}\left(\gamma_{1}^{2}-\lambda_{1}^{2}\right)\left(\frac{\gamma_{1} c_{3} s_{3}+\lambda_{1} s_{3} c_{1}}{\Delta_{1}}\right)\right] \\
& \bar{S}_{14}=-\bar{S}_{23}=-2 E \bar{I}_{d i}\left[\lambda_{1}\left(\gamma_{1}^{2}-\lambda_{1}^{2}\right)\left(\frac{s_{1} s_{3}}{\Delta_{1}}\right)\right] \\
& \bar{S}_{22}=\bar{S}_{14}=2 E \bar{I}_{d i}\left[\lambda_{1}\left(\frac{\gamma_{1} c_{1} s_{1}-\lambda_{1} c_{3} s_{3}}{\Delta_{1}}\right)\right] \\
& \bar{S}_{24}=2 E \bar{I}_{d i}\left[\lambda_{1}\left(\frac{\lambda_{1} c_{1} s_{3}-\gamma_{1} c_{3} s_{1}}{\Delta_{1}}\right)\right]
\end{aligned}
$$

The nodal load vector due to a trapezoidal generalized distortional moment

$$
q_{d i}=m z+n
$$


acting from $z_{1}$ to $z_{2}$ is given by

$$
\left\{\bar{F}_{d i}\right\}=[Q 1]^{T}\left\{\begin{array}{c}
W+X+a+b+c \\
-Y+Z+c-d \\
Y+Z+d+c \\
-W+X+a-b
\end{array}\right\}
$$

where

$$
\begin{gathered}
W=\frac{m}{2 \gamma_{2}^{2}}\left(\cosh \gamma_{2} z_{1}-\cosh \gamma_{2} z_{2}+\gamma_{2} z_{2} \sinh \gamma_{2} z_{2}-\gamma_{2} z_{1} \sinh \gamma_{2} z_{1}\right) \\
X=\frac{m}{2 \lambda_{2}^{2}}\left(\cosh \lambda_{2} z_{1}-\cosh \lambda_{2} z_{2}+\lambda_{2} z_{2} \sinh \lambda_{2} z_{2}-\lambda_{2} z_{1} \sinh \lambda_{2} z_{1}\right) \\
Z=\frac{m}{2 \lambda_{2}^{2}}\left(\sinh \lambda_{2} z_{1}-\sinh \lambda_{2} z_{2}+\lambda_{2} z_{2} \cosh \lambda_{2} z_{2}-\lambda_{2} z_{1} \cosh \lambda_{2} z_{1}\right) \\
Y=\frac{m}{2 \gamma_{2}^{2}}\left(\sinh \gamma_{2} z_{1}-\sinh \gamma_{2} z_{2}+\gamma_{2} z_{2} \cosh \gamma_{2} z_{2}-\gamma_{2} z_{1} \cosh \gamma_{2} z_{1}\right) \\
a=\frac{n}{2 \lambda_{2}}\left(\sinh \lambda_{2} z_{2}-\sinh \lambda_{2} z_{1}\right) \\
b=\frac{n}{2 \gamma_{2}}\left(\sinh \gamma_{2} z_{2}-\sinh \gamma_{2} z_{1}\right) \\
c=\frac{n}{2 \lambda_{2}}\left(\cosh \lambda_{2} z_{2}-\cosh \lambda_{2} z_{1}\right) \\
d=\frac{n}{2 \gamma_{2}}\left(\cosh \gamma_{2} z_{2}-\cosh \gamma_{2} z_{1}\right)
\end{gathered}
$$

in which $\quad \lambda_{2}=\lambda_{1}+\gamma$

$$
\gamma_{2}=\lambda_{1}-\gamma
$$

The nodal load vector due to uniformly distributed generalized distortional bimoment $m_{0}$ from $z_{1}$ to $z_{2}$ is given by

$$
\left\{\bar{F}_{d i}\right\}=m_{0}[Q 1]^{T}\left\{\begin{array}{l}
\cosh \lambda_{1} z_{2} \cosh \gamma z_{2}-\cosh \lambda_{1} z_{1} \cosh \gamma z_{1} \\
\cosh \lambda_{1} z_{2} \sinh \gamma z_{2}-\cosh \lambda_{1} z_{1} \sinh \gamma z_{1} \\
\sinh \lambda_{1} z_{2} \cosh \gamma z_{2}-\sinh \lambda_{1} z_{1} \cosh \gamma z_{1} \\
\sinh \lambda_{1} z_{2} \sinh \gamma z_{2}-\sinh \lambda_{1} z_{1} \sinh \gamma z_{1}
\end{array}\right\}
$$

The same procedures can be used to the nodal load vector for case. II. The nodal load vectors due to concentrated load $P_{0}$ and concentrated moment $M_{0}$ can be found by Eqs. (3.5.16) and (3.5.17).

In order to obtain the displacement, shear force and bending moment at any point along a beam, we must use the complete displacement function of the beam, 
consisting of the displacements caused by the nodal displacements, $\{d\}$, plus the displacements caused by the applied loads acting on the same beam with its ends clamped. The latter displacements are referred to as the particular solution of the governing equations. We follow Razaqpur $(1986,1990)$ in writing the complete solution of these equations. The complete displacement functions for different structural action modes correponding to the governing differential equations (3.4.1a) to (3.4.1f) are discussed below.

\section{Extension}

The complete displacement function can be written as

$$
w=w_{1}+w_{2}
$$

where $w_{1}$ is the displacement caused by the nodal displacement, $\{d\}$ (j.e. the homogeneous solution); and $w_{2}$ is the displacement caused by the applied load on the the same beam with its ends clamped (i.e. the particular integral). According to Eq. (3.4.3)

$$
w_{1}=\left[N_{1}\right]\{d\}
$$

and

$$
w_{2}=A_{1}+A_{2} z+w_{p}
$$

with $w_{p}$ being a particular integral corresponding to the loading function $q_{z}(z)$, and for linearly distributed load in Eq. (3.5.20)

$$
w_{p}=\frac{1}{E A}\left(\frac{m z^{3}}{6}+\frac{n z^{2}}{2}\right)
$$

Therefore

$$
u_{2}=A_{1}+A_{2} z+\frac{1}{E A}\left(\frac{m z^{3}}{6}+\frac{n z^{2}}{2}\right)
$$

The constants $A_{1}$ and $A_{2}$ can be found using the boundary conditions of a beam clamped at both ends as follows

$$
\begin{aligned}
& A_{1}=0 \\
& A_{2}=-\frac{1}{E A}\left(\frac{m l^{2}}{6}+\frac{n l}{2}\right)
\end{aligned}
$$


Thus,

$$
w=\left\{N_{1}\right]\{d\}-\frac{z}{E A}\left(\frac{m l^{2}}{6}+\frac{n l}{2}\right)+\frac{1}{E A}\left(\frac{m z^{3}}{6}+\frac{n z^{2}}{2}\right)
$$

For concentrated load $P_{z 0}$ at $z=z_{0}:$ when $z \leq z_{0}$

$$
w_{2}=\frac{z_{0} P_{z 0}\left(l-z_{0}\right)}{l}\left(1-\frac{z}{l}\right)
$$

when $z>z c$

$$
w_{2}=\frac{z_{0} P_{20}\left(l-z_{0}\right)}{l}\left(\frac{z}{l}\right)
$$

The axial force at any point within the beam is given by

$$
N_{z}=E A w^{\prime \prime}
$$

\section{Flexure}

The complete deflection can be expressed as

$$
u=u_{1}+u_{2}
$$

where

$$
u_{1}=\left[N_{2}\right]\{d\}
$$

and

$$
u_{2}=A_{1} z^{3}+A_{2} z^{2}+A_{3} z+A_{4}+u_{p}
$$

in which, for linearly distributed load, $u_{p}$ is given by

$$
u_{p}=\frac{1}{E I_{y}}\left(\frac{m z^{5}}{120}+\frac{n z^{4}}{24}\right)
$$

Therefore

$$
u_{2}=[L] A+\frac{1}{E I_{y}}\left(\frac{m z^{5}}{120}+\frac{n z^{4}}{24}\right)
$$

where

$$
\begin{aligned}
& {[L]=\left[\begin{array}{llll}
z^{3} & z^{2} & z & 1
\end{array}\right]} \\
& \{A\}=\left[\begin{array}{llll}
A_{1} & A_{2} & A_{3} & A_{4}
\end{array}\right]^{T}
\end{aligned}
$$

Substitution of the appropriate boundary conditions into Eq. (3.5.54) leads to

$$
\{A\}=\{Q 0]\{J 0\}
$$


where

$$
\begin{gathered}
{[Q 0]=\left[\begin{array}{cccc}
0 & 0 & 0 & 1 \\
0 & 1 & 0 & 0 \\
l^{3} & l^{2} & l & 1 \\
3 l^{2} & 2 l & 1 & 0
\end{array}\right]^{-1}} \\
\{J 0\}=-\frac{1}{E I_{y}}\left[\begin{array}{llll}
0 & 0 & \left(m l^{5} / 120+n l^{4} / 24\right) & \left(m l^{4} / 24+n l^{3} / 6\right)
\end{array}\right]^{T}
\end{gathered}
$$

Hence, the complete deflection function can be written $\mathrm{s}$

$$
u=\left[N_{2}\right]\{d\}+[L][Q 0]\{J 0\}+\frac{1}{E I_{v}}\left(\frac{m z^{5}}{120}+\frac{n z^{4}}{24}\right)
$$

The shear force and bending moment are given by successive differentiation of this equation

$$
\begin{aligned}
& V(z)=-E I_{y} u^{\prime \prime \prime} \\
& M(z)=-E I_{y} u^{\prime \prime}
\end{aligned}
$$

For concentrated load $P_{0}$ at $z=z_{0}$, the deflection function, $u_{2}$, will have two equations, depending on whether $z \leq z_{0}$ or $z>z_{0}$. To obtain these equations, we treat the beam as an assemblage of two members, I and II, of length $z_{0}$ and $\left(l-z_{0}\right)$ with two degrees of freedom $d_{1}$ and $d_{2}$, as shown in Fig. 3.8. The stiffness equation corresponding to these two dergees of freeciom can be established as

$$
\left[\begin{array}{ll}
k_{11} & k_{12} \\
k_{21} & k_{22}
\end{array}\right]\left\{\begin{array}{l}
d_{1} \\
d_{2}
\end{array}\right\}=\left\{\begin{array}{c}
P_{0} \\
0
\end{array}\right\}
$$

or

$$
\left\{\begin{array}{l}
d_{1} \\
d_{2}
\end{array}\right\}=\frac{P_{0}}{k_{11} k_{22}-k_{12} k_{21}}\left\{\begin{array}{c}
k_{22} \\
-k_{21}
\end{array}\right\}
$$

where

$$
\begin{aligned}
& k_{11}=S_{33}^{l}+S_{11}^{I I} \\
& k_{12}=S_{34}^{I}+S_{12}^{I I} \\
& k_{21}=S_{43}^{I}+S_{21}^{I I} \\
& k_{22}=S_{44}^{I}+S_{22}^{I I}
\end{aligned}
$$

In the above the superscripts I and II denote members I and II, and the $S_{y}$ are the stiffness coefficients of each beam element. These can be calculnted using the 


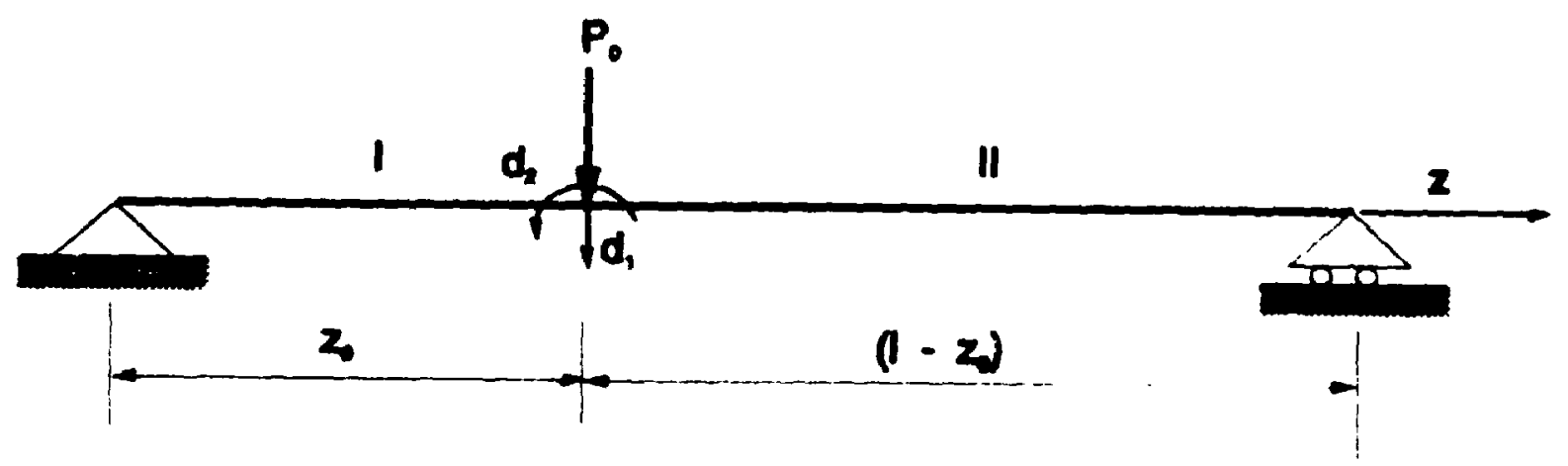

Fig. 3.8 Beam subjected to point load $P_{0}$ and idealized as two elements. expressions in Eq. (3.5.22), with the provision that I be replaced by zo for member I and by $\left(l-z_{0}\right)$ for member II.

Knowing $d_{1}$ and $d_{2}$ from Eq. (3.5.61), for $z \leq z_{0}$

$$
u_{2}=d_{1} N_{3}+d_{2} N_{1}
$$

while for $z>z_{0}$

$$
u_{2}=d_{1} N_{1}+d_{2} N_{2}
$$

When evaluating $N_{1}$ and $N_{2}$, we must substitute for $z,\left(z-z_{0}\right)$, or we could use $z$, provided $z$ is measured from the point of application of the point load.

For concentrated moment $M_{0}$ at $z=z_{0}$, the exart same procedures can be applied; accordingly

$$
\left\{\begin{array}{l}
d_{1} \\
d_{2}
\end{array}\right\}=\frac{M_{0}}{k_{11} k_{22}-k_{12} k_{21}}\left\{\begin{array}{c}
-k_{21} \\
k_{11}
\end{array}\right\}
$$

Torsion and shear lag

The complete solution of Eqs. (3.4.1d) and (3.4.1e) is given by

$$
y=y_{1}+y_{2}
$$

where according to Eq. (3.4.10)

$$
\left.y_{1}=\left[N_{1}\right]\{d\}=\mid L\right][Q]\{d\}
$$


and

$$
y_{2}=A_{1} e^{\zeta z}+A_{2} e^{-6 x}+A_{3} z+A_{4}+y_{p}=[L]\{A\}+y_{p}
$$

in which for the common case of a linearly varying load, $y_{p}$ is given by

$$
y_{p}=-\frac{1}{\mathcal{N}}\left(\frac{m z^{3}}{6}+\frac{n z^{2}}{2}\right)
$$

Thus,

$$
y_{2}=[L]\{A\}-\frac{1}{\mathcal{N}}\left(\frac{m z^{3}}{6}+\frac{n z^{2}}{2}\right)
$$

Substituting booundary conditions, the constant $\{A\}$ can be found

$$
\{A\}=[Q]\{J 1\}
$$

where

$$
\{J 1\}=\frac{1}{\mathcal{N}}\left[\begin{array}{llll}
0 & 0 & \left(m l^{3} / 6+n l^{2} / 2\right) & \left(m z^{2} / 2+n l\right)
\end{array}\right]^{T}
$$

Therefore

$$
y_{2}=[L][Q]\left\{J 1 j-\frac{1}{\mathcal{N}}\left(\frac{m z^{3}}{6}+\frac{n z^{2}}{2}\right)\right.
$$

The complete displacement function can be expressed

$$
y=\left[N_{i}\right]\{d\}+[L][Q]\{J 1\}-\frac{1}{\Lambda^{\prime}}\left(\frac{m z^{3}}{6}+\frac{n z^{2}}{2}\right)
$$

The shear force and bimoment are given by

$$
\begin{gathered}
V(z)=-E I_{y}{ }^{\prime \prime \prime}+\mathcal{N} y^{\prime} \\
M(z)=-E I_{y}{ }^{\prime \prime}
\end{gathered}
$$

The displacement function $y_{2}$ due to a concentrated load and a moment can be found by the same procedi. as discussed in the flexural case.

\section{Distortion}

The same procedures can be used for distortional Eq. (3.4.1f). Accordingly

$$
\overline{\beta_{1}}=\left[N_{d_{1}}\right]\{d\}
$$

and

$$
\overline{\beta_{2}}=\left[L \mid\{A\}+\overline{\beta_{p}}\right.
$$


where $[L]$ can be found from Eq. (3.4.21) for case I, and from Eq. (3.4.27) for case II. For linearly distributed generalized distortional moment in Eq. (3.5.39), $\bar{\beta}_{\text {p }}$ is given by

$$
\overline{\beta_{p}}=\frac{1}{\bar{H}_{1}}(m z+n)
$$

From the boundary conditions, the constants $\{A\}$ can be found

$$
\{A\}=[Q 1]\{J 2\}
$$

for case I, and for case II

$$
\{A\}=[Q 2]\{J 2\}
$$

where

$$
\{J 2\}=-\frac{1}{\bar{H}_{\imath}}\left[\begin{array}{llll}
n & m & (m l+n) & m
\end{array}\right]^{T}
$$

Hence, the complete distortional function for distortional mode $i$ can be expressed as follows

$$
\bar{\beta}_{1}=\bar{\beta}_{1}+\bar{\beta}_{2}
$$

For concentrated load and moment, $\overline{\beta_{2}}$ can be found in the same way as that in flexural case.

The shear force and bimoment are given by

$$
\begin{gathered}
V(z)=-E \bar{I}_{d z} \bar{\beta}_{\mathrm{z}}^{\prime \prime \prime}+G \bar{J}_{d_{z}} \bar{\beta}_{1}^{\prime} \\
M(z)=-E \bar{I}_{d_{1}} \bar{\beta}_{1}^{\prime \prime}
\end{gathered}
$$

Once the internal forces such as shear force and bending moment or bimoment have been found, the stresses can be calculated by the equations given in Chapter 2 . 


\section{VARIABLE DEPTH CURVED ELEMENT}

\subsection{General}

The element developed in Chapter 3 is highly efficient for straight box girder bridges, but it is not applicable to haunched or skew bridges. It can be applied to curved bridges by dividing the bridge into a number of straight segments. However, it is preferable to model a curved bridge by curved beam elements. Therefore, in this chapter a variable depth curved thin-walled box beam element will be devel-

oped based on the generalized curved thin-walled beam theory. The basic theory of curved thin-walled box beams based on the coupled flexural-torsional behavior will be introduced. The fundamental equations for flexure, pure torsion and torsional warping will be rigorously derived by taking into consideration the initial curvature of the curved beam. The governing differential equations of shear lag for a curved beam will be derived by means of Reissner's hypothesis and the principle of virtual work. Furthermore, the fundamental equations for distortional analysis will be also developed.

In Section (4.3) a generalized curved thin-walled multicell box beam theory will be presented based on an improved form of Vlasov's theory. For flexural analysis, Timoshenko's beam theory will be used in order to include shear deformations. The governing differential equations of extension, flexure, torsion, distortion and shear lag will be derived according to the theory of elasticity. Based on this theory, a variable depth curved multicell box beam element will be developed in Section (4.4). This curved element may be combined with the straight element developed in Chapter 3 in order to analyze box girder assemblages. 


\subsection{Basic Theory of Curved Thin-Walled Beams}

The bending and torsion of the curved thin-walled box beans can, in principle. be analyzed analogously to those of the straight box beams. However, because of the presence of initial curvature, bending and torsion are always coupled which makes the analysis more complicated. For simplicity, we assume that the cross section and the radius $R_{0}$ of a curved box beam are constant, but the theory can be easily extended to develop variable depth non-constant curvature element. Another assumption in the proposed development is that the ratio of initial curvature radius $R_{0}$ to the section width $B$ is great enough so that the box beam can be represented by its central axis.

\subsubsection{Flexure and Torsion}

The curvilinear coordinates $x, y$ and $z$ will be used, and $x$ and $y$ are the centroidal principal axes of the cross section as shown in Fig. 4.1.

\section{Equilibrium of forces.}

A curved beam subjected to a vertical distributed load $q_{y}$ and a distributed torsional moment $m_{\ell}$ is shown in Fig. 4.2. The equilibrium of forces and moments can be written as

$$
\begin{gathered}
Q_{y}+d Q_{y}-Q_{y}+q_{y} d z=0 \\
M_{x}+d M_{x}-M_{x}-Q_{y} R_{0} d \phi+T_{z} d \phi+\frac{q_{y}\left(R_{0} d \phi\right)^{2}}{2}=0 \\
T_{z}+d T_{z}-T_{z}-M_{x} d \phi+m_{t} d z=0
\end{gathered}
$$

or

$$
\begin{gathered}
\frac{d Q_{y}}{d z}=-q_{y} \\
\frac{d M_{x}}{d z}+\frac{T_{z}}{R_{0}}=Q_{y} \\
\frac{d T_{z}}{d z}-\frac{M_{x}}{R_{0}}=-m_{\ell}
\end{gathered}
$$




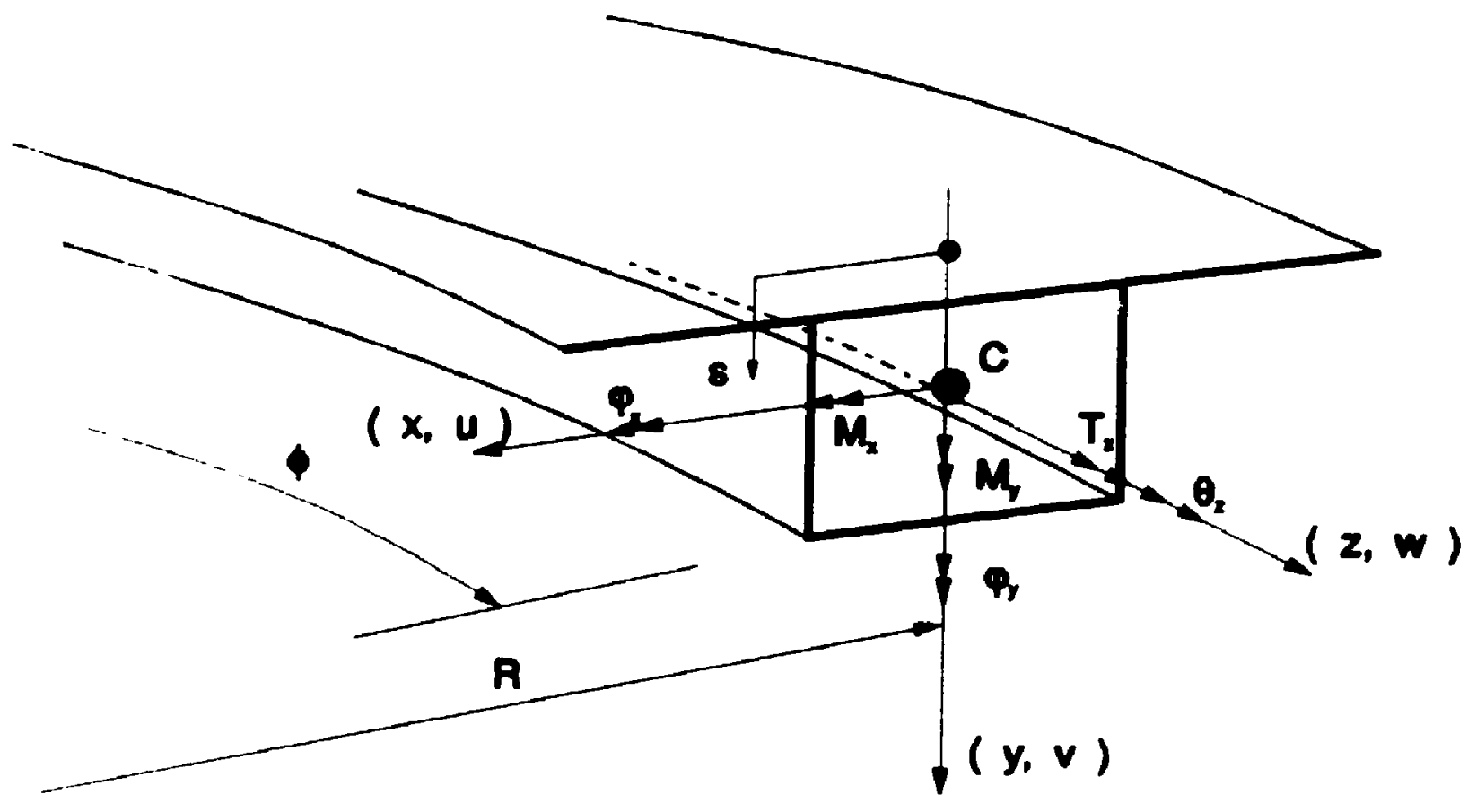

Fig. 4.1 Coordinate system for curve box beam.

where $Q_{y}, M_{x}$ and $T_{z}$ are the shear force, bending moment and torsional moment in $y, x$ and $z$ axes, respectively; while $\phi$ is the angular coordinate axis.

Differentiating Eq. (4.2.3a) with respect to the curvilinear coordinate $z$ and substituting Eq. (4.2.3b) into this equation, the following equation, which repesents the relationships between the bending moment $M_{x}$, load $q_{y}$, and torsional load $m_{t}$, can be obtained

$$
\frac{d^{2} M_{x}}{d z^{2}}+\frac{M_{x}}{R_{0}^{2}}=-q_{y}+\frac{m_{t}}{R_{0}}
$$

By comparison with the well-known equation

$$
\frac{d^{2} M_{z}}{d z^{2}}=-q_{y}
$$

for straight beams, the additional terms $M_{x} / R_{0}^{2}$ and $m_{t} / R_{0}$ in Eq. (4.2.4) are induced by the curvature of the beam.

Displacements and deformations.

The displacements $u(x, y, z)$ and $v(x, y, z)$ in the directions $x$ and $y$, respectively, 


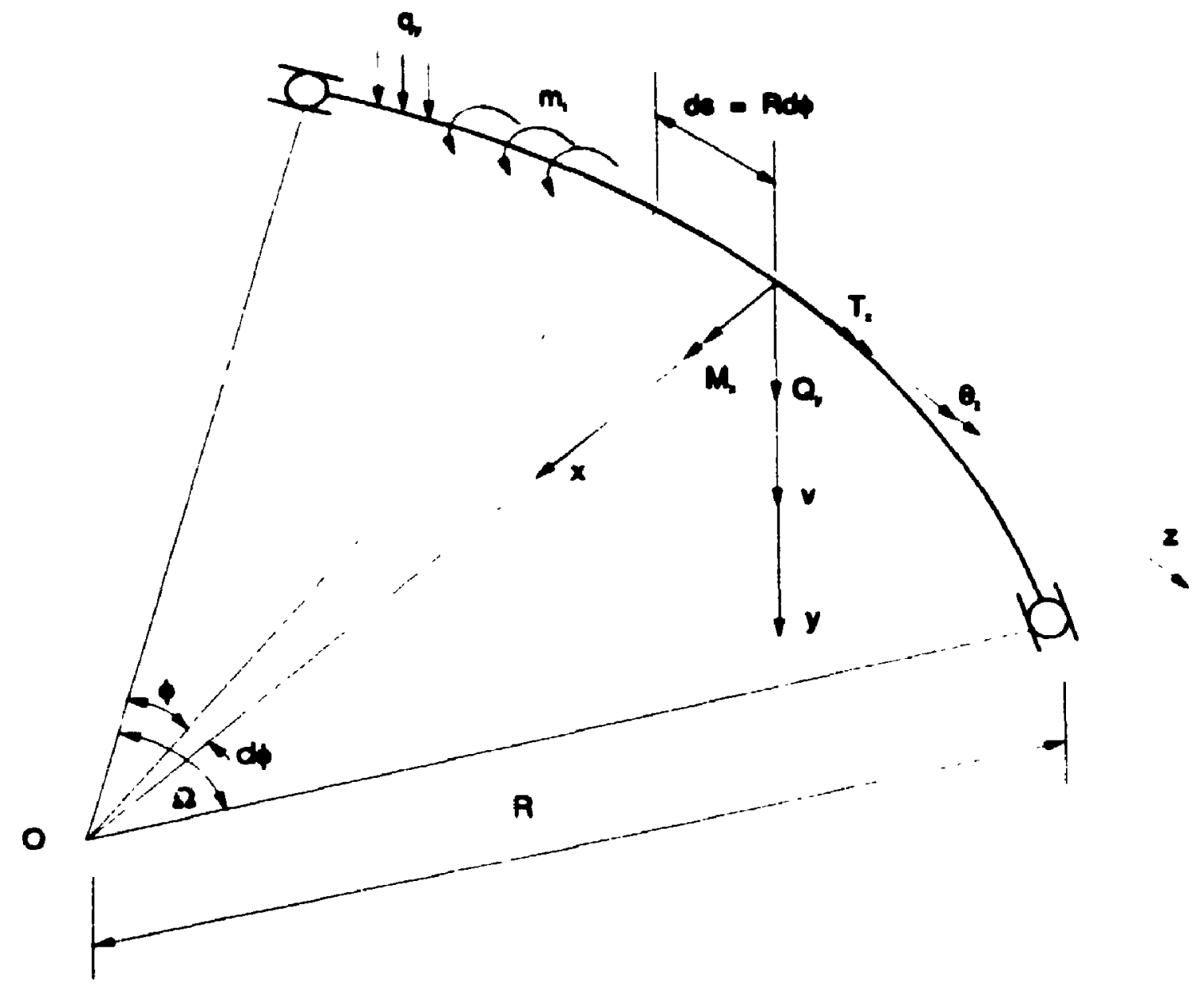

Fig. 4.2 Loads, forces, and displacements of a curve beam.

at any point $(x, y)$ of the section $z$ can be expressed in the following form:

$$
\begin{aligned}
& u(x, y, z)=u_{0}(z)-\left(y-y_{s}\right) \theta_{z} \\
& v(x, y, z)=v_{0}(z)-\left(x-x_{s}\right) \theta_{z}
\end{aligned}
$$

where $u_{0}(z)$ and $v_{0}(z)$ are the displacements of the centorid of the cross section in the direction of $x$ and $y$, respectively; $\theta_{z}$ is the angle of twist about the shear center $S$ of the cross section, and $x_{s}$ and $y_{s}$ are the shear center coordinates.

The total rotation due to bending about the $x$ axis, $\bar{\theta}_{x}$, is

$$
\bar{\theta}_{x}=-\frac{d v_{0}}{d z}=-v_{1}^{\prime}
$$

while the total rotation in the direction $y$ consists of $u_{0}^{\prime}$, due to the horizontal deflection, and $w_{0} / R_{0}$, due to the uniform axial displacement $w_{0}$, as shown in Fig. 4.3. Thus

$$
\bar{\theta}_{y}=u_{0}^{\prime}+x w_{0}
$$


where

$$
\chi=\frac{1}{R_{0}}
$$

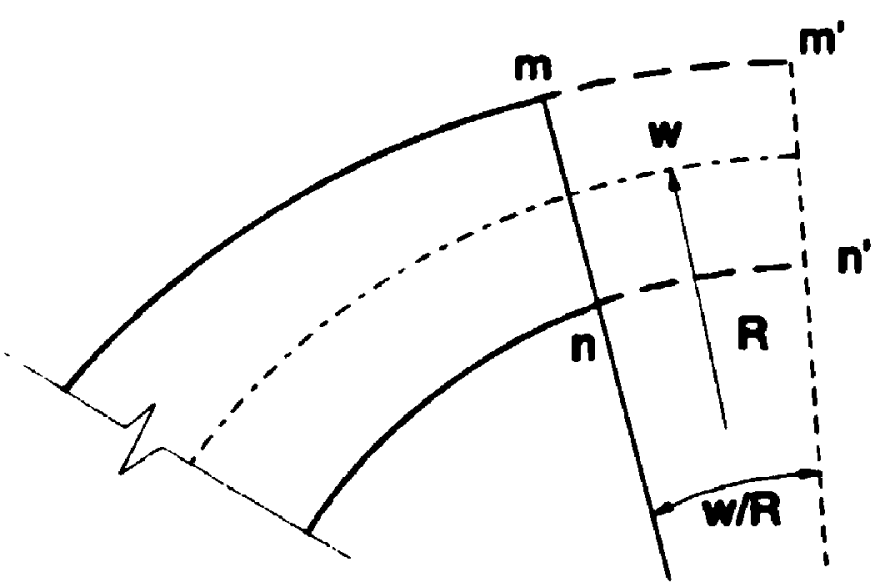

Fig. 4.3 Rotation due to uniform axial displacement $w$.

For the torsional angle of a curved beam, in addition to the rotation $\theta_{z}$ due to torsion, there is a contribution due to the vertical deflection $v_{0}$, as shown in Fig. 4.4. The total torsional angle $\bar{\theta}_{z}$ is therefore

$$
\bar{\theta}_{2}=\theta_{2}+\chi v_{0}
$$

Finally, similarly to the straight beam, the longitudinal displacement at any point $(x, y)$ on a cross section can be expressed as

$$
w(x, y, z)=w_{0}-x \bar{\theta}_{y}+y \bar{\theta}_{x}-\omega \vec{\theta}_{*}
$$

Equations (4.2.6), (4.2.7) and (4.2.12) enable us to determine the displacements of any point on the beam.

Strain, stress and stress resultants.

The normal strain in a curved box beam is given by

$$
\epsilon_{z}=\frac{\tilde{c} w(x, y, z)}{\partial z}-\frac{u(x, y, z)}{R_{0}}
$$

By substituting for $u(x, y, z)$ and $w(x, y, z)$ from Eqs. (4.2.6) and (4.2.12), respectively, we get

$$
\epsilon_{z}=u_{0}^{\prime}-x\left(u_{0}^{\prime \prime}+\lambda u_{0}^{\prime \prime}\right)-y\left(v_{0}^{\prime \prime}-\lambda \theta_{2}\right)-\omega\left(\theta_{8}^{\prime \prime}+\lambda v_{0}^{\prime \prime}\right)-\lambda\left(u_{0}+y_{s} \theta_{2}\right)
$$




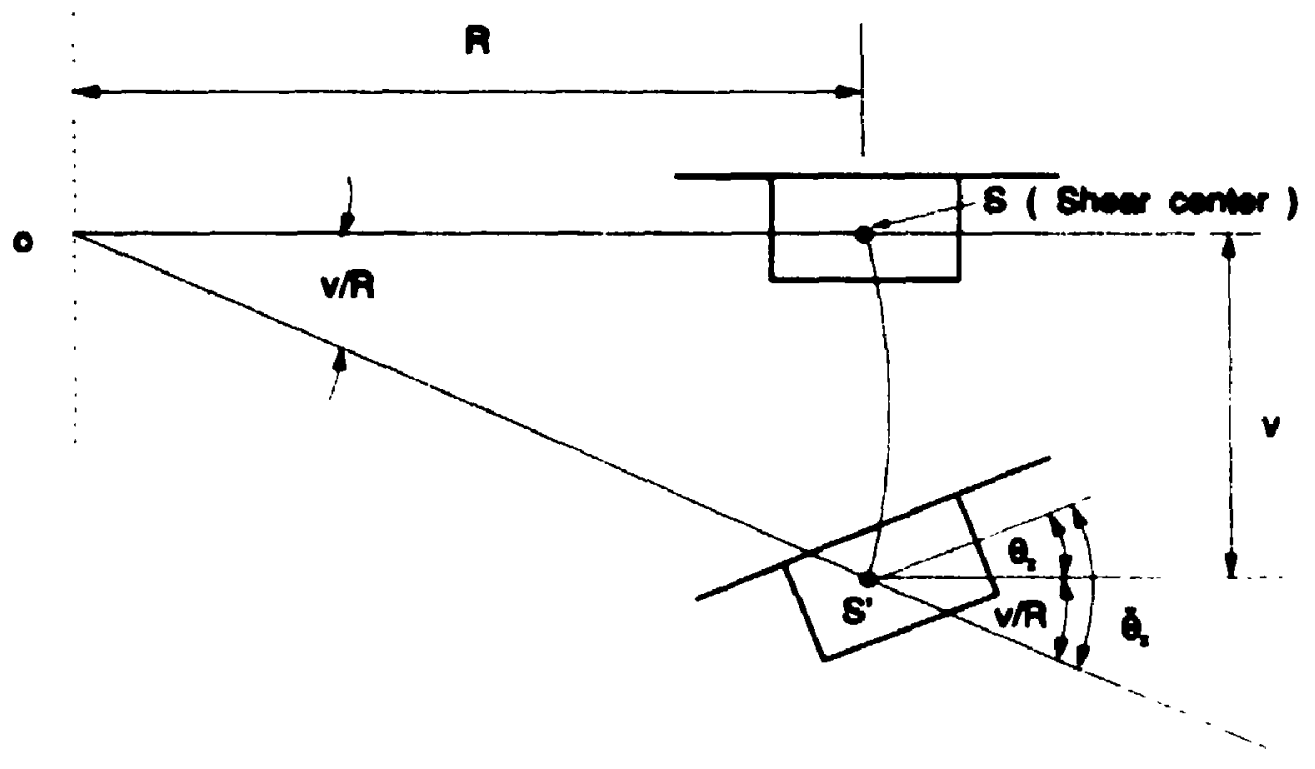

Fig. 4.4 Definition of torsional angle.

The corresponding normal stress can readily be found from Hooke's law

$$
\sigma_{z}=E \epsilon_{z}=E\left[w_{0}^{\prime}-x\left(u_{0}^{\prime \prime}+\chi w_{0}^{\prime \prime}\right)-y\left(v_{0}^{\prime \prime}-\chi \theta_{z}\right)-\omega\left(\theta_{z}^{\prime \prime}+\chi v_{0}^{\prime \prime}\right)-\lambda\left(u_{0}+y_{s} \theta_{z}\right)\right]
$$

Since $x, y, z$ and $\omega$ are orthogonalized principal coordinates, the stress resultants corresponding to those coordinates can be obtained from the following equilibriun conditions:

$$
\begin{aligned}
& N_{z}=\int_{A} \sigma_{z} d A=E \cdot 1\left[u_{0}^{\prime}-\chi\left(u_{0}+y_{s} \theta_{z}\right)\right] \\
& M_{x}=\int_{A} \sigma_{z} y d A=-E I_{x}\left(v_{0}^{\prime \prime}-\chi \theta_{z}\right) \\
& M_{y}=\int_{A} \sigma_{z} x d A=-E I_{y}\left(u_{0}^{\prime \prime}+\chi w_{0}^{\prime \prime}\right) \\
& M_{\omega}=\int_{A} \sigma_{z} \omega d A=-E I_{\omega}\left(\theta_{z}^{\prime \prime}+\chi v_{0}^{\prime \prime}\right)
\end{aligned}
$$

where $M_{\omega}$ is as usual the bimoment.

Substituting Eqs. (4.2.15a) to (4.2.15d) in to Eq. (4.2.14' the latter reduces to

$$
\sigma_{z}=\frac{N_{z}}{A}+\frac{M_{y}}{I_{y}} x+\frac{M_{x}}{I_{x}} y+\frac{M_{\omega}}{I_{\omega}} \omega
$$

which has the same form as that of straight box beam.

The remaining stress resultants, i.e., the pure torsional moment $T_{0}$ and the secondary torsional mornent $T_{\omega}$ can be obtained from 


$$
\begin{aligned}
& T_{s}=G J \frac{d \overline{\theta_{z}}}{d z}=G J\left(\frac{d \theta_{z}}{d z}+\frac{1}{R_{0}} \frac{d v_{0}}{d z}\right) \\
& T_{\omega}=-E I_{\omega} \frac{d^{3} \bar{\theta}_{z}}{d z^{3}}=-E I_{\omega}\left(\frac{d^{3} \theta_{z}}{d z^{3}}+\frac{1}{R_{0}} \frac{d^{3} v_{0}}{d z^{3}}\right)
\end{aligned}
$$

The total torsional moment, $T_{\varepsilon}$, is

$$
T_{2}=T_{s}+T_{w}
$$

In the special case when $w_{0}=u_{0}=0$, which is common for box girder bridges, the following governing differential equations can be derived by substituting Eq. (4.2.15b), and Eqs. (4.2.17) to (4.2.19) into Eqs. (4.2.3a) and (4.2.3b):

$$
\begin{gathered}
\left(E I_{x}+\frac{E I_{\omega}}{R_{0}^{2}}\right) \frac{d^{4} v_{0}}{d z^{4}}+\frac{E I_{\omega}}{R_{0}} \frac{d^{4} \theta_{z}}{d z^{4}}-\frac{G J_{3}}{R_{0}} \frac{d^{2} v_{0}}{d z^{2}}-\frac{E I_{x}+G J_{3}}{R_{0}} \frac{d^{2} \theta_{z}}{d z^{2}}=q_{y}+\frac{m_{t}}{R_{0}} \\
E I_{\omega} \frac{d^{4} \theta_{z}}{d z^{4}}+\frac{E I_{\omega}}{R_{0}} \frac{d^{4} v_{0}}{d z^{4}}-G J_{s} \frac{d^{2} \theta_{z}}{d z^{2}}+\frac{E I_{x}-G J_{3}}{R_{0}} \frac{d^{2} v_{0}}{d z^{2}}+\frac{E I_{x} \theta_{z}}{R_{0}^{2}}=m_{t}
\end{gathered}
$$

The shear stress in the curved box beam caused by pure torsion, warping torsion and bending can be determir 1 similarly to the straight box beam (see Chapter 2) because the influence of the initial curvature of the beam upon the shear stress is very small ( $\mathrm{Li} 1987$ ).

\subsubsection{Shear Lag}

Shear lag can be accounted for in the same way as in straight box beams. For a biaxially symmetric single-cell curved box beam subjected to symmetric vertical load $q_{y}$ only, as shown in Fig. 4.5, based on Reissner's hypothesis, the longitudinal displacement of the cross section can be approximated as follows

$$
u^{\prime}(x, y, z)=y \bar{\theta}_{x}-\omega_{v}(x, y) \psi^{\prime}(z)=-y v_{0}^{\prime}(z)-\omega_{v}(x, y) \psi^{\prime}(z)
$$

where $\omega_{v}(x, y)$ and $\psi(z)$ have been defined in Eq. (2.3.1). Similar to Eq. (2.3.2), the alternative expression to $\mathrm{Eq} .(4.2 .22)$ may be written as

$$
\omega(s, z)=-\omega(s)_{b} v_{0}^{\prime}(z)-\omega_{v}(s) \psi^{\prime}(z)
$$

Substituting Eq. (4.2.23) into Eq. (4.2.13), the normal strain can be found

$$
\epsilon_{z}=\frac{\partial u(x, y, z)}{\partial z}-\frac{u(x, y, z)}{R_{0}}
$$


or

$$
\epsilon_{z}=-\omega_{b}(s) v_{0}^{\prime \prime}-\omega_{v}^{\prime \prime}(s) v^{\prime \prime}+\chi\left(y-y_{s}\right) \theta_{z}
$$

The shear strain can be determined by Eq. (2.3.3b)

$$
\gamma_{s z}=\frac{\partial u(s, z)}{\partial s}=-\dot{\omega}_{b}(s) v_{0}^{\prime}-\dot{\omega}_{v}(s) \psi^{\prime}
$$

where $\dot{\omega}_{b}(s)=d \omega_{b}(s) / d s$ and $\dot{\omega}_{v}(s)=d \omega_{v}(s) / d s$. The normal and shear stresses are given by

$$
\begin{aligned}
& \sigma_{z}=E \epsilon_{z}=-E\left[\omega_{b}(s) v_{0}^{\prime \prime}+\omega_{v}(s) \psi^{\prime \prime}-\chi\left(y-y_{s}\right) \theta_{z}\right] \\
& \tau_{s z}=G \gamma_{s z}=-G\left[\dot{\omega}_{b}(s)\left(v_{0}^{\prime}+\dot{\omega}_{v}(s) \psi^{\prime}\right]\right.
\end{aligned}
$$

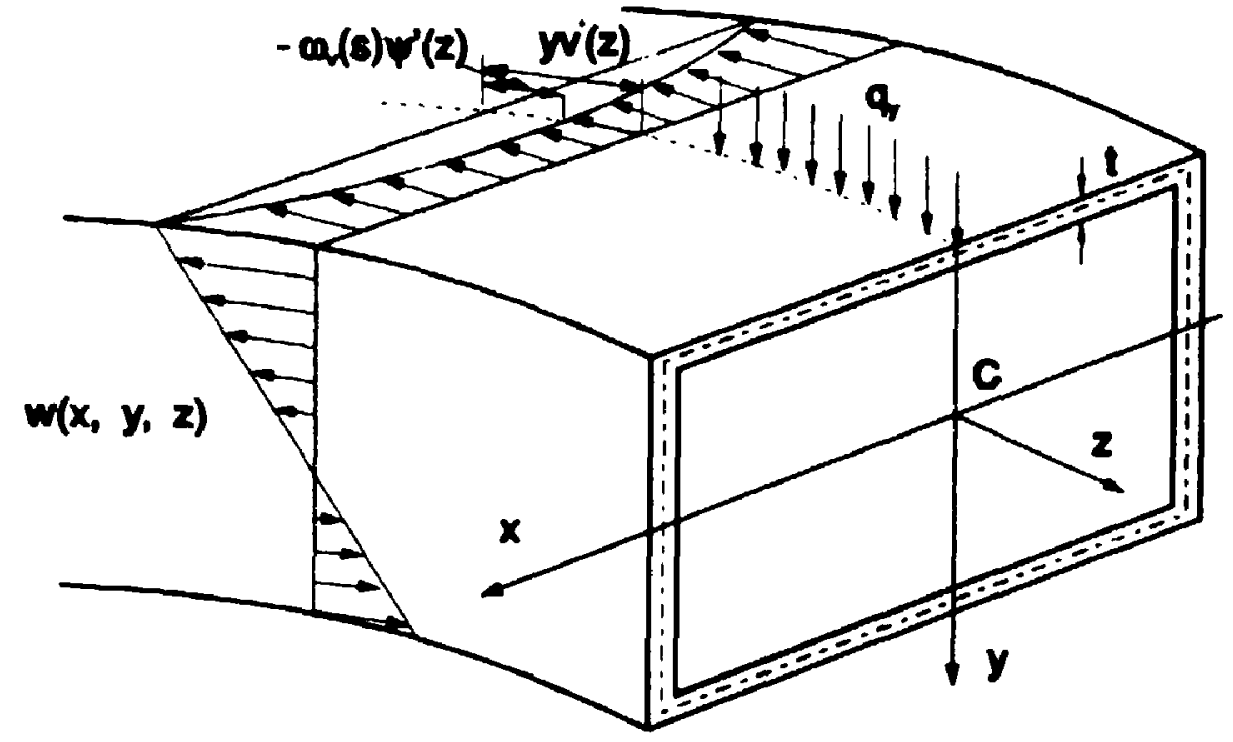

Fig. 4.5 Biaxially symmetric single-cell curved box beam for shear lag analysis.

The governing differential equations of the problem can be developed by the principal of virtual work. The internal virtual work $\delta W$, equals

$$
\delta W_{1}=\int_{V}\left(\sigma_{z} \delta \epsilon_{2}+\tau_{32} \delta \gamma_{32}\right) d v
$$


Substituting Eqs. (4.2.24a) to (4.2.25b) into the above equation, it yields

$$
\begin{aligned}
\delta W_{1}= & \int_{V} E\left[\omega_{b}^{2} v_{0}^{\prime \prime} \delta v_{0}^{\prime \prime}+\omega_{b} \omega_{v} v_{0}^{\prime \prime} \delta \psi^{\prime \prime}-\omega_{b} \chi\left(y-y_{c}\right) v_{0}^{\prime \prime} \delta \theta_{z}+\omega_{v} \omega_{b} \psi^{\prime \prime} \delta v_{0}^{\prime \prime}+\omega_{v}^{2} \psi^{\prime \prime} \delta \psi^{\prime \prime}\right. \\
& -\omega_{v} \chi\left(y-y_{0}\right) \psi^{\prime \prime} \delta \theta_{z}-\chi \omega_{b}\left(y-y_{0}\right) \theta_{z} \delta v_{0}^{\prime \prime}-\chi \omega_{v}\left(y-y_{0}\right) \theta_{z} \delta \psi^{\prime \prime}+ \\
& \left.\chi^{2}\left(y-y_{c}\right)^{2} \theta_{z} \delta \theta_{z}\right] d v+\int_{V} G\left(\dot{\omega}_{b}^{2} v_{0}^{\prime} \delta v_{0}^{\prime}+\dot{\omega}_{b} \dot{\omega}_{v} v_{0}^{\prime} \delta \psi^{\prime}+\dot{\omega}_{b} \dot{\omega}_{v} \psi^{\prime} \delta v_{0}^{\prime}+\dot{\omega}_{v}^{2} \psi^{\prime} \delta \psi^{\prime}\right) d v
\end{aligned}
$$

or

$$
\begin{aligned}
\delta W_{1}= & \int_{0}^{l} E\left[I_{x} v_{0}^{\prime \prime} \delta v_{0}^{\prime \prime}+I_{x v} v_{0}^{\prime \prime} \delta \psi^{\prime \prime}-I_{x x} v_{0}^{\prime \prime} \delta \theta_{z}+I_{v x} \psi^{\prime \prime} \delta v_{0}^{\prime \prime}\right. \\
& \left.+I_{v} \psi^{\prime \prime} \delta \psi^{\prime \prime}-I_{v x} \psi^{\prime \prime} \delta \theta_{z}-I_{x x} \theta_{z} \delta v_{0}^{\prime \prime}-I_{v x} \theta_{z} \delta \psi^{\prime \prime}+I_{x} \theta_{z} \delta \theta_{z}\right] d v \\
& +\int_{0}^{l} G\left(J_{x} v_{0}^{\prime} \delta v_{0}^{\prime}+J_{x v} v_{0}^{\prime} \delta \psi^{\prime}+J_{x v} \psi^{\prime} \delta v_{0}^{\prime}+J_{v} \psi^{\prime} \delta \psi^{\prime}\right) d v
\end{aligned}
$$

where

$$
\begin{aligned}
& I_{x}=\int_{A} \omega_{b}^{2}(s) d A=\int_{A} y^{2} d A \\
& I_{x v}=\int_{A} \omega_{b}(s) \omega_{v}(s) d A \\
& I_{v}=\int_{A} \omega_{v}^{2}(s) d A \\
& J_{x}=\int_{A} \dot{\omega}_{b}^{2}(s) d A \\
& J_{x v}=\int_{A} \dot{\omega}_{b}(s) \dot{\omega}_{v}(s) d A \\
& J_{v}=\int_{A} \dot{\omega}_{v}^{2}(s) d A \\
& I_{x \chi}=\int_{A} \omega_{b}(s) \chi\left(y-y_{s}\right) d A \\
& I_{v \chi}=\int_{A} \omega_{v}(s) \chi\left(y-y_{s}\right) d A \\
& I_{\chi}=\int_{A} \chi^{2}\left(y-y_{s}\right)^{2} d A
\end{aligned}
$$

The external virtual work $\delta W_{e}$ can be written as

$$
\delta W_{e}=\int_{0}^{l} q_{y} \delta v_{0}(z) d z+\sum_{k} P_{y i} \delta v_{0}\left(z_{i}\right)
$$

where $q_{y}$ is the applied distributed load in the $y$ direction and $P_{y i}$ is the applied concentrated load acting at $z=z_{1}$ in the $y$ direction; $k$ is the number of concentrated loads acting on the box beam.

Equating $\delta W_{i}$ and $\delta W_{c}$ and integrating by parts, we obtain the governing differential equations as follows

$$
E I_{x} \frac{d^{4} v_{0}(z)}{d z^{4}}+E I_{x v} \frac{d^{4} \psi(z)}{d z^{4}}-G J_{x} \frac{d^{2} v_{0}(z)}{d z^{2}}-G J_{x v} \frac{d^{2} \psi(z)}{d z^{2}}-E I_{x x} \frac{d^{2} \theta_{z}}{d z^{2}}=q_{y}
$$




$$
\begin{gathered}
E I_{x v} \frac{d^{4} v_{0}(z)}{d z^{4}}+E I_{v} \frac{d^{4} \psi(z)}{d z^{4}}-G J_{x v} \frac{d^{2} v_{0}(z)}{d z^{2}}-G J_{v} \frac{d^{2} \psi^{\prime}(z)}{d z^{2}}-E I_{v \lambda} \frac{d^{2} \theta_{z}}{d z^{2}}=0 \\
-E I_{x_{\lambda}} \frac{d^{2} v_{0}}{d z^{2}}-E I_{v \lambda} \frac{d^{2} \psi}{d z^{2}}+E I_{\lambda} \theta_{z}=0
\end{gathered}
$$

The boundary conditions for Eqs. (4.2.31) dre:

For simply supported end:

$$
\begin{aligned}
& v_{0}=0 \\
& v_{0}^{\prime \prime}=0 \\
& \psi=0 \\
& \psi^{\prime \prime}=0
\end{aligned}
$$

If torsion is restrained by end diaphragms, but the cross section is free to warp out of its plane, we have

$$
\begin{aligned}
\theta_{2} & =0 \\
\theta_{z}^{\prime \prime} & =0
\end{aligned}
$$

For fixed end:

$$
\begin{aligned}
v_{0} & =0 \\
v_{0}^{\prime} & =0 \\
\psi^{\prime} & =0 \\
\psi^{\prime} & =0
\end{aligned}
$$

If torsion and torsional warping are restrained by end diaphraguns, we have

$$
\begin{aligned}
& \theta_{z}=0 \\
& \theta_{z}^{\prime}=0
\end{aligned}
$$

For the more general multicell curved box beams, Roilk and Sedlacek's method for straight box beams, as discussed in Sec. (2.3), will be used to accoumt for shear lag. 

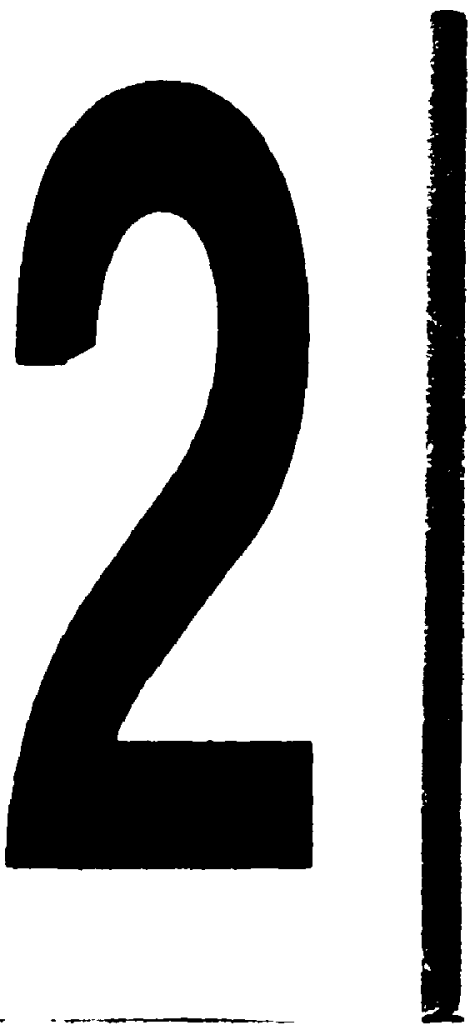

PA-1 34" "x4" PHOTOCRAPHC MICROCOPY TARGET Nas 1010a AMEAlEO we EOUNALENT

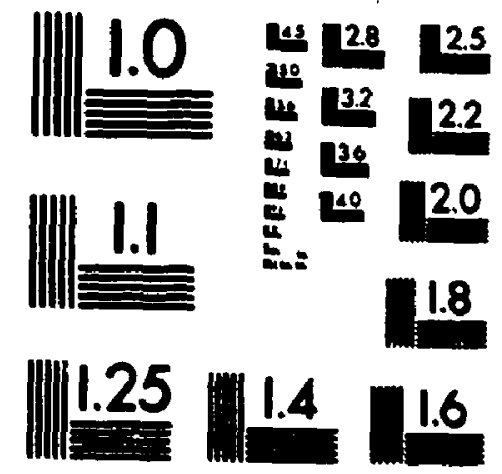

PAECHSIONE RESOLUTION TARCETS

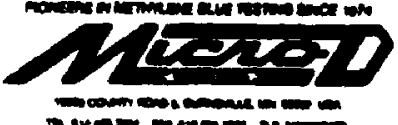




\subsubsection{Distortion}

A single-cell monosymmetric curved box beam subjected to a vertical distributed load $q_{y}$ with an eccentricity $e$ is shown in Fig. 4.6. The displacement field can be describec by the following vector

$$
\{D\}=\left[\begin{array}{lll}
v_{0} & \theta_{z} & \beta
\end{array}\right]^{T}
$$

The displacements $u(x, y, z)$ and $v(x, y, z)$ corresponding to those in Eqs. (4.2.6) and (4.2.7) can be rewritten as

$$
\begin{aligned}
& u(x, y, z)=-\left(y-y_{s}\right) \theta_{z}-\left(y-y_{d}\right) \beta \\
& v(x, y, z)=v_{0}(z)-\left(x-x_{s}\right) \theta_{z}-\left(x-x_{d}\right) \beta
\end{aligned}
$$

where $x_{d}$ and $y_{d}$ are the coordinates of distortion center $D$, while $\beta$ and $\omega_{d}$ were defined by Eq. (2.5.1).

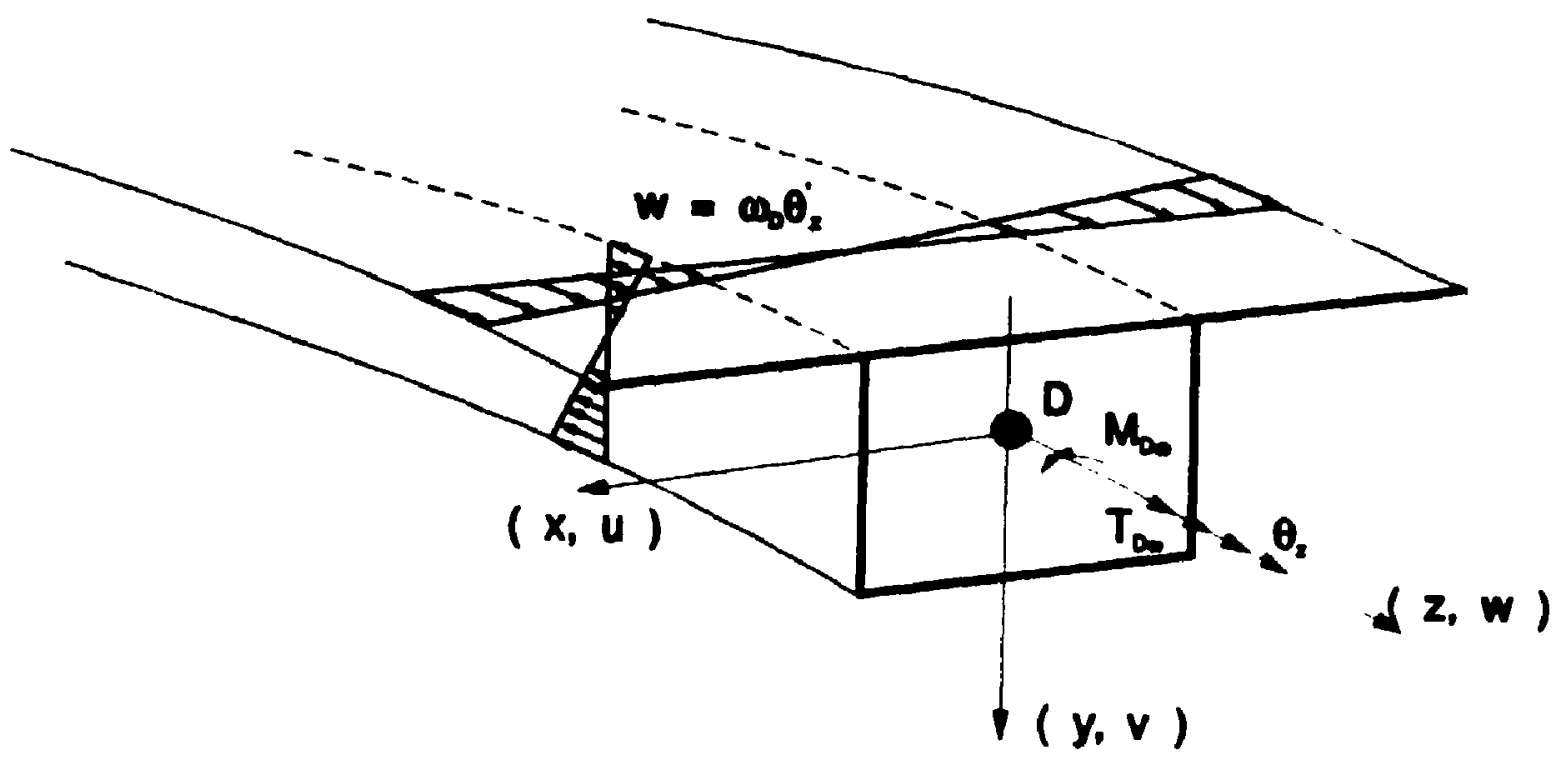

Fig. 4.6 Stress resultants and displacements for distortional analysis.

Similar to the distortional analysis of straight box beams, the longitudinal displacement may be defined as

$$
w(x, y, z)=y \bar{\theta}_{x}-\omega \vec{\theta}_{z}-\omega_{d} \beta^{\prime}
$$


The normal strain is given by

$$
\epsilon_{z}=\frac{\partial w(x, y, z)}{\partial z}-\frac{u(x, y, z)}{R_{0}}
$$

or

$$
\epsilon_{z}=-y v_{0}^{\prime \prime}-\omega\left(\theta_{z}^{\prime \prime}+\chi v_{0}^{\prime \prime}\right)-\omega_{d} \beta^{\prime \prime}+\chi\left(y-y_{d}\right) \theta_{z}+\chi\left(y-y_{d}\right) \beta
$$

which can be expressed in matrix form as follows

$$
\epsilon_{2}=-\{\omega\}^{T}[d]\{D\}
$$

where $\{\omega\}$ is the warping vector and $[d]$ is an operator matrix as follows

$$
\begin{aligned}
& \{\omega\}=\left[\begin{array}{llll}
y & \omega & \omega_{d} & 1
\end{array}\right]^{T} \\
& {[d]=\left[\begin{array}{ccc}
d^{2} & -\chi & -\chi \\
x d^{2} & d^{2} & 0 \\
0 & 0 & d^{2} \\
0 & x y_{s} & x y_{d}
\end{array}\right]}
\end{aligned}
$$

in which $d=d / d z$. Thus, the normai stress $\sigma_{z}$ is

$$
\sigma_{z}=E \epsilon_{z}=-E\{\omega\}^{T}[d]\{D\}
$$

The governing differential equations can be dervied in the same way as for straight box beams. The internal virtual work $\delta W_{i}$ is given by

$$
\begin{aligned}
\delta W_{1} & =\int_{V}\left(\sigma_{z} \delta \epsilon_{z}+\tau_{d} \delta \gamma_{d}+\tau_{s} \delta \gamma_{s}\right) d v+\int_{0}^{l} K_{d} \beta \delta \beta d z \\
& =\int_{0}^{l}\left(\{D\}^{T}[d]^{T}[E I][d] \delta\{D\}+\left(\theta_{z}^{\prime}+\chi v_{0}^{\prime}\right) G J_{s} \delta\left(\theta_{z}^{\prime}+\chi v_{0}^{\prime}\right)\right. \\
& \left.+\beta^{\prime} G J_{d} \delta \beta^{\prime}+\beta E_{s} K_{d} \delta \beta\right) d z
\end{aligned}
$$

where $J_{d}$ and $K_{d}$ were defined by Eqs. (2.5.21a) and (2.5.21b);

$$
[E I]=\int_{A} E\left\{\omega^{\prime}\right\}\{\omega\}^{T} d A=\left[\begin{array}{cccc}
E I_{x} & 0 & 0 & 0 \\
0 & E I_{\omega} & 0 & 0 \\
0 & 0 & E I_{d \omega 0} 0 & \\
0 & 0 & 0 & E A
\end{array}\right]
$$

in which $I_{x}, I_{w}$ and $I_{d w}$ are the moments of inertia with respect to the principal coordinates $y, w$ and $\omega_{d}$, respectively, and $A$ is the area of the cross section. 
The external virtual work $\delta H_{e}$ is

$$
\delta W_{e}=\int_{0}^{l}\left(q_{y} \delta v_{0}+m_{1} \delta\left(\theta_{z}+v_{0}\right)+\frac{m_{t}}{2} \delta \beta\right) d z
$$

where $m_{t}=q_{y} e$. Thus, the governing differential equations for distortional analysis of curved box beam are

$$
[G]\{D\}=\{p\}
$$

where

in which

$$
[G]=\left[\begin{array}{lll}
G_{11} & G_{12} & G_{13} \\
G_{21} & G_{22} & G_{23} \\
G_{31} & G_{32} & G_{33}
\end{array}\right]
$$

$$
\begin{aligned}
& G_{33}=\chi^{2}\left(E I_{x}+y_{d}^{2} A\right)+E I_{w} d^{4}-G J_{s} d^{2}+K_{d} \\
& \{p\}=\left\{\begin{array}{c}
q_{y}+\chi^{m_{t}} \\
m_{t} \\
m_{t} / 2
\end{array}\right\}
\end{aligned}
$$

\subsection{Generalized Vlasov's Theory for Curved Multicell Box Beams}

An improved Vlasov's theory for curved nulticell box beams is developed in this section. The theory will consider all the structural actions considered in the straight box beams. In addition, shear deformations are included and the flexural theory is based on Timoshenko's beam theory, i.e. the rotations $\theta_{x}$ and $\theta_{y}$ about the $x$ and $y$ axes are assumed independent from the lateral displacements. Sedlacek, and Roik and Sedlacek's work described in Chapter 2 will be used for distortional and shear Ing analysis. The interaction between the longitudinal and the transverse deformations in the box will also be included based on the theory of elastirity. 


\section{Coordinate system.}

For non-prismatic curved box beams, the positions of the centroid and shear center of the cross section change from one section to the next along the beam. Thus these points do not have the same significance as in prismatic beams. For the convenience of analysis, the origin $O$ of the coordinate system will be fixed at the middle of the top flange. In addition to the curvilinear coordinates $x, y$ and $z$ for the box beam shown in Fig. 4.7, for each plate comprising the walls of the box a local coordinate system $s, z$ and $n$ is introduced in such a way that the $z$-axis and the beam axis are colinear and the $s$ and $n$ axes are tangent and normal to the midsurface of the plate, respectively.

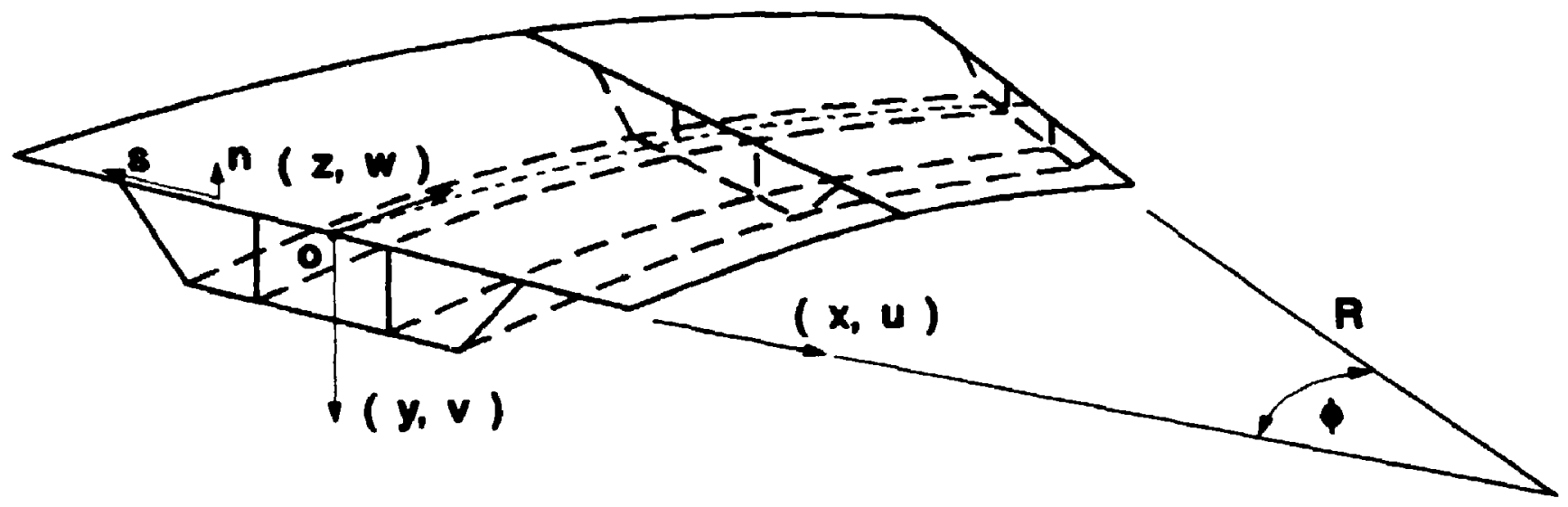

Fig. 4.7 Coordinates for non-prismatic curved box beam.

Displacements, strains and stresses.

The displacements due to extension, flexure, torsion, distortion and shear lag efferts for a curved multicell box beam can be defined as

$$
\{D\}=\left[\begin{array}{llllllllllll}
u_{0} & v_{0} & w_{0} & \theta_{r} & \theta_{y} & \theta_{2} & \beta_{1} & \ldots & \beta_{n} & \psi_{1} & \ldots & \psi_{m}
\end{array}\right]^{T}
$$

where $u_{0}, "{ }^{\prime}$ and $w_{0}$ are the displacements of the reference point $O$ in the $x, y$ and $z$ directions, respectively, $\theta_{z}$ is the angle of twist, and $\theta_{x}$ and $\theta_{y}$ are rotations in the $s$ and $y$ directions, including rotations due to shear deformations. $\beta_{1}$ to $\beta_{n}$ and $\psi_{1}$ 
to $v^{\prime} m$ are the same as defined in Eq. (2.6.1). Based on the generalized Vlasur's thin-walled beam theory, the displacement components at the midsurface of a given plate comprising the cross section shown in Fig. 4.8 are given by

$$
\begin{aligned}
& w(s, z)=\left\{\Phi_{1}(s)\right\}^{T}\{D\}+\left\{\Phi_{2}(s)\right\}^{T}\left\{D^{\prime}\right\} \\
& v_{s}(s, z)=\{\Psi(s)\}^{T}\{D\} \\
& v_{n}(s, z)=\{\equiv(s)\}^{T}\{D\}
\end{aligned}
$$

where

$$
\begin{aligned}
& \left\{\Phi_{1}(s)\right\}=\left[\begin{array}{llllllll}
0 & 0 & (1-x \lambda) & \left(y+w_{\lambda}\right) & -x & 0 & \ldots & 0
\end{array}\right]^{T} \\
& \left\{\Phi_{2}(s)\right\}=\left[\begin{array}{lllllllllll}
0 & \ldots & 0 & -\omega & -\omega_{d 1} & \ldots & -\omega_{d n} & -\omega_{n 1} & \ldots & -\omega_{n m}
\end{array}\right]^{T} \\
& \{\Psi(s)\}=\left[\begin{array}{lllllllllllll}
\Psi_{1} & \Psi_{2} & 0 & 0 & 0 & \rho(s) & 0 & \Psi_{d 1} & \ldots & \Psi_{d n} & 0 & \ldots & 0
\end{array}\right]^{T} \\
& \{\equiv(s)\}=\left[\begin{array}{llllllllllllll}
\Xi_{1} & \Xi_{2} & 0 & 0 & 0 & \Xi_{6} & 0 & \Xi_{d 1} & \ldots & \Xi_{d n} & 0 & \ldots & 0
\end{array}\right]^{T}
\end{aligned}
$$

in which $\omega, \omega_{d 1}$ to $\omega_{d n}$ and $\omega_{v 1}$ to $\omega_{v m}$ are the same as defined in Eq. (2.6.2), $\{\Psi(s)\}$ and $\{\equiv(s)\}$ are the displacement shape functions for $v_{s}(s, z)$ and $v_{n}(s, z)$ due to unit $\{D\}, \rho(s)$ is the perpendicular distance from origin $O$ to the tangent at the middle surface of the plate. The quantities $\Psi_{1}, \Psi_{2}, \Psi_{d 1}$ to $\Psi_{d n}, \Xi_{1}, \Xi_{2}, \Xi_{6}$, and $\Xi_{d 1}$ to $\Xi_{d n}$ are illustrated in Fig. 4.9.

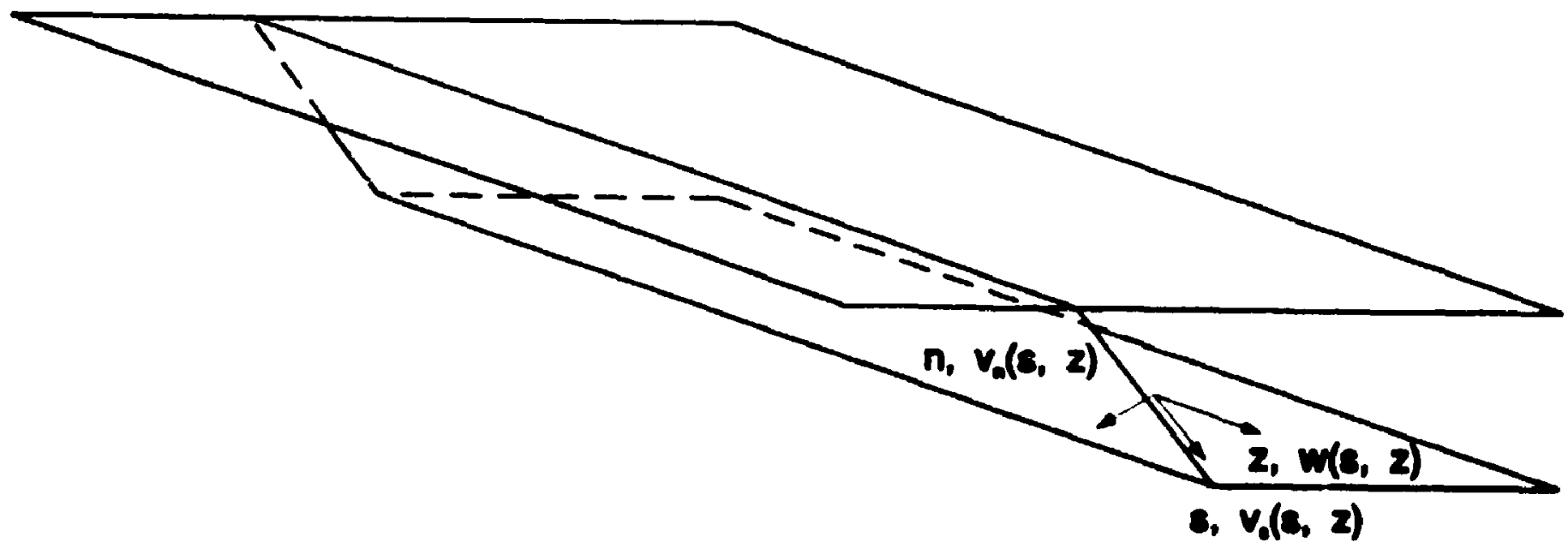

Fig. 4.8 Displacements at midsurface of the cross section.

Considering the plate bending in the transverse direction, i.e. normal to the plate, the displacement components of an arbitrary point of the cross section are 


$$
\begin{aligned}
& u(s, z, n)=w(s, z)-n \frac{\partial v_{n}(s, z)}{\partial z} \\
& v_{s}(s, z, n)=v_{s}(s, z)-n \frac{\partial v_{n}(s, z)}{\partial s} \\
& v_{n}(s, z, n)=v_{n}(s, z)
\end{aligned}
$$

Substituting Eqs. (4.3.2a) to (4.3.2c) into Eqs. (4.3.4a) to (4.3.4.c), the following matrix equations can be found

$$
\left\{\begin{array}{l}
w(s, z, n) \\
v_{1}(s, z, n) \\
v_{n}(s, z, n)
\end{array}\right\}=\left\{A_{1}\right\}\{D\}+\left\{A_{2}\right\}\left\{D^{\prime}\right\}
$$

where

$$
\begin{aligned}
& \left\{A_{1}\right\}=\left\{\begin{array}{c}
\left\{\Phi_{1}(s)\right\}^{T} \\
\{\Psi(s)\}^{T}-n\{\dot{\Xi}(s)\}^{T} \\
\{\Xi(s)\}^{T}
\end{array}\right\} \\
& \left\{A_{2}\right\}=\left\{\begin{array}{c}
\left\{\Phi_{2}(s)\right\}^{T}-n\{\Xi(s)\}^{T} \\
0 \\
0
\end{array}\right\}
\end{aligned}
$$

Based on the small deformation theory of linear elasticity, the strain components may be written as

$$
\begin{aligned}
& \epsilon_{z}=\frac{\partial w(s, z, n)}{\partial z}=\frac{\partial w(s, z)}{\partial z}-n \frac{\partial^{2} v_{\mathfrak{n}}(s, z)}{\partial z^{2}} \\
& \epsilon_{s}=\frac{\partial v_{g}(s, z)}{\partial s}-n \frac{\partial^{2} v_{n}(s, z)}{\partial s^{2}} \\
& \gamma_{s z}=\frac{\partial w(s, z)}{\partial s}+\frac{\partial v_{s}(s, z)}{\partial z}-2 n \frac{\partial^{2} v_{n}(s, z)}{\partial z \partial s}
\end{aligned}
$$

Substituting Eqs. (4.3.5a) to (4.3.5c) into Eqs. (4.3.6a) to (4.3.6c), the strain vector can be obtained

$$
\left\{\begin{array}{c}
\epsilon_{2} \\
\epsilon_{s} \\
\gamma_{s 2}
\end{array}\right\}=\left[B_{1}\right]\{D\}+\left[B_{2}\right]\left\{D^{\prime}\right\}+\left[B_{3}\right]\left\{D^{\prime \prime}\right\}
$$

where

$$
\begin{gathered}
{\left[B_{1}\right]=\left\{\begin{array}{c}
0 \\
\{\dot{\Psi}(s)\}^{T}-n\{\dot{\Xi}(s)\}^{T} \\
\left\{\dot{\Phi}_{1}(s)\right\}^{T}
\end{array}\right\}} \\
{\left[B_{2}\right]=\left\{\begin{array}{c}
\left\{\Phi_{1}(s)\right\}^{T} \\
0 \\
\left\{\dot{\Phi}_{2}(s)\right\}^{T}+\{\Psi(s)\}^{T}-2 n\{\dot{\Xi}(s)\}^{T}
\end{array}\right\}} \\
{\left[B_{3}\right]=\left\{\begin{array}{c}
\left\{\Phi_{2}(s)\right\}^{T}-n\{\Xi(s)\}^{T} \\
0 \\
0
\end{array}\right\}}
\end{gathered}
$$


The stress-strain relationship is given by

$$
\{\sigma\}=[E]\{\epsilon\}=[E]\left(\left[B_{1}\right]\{D\}+\left[B_{2}\right]\left\{D^{\prime}\right\}+\left[B_{3}\right]\left\{D^{\prime \prime}\right\}\right)
$$

where

$$
\begin{aligned}
& \{\sigma\}=\left[\begin{array}{lll}
\sigma_{2} & \sigma_{\theta} & T_{a z}
\end{array}\right]^{T} \\
& {[E]=\frac{1}{1-\nu_{2} \nu_{2}}\left[\begin{array}{ccc}
E_{z} & y_{2} E_{z} & 0 \\
\nu_{2} E_{2} & E_{2} & 0 \\
0 & 0 & \left(1-\nu_{2} \nu_{2}\right) G
\end{array}\right]}
\end{aligned}
$$

where $E_{z}$ and $E_{z}$ are Young's moduli in the longitudinal and transverse directions respectively; $\nu_{z}$ and $\nu_{z}$ are the Poisson's ratios in the $z$ and $s$ directions, and $\nu_{z} E_{z}=$ $\nu_{s} E_{s}$.

\section{Governing differential equation.}

The governing differential equation and the boundary conditions can be derived by the principle of virtual work. From Eqs. (4.3.7) and (4.3.9), the internal virtual work $\delta W$, can be found as follows

$$
\begin{aligned}
\delta W_{\imath} & =\int_{V}\{\sigma\}^{T} \delta\{\epsilon\} d v \\
& =\int_{V}\left(\{D\}^{T}\left[B_{1}\right]^{T}+\left\{D^{\prime}\right\}^{T}\left[B_{2}\right]^{T}+\left\{D^{\prime \prime}\right\}^{T}\left[B_{3}\right]^{T}\right)[E] \\
& \delta\left(\left[B_{1}\right]\{D\}+\left[B_{2}\right]\left\{D^{\prime}\right\}+\left[B_{3}\right]\left\{D^{\prime \prime}\right\}\right) d v
\end{aligned}
$$

Define

$$
\left[C_{1}\right]=\int_{A}\left[B_{1}\right]^{T}[E][B,] d A \quad(i, j=1,2,3)
$$

and after partial integration, Eq. (4.3.12) becomes

$$
\begin{aligned}
\delta W_{1} & =\int\left(\{D\}^{T}\left[C_{11}\right]-\left\{D^{\prime \prime}\right\}^{T}\left[C_{22}\right]+\left\{D^{I V}\right\}^{T}\left[C_{33}\right]\right) \delta\{D\} d x \\
& +\left[\left(\left\{D^{\prime}\right\}^{T}\left[C_{23}\right]+\left\{D^{\prime \prime}\right\}^{T}\left[C_{33}\right]\right) \delta\left\{D^{\prime}\right\}\right]_{0}^{l} \\
& +\left[\left(\{D\}^{T}\left[C_{12}\right]+\left\{D^{\prime}\right\}^{T}\left[C_{22}\right]-\left\{D^{\prime \prime}\right\}^{T}\left[C_{33}\right]\right) \delta\{D\}\right]_{0}^{l}
\end{aligned}
$$

The external virtual work $\delta W_{e}$ is given by

$$
\begin{aligned}
\delta W_{e} & =\int_{V}\{F\}^{T} \delta\left(\left\{A_{1}\right\}\{D\}+\left\{A_{2}\right\}\left\{D^{\prime}\right\}\right) d v \\
& =\int\{p\}^{T} \delta\{D\} d x+\left[\{q\}^{T} \delta\{D\}\right]_{0}^{l}
\end{aligned}
$$

where $\{F\}=\left[\begin{array}{lll}F_{\mathrm{a}} & F_{2} & F_{\mathrm{n}}\end{array}\right]^{T}$ is the external load vector over the whole box beam

and

$$
\{p\}=\int_{A}\left(\left(A_{1}\right\}^{T}\{F\}-\left\{A_{2}\right\}\left\{F^{\prime}\right\}\right) d A
$$



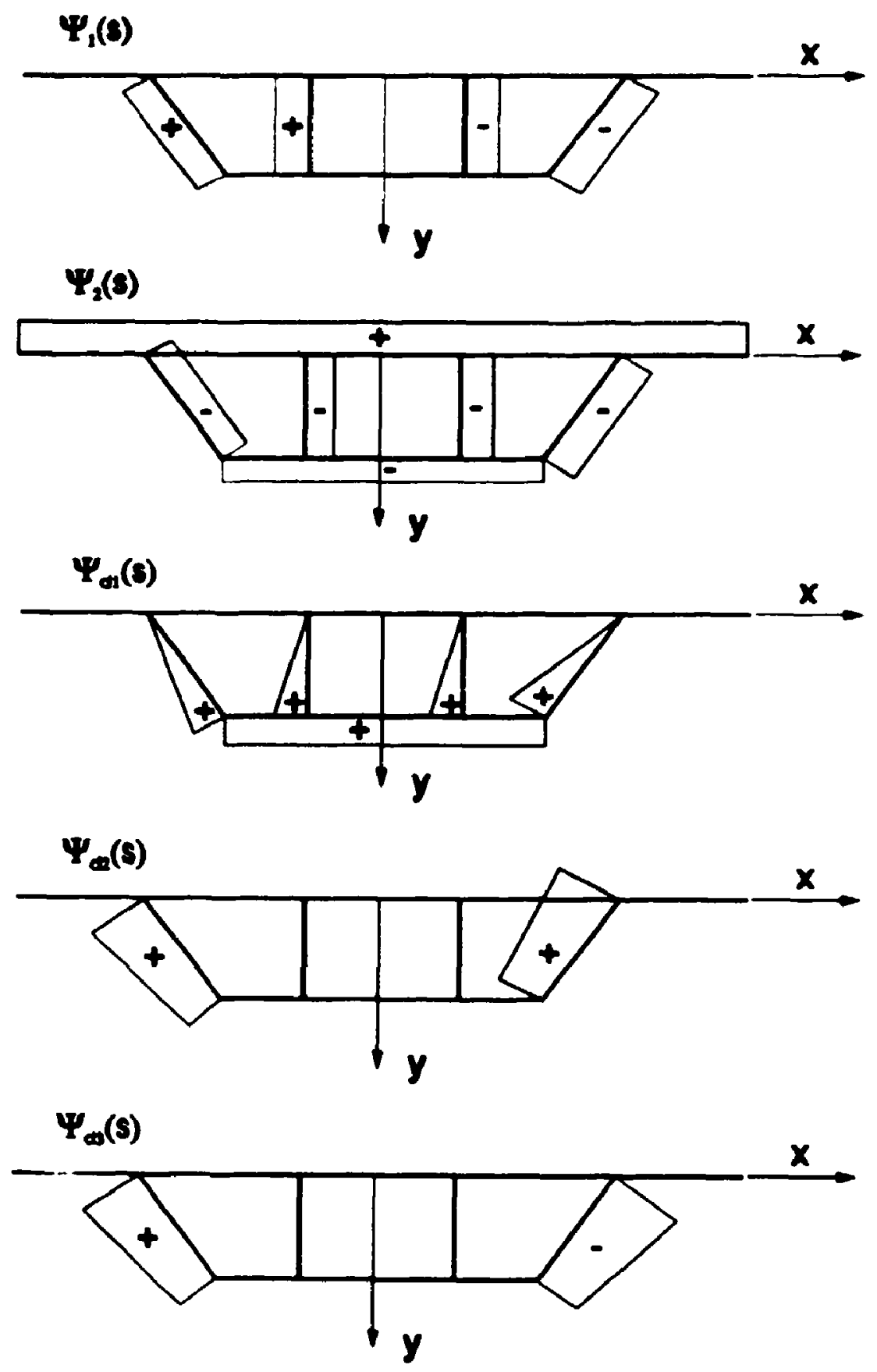

Fig. 4.9 (a) Transverse displacement functions $\Psi(s)$. 

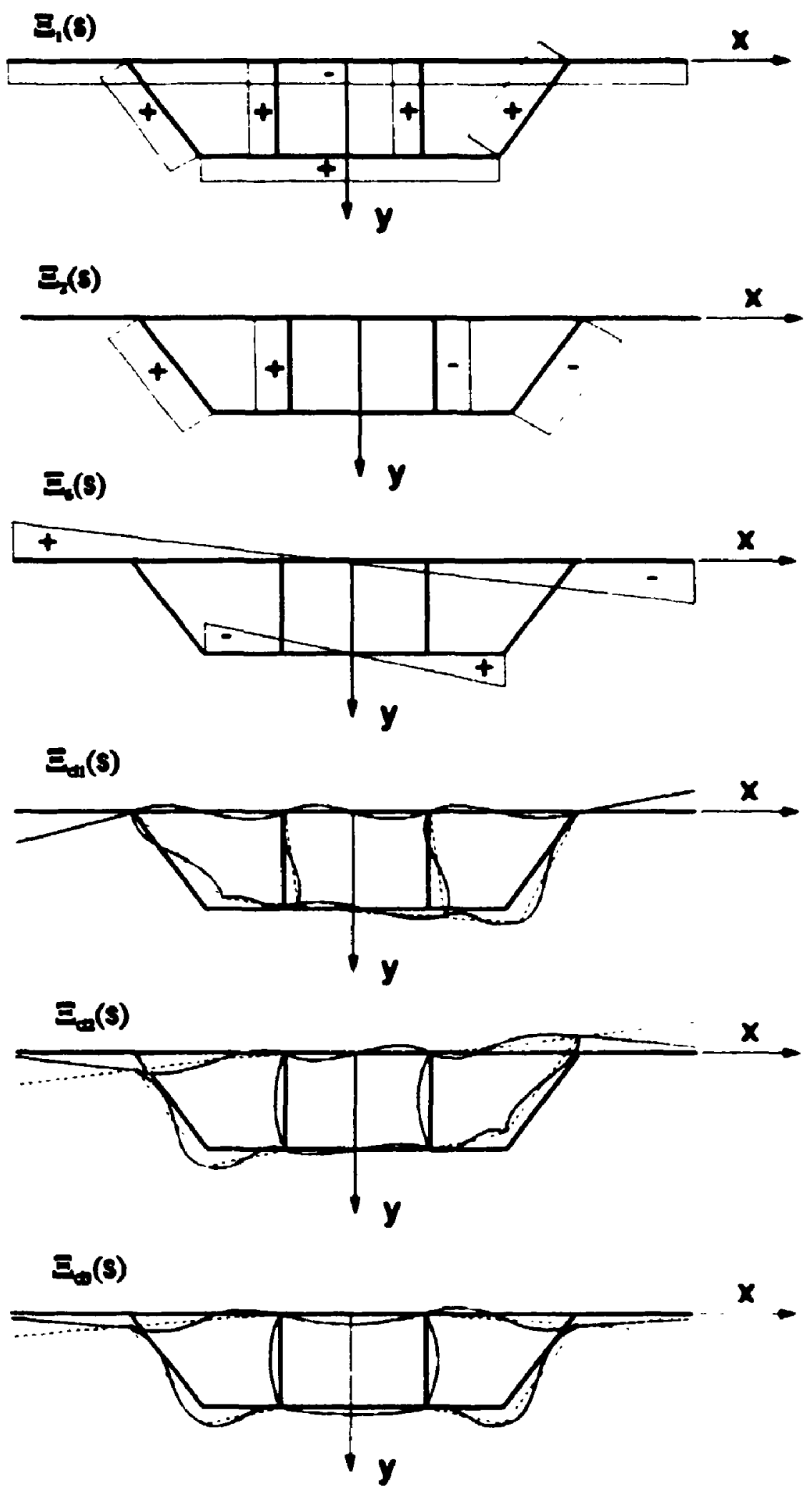

Fig. 4.8 (b) Transverse displacement functions $\Xi(s)$. 


$$
\{q\}=\int_{A}\left\{A_{2}\right\}^{T}\{F\} d A
$$

The governing differential equation can be derived, analogous to the straight box girder in Chapter 2, by means of the calculus of variations. Equating $\delta W$, and $\delta W_{e}$ given in Eqs. (4.3.14) and (4.3.15), and then comparing each term on both sides of the equation, the following system of equations of equilibrium and boundary conditions are obtained:

$$
\begin{gathered}
{\left[C_{33}\right]\left\{D^{I V}\right\}-\left\{C_{22}\right]\left\{D^{\prime \prime}\right\}+\left[C_{11}\right]\{D\}=\{p\}} \\
{\left[\left(\left\{D^{\prime}\right\}^{T}\left[C_{23}\right]+\left\{D^{\prime \prime}\right\}^{T}\left[C_{33}\right]\right) \delta\left\{D^{\prime}\right\}\right]_{0}^{l}=0} \\
\left.\left[\left(\{D\}^{T}\left[C_{12}\right]+\left\{D^{\prime}\right\}^{T}\left[C_{22}\right]-\left\{D^{\prime \prime}\right\}^{T}\left[C_{33}\right]-\{q\}^{T}\right) \delta\{D\}\right\}\right]_{0}^{l}=0
\end{gathered}
$$

It can be seen that Eqs. (4.3.18), (4.3.19) and (4.3.20) represent a highly coupled system of equations, and it is not possible to find the exact solutions for those equations. We will therefore use conventional polynomial displacement functions in order to derive the desired curved multicell box beam element.

\subsection{Finite Element Formulation}

In this section, a finite element model for curved multicell box beam with variable cross sections will be developed based on the theory described in the previous section.

\subsubsection{Element Geometry and Coordinate System}

The geometry of a curved multicell box beam element is shown in Fig. 4.10. The element coordinate system is the same as defined in Fig. 4.7. The element has three nodes along its length. with each node having as many DOF as necessary to describe the box deformations in accordance with the displacement vector given by Eq. (4.3.1).

The element geometry will be described by means of mapping functions. The mapped image of a parent element is shown in Fig. 4.11 and the coordinate mapping functions are given as follows (Bazant and El Nimeiri 1974): 
92

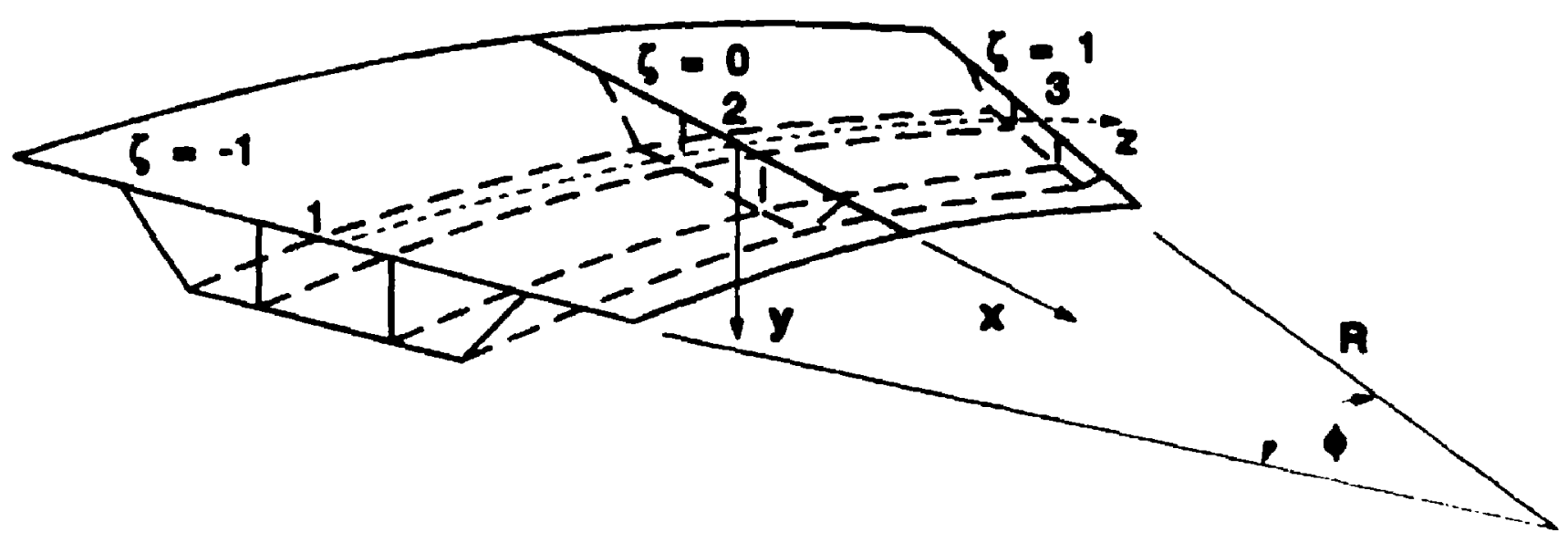

(a) Curved multicell box beam element

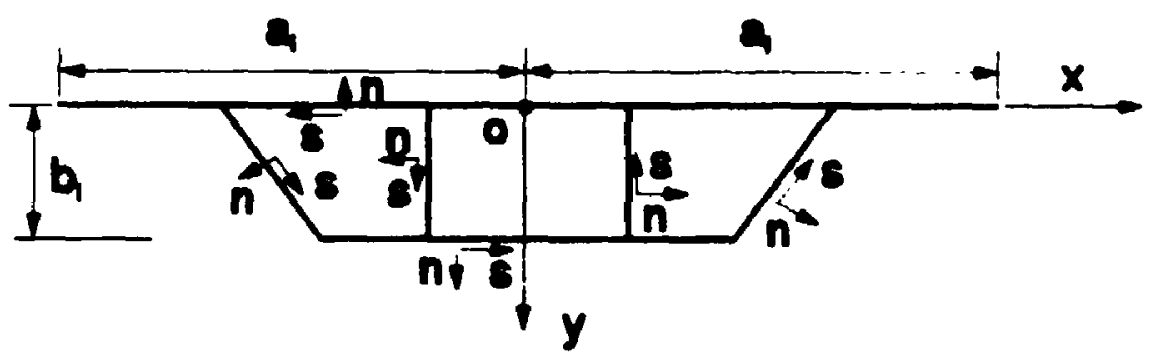

(b) Multicell box cross section

Fig. 4.10 Geometry of curved multicell box beam element.

$$
\begin{aligned}
x= & \frac{1}{2}\left(\zeta^{2}-\zeta\right)\left(\xi a_{x 1}+\eta b_{z 1}\right)+\left(1-\zeta^{2}\right)\left(\xi a_{x 2}+\eta b_{z, 3}\right)+ \\
& \frac{1}{2}\left(\zeta^{2}+\zeta\right)\left(\xi a_{z 3}+\eta b_{z 3}\right) \\
y= & \frac{1}{2}\left(\zeta^{2}-\zeta\right)\left(\xi a_{y 1}+\eta b_{y 1}\right)+\left(1-\zeta^{2}\right)\left(\xi a_{y 2}+\eta b_{y 2}\right)+ \\
& \frac{1}{2}\left(\zeta^{2}+\zeta\right)\left(\xi a_{y 3}+\eta b_{y 3}\right) \\
z= & \frac{1}{2}\left(\zeta^{2}-\zeta\right)\left(\frac{-l}{2}+\xi a_{z 1}+\eta b_{z 1}\right)+\left(1-\zeta^{2}\right)\left(\xi a_{z 2}+\eta b_{z 2}\right)+ \\
& \frac{1}{2}\left(\zeta^{2}+\zeta\right)\left(\frac{1}{2}+\xi a_{z 3}+\eta b_{z 3}\right)
\end{aligned}
$$

where $x, y$ and $z$ are the element curvilinear coordinates; $\xi, \eta$ and $\zeta$ are the coordinates of the parent element that map into $x, y$ and $z$; subscript $i,(i=1,2,3)$, refers to the nodes of the element; $a_{2 i}, a_{y i}, a_{21}$ and $b_{21}, b_{y 1}, b_{21}(i=1,2,3)$ are components 


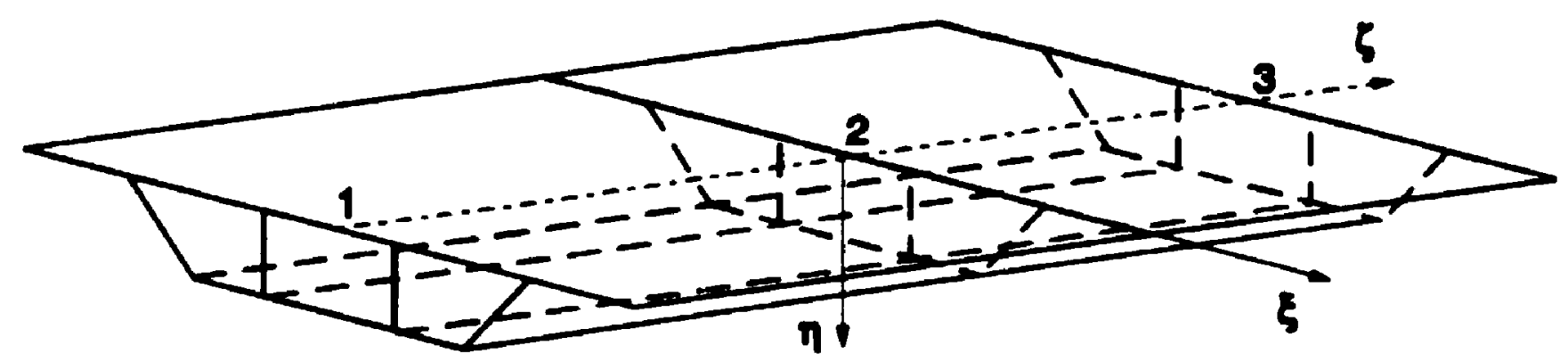

(a) Mapping element

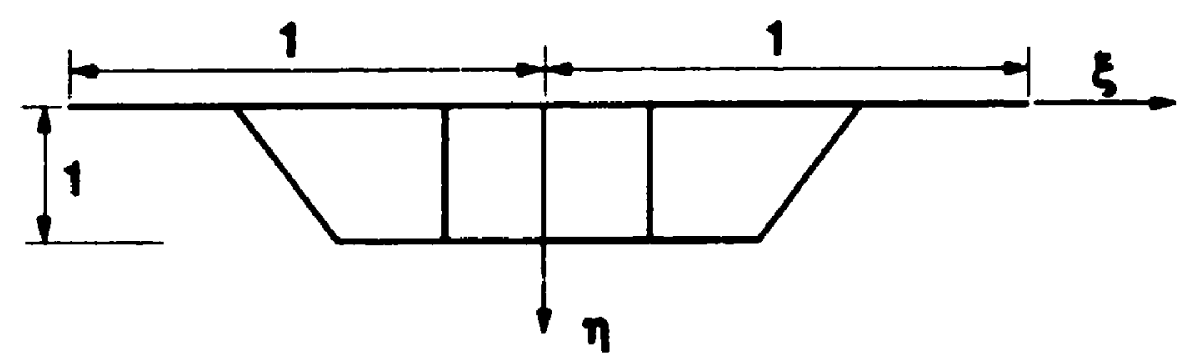

(b) Crose - section

Fig. 4.11 Geometry of mapping element.

of vectors $\{a\}_{\text {, and }}\{b\}_{i}$ and are used to describe the cross sectional geometries at the nodes; the magnitude of these vectors is given by $\left|\{a\}_{i}\right|=a_{1}$ and $\left|\{b\}_{i}\right|=b_{i}$ which, as shown in Fig. 4.10, repesent half the width and the depth, respectively, measured from the origin of the $x$ and $y$ axes at nodal sections.

The mapping functions in Eqs. (4.4.1) are introduced in such a way that $\zeta=$ $-1,0,+1$ are the nodal cross sections; $\xi= \pm 1$ are the extremities of the top slab, and $\eta=0,+1$ are the top and bottom of the box. For the overhanging flanges of length $f$, the limits of $\xi$ at the position of flanges are $\xi_{2}=1+f / a$ and $\xi_{1}=-\xi_{2}$. A parabolic variation of the cross section along the element longitudinal axis can be modelled by appropriate differences of $a_{z 1}$ and $b_{y y}$.

Some common types of box girders are represented as follows. When $a_{z i}=0$ and 
$b_{z i}=0$, the ends of the element are normal to the curved beam axis, and the element is referred to as a right curved box beam element. When $a_{x 1}$ and $b_{y 1}$ are constant. the cross-section of the element will be constant along the longitudinal direction. and the element will be prismatic.

\subsubsection{Shape Functions}

The complete vector of element nodal degrees of freedom is given by the following vector

$$
\begin{aligned}
& \{D\}^{e}=\left[\begin{array}{lllllllllllll}
u_{1} & u_{2} & u_{3} & v_{1} & v_{2} & v_{3} & w_{1} & w_{2} & w_{3} & \theta_{x 1} & \theta_{x 2} & \theta_{x 3}
\end{array}\right. \\
& \begin{array}{llllllllll}
\theta_{y 1} & \theta_{12} & \theta_{y 3} & \theta_{21} & \theta_{z 1}^{\prime} & \theta_{22} & \theta_{x 2}^{\prime} & \theta_{23} & \theta_{z 3}^{\prime}
\end{array} \\
& \begin{array}{lllllllllllll}
\beta_{11} & \beta_{11}^{\prime} & \beta_{12} & \beta_{12}^{\prime} & \beta_{13} & \beta_{13}^{\prime} & \ldots & \beta_{n 1} & \beta_{n 1}^{\prime} & \beta_{n 2} & \beta_{n 2}^{\prime} & \beta_{n 3} & \beta_{n 3}^{\prime}
\end{array} \\
& \left.\begin{array}{lllllllllllllll}
\psi_{11} & \psi_{11}^{\prime} & \psi_{12} & \psi_{12}^{\prime} & \psi_{13} & \psi_{13}^{\prime} & \ldots & \psi_{m 1} & \psi_{m 1}^{\prime} & \psi_{m 2} & \psi_{m 2}^{\prime} & \psi_{m 3} & \psi_{m 3}^{\prime}
\end{array}\right]^{T}
\end{aligned}
$$

The element displacement fields, $\{D\}$, can be related to its nodal displacements, $\{D\}^{e}$, by

$$
\{D\}=\{N]\{D\}^{e}
$$

where $[N]$ is the shape function matrix. The matrix $[N]$ for this element has the form

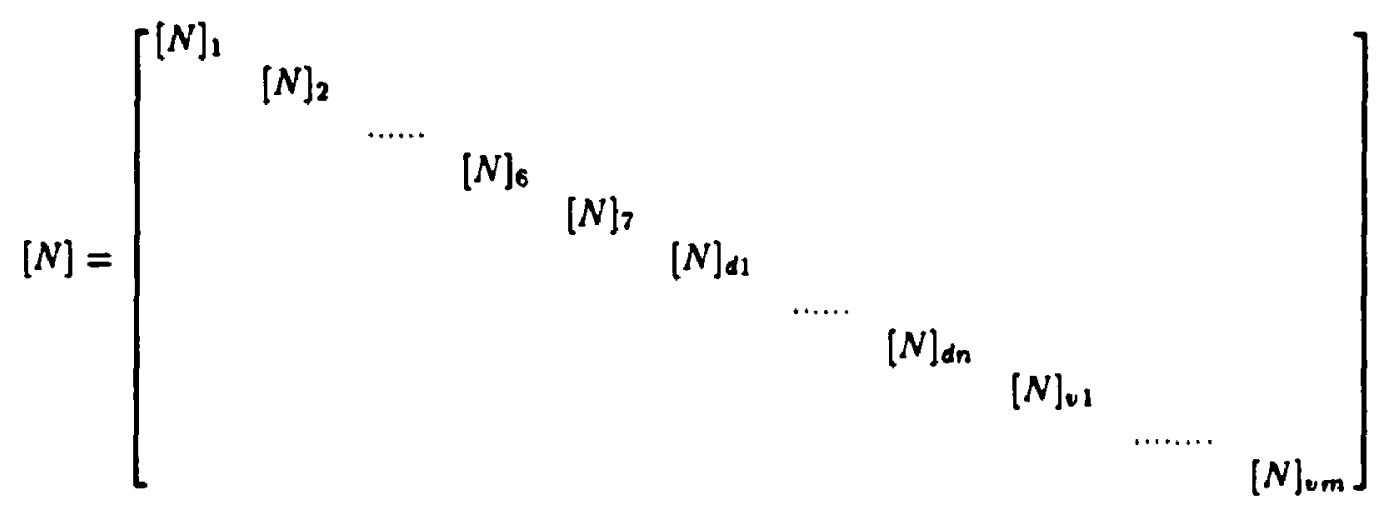

in whicn $\quad[N\}_{i}=\left[\begin{array}{lll}N_{1} & N_{2} & N_{3}\end{array}\right] \quad(i=1$ to 6$)$;

$$
\{N]_{i}=\left[\begin{array}{llllll}
N_{11} & N_{12} & N_{21} & N_{12} & N_{31} & N_{32}
\end{array}\right] \quad(i=7, \text { d1 to } v m) \text {. }
$$

Be use the governing differential equations of curved box beam element can not be solved exactly, polynomial shape functions will be used. In the Timoshenko beam theory, for extensional and flexural effects, $C_{0}$ continuity is required and thus parabolic shape functions are used. 


$$
\begin{aligned}
& N_{1}=\frac{1}{2}\left(\zeta^{2}+\zeta_{0}\right) \quad(i=1,3) \\
& N_{1}=(1 \quad .2) \quad(i=2)
\end{aligned}
$$

where $\zeta_{0}=\zeta \zeta_{1}$. For torsic: $: 1$, distortional and shear lag effects, $C_{1}$ continuity is required, consequently, the following shape functions are used:

$$
\begin{array}{ll}
N_{11}=\frac{\zeta^{2}}{4}\left(4+5 \zeta_{0}-2 \zeta^{2}-3 \zeta_{0}^{3}\right) & (i=1,3) \\
N_{12}=\frac{J_{1}}{4} \zeta^{2}\left(1+\zeta_{0}\right)\left(1-\zeta^{2}\right) & \\
N_{11}=\left(1-\zeta^{2}\right)^{2} & (i=2) \\
N_{i 2} & =J_{1} \zeta\left(1-\zeta^{2}\right)^{2}
\end{array}
$$

where $J_{1}$ is the determinant of the Jacobian matrix $[J]$

$$
[J]=\left[\begin{array}{lll}
\frac{\partial x}{\partial \xi} & \frac{\partial x}{\partial \eta} & \frac{\partial x}{\partial \zeta} \\
\frac{\partial y}{\partial \xi} & \frac{\partial y}{\partial \eta} & \frac{\partial y}{\partial \zeta} \\
\frac{\partial z}{\partial \xi} & \frac{\partial z}{\partial \eta} & \frac{\partial z}{\partial \zeta}
\end{array}\right]
$$

\subsubsection{Stiffness Matrix and Equivalent Nodal Loads}

Substituting Eq. (4.4.3) into Eq. (4.3.7), the strain $\{\epsilon\}$ becomes

$$
\{\epsilon\}=[B]\{D\}^{e}
$$

where $[B]$ is the strain-displacement matrix

$$
[B]=\left\{\begin{array}{c}
\left\{\Phi_{1}\right\}^{T}\left[N^{\prime}\right]+\left(\left\{\Phi_{2}\right\}^{T}-n\{\equiv\}^{T}\right)\left[N^{\prime \prime}\right] \\
-n\{\dot{\Xi}\}^{T}[N] \\
\left\{\dot{\Phi}_{1}\right\}^{T}[N]+\left(\left\{\dot{\Phi}_{2}\right\}^{T}+\{\Psi\}^{T}-2 n\{\dot{\Xi}\}^{T}\right)\left[N^{\prime}\right]
\end{array}\right\}
$$

The stress vector follows as usually

$$
\{\sigma\}=[E]\{\epsilon\}=[E][B]\{D\}^{e}
$$

Thus, the element stiffness matrix is given by

$$
[S]^{C}=\int_{V}[B]^{T}[E][B] d v=\int_{-1}^{+1} \int_{0}^{+1} \int_{-1}^{+1}[B]^{T}[E][B]|J| d \xi d \eta d \zeta
$$

The integration of Eq. (4.4.10) can be achieved by Gauss numerical integration. In the plane of the cross section, three Gauss points are used for each plate segment, 
while two Gauss points are used along the element axis. This is found to eliminate the spurious shear effects usually associated with beam and shell formulations which include shear deformations. Spurious shear effects relative to the torsional, distortional warping and shear lag degrees of freedom are also believed to be eliminated by this reduced order of integration (Bazant and El Nimeiri 1974, Choudhury 1986).

The equivalent nodal load vector is given by

$$
\begin{aligned}
\{F\}^{e}= & \int_{V}\left([N]^{T}\left\{A_{1}\right\}^{T}+\left[N^{\prime}\right]^{T}\left\{A_{2}\right\}^{T}\right)\{F\} d v \\
& \int_{S}\left([N]^{T}\left\{A_{1}\right\}^{T}+\left[N^{\prime}\right]^{T}\left\{A_{2}\right\}^{T}\right)\{T\} d S \\
& \sum\left([N]^{T}\left\{A_{1}\right\}^{T}+\left[N^{\prime}\right]^{T}\left\{A_{2}\right\}^{T}\right)\{P\}
\end{aligned}
$$

where $\{F\}=\left[\begin{array}{lll}F_{x} & F_{s} & F_{n}\end{array}\right]^{T}$ and $\{T\}=\left[\begin{array}{lll}T_{x} & T, & T_{n}\end{array}\right]^{T}$ are the body load vectcr and surface load vector, respectively; $\{P\}=\left[\begin{array}{lll}P_{x} & P_{s} & P_{n}\end{array}\right]^{T}$ is the applied concentrated loads vector. After obtaining the element stiffness matrix and the equivalent nodal load vector, static condensation technique is used to eliminate the internal degrees of freedom at node 2.

\subsubsection{Boundary Conditions}

The boundary conditions for conventional supjort conditions in bridge construction are as follows:

If the displacements at ends of the longitudinal axis of the element are rerstrained, we have:

$$
u_{0}=v_{0}=w_{0}=\psi_{v 1} \ldots=\psi_{v m}=0
$$

If the flexural rotations are restrained,

$$
\theta_{x}=\theta_{y}=\psi_{v 1}^{\prime}=\ldots=\psi_{v m}^{\prime}=0
$$

If torsion and distortion of the cross section are restrained by the end diaphragms, the boundary conditions are

$$
\theta_{2}=\beta_{d 1}=\ldots=\beta_{d n}=0
$$

If torsional, distortional and shear lag warping is restrained, we have:

$$
\theta_{z}^{\prime}=\beta_{d 1}^{\prime}=\ldots=\psi_{v m}^{\prime}=0
$$

If intermediate interior elastic diaphragms are present, the distortional stiffness 
of the diaphragm can be included in the structure stiffness matrix by the same way as discussed in Sec. (2.5.3).

The above derivation completes the theoretical development of a generalized curved multicell box beam element. The accuracy of the element will be tested by the numerical examples in Chapter 6.

\subsection{Multicell Box Girder Assemblages}

Multicell multi-branch box girder assemblages are sometimes used in modern bridge construction. Situations in which this may occur are when a wide lane bridge branches off to two or more narrow lane bridges or when the exit ramps of a wide bridge are an integral part of its superstructure. Through the transformation of the coordinate system, the two finite element models developed in this chapter and in the last chapter, respectively, can be combined to analyze these kind of structures. Consider the box assemblage in Fig. 4.12, the structure can be mod slled by curved box elements for curved ramps and straight box elements for the straight part outside of the intersection. In the intersection region, rigid arms will be used to model this locally stiff area. The actual state of stresses in this zone may be rather complex and would need detailed finite element analysis using shell elements.

The overall or global stiffness matrix can be built up from the individual box girder element stiffness matrices based on the compatibility requirements at the joints of the members. The compatibility conditions of the joints connecting the individual box girder elements are dependent on the type of connection. Figure 4.13 shows some situations which may occur in bridges. In Fig. 4.13(a), elements 1 and 2 have the same cross section and there is complete structural and geometric continuity at the element intersection; thus compatibility should be satisfied for all the nodal degrees of freedom. Since the structural global coordinate system is coincident with the element local coordinate system, no coordinate transformation is needed. Figure 4.13(b) illustrates the case of two elements intersecting at an angle. In this case, we 


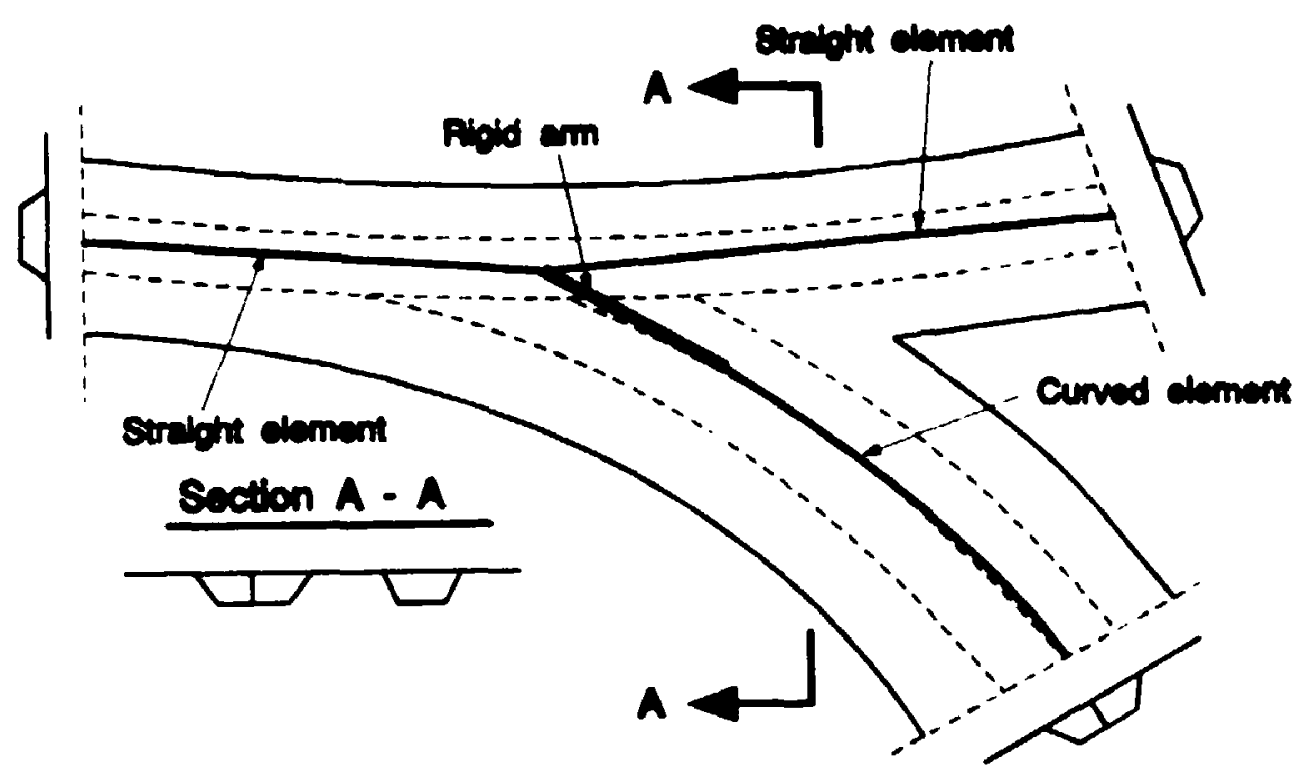

Fig. 4.12 Box girder assemblage.

enforce continuity of $u, v, w, \theta_{z}, u^{\prime}$ and $v^{\prime}$. This degree of continuity is identical to that required in conventional beam or frame assemblages. The other degrees of freedom, such as torsional warping $\theta_{2}^{\prime}$, distortion $\beta_{1}$, distortional warping $\beta_{1}^{\prime}$, shear $\operatorname{lag} \psi_{i}$ and shear lag warping $\psi_{i}^{\prime}$, are treated as local degrees of freedom, reflecting the local deformations of the individual cross sections. Those degrees of freedom for which continuity must be enforced are therefore transformed to a common global coordinate system. Figure $4.13(\mathrm{c})$ is the combination of Fig. $4.13(\mathrm{a})$ and $4.13(\mathrm{~b})$, which shows that between elements 1 and 2 full continuity is maintained while between element 3 and the preceding two elements only partia! continuity is enforced as in Fig. 4.13(b).

For the straight box beam element, it may be recalled that the element stiffness matrix in Eq. (3.5.11) and the equivalent nodal load vector in Eq. (3.5.13) are based on the local orthogonalized coordinate system $\{D\}$. Before we can perform global coordinate transformation, the foregoing stiffness matrix and load vector for each element have to be transformed into the element local coordinate system $\{D\}$. For the convenience of coordinate transformation, the element nodal degrees of freedom vector, $\{\bar{D}\}^{e}$ in Eq. (3.5.1), is reordered as shown below: 


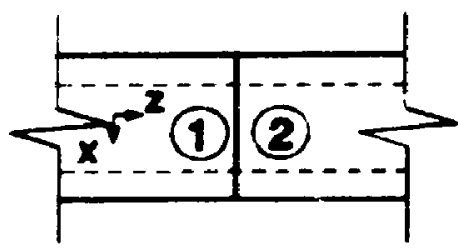

(a)

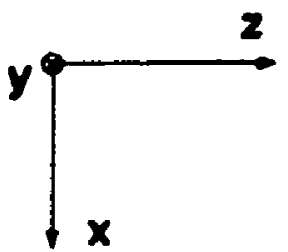

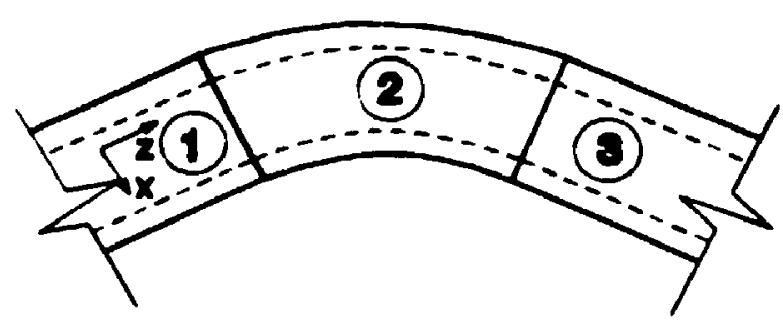

(b)

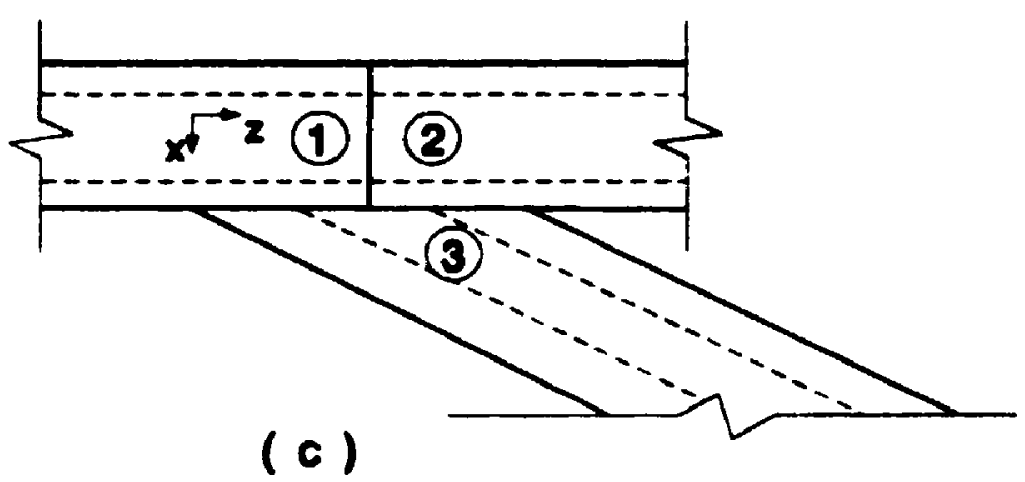

Fig. 4.13 Intersections of box girder bridge assemblages.

$$
\begin{aligned}
\{\bar{D}\}^{e}= & {\left[\begin{array}{llllllllll}
\bar{u}_{i} & \bar{u}_{1} & \bar{v}_{i} & \bar{\theta}_{z i} & \bar{\beta}_{1 i} & \ldots & \bar{\beta}_{n i} & \bar{\psi}_{11} & \ldots & \bar{\psi}_{m i} \\
& \bar{u}_{i}^{\prime} \bar{v}_{i}^{\prime} & \vec{\theta}_{x i} & \bar{\beta}_{1 i}^{\prime} & \ldots & \bar{\beta}_{n i}^{\prime} & \bar{\psi}_{1 i}^{\prime} & \ldots & \bar{\psi}_{m i}^{\prime} \\
& \bar{w}, \bar{u}_{,} \bar{v}_{j} & \bar{\theta}_{z}, & \bar{\beta}_{1}, & \ldots & \bar{\beta}_{n j} & \bar{\psi}_{1 j} & \ldots & \bar{\psi}_{m j} \\
& \bar{u}_{j}^{\prime} & \bar{v}_{j}^{\prime} & \bar{\theta}_{z}^{\prime}, & \bar{\beta}_{1}^{\prime} & \ldots & \bar{\beta}_{n j}^{\prime} & \bar{\psi}_{1 j}^{\prime} & \ldots & \bar{\psi}_{m j}^{\prime}
\end{array}\right]^{T} }
\end{aligned}
$$

The coordinate transformation between $\{D\}^{e}$ and $\{\bar{D}\}^{e}$ can be found from Eq. (3.3.12)

$$
\{D\}^{e}=\left[T_{p}\right]^{-1}\{\bar{D}\}^{e}
$$

where $\quad\left[T_{p}\right]=\left[\begin{array}{llll}{[T]} & & & \\ & {[T]} & & \\ & & {[T]} & \\ & & & {[T]}\end{array}\right]$

and the matrix $[T]$ is the same as defined in Eq. (3.3.11). Hence, the stiffness matrix and the equivalent nodal load vector corresponding to the element local coordinate system $\{D\}$ can be obtained from

$$
[S]^{e}=\left[T_{p}\right]^{-1}[\bar{S}]^{e}\left[T_{p}\right]
$$




$$
\{F\}^{e}=\left\{T_{p}\right\}^{-1}\{\bar{F}\}^{e}
$$

The global coordinate system is shown in Fig. 4.13. For bridge structures, generally the local $y$ axis in Fig. 4.13 coincides with the global $y^{\prime}$ axis and thus no transformation is needed about this axis. Accordingly, coordinate transformation between the element local coordinates and the structural global coordinates is given by

$$
\{D\}^{e}=\left[T_{g}\right]\left\{D_{g}\right\}^{e}
$$

with

$$
\left[T_{g}\right]=\left[\begin{array}{ll}
{[t]} & \\
& {[t]}
\end{array}\right]
$$

where the submatrix $[t]$ is as follows

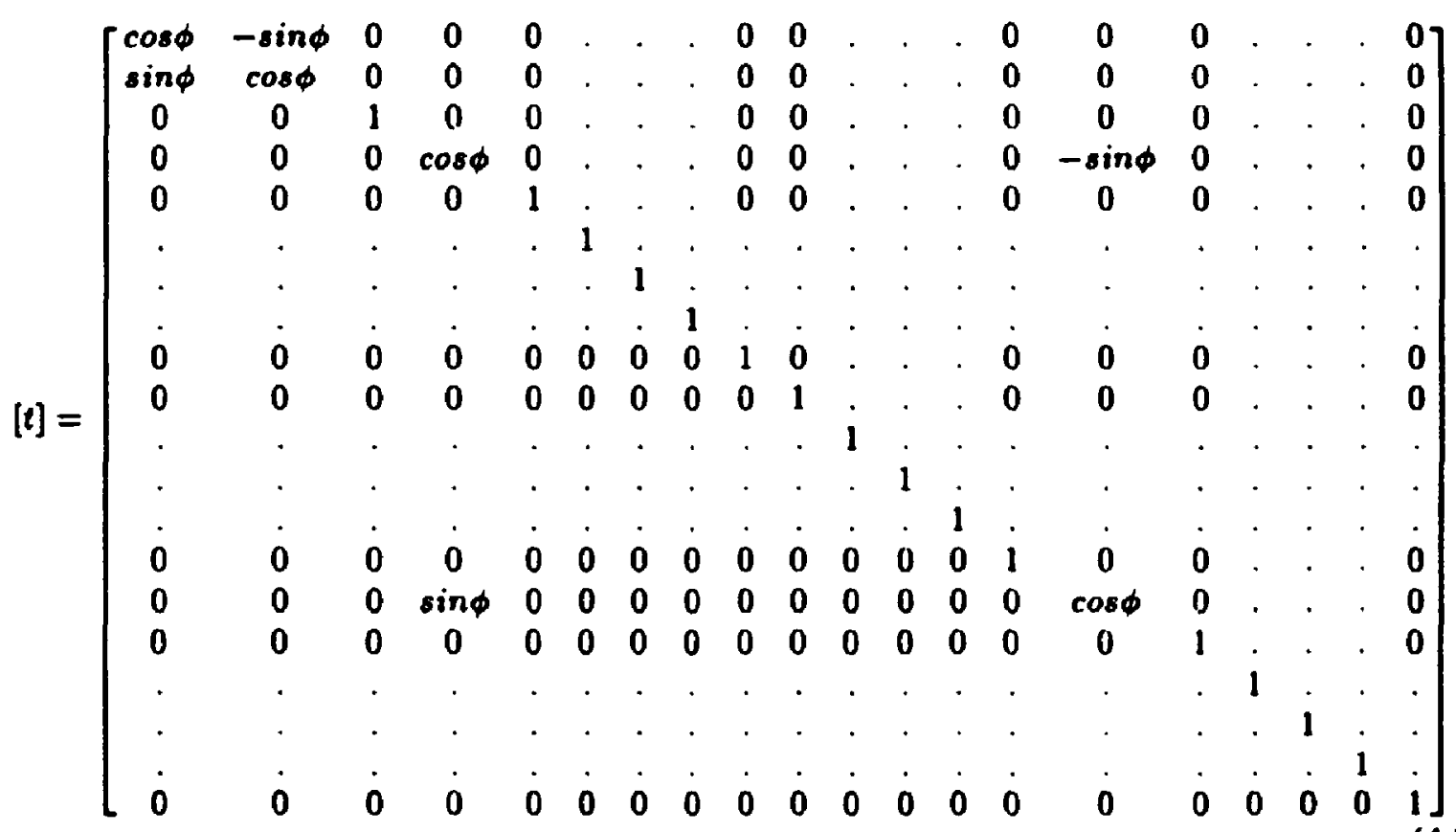

in which $\phi$ is the angle between the local $z$ axis and the global $Z$ axis. Note that the off diagonal elements of the dotted rows and columns are equal to zero. Matrix $[t]$ has dimensions of $2 \bar{m} \times 2 \bar{m}$. Consequently, the element stiffness matrix and the equivalent nodal load vector corresponding to the global coordinate system can be obtained from

$$
\begin{gathered}
{\left[S_{g 1}\right]^{e}=\left[T_{g}\right]^{T}[S]^{e}\left[T_{g}\right]} \\
\left\{F_{g 1}\right\}^{e}=\left\{T_{g}\right]^{T}\{F\}^{e}
\end{gathered}
$$


For curved box beam element, the nodal degrees of freedom after eliminating the internal degrees of freedom by static condensation is given by

$$
\begin{aligned}
& \left\{D_{c}\right\}^{e}=\left[\begin{array}{lllllllll}
u_{1} & v_{1} & w_{1} & \theta_{z 1} & \theta_{y 1} & \theta_{21} & \theta_{z 1}^{\prime} & \beta_{11} & \beta_{11}^{\prime}
\end{array}\right. \\
& \begin{array}{lllllllllll}
\ldots & \beta_{n 1} & \beta_{n 1}^{\prime} & \psi_{11} & \psi_{11}^{\prime} & \ldots & \psi_{m 1} & \psi_{m 1}^{\prime}
\end{array} \\
& \begin{array}{lllllllllll}
u_{3} & v_{3} & w_{3} & \theta_{x 3} & \theta_{y 3} & \theta_{23} & \theta_{23}^{\prime} & \beta_{13} & \beta_{13}^{\prime} & \ldots
\end{array} \\
& \left.\begin{array}{llllllll}
\beta_{n 3} & \beta_{n 3}^{\prime} & \psi_{13} & \psi_{13}^{\prime} & \ldots & \psi_{m 3} & \psi_{m 3}^{\prime}
\end{array}\right]^{T}
\end{aligned}
$$

The displacement continuity conditions between straight box element and curved element are listed as follows

$$
\begin{gathered}
u=u_{0}+y_{s} \theta_{z} \\
v=v_{0} \\
w=w_{0}+y_{c} v^{\prime}+\omega \theta_{2}+\omega_{d 1} \beta_{1}+\ldots+\omega_{d n} \beta_{n}+ \\
\omega_{v 1} \psi_{1}+\ldots+\omega_{v m} \psi_{m} \\
v^{\prime}=-\theta_{x} \\
u^{\prime}=\theta_{y}+y_{c} \theta_{z}^{\prime} \\
\theta_{z}=\theta_{2} \\
\beta_{1}=\beta_{1} \\
\beta_{n}=\beta_{n} \\
\psi_{1}^{\prime}=\psi_{1} \\
\psi_{m}=\psi_{m}
\end{gathered}
$$

Thus, the transformation between the nodal degrees of freedom can be expressed as

$$
\{D\}^{e}=\left[T_{s c}\right]\left\{D_{c}\right\}^{e}
$$

with

$$
\left[T_{s c}\right]=\left[\begin{array}{ll}
{\left[t_{s c}\right]} & \\
& {\left[t_{s c}\right]}
\end{array}\right]
$$


where the submatrix $\left[t_{a c}\right]$ is as follows

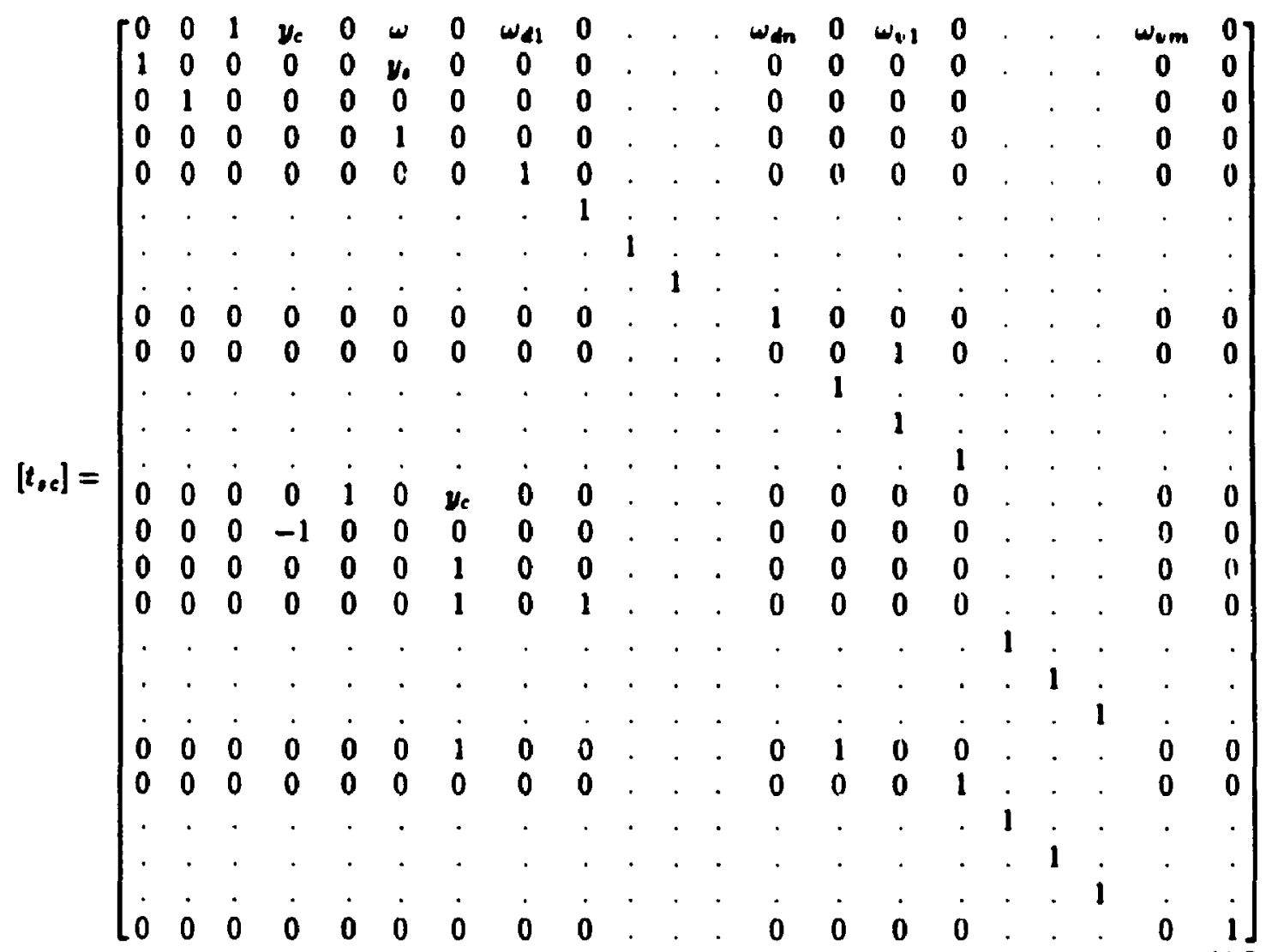

in which $y_{c}$ and $y_{s}$ are the coordinates of the centroid and the shear center of the cross section, respectively, and $\omega, \omega_{d 1}$ to $\omega_{d n}$ and $\omega_{v 1}$ to $\omega_{v m}$ are the torsional, distortional and shear lag warping functions at point $O$, respectively. The element stiffness matrix and the nodal load vector for the straight element corresponding to the global coordinate system can be obtained from

$$
\begin{gathered}
{\left[S_{g 2}\right]^{e}=\left[T_{s c}\right]^{T}\left[S_{g 1}\right]^{e}\left[T_{s c}\right]} \\
\left\{F_{g 2}\right\}^{e}=\left[T_{s c}\right]^{T}\left\{F_{g 1}\right\}^{e}
\end{gathered}
$$

In the intersection region, the bridge stiffness is very large compared to the other parts of the structure. Thus rigid arm is used to model this local area. The transformation matrix is similar to Eqs. (4.5.14) and (4.5.15) and is listed as following:

$$
\left[T_{r a}\right]=\left[\begin{array}{ll}
\left|t_{r b}\right| & \\
& {\left[t_{r a}\right]}
\end{array}\right]
$$


where the submatrix $\left[t_{\text {ra }}\right]$ is

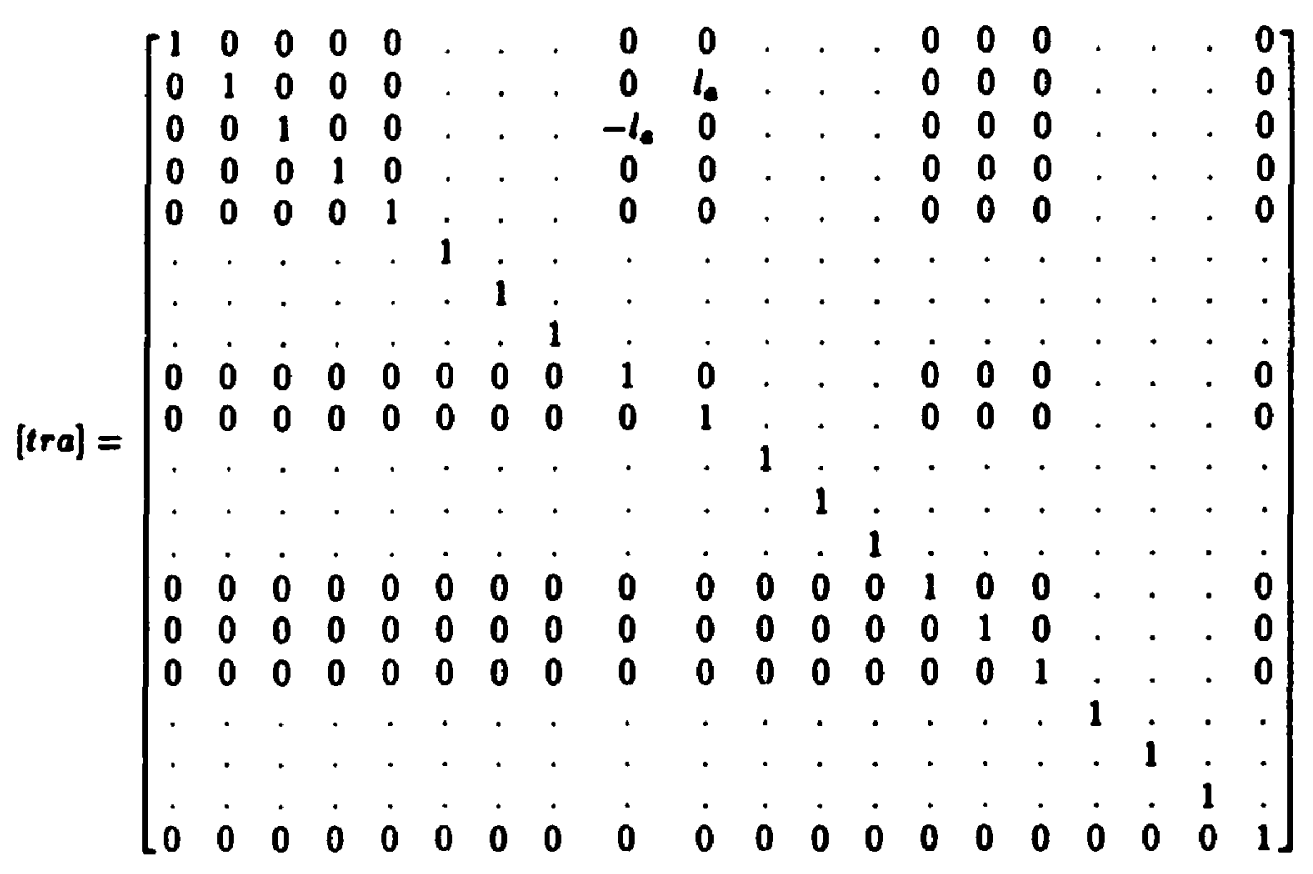

in which $l_{a}$ is the length of rigid arm of the element. The corresponding element stiffness matrix and nodal load vector can be found from

$$
\begin{gathered}
{\left[S_{g j^{e}}=\left[T_{a r}\right]^{T}\left[S_{g 2}\right]^{e}\left[T_{a r}\right]\right.} \\
\left\{F_{g}\right\}^{e}=\left[T_{a r}\right]^{T}\left\{F_{g 2}\right\}^{e}
\end{gathered}
$$

The global coordinate system coincides with the element coordinate system for the curved element, thus no coordinate transformation is needed for the element stiffness matrix and nodal load vector of the curved element. Once the necessary transformations have been achieved, the process of assembly of the structural stiffness matrix and load vectors proceeds in the usual manner. The accuracy that can be obtained with the proposed procedure will be demonstrated later in Chapter 6 . 


\section{PRESTRESSING, TEMPERATURE, CREEP AND SHRINKAGE EFFECTS}

\subsection{General}

Prestressing, thermal, creep and shrinkage effects are very important for concrete box girder bridges and therefore the analysis of box girders would be incomplete without taking these important effects properly into account. For the purpose of the present investigation, prestressing can be analyzed by developing a one dimensional prestressing bar element, and the prestressing force can be treated as equivalent nodal forces. Insofar as thermal stresses are concerned, different temperature distributions over the box girders would generate different stresses in a structure; therefore, first some available temperature distributions for bridges will be presented. Priestley's (1976) suggested temperature distribution will be used in conjunction with the thinwalled beam theory presented in Section (5.3) to study thermal stresses.

Creep and shrinkage are time-dependent phenomena. Generally, these do not affect the strength of a structure, but for serviceability limit states they can not be ignored. In Section (5.4), creep and shrinkage are further discussed and the procedures for time-dependent stress analysis are presented. The equivalent nodal forces due to creep and shrinkage are derived based on the finite element formulation given in the previous chapter. It should be emphasized that the proposed formulation enables one to determine the influence of prestressing and thermal effects on the distortion and warping of the cross section. 


\subsection{Prestressing Bar Element}

In practical engineering, most concrete box girder bridges are prestressed, and therefore prestressing is one of the important factors which should be included in the analysis. To simplify the analysis, the method presented in this section is based on the following assumptions:

(a) The weakening of the cross section by the holes provided for the prestressing bar may be neglected.

(b) No bond slip is allowed between the prestressing bars and the concrete

Thus, the prestressing force can be simply considered as a particular loading case and the equivalent element nodal load vector can be obtained by finite element procedures. The stiffness of the prestressing bar can be systematically included in the analysis by developing a special prestressing bar finite element.

Figure 5.1 shows a general prestressing bar element embedded in the wall of a box beam element. The bar is assumed to lie on the middle surface of the wall and it contributes only to the in plane stiffness of the element. Local coordinates $y$ and z define the geometry of the prestressing bar and may be expressed as follows:

$$
y=y_{1} M_{1}(\bar{\zeta})+y_{2} M_{2}(\bar{\zeta})+y_{3} M_{3}(\bar{\zeta})=\sum_{i=1}^{3} y_{i} M_{i}(\bar{\zeta})
$$

where the shape functions $M_{i}(\bar{\zeta})$ are defined as

$$
\begin{aligned}
& M_{1}(\bar{\zeta})=\frac{1}{2}\left(\bar{\zeta}^{2}-\bar{\zeta}\right) \\
& M_{2}(\bar{\zeta})=\left(1-\bar{\zeta}^{2}\right) \\
& M_{3}(\bar{\zeta})=\frac{1}{2}\left(\bar{\zeta}^{2}+\bar{\zeta}\right)
\end{aligned}
$$

In the above $\bar{\zeta}=2 z / l$ is a non-dimensional parameter (natural coordinate) varying from -1 to +1 between the end points of the bar and $l$ is the length of the bar as indicated in Fig. 5.1; $y_{1}, y_{2}$ and $y_{3}$ are the coordinates of the nodal points which, in the computer program developed, are input as data, or generated from simliar data for other elements. 


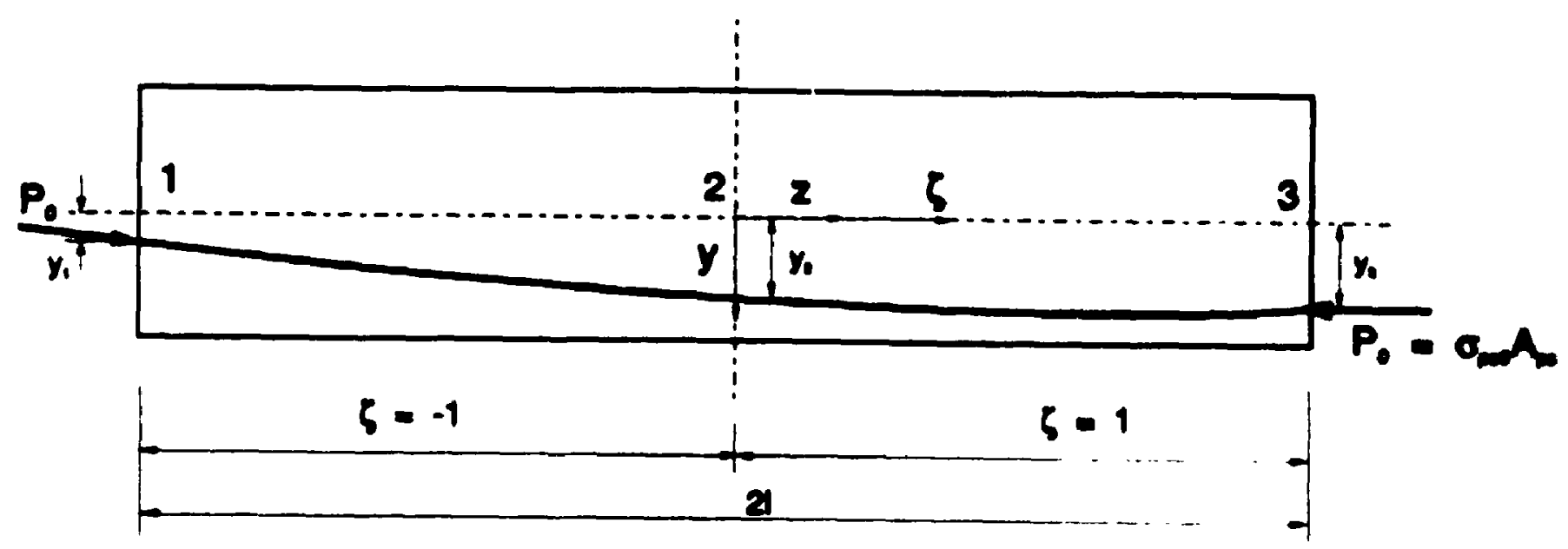

Fig. 5.1 Prestreased bar element.

Based on the assumption (b) and from Eq. (4.4.7), the strain vector at any point in the bar is given by

$$
\{\epsilon\}=[B]\{D\}^{e}+\left\{\epsilon_{0}\right\}
$$

where $\left\{\epsilon_{0}\right\}$ is the initial strain vector in the prestressing bar. Hence, the longitudinal normal strain $\epsilon_{p}$ at any point at a distance $s$ from the end of the bar can be calculated as

$$
\epsilon_{p s}=[t]\{\epsilon\}=[t][B]\{D\}^{e}+\epsilon_{p, 0}
$$

where

$$
\epsilon_{p s 0}=[t]\left\{\epsilon_{0}\right\}
$$

and $[t]$ is the strain transfor.nation matrix given by:

$$
[t]=\left[\begin{array}{lll}
\cos ^{2} \alpha & \sin ^{2} \alpha-\sin \alpha \cos \alpha
\end{array}\right]
$$

in which $\alpha$ is the angle between the $z$-axis and the tangent to the prestressing cable. The corresponding longitudinal normal stress $\sigma_{p}$ is given by

$$
\sigma_{p p}=E_{p e}\left(\epsilon_{p p}-\epsilon_{p p 0}\right)+\sigma_{p p 0}=E_{p p}[t][B]\{D\}^{e}+\sigma_{p p 0}
$$

where $E_{p s}$ is the Young's modulus of the prestressing bar, and $\sigma_{p, 0}$ is the initial stress in the prestressing bar. 
The prest ressing bas element stiffness matrix, $\left[S_{p,}\right]^{e}$, is given by:

$$
\begin{aligned}
{\left[S_{p p}\right]^{e} } & =\int_{p}[B]^{T}[t]^{T} E_{p,} A_{p,}[t][B] d s=\int_{-1 / 2}^{1 / 2}[B]^{T}[t]^{T} E_{p,} A_{p,}[t][B] \sqrt{1+y^{\prime 2}} d z \\
& =\int_{-1}^{1}[B]^{T}[t]^{T} E_{p,} A_{p o}[t][B] \sqrt{1+y^{\prime 2}}(l / 2) d \zeta
\end{aligned}
$$

and

$$
d s=\sqrt{1+y^{\prime 2}} d z
$$

where $A_{p a}$ is the area of the prestressing bar. The equivalent nodal load vectors due to initial strain $t_{p, 0}$ and initial stress $\sigma_{p, 0}$ are given, respectively, by:

$$
\begin{aligned}
& \left\{F_{p s}\right\}_{e p o 0}^{e}=\int_{0}[B]^{T}[t]^{T} A_{p,} E_{p, e_{p, 0}} d s=\int_{-1 / 2}^{l / 2}[B]^{T}[t]^{T} A_{p,} E_{p s e_{p, 0}} \sqrt{1+y^{\prime 2}} d z \\
& =\int_{-1}^{1}[B]^{T}[t]^{T} A_{p,} E_{p, C} C_{p o 0} \sqrt{1+y^{\prime 2}}(l / 2) d \zeta
\end{aligned}
$$

and

$$
\begin{aligned}
\left\{F_{p s}\right\}_{a_{p p 0}}^{e} & =-\int_{0}[B]^{T}[t]^{T} A_{p, \sigma_{p, 0} a^{\prime} s}=-\int_{-1 / 2}^{1 / 2}[B]^{T}[t]^{T} A_{p, \sigma_{p, 0}} \sqrt{1+y^{\prime 2}} d z \\
& =-\int_{-1}^{1}[B]^{T}[t]^{T} A_{p,} \sigma_{p, 0} \sqrt{1+y^{\prime 2}}(1 / 2) d \zeta
\end{aligned}
$$

In Eq. (5.2.9b), a constant prestressing force is assumed through the length of the prestressing bar. Since the prestressing force may vary along the tendon and in order to account for this variation, one should divide the bar into several discrete elements, each with a constant prestressing force. The integration in Eqs. (5.2.8), (5.2.9a) and (5.2.9b) is performed numerically by the Gauss quadrature rule.

\subsection{Effects of Temperature}

In bridge structures exposed to large temperature variations, substantial thermal stresses can develop. Bridges are usually provided with expansion joints and bearings which allow certain movements in the superstructure. Furthermore, a linear temperature distribution over the cross section of a statically determinate structure 
does not produce stresses. On the other hand, when the temperature distribution is non-linear, the structure will be subjected to thernial stresses because the fibers, being attached to each other, cannot expand freely. In a statical!y indeterminate structure, a linear or non-linear temperature distribution produces statically indeterminate reactions and internal forces. In finite element analysis, temperature can be treated as a special loading cases and is analogous to shrinkage analysis.

\subsubsection{Temperature Distribution in Box Girder Bridges}

The temperature distribution over a bridge can be obtained from experimental data or from heat transfer analysis. For practical applications, it is sufficient to assume that the temperature at any instant is constant over the bridge length, but variable over the cross section. The temperature distribution over a bridge cross section varies with time and depends upon the following factors (Ghali and Favre 1986):

1. Geometry of the cross section;

2. Thermal conductivity, specific heat and density of the material;

3. Nature and colour of the exposed surfaces, expressed in terms of solar radiation absorptivity, emissivity and convection coefficients;

4. Orientation of the bridge axis, latitude and altitude of the bridge location;

5. Time of the day and the season;

6. Diurnal variations of ambient air temperature and wind speed;

7. Degree of cloudiness and turbidity of the atmosphere.

Elbadry and Ghalj (1983) studied temperature distribution for some typical bridge cross sections as shown in Fig. 5.2. They concluded that concrete bridges of the same depth but with different cross sectional shapes have almost the same temperature distribution as shown in Fig. 5.3, but the temperature distribution and the resulting stresses vary considerably with the cross section depth. With greuter depth, higher temperature stresses occur as shown in Fig. 5.4. 


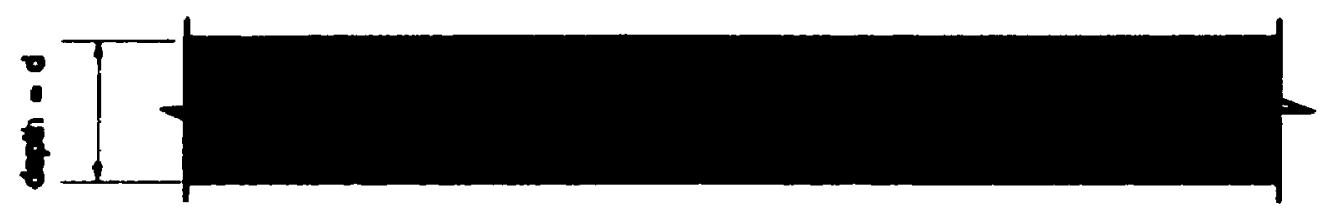

(a) Solid ainb

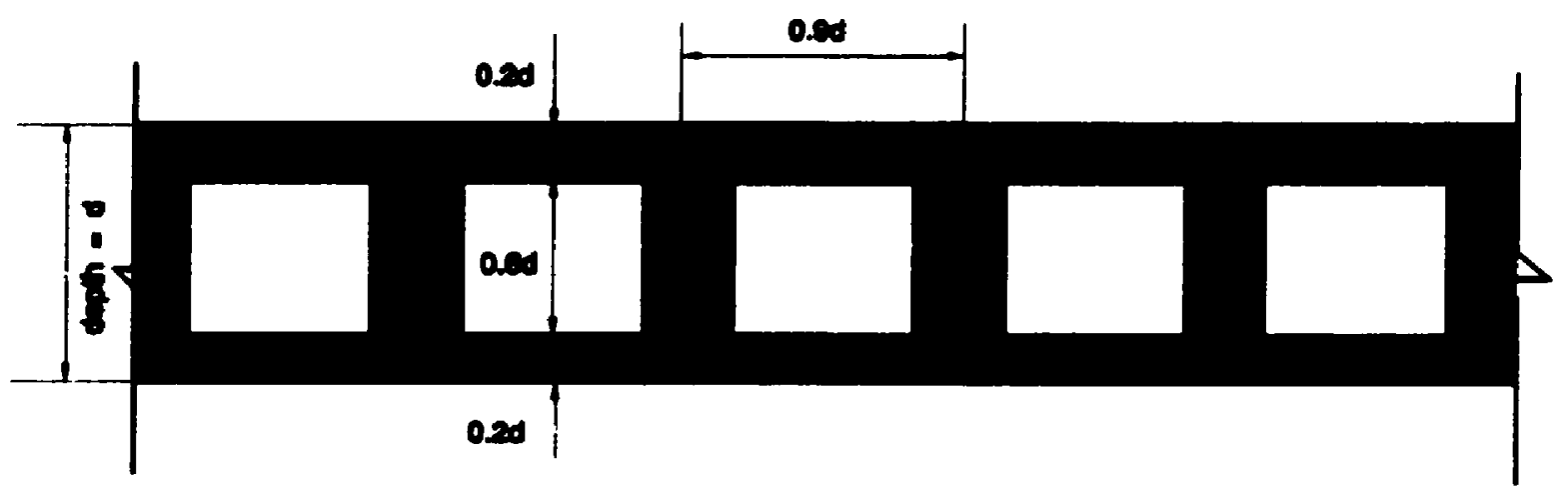

(b) Collutar slab

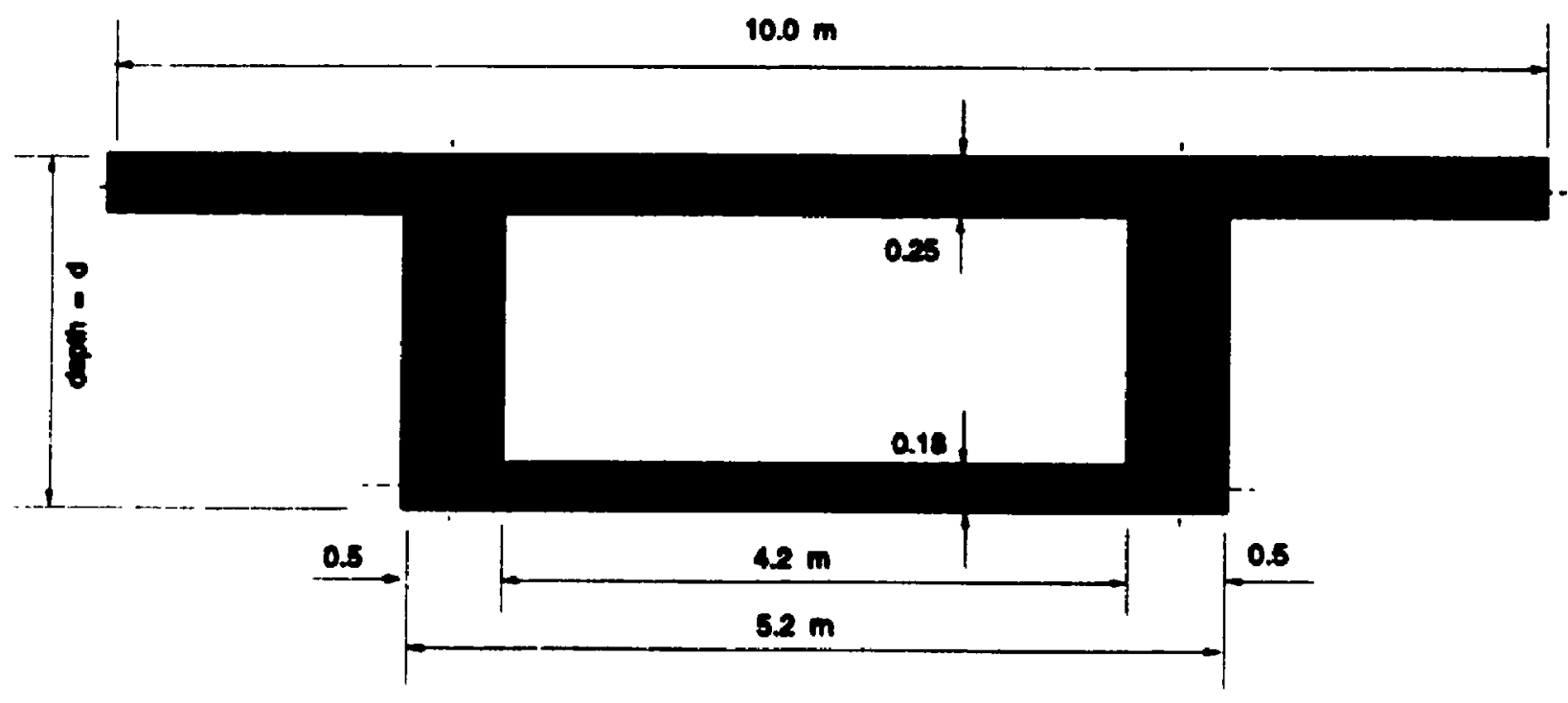

(c) Box girder

Fig. 5.2 Bridge cross sections analysed by Elbadry and Ghali. 


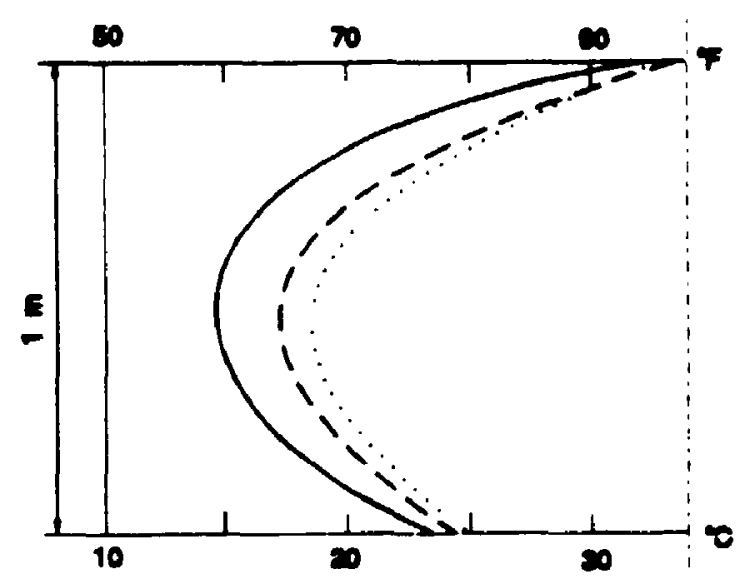

Temperaure

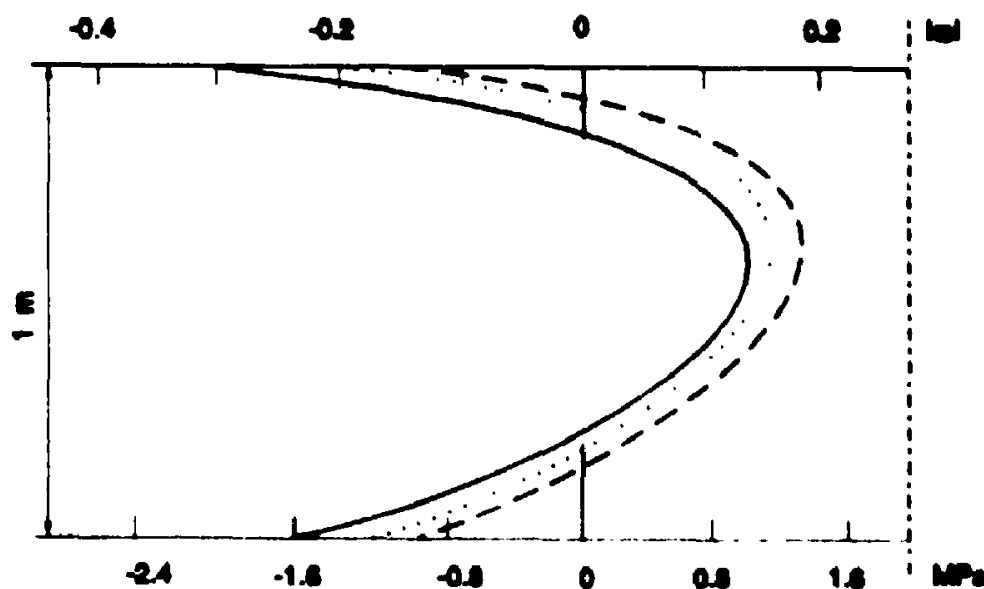

Salf - cquilubrating strece

Fig. 5.3 Temperature and stress distributions.

In this thesis, Priestley's (1976) temperature distribution, Fig. 5.5, is used for the thermal stress analysis of box girders. The distribution is a combination of straight lines and a fifth-degree parabola and was obtained from finite difference analyses. This temperature distribution respresents the conditions in the early afternoon of a hot summer day. A temperature distribution of the same form, but opposite sign, is often suggested for the conditions in winter during the night or early in the morning. It should be mentioned that determination of actual thermal profiles is not within the scope of the present work. The principal object of this chapter is to demonstrate how thin-walled beam theory can be utilized in conjunction with available thermal and creep data to better analyze box girder bridges.

\subsubsection{Nodal Forces and Thermal Stresses}

In finite element analysis, the temperature can be considered as a special loading case. The temperature expansion is assumed restrained first and the stresses produced by this artificially restrained condition are used to determine the nodal forces in the restrained structure. Then, the structure can be released by applying those nodal forces in the opposite directions. The released structure is then analyzed for the latter nodal forces. The procedure may be better described through a specific example.

Consider a multicell box girder having the cross section shown in Fig. 4.7, and 

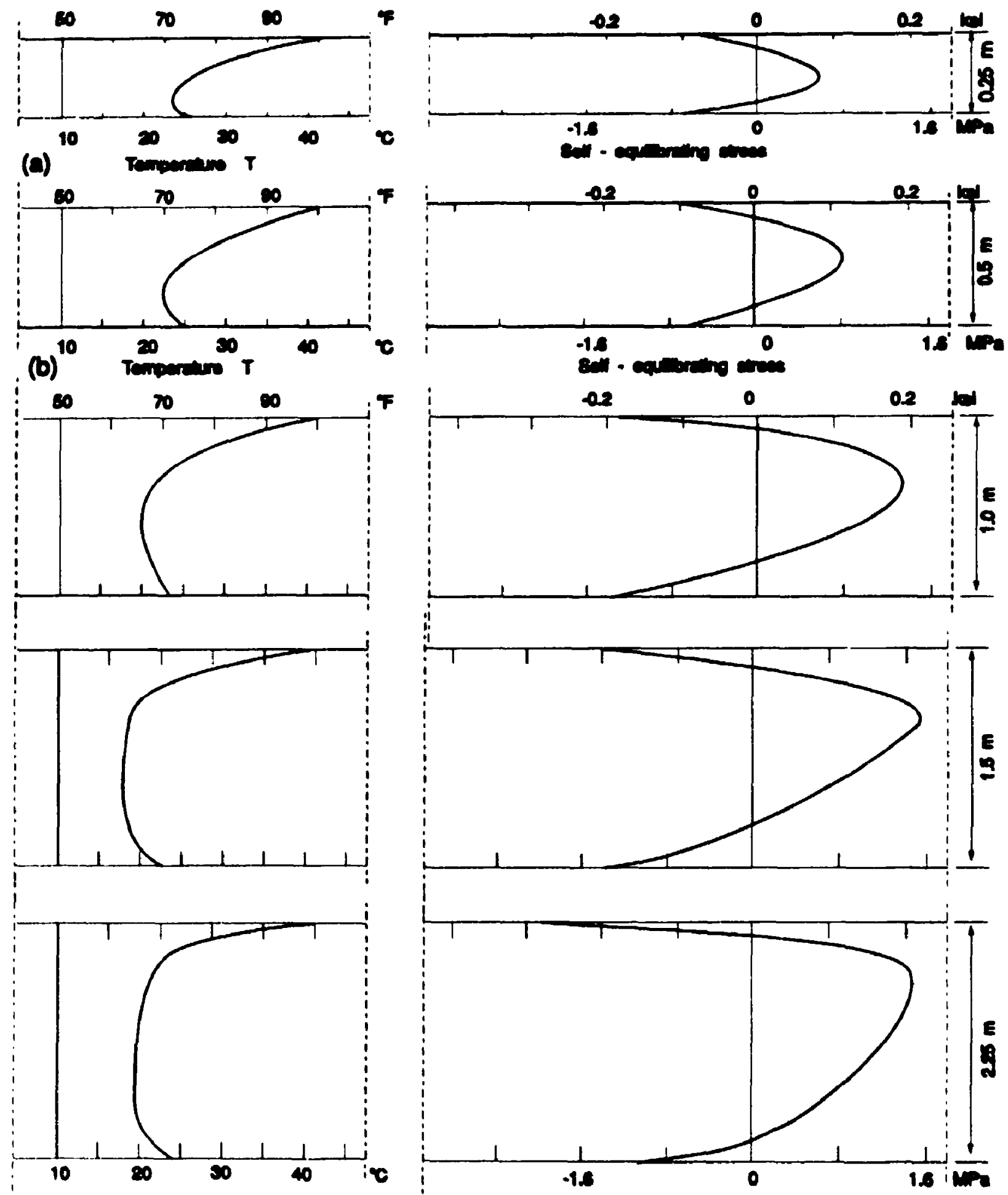

(c) Torpeners $T$

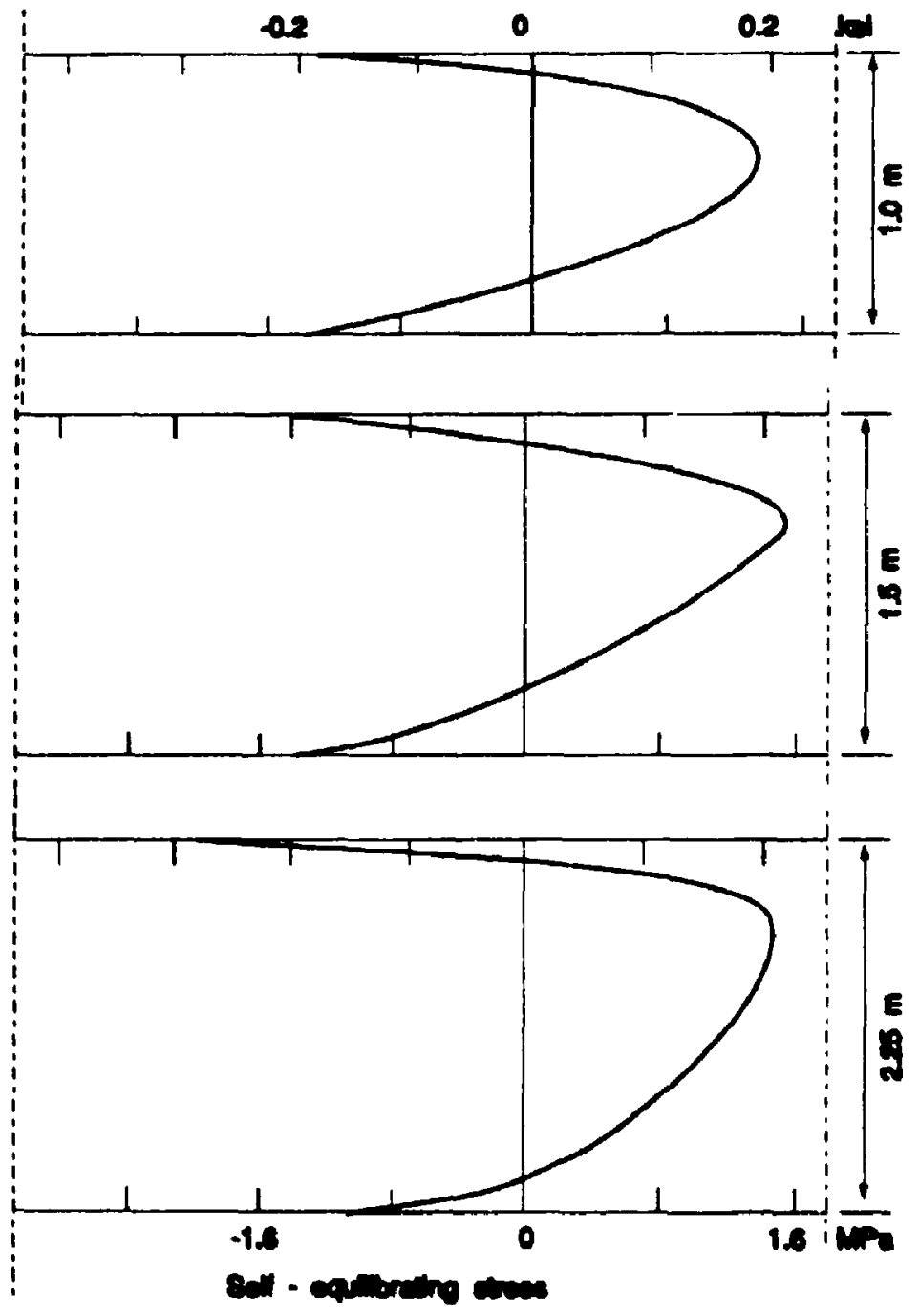

Fig. 3.4 Temperature and stress distributions. 


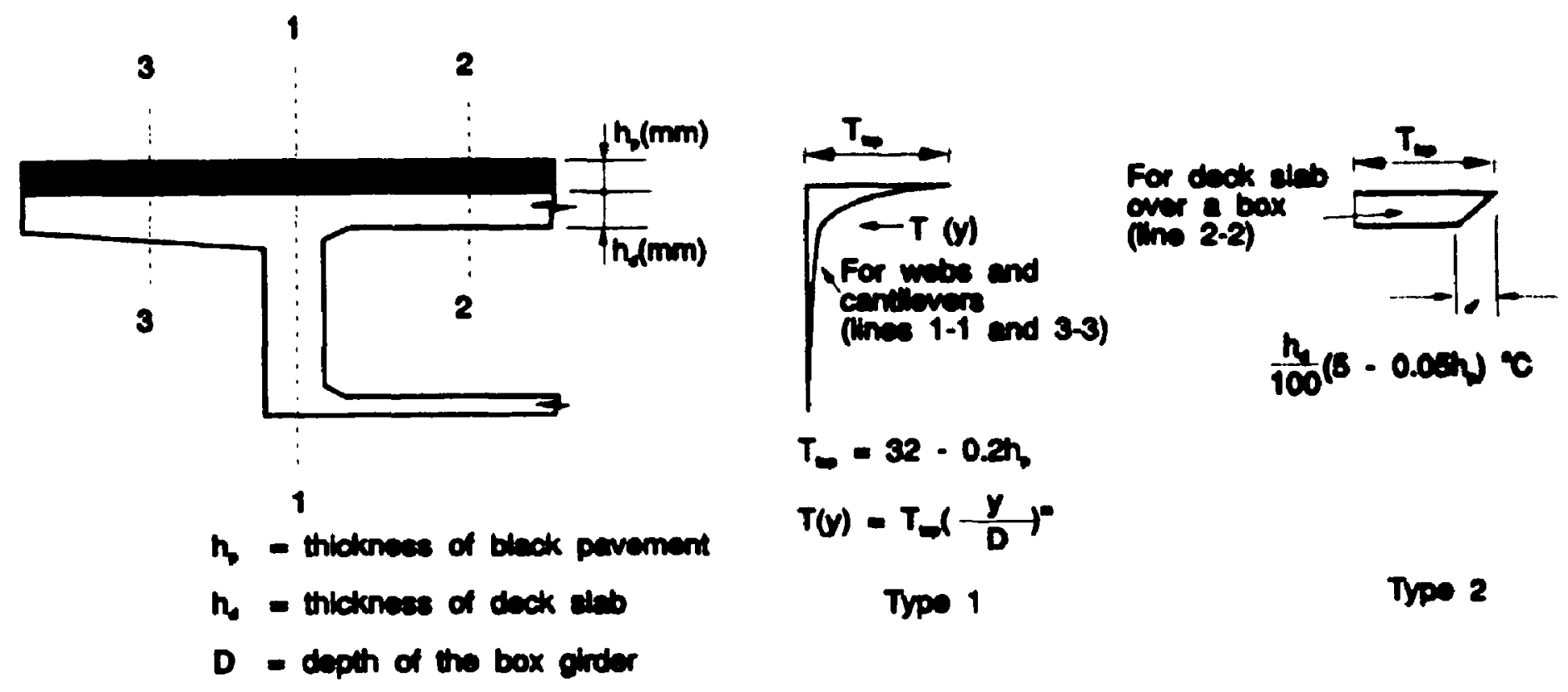

Fig. 5.5 Temperature distribution from Priestly.

let the distribution of temperature be given by function $T(x, y)$. If the temperature expansion is free, then the strains due to temperature change $T(x, y)$ will be given by

$$
\left\{\epsilon_{\ell}\right\}=\left\{\begin{array}{c}
\alpha_{2} T(x, y) \\
\alpha_{s} T(x, y) \\
0
\end{array}\right\}
$$

where $\alpha_{z}$ and $\alpha_{s}$ are the coefficients of thermal expansion in the $z$ and $s$ directions, respectively.

If those strains are artificially prevented, the stresses in the restrained condition will be

$$
\left\{\bar{\sigma}_{t}\right\}=-[E]\left\{\epsilon_{t}\right\}=\left\{\begin{array}{c}
\bar{\sigma}_{z t} \\
\bar{\sigma}_{s t} \\
0
\end{array}\right\}
$$

where

$$
\begin{aligned}
& \bar{\sigma}_{z t}=\frac{\alpha_{z}+\nu_{z} \alpha_{s}}{1-\nu_{z} \nu_{s}} E_{z} T \\
& \bar{\sigma}_{s t}=\frac{\nu_{s} \alpha_{z}+\alpha_{s}}{1-\nu_{z} \nu_{s}} E_{s} T
\end{aligned}
$$

The element equivalent nodal load vector due to stresses $\left\{\bar{\sigma}_{l}\right\}$ is given by

$$
\left\{F_{t}\right\}^{e}=\int_{V}[B]^{T}\left\{\bar{\sigma}_{t}\right\} d V=\int_{-1}^{+1} \int_{0}^{+1} \int_{-1}^{+1}[B]^{T}\left\{\bar{\sigma}_{t}\right\}|J| d \xi d \eta d \zeta
$$

Substituting the $[B]$ matrix from Eq. (4.4.) into the above equation, the vector 
$\left\{F_{1}\right\}^{e}$ becomes

$$
\begin{aligned}
\left\{F_{t}\right\}^{e}= & \int_{-1}^{+1} \int_{0}^{+1} \int_{-1}^{+1}\left(\bar{\sigma}_{21}\left[N^{\prime}\right]^{T}\left\{\Phi_{1}\right\}+\bar{\sigma}_{21}\left[N^{\prime \prime}\right]^{T}\left(\left\{\Phi_{2}\right\}-n\{\equiv\}\right)\right. \\
& \left.-\bar{\sigma}_{a t} n[N]^{T}\{\bar{\Xi}]\right)|J| d \xi d \eta d \zeta
\end{aligned}
$$

Once the element equivalent nodal load vector is found, customary finite element procedures as described in Sect. (3.2) can be followed and the stresses $\left\{\Delta \sigma_{1}\right\}$ due to the equivalent loads can be found. The actual stresses due to temperature are the summation of $\left\{\bar{\sigma}_{1}\right\}$ and $\left\{\Delta \sigma_{t}\right\}$ :

$$
\left\{\sigma_{t}\right\}=\left\{\tilde{\sigma}_{t}\right\}+\left\{\Delta \sigma_{t}\right\}
$$

In the proposed formulation, both the longitudinai and the transverse normal and shear stresses due to temperature can be calculated directly unlike other finite element beam models for box girders in which the transverse normal stresses can be determined only from a separate transverse analysis of the box. It should also be remarked that the $[B]$ matrix in Eq. (5.3.5) will produce equivalent nodal forces in the form of bimoments if the thermal distribution is unsymmetric with respect to the cross section.

\subsection{Time-Dependent Effects}

Due to creep and shrinkage, the stresses in the cross section of a statically determinate structure vary with time. In addition, when the structure is statically indeterminate, the internal forces and reactions are also time-dependent. For the analysis of time-dependent stresses and deformations, the time functions for strain or stress in concrete must be known. Based on the recommendations of ACI Committee 209 (1982), some equations for the time-dependent variation of the modulus of elasticity, creep and shrinkage of concrete are available for practical applications. The important parameters that affect these functions are included in the equations, but it is beyond the scope of this thesis to examine how these parameters vary. The interested reader may refer to the report of Committee 209 (1982). It should 
be mentioned that the analytical procedures presented here are independent of the choice of time function.

\subsubsection{Creep}

For concrete under sustained stress, the strain will increase with time due to creep. On the other hand, the stress will decrease under constant sustained strain, a phenomenon referred to as relaxation. Creep and relaxation are, therefore, two descriptions of the same phenomenon. The total strain is equal to the instantaneous strain plus the creep strain at time $t$, shown in Fig. 5.6, which can be expressed as follows

$$
\epsilon_{c}(t)=\frac{\sigma_{c}\left(t_{0}\right)}{E_{c}\left(t_{0}\right)}\left[1+\varphi\left(t, t_{0}\right)\right]
$$

where $\sigma_{c}\left(t_{0}\right)$ is the sustained stress at age $t_{0} ; E_{c}\left(t_{0}\right)$ is the modulus of elasticity of concrete at age $t_{0}$ and $\varphi\left(t, t_{0}\right)$ is the creep coefficient function, correponding to concrete age $t_{0}$ at time of loading and the age $t$ at which the strain is being calculated. The creep coefficient may vary depending on the quality of concrete, the ambient temperature and humidity, as well as the dimensions of the element being considered. Kabir (1976) has summarized the important parameters which affect the creep coefficient and has reviewed different creep coefficient functions available for analysis. In this thesis, Committee 209 (1982) equations will be used to develop an analytical model for box girder bridge creep analysis

For standard conditions defined by Committee 209, the creep coefficeient function $\varphi\left(t, t_{0}\right)$ is given by

$$
\varphi\left(t, t_{0}\right)=\frac{\left(t-t_{0}\right)^{0.6}}{10+\left(t-t_{0}\right)^{0.6}}
$$

where

$$
\varphi_{u}=\varphi\left(t_{\infty}, t_{0}\right)
$$

is the ultimate creep, (say 10,000 days) and for the age at loading, $t_{0}$. Coefficient p. can be estimated by

$$
\varphi_{u}=2.35 \gamma_{c}
$$

in which $\gamma_{c}$ is a correction factor. For a relative humidity of $40 \%$, an average rnember thickness of $0.15 \mathrm{~m}$, a volume-to-surface ratio of $0.0375 \mathrm{~m}$, and a temperature of $21^{\circ} \mathrm{C}$, 


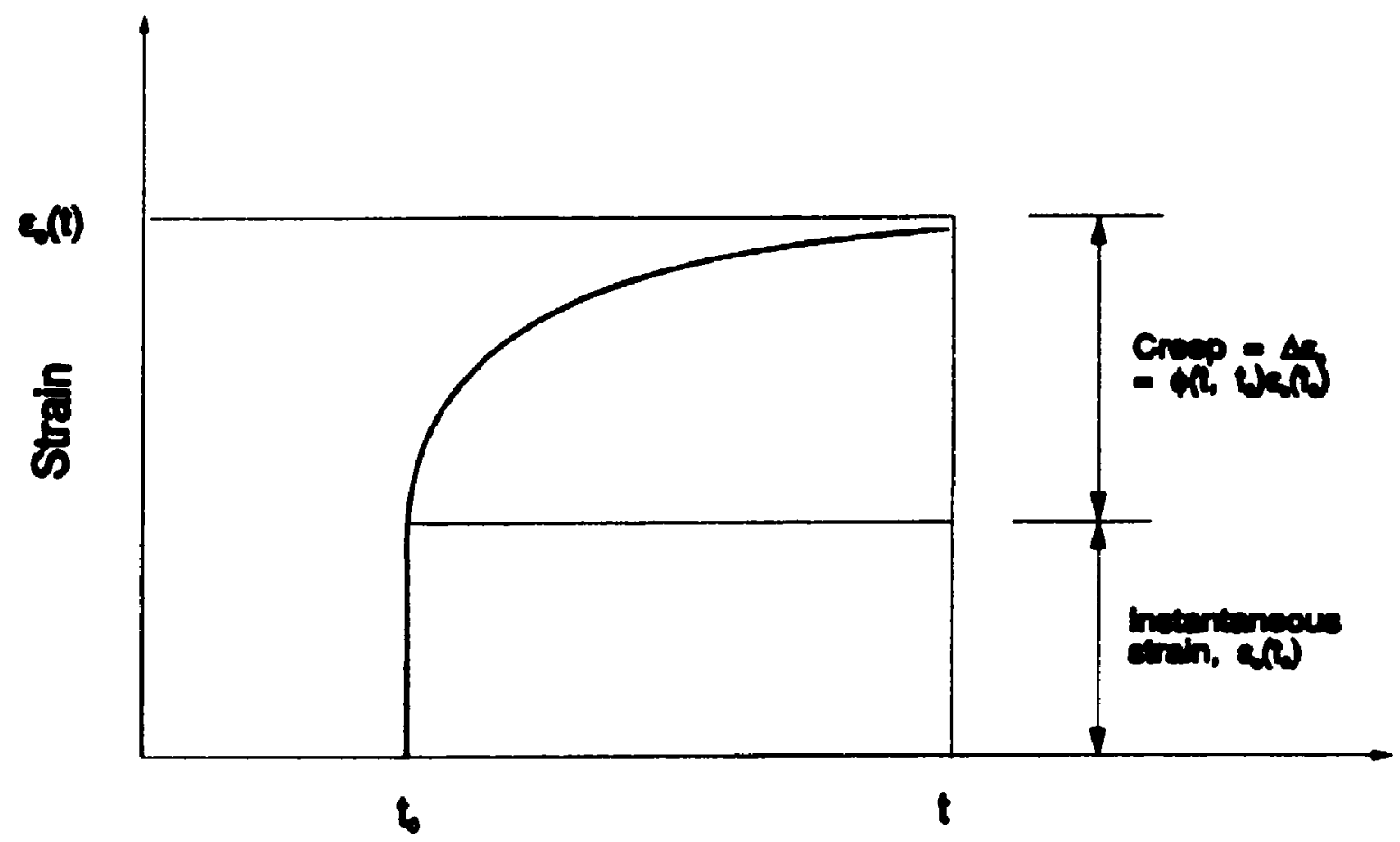

Time

Fig. 5.6 Creep of concrete under the effect of sustained stress.

$\gamma_{r}$ can be calculated as follows

For moist-cured concrete,

$$
\gamma_{c}=1.25 t_{0}^{-0.118}
$$

For $1-3$ days steam-cured concrete,

$$
\gamma_{c}=1.13 t_{0}^{-0.094}
$$

The two equations give $\gamma_{c} \simeq 1.0$ when $t_{0}=7$ and 3 days, respectively.

The modulus of elasticity of concrete is given by

$$
\frac{E_{c}\left(t_{0}\right)}{E_{c}(28)}=\left(\frac{t_{0}}{\alpha+\beta t_{0}}\right)^{1 / 2}
$$

where $a$ and $\beta$ are constants, depending on the type of cement and curing used. For cement Type $\mathrm{I}, \alpha=4$ days and $\beta=0.85$. 


\subsubsection{Shrinkage}

Shrinkage is defined by the volume change due to drying concrete. Stresses will be produced when the change of volume due to shrinkage is restrained. In box girder bridge structures, the restraint may be caused by the reinforcing or prest ressing steel, the supports or the difference in volume change in different parts of the structures. Creep and shrinkage usually occur simultaneously in concrete, and the stresses caused by shrinkage are generally reduced by the effect of creep. Thus, the effect of these two phenomena must be jointly considered in the analysis.

Based on the recommendations of Committee 209, for moist-cured concrete under standard conditions, the free shrinkage $\epsilon_{c s}$ which occurs between $t_{0}=7$ days and any time $t$ later is as follows

$$
\epsilon_{c s}\left(t, t_{0}\right)=\frac{t-t_{0}}{35+\left(t-t_{0}\right)}\left(\epsilon_{c s}\right)_{u} \quad \text { with } t_{0}=7
$$

For steam-cured concrete, when $t_{0}=1$ to 3 days, the free shrinkage $\epsilon_{c,}$ at time $t$ is given by

$$
\epsilon_{c s}\left(t, t_{0}=1 \text { to } 3\right)=\frac{t-(1 \text { to } 3)}{55+(t-1 \text { to } 3)}\left(\epsilon_{c o}\right)
$$

where $\left(\epsilon_{c s}\right)_{y}$ is the ultimate free shrinkage corresponding to time infinity, $t_{\infty}$ (say 10,000 days), and which is given by

$$
\left(\epsilon_{c s}\right)_{u}=-780 \times 10^{-6} \gamma_{c s}
$$

in which $\gamma_{c s}$ is the correction factor similar to $\gamma_{c}$ mentioned above. The factor $\gamma_{c s}$ is equal to one for initial moist curing of 7 days, a relative humidity of $40 \%$, an average thickness $0.15 \mathrm{~m}$, and a volume-to-surface ratio of $0.0375 \mathrm{~m}$.

\subsubsection{Time-Dependent Analysis}

The total strain $\epsilon_{c}(t)$ at time $t$ due to applied loads and shrinkage can be detcrmined from

$$
\epsilon_{c}(t)=\sigma_{c}\left(t_{0}\right) \frac{1+\varphi\left(t, t_{0}\right)}{E_{c}\left(t_{0}\right)}+\int_{\sigma_{e}\left(t_{0}\right)}^{\sigma_{e}(t)} \frac{1+\varphi(t, \tau)}{E_{c}(\tau)} d \sigma_{c}(\tau)+\epsilon_{c s}\left(t, t_{0}\right)
$$


where $\tau$ is an intermediate age between $t_{0}$ and $t ; d \sigma(\tau)$ is an elemental stress (increment or decrement) applied at age $T$. The integral in Eq. (5.4.11) can be greatly simplified by introducing an aging coefficient $\chi=\chi\left(t, t_{0}\right)$ as follows

$$
\epsilon_{c}(t)=\sigma_{c}\left(t_{0}\right) \frac{1+\varphi\left(t, t_{0}\right)}{E_{c}\left(t_{0}\right)}+\frac{\Delta \sigma_{c}(t)}{\vec{E}_{c}\left(t, t_{0}\right)}+\epsilon_{c o}\left(t, t_{0}\right)
$$

where $\Delta \sigma_{c}(t)$ is the total stress change during the period $t_{0}$ to $t$ shown in Fig. 5.7; $E_{c}\left(t, t_{0}\right)$ is the age-adjusted elasticity modulus which is given by

$$
\bar{E}_{c}\left(t, t_{0}\right)=\frac{E_{c}\left(t_{0}\right)}{1+\chi \varphi\left(t, t_{0}\right)}
$$

and the magnitude of $\chi$ is between 0 and 1 . The coefficient $\chi$ can be calculated from the relaxation function of concrete through a step by step numerical integration process (Ghali and Favre 1986). The stress at time $t$ due to sustained strain $\epsilon_{c}$ can be expressed as

$$
\sigma_{c}(t)=\epsilon_{c} r\left(t, t_{0}\right)
$$

where $r\left(t, t_{0}\right)$ is the relaxation function which can be defined as the stress at time $t$ due to a unit strain introduced at age $t_{0}$ and sustained constant during the period $t$ to $t_{0}$.

In order to find the relationship between $\chi$ and $r\left(t, t_{0}\right)$, we first substitute Eq. (5.4.13) into Eq. (5.4.12), then the strain at time $t$ can be written as

$$
\epsilon_{c}=\sigma_{c}\left(t_{0}\right) \frac{1+\varphi\left(t, t_{0}\right)}{E_{c}\left(t_{0}\right)}+\Delta \sigma_{c}(t) \frac{1+\chi \varphi\left(t, t_{0}\right)}{E_{c}\left(t_{0}\right)}
$$

where $\sigma_{c}\left(t_{0}\right)=\epsilon_{c} E_{c}\left(t_{0}\right)$, and $\Delta \sigma_{c}(t)=\sigma_{c}(t)-\sigma_{c}\left(t_{0}\right)$, as shown in Fig. 5.8. By substituting Eq. (5.4.14) into Eq. (5.4.15), $\epsilon_{c}$ may be expressed as

$$
\epsilon_{c}=\epsilon_{c}\left[1+\varphi\left(t, t_{0}\right)\right]+\epsilon_{c}\left[r\left(t, t_{0}\right)-E_{c}\left(t_{0}\right)\right] \frac{1+\chi \varphi\left(t, t_{0}\right)}{E_{c}\left(t_{0}\right)}
$$

From the definition of the relaxation function, the strain $\epsilon_{c}$ is constant and can therefore be removed from Eq. (5.4.16). After some algebraic manipulations of Eq. (5.4.16), the aging coefficient can be expressed as

$$
\chi\left(t, t_{0}\right)=\frac{1}{1-r\left(t, t_{0}\right) / E_{c}\left(t_{0}\right)}-\frac{1}{\varphi\left(t, t_{0}\right)}
$$

In Eq. (5.4.17) the only unknown is the relaxation function $r\left(t, t_{0}\right)$ which can be calculated through a step by step numerical integration process. 


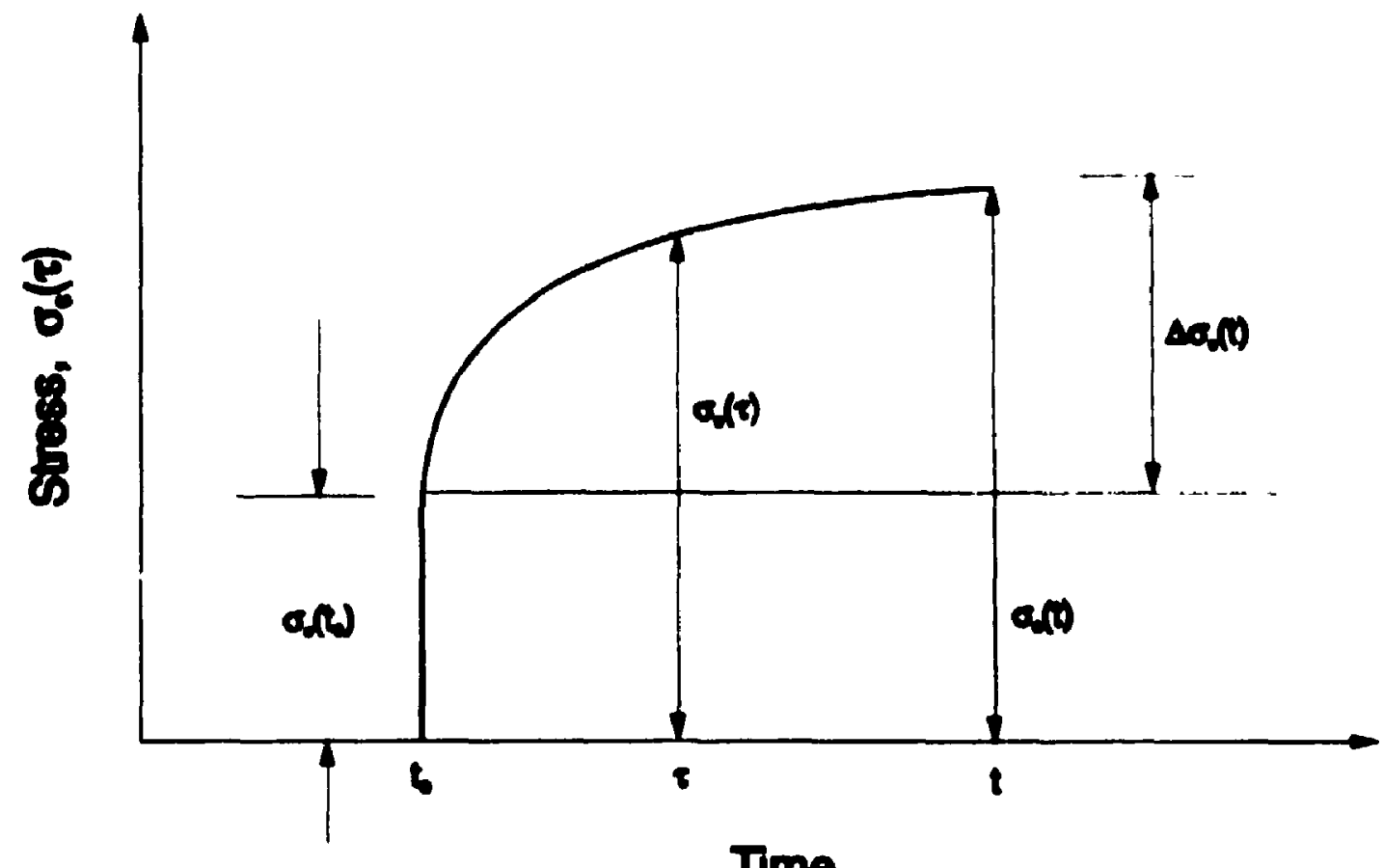

\section{Time}

Fig. 6.7 Stress varies with time $t$.

Consider a concrete member subjected to a variable stress during the period of $t_{0}$ to $t$ as shown in Fig. 5.9a. Divide the period $\left(t_{0}-t\right)$ into time intervals as shown in Fig. 5.9b. $t_{j-1 / 2}, t_{j}$ and $t_{j+1 / 2}$ are the time at the start, the middle and the end of the $j$ th interval, respectively. The strain at the end of the ith interval can be calculated by Eq. (5.4.16)

$$
\epsilon_{c}\left(t_{i+1 / 2}\right)=\sum_{j=1}^{i}\left[\left(\Delta \sigma_{c}\right), \frac{1+\varphi\left(t_{1+1 / 2}, t_{j}\right)}{E_{c}\left(t_{j}\right)}\right]+\epsilon_{c s}\left(t_{1+1 / 2}, t_{0}\right)
$$

or by separating the last term of the summation, the alternative form can be obtained

$$
\epsilon_{c}\left(t_{i+1 / 2}\right)=\left(\Delta \sigma_{c}\right)_{1} \frac{1+\varphi\left(t_{i+1 / 2}, t_{i}\right)}{E_{c}\left(t_{1}\right)}+\sum_{j=1}^{1-1}\left[\left(\Delta \sigma_{c}\right)_{j} \frac{1+\varphi\left(t_{i+1 / 2}, t_{j}\right)}{E_{c}\left(t_{j}\right)}\right]+\epsilon_{c o}\left(t_{i+1 / 2}, t_{0}\right)
$$

When the stre $\mathrm{n}$ is known, the stress increment at ith interval can be found from 


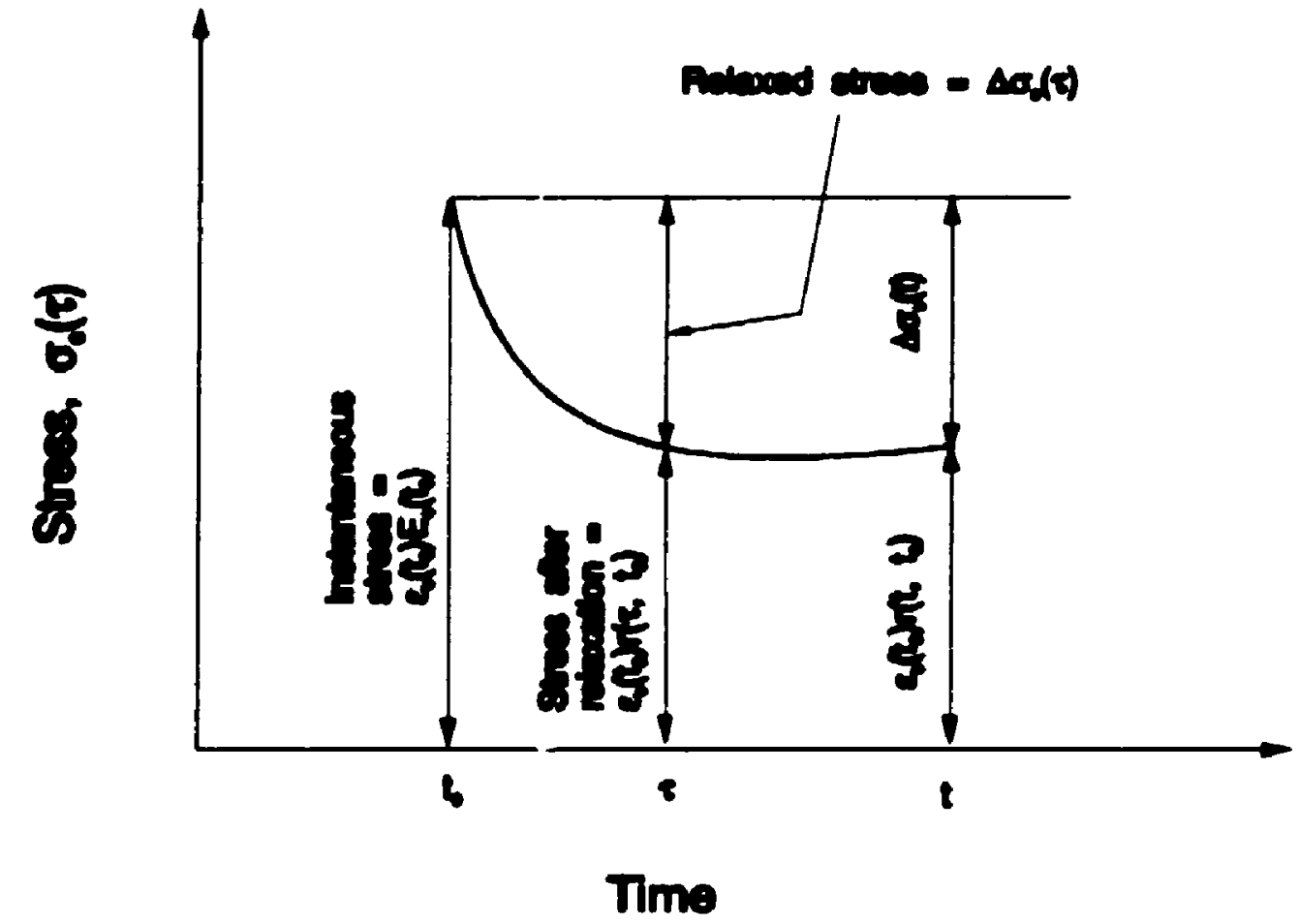

Fig. 5.8 Phenomenon of relaxation.

Eq. (5.4.19) as follows:

$$
\left(\Delta \sigma_{c}\right)_{1}=\frac{E_{c}\left(t_{i}\right)}{1+\varphi\left(t_{i+1 / 2}, t_{i}\right)}\left[\epsilon_{c}\left(t_{i+1 / 2}\right)-\epsilon_{c o}\left(t_{i+1 / 2}, t_{0}\right)-\sum_{j=1}^{i-1}\left(\left(\Delta \sigma_{c}\right), \frac{1+\varphi\left(t_{i+1 / 2}, t_{j}\right)}{E_{c}\left(t_{j}\right)}\right)\right]
$$

The stress at the end of the ith interval is the summation of all the stress increments up to that time, i.e.

$$
\sigma_{c}\left(t_{i+1 / 2}\right)=\sum_{j=1}^{i}\left(\Delta \sigma_{c}\right)_{j}
$$

From the definition of the relaxation function $r\left(t, t_{0}\right)$, the strain $\epsilon_{c}$ is constant during the period $\left(t_{0}-t\right)$, which may be conveniently chosen as unity in the calculation. By neglecting the shrinkage strain in Eq. (5.4.20), it becomes

$$
\left(\Delta \sigma_{c}\right)_{i}=\frac{E_{c}\left(t_{i}\right)}{1+\varphi\left(t_{i+1 / 2}, t_{i}\right)}+\left[1-\sum_{j=1}^{i-1}\left(\left(\Delta \sigma_{c}\right), \frac{1+\varphi\left(t_{i+1 / 2}, t_{j}\right)}{E_{c}\left(t_{j}\right)}\right)\right]
$$

Substituting $\epsilon_{c}=1$ and Eq. (5.4.21) into Eq. (5.4.14), the relaxation function of 
concrete can be expressed as

$$
r\left(t_{1+1 / 2}, t_{0}\right)=\sum_{j=1}^{1}\left(\Delta \sigma_{c}\right)
$$

Now, all the functions in Eq. (5.4.17) are known. The aging coefficient can be calculated from Eq. (5.4.17)

In finite element analysis, creep and shrinkage effects can be treated as initial strains, as in the case of temperature, and the corresponding equivalent nodal forces can be calculated. These equivalent nodal forces will then be treated as externally applied loads and the same procedures as described in Sec. (3.2) may be followed.

Extending Eq. (5.4.1) to plane stress state, the free creep strains are given by following

$$
\left\{\epsilon_{c r}\right\}=\left\{\begin{array}{c}
\epsilon_{s c r} \\
\epsilon_{s c r} \\
\gamma_{e s c r}
\end{array}\right\}=\varphi\left(t, t_{0}\right)\left\{\begin{array}{c}
\epsilon_{s}\left(t_{0}\right) \\
\epsilon_{g}\left(t_{0}\right) \\
\gamma_{s s}\left(t_{0}\right)
\end{array}\right\}=\varphi\left(t, t_{0}\right)\left\{\epsilon\left(t_{0}\right)\right\}
$$

Thus, the artificial restrained stresses can be expressed as

$$
\left\{\bar{\sigma}_{c r}\right\}=\left\{\begin{array}{l}
\bar{\sigma}_{\text {scr }} \\
\bar{\sigma}_{\text {scr }} \\
\bar{\tau}_{\text {ser }}
\end{array}\right\}=-\left[\bar{E}\left(t, t_{0}\right)\right]\left\{\epsilon_{c r}\right\}=-\left[\bar{E}\left(t, t_{0}\right)\right] \varphi\left(t, t_{0}\right)\left\{\epsilon\left(t_{0}\right)\right\}
$$

where $\left[\bar{E}\left(t, t_{0}\right)\right]$ is the age-adjusted elasticity matrix which is given by

$$
\left[\bar{E}\left(t, t_{0}\right)\right]=\frac{1}{1-\nu_{z} \nu_{s}}\left[\begin{array}{ccc}
\bar{E}_{z} & \nu_{z} \bar{E}_{z} & 0 \\
\nu_{s} \bar{E}_{s} & \bar{E}_{s} & 0 \\
0 & 0 & \left(1-\nu_{z} \nu_{s}\right) G
\end{array}\right]
$$

in which $\quad \bar{E}_{z}=\frac{E_{z}\left(t_{0}\right)}{1+\chi \varphi\left(t, t_{0}\right)}$

$$
\bar{E}_{,}=\frac{E_{0}\left(t_{0}\right)}{1+\chi \varphi\left(t, t_{0}\right)}
$$

and $G=G\left(t, t_{0}\right)$ is the shear modulus at age $t ; \nu_{z}$ and $\nu$, are the Poisson's ratios in the $z$ and $s$ directions, and $\nu_{z} \bar{E}_{z}=\nu_{s} \bar{E}_{s}$. The equivalent element nodal force vector due to creep can be obtained from

$$
\left\{F_{c r}\right\}^{e}=\int_{V}[B]^{T}\left\{\bar{\sigma}_{c r}\right\} d V=\int_{-1}^{+1} \int_{0}^{+1} \int_{-1}^{+1}[B]^{T}\left\{\bar{\sigma}_{c r}\right\}|J| d \xi d \eta d \zeta
$$




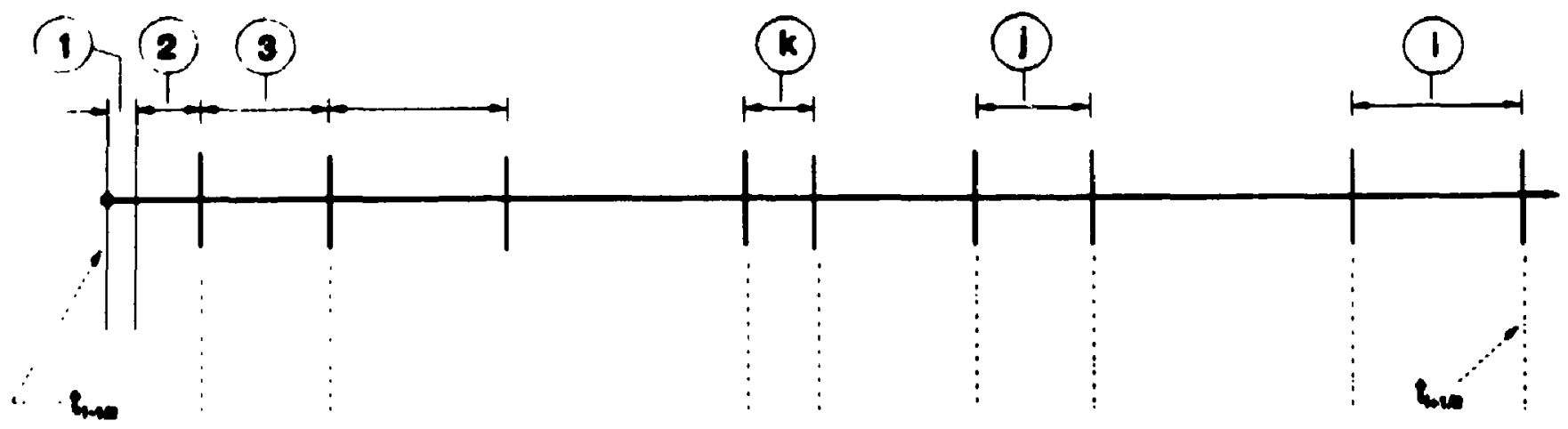

(a) Divtion of time intervale

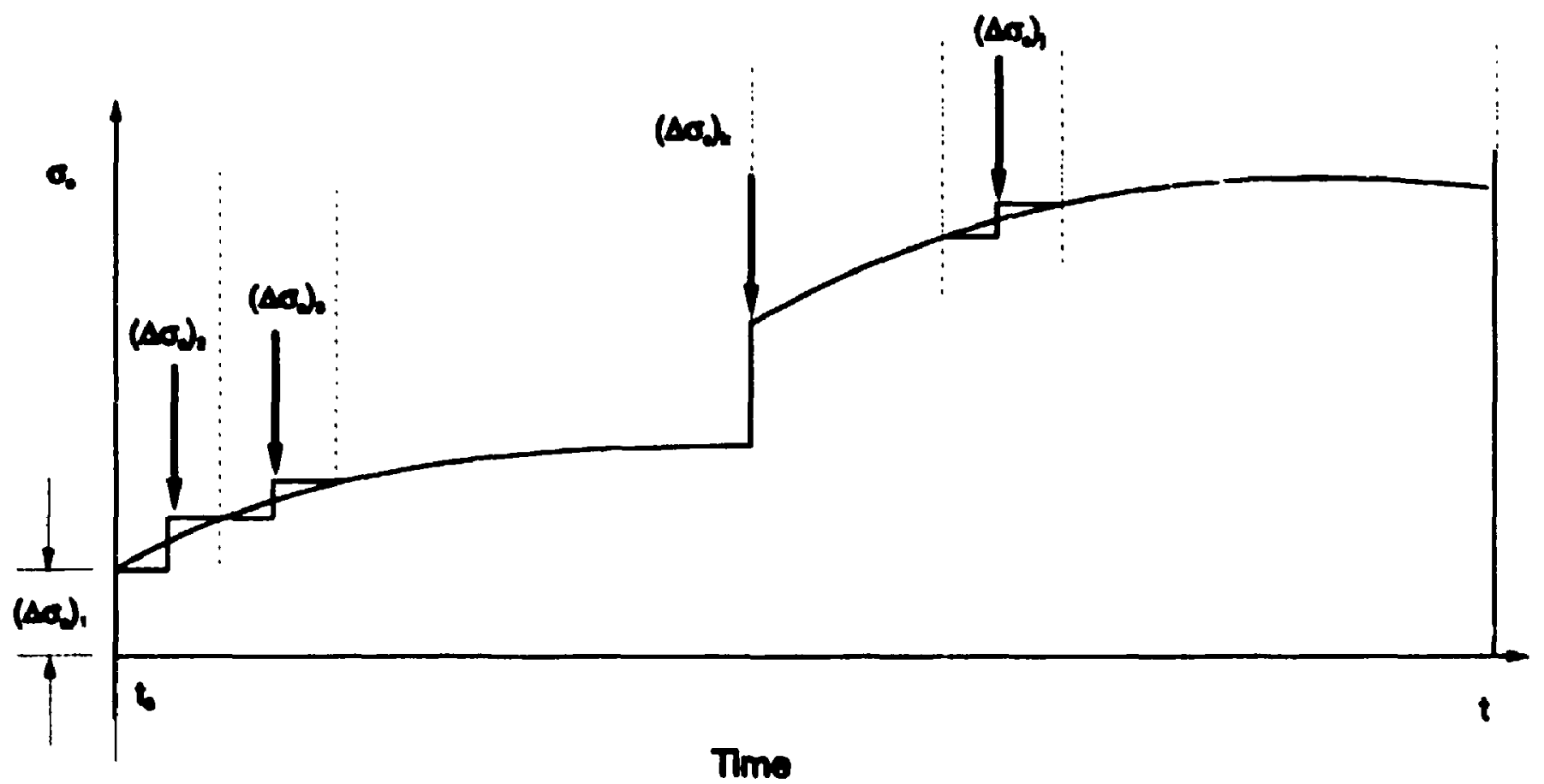

(b) Dtvision of Stress increments

Fig. 3.9 Step-by-step numerical integration. 
Additional stresses $\left\{\Delta \sigma_{\mathrm{cr}}\right\}$ will be produced by those equivalent nodal forces and the total stresses at age $t$ due to creep is the summation of the initial stresses at time $t_{0}$, the artificial restrained stresses and the additional stresses due to the equivalent nodal forces, which can be written as

$$
\left\{\sigma\left(t, t_{0}\right)\right\}=\left\{\sigma\left(t_{0}\right)\right\}+\left\{\bar{\sigma}_{c r}\right\}+\left\{\Delta \sigma_{c r}\right\}
$$

For shrinkage, similar procedures can be used and ihe free shrinkage strains for the plane stress state are given by

$$
\left\{\epsilon_{s h}\right\}=\left\{\begin{array}{c}
\epsilon_{c s}\left(t, t_{0}\right) \\
\epsilon_{c s}\left(t, t_{0}\right) \\
0
\end{array}\right\}
$$

where $\epsilon_{g h}\left(t, t_{0}\right)$ is the free shrinkage function given by Eqs. (5.4.8) and (5.4.9). The corresponding artificial restrained stresses can be found from

$$
\left\{\bar{\sigma}_{s h}\right\}=-\left[E\left(i, t_{0}\right)\right]\left\{\begin{array}{c}
\epsilon_{c s}\left(t, t_{0}\right) \\
\epsilon_{c s}\left(t, t_{0}\right) \\
0
\end{array}\right\}=\left\{\begin{array}{c}
\bar{\sigma}_{2 s h} \\
\bar{\sigma}_{s s h} \\
0
\end{array}\right\}
$$

where $\left[E\left(t, t_{0}\right)\right]$ is the elasticity matrix as defined in Eq. (4.4.13), except it is a function of time $t$; and

$$
\begin{aligned}
& \bar{\sigma}_{z s h}=-\frac{1+\nu_{z}}{1-\nu_{z} \nu_{s}} E_{z} \epsilon_{c s}\left(t, t_{0}\right) \\
& \bar{\sigma}_{s s h}=-\frac{1+\nu_{z}}{1-\nu_{z} \nu_{s}} E_{s \epsilon_{c s}\left(t, t_{0}\right)}
\end{aligned}
$$

Hence, the element equivalent nodal force vector can be calculated from

$$
\left\{F_{s h}\right\}^{e}=\int_{V}[B]^{T}\left\{\bar{\sigma}_{s h}\right\} d V=\int_{-1}^{+1} \int_{0}^{+1} \int_{-1}^{+1}[B]^{T}\left\{\bar{\sigma}_{s h}\right\}|J| d \xi d \eta d \zeta
$$

or

$$
\begin{aligned}
\left\{F_{s h}\right\}^{e}= & \int_{-1}^{+1} \int_{0}^{+1} \int_{-1}^{+1}\left(\bar{\sigma}_{z s h}\left[N^{\prime}\right]^{T}\left\{\Phi_{1}\right\}+\bar{\sigma}_{z s h}\left[N^{\prime \prime}\right]^{T}\left(\left\{\Phi_{2}\right\}-n\{\Xi\}\right)\right. \\
& \left.-\bar{\sigma}_{s s h} n[N]^{T}\{\tilde{\Xi}\}\right)|J| d \xi d \eta d \zeta
\end{aligned}
$$

Again, additional stresses $\left\{\Delta \sigma_{2 h}\right\}$ will be produced by the equivalent nodal focres due to shrinkage. The shrinkage stress at any time is obtained from the summation of 
the restrained stresses and the additional stresses produced by the equivalent nodal forces, i.e.

$$
\left\{\sigma_{s h}\right\}=\left\{\bar{\sigma}_{a h}\right\}+\left\{\Delta \sigma_{s h}\right\}
$$

The total stresses at age $t$ due to creip and shrinkage effects can be obtained by combining Eqs. (5.4.30) and (5.4.3i) as follows

$$
\left\{\sigma\left(t, t_{0}\right)\right\}=\left\{\sigma\left(t_{0}\right)\right\}+\left\{\bar{\sigma}_{c r}\right\}+\left\{\Delta \sigma_{c r}\right\}+\left\{\bar{\sigma}_{s h}\right\}+\left\{\Delta \sigma_{s h}\right\}
$$

The above procedures have been incorporated in the computer programs developed in this thesis for the analysis of multicell thin-walled box girders. Examples of the applications of the proposed method will be presented in the next chapter. 


\subsection{General}

Two computer programs, SBOXEF and CBOX (see Appendix A), have been developed to carry out the analysis of box girder structures utilizing the methods described earlier in this thesis. A number of straight, curved and haunched box girder bridges have been analysed using these two programs. Results are compared with those obtained from other numerical solutions, such as facet shell finite element, finite strip and grillage analyses, and experimental model studies. The main objective is to analyse an extensive range of bridge structures in order to evaluate the performance of the proposed finite elements and the corresponding computer programs developed in this thesis. Structural responses due to different structural actions under the eccentric loading are also studied to assist design engineers have a better physical understanding of structural behaviour of box girder structures.

The examples are divided into three groups. The first group concerns the straight single-cell and multicell, single span and multispan box girder bridges. These exum. ples serve to illustrate the accuracy and efficiency of the straight multicell box beam finite element with exact shape functions developed in Chapter 3. The second grouj) of examples, presented in Section 6.3, deals with curved, skew and haunched singlecell and multicell box girder bridges and multi-branch box girder ussemblages. The curved multicell box beam finite element model developed in Chapter 4 is used in the latter analyses. The third group, which will be discussed in Section 6.4 , consists of prestressing, temperature, creep and shrinkage problems. 


\subsection{Straight Multicell Box Girder Bridges}

Three straight box girder bridges initially considered by Maisel (1982) and one two-spine straight composite box girder considered by Zhang and Lyons (1984) are analysed by the computer program SBOXEF, which is based on the straight box beam finite element with exact shape functions developed in Chapter 3 . The results are compared with those obtained from various beam theories, facet shell finite element, finite strip and grillage analyses.

\subsubsection{Single-Cell Single Span Box Girder Bridge}

A single-cell single span concrete box girder subjucted to dead load (self-weight) and to a live load (concentrated load) of $1000 \mathrm{kN}$ at midspan over one web is shown in Fig. 6.1. The box girder is simply supported over a span of $32 \mathrm{~m}$, and has diaphragms only at the supports. The diaphragms endow the box with full torsional and distortional resistance, but with no resistance to warping at the supports. The dimensions, loading and material properties of the cross-section are shown in Fig. 6.1. Only one thin-walled beam element was used to model this box girder for normal stress calculations. In order to compare the results of the present formulation with those obtained from standard finite element shell analysis, the same box was modeled by quadrilateral facet shell elements. Due to symmetry half the box girder was used in the analysis and 144 quadrilateral elements were employed. Each element had four nodes with six degrees of freedom per node, taking account of hoth membrane (inplane) and flexural (out-of-plane) deformations. Further description of the element and the finite element program that was utilized is given by Razaqpur, Nofal and Mirza.(1989).

Some results of the two analyses are compared in Figs. 6.2 to 6.5. Figure 6.2 shows the vertical deflection curve of the box girder at the top of the loaded web under dead load and live load. The vertical deflections are generally the least sensitive of the results obtained and the present approach produced deflections comparing 
favourably with those of the shell finite element analysis.

Figure 6.3 shows the variation along the span of the longitudinal normal stress at junction $A$ obtained from various beam theories. Figures $6.4(a)$ and $6.4(b)$ show the longitudinal normal stress distribution at quarter span and at midspan. For the sake of comparison, the figures also show the same stress as predicted by conventional or engineer's beam theory. It can be seen that the proposed element yields stresses that are in close agreement with those obtained from shell finite element analyses. It can also be noticed that the effects of distortion and shear lag can be rather significant in the vicinity of the concentrated load. The difference in the maximum stress between the engineer's beam theory prediction and that of the proposed method is $21 \%$ in this case.

Figures 6.5(a) and 6.5(b) show the transverse bending stresses at midspan due to dead load and live load, respectively, which are obtained from plane frame analyses as discussed in Section 2.5.3. The results are in good agreement with those from facet shell finite element analysis. 


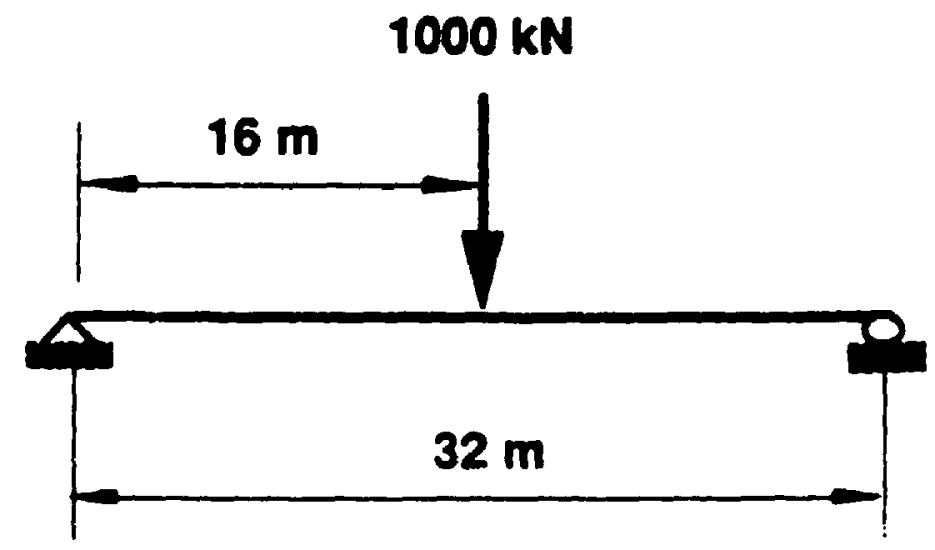

ELEVATION

$E=34500 M P a$

$G=15000 M P a$

Density $=2400 \mathrm{~kg} / \mathrm{m}^{3}$

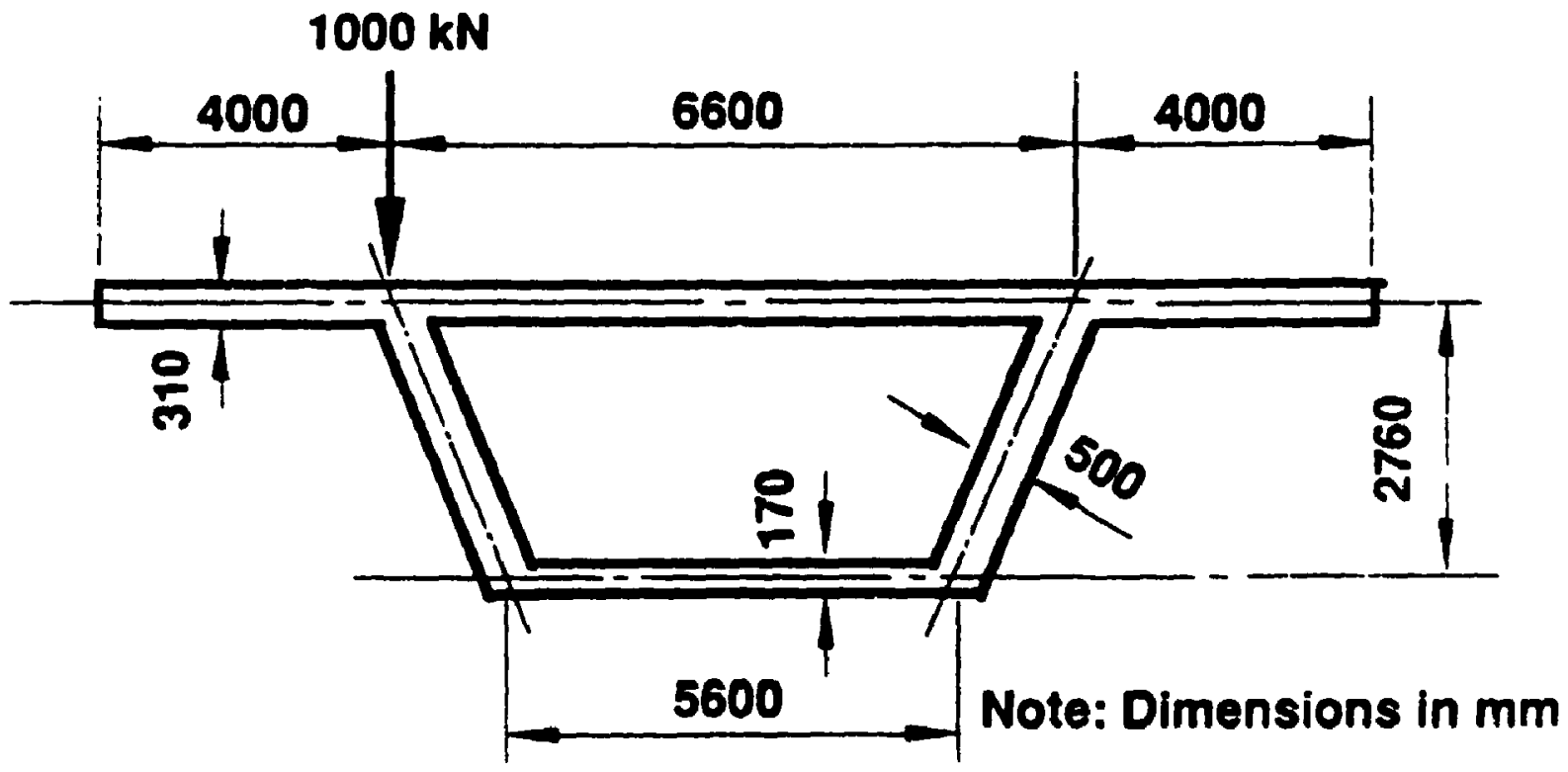

CROSS-SECTION

Fig. 6.1 Single-cell single span box girder. 


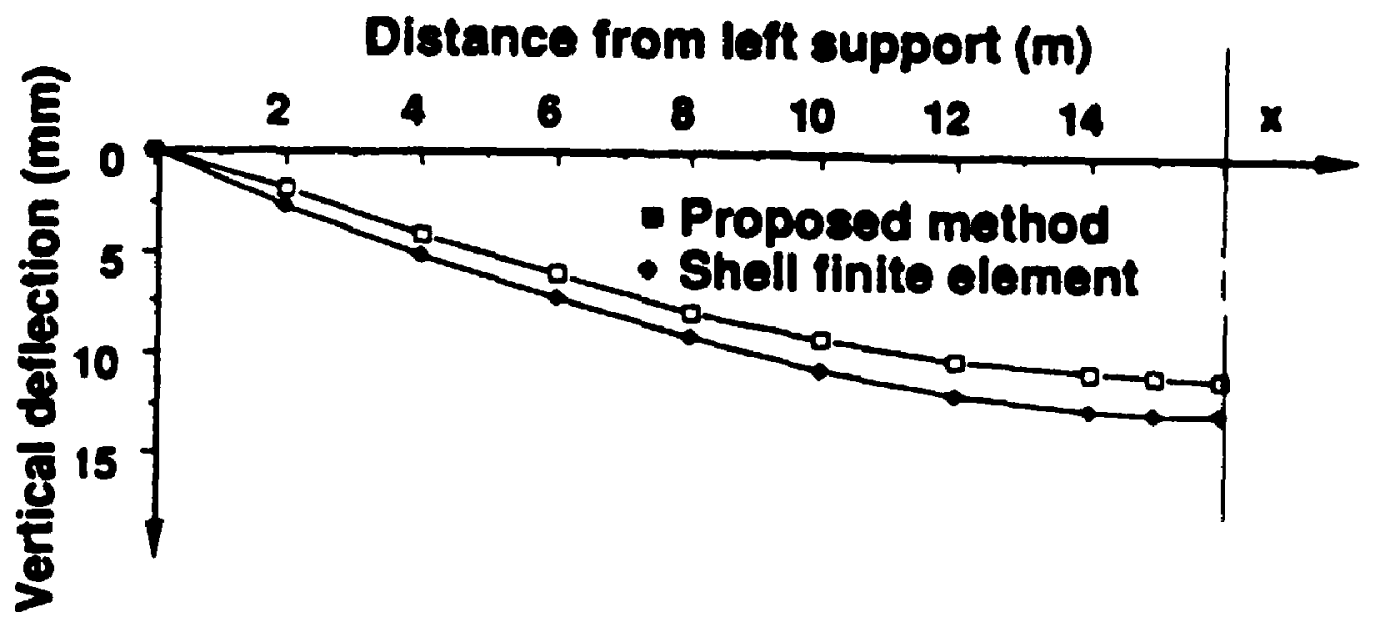

Fig. 6.2 Vertical deflection $(\mathrm{mm})$.

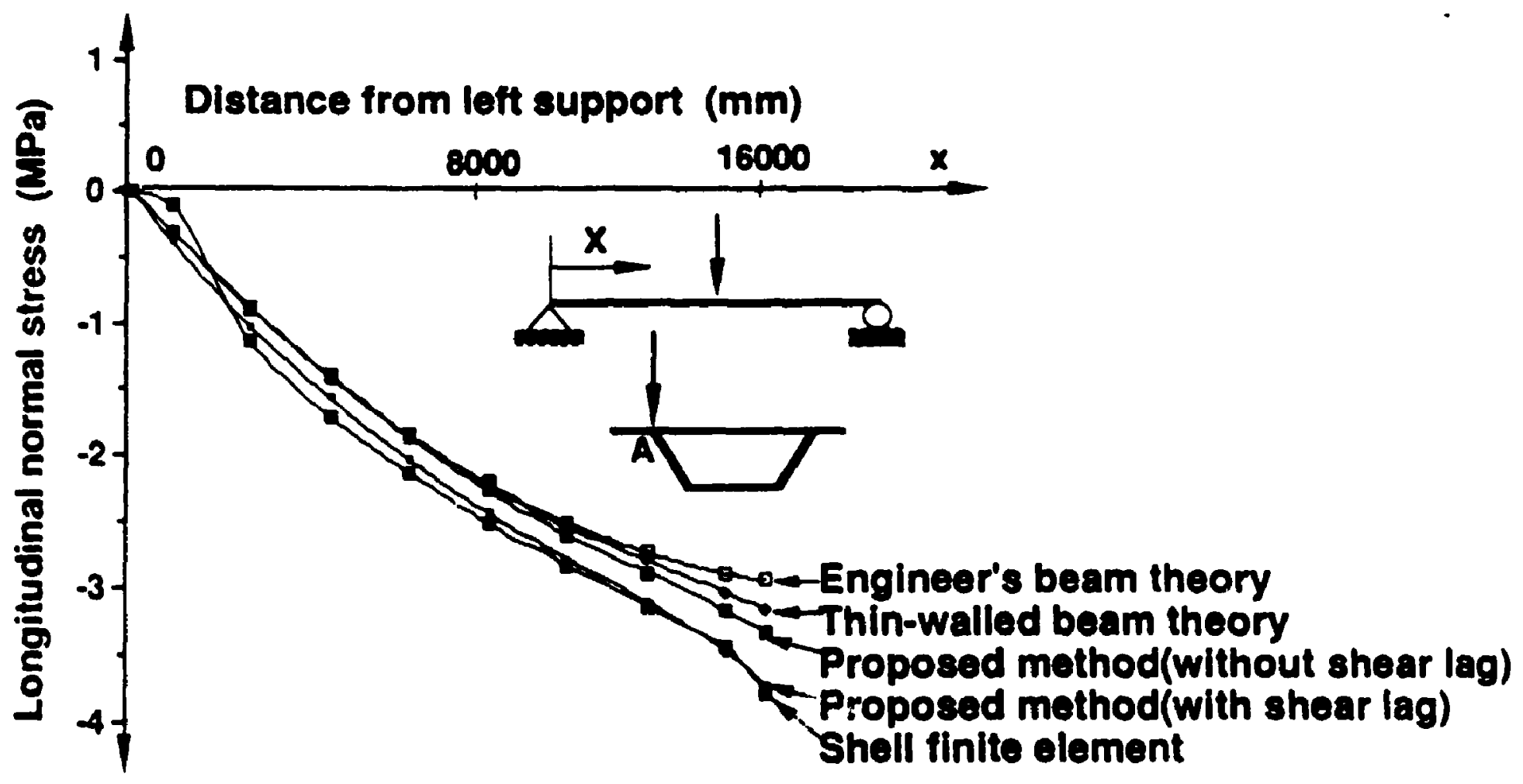

Fig. 6.3 Longitudinal normal stress along junction A (MPa). 
[-2.121] Shell finite element

-2.224 Proposed mothod

(-2.020) Engineer's beam theory

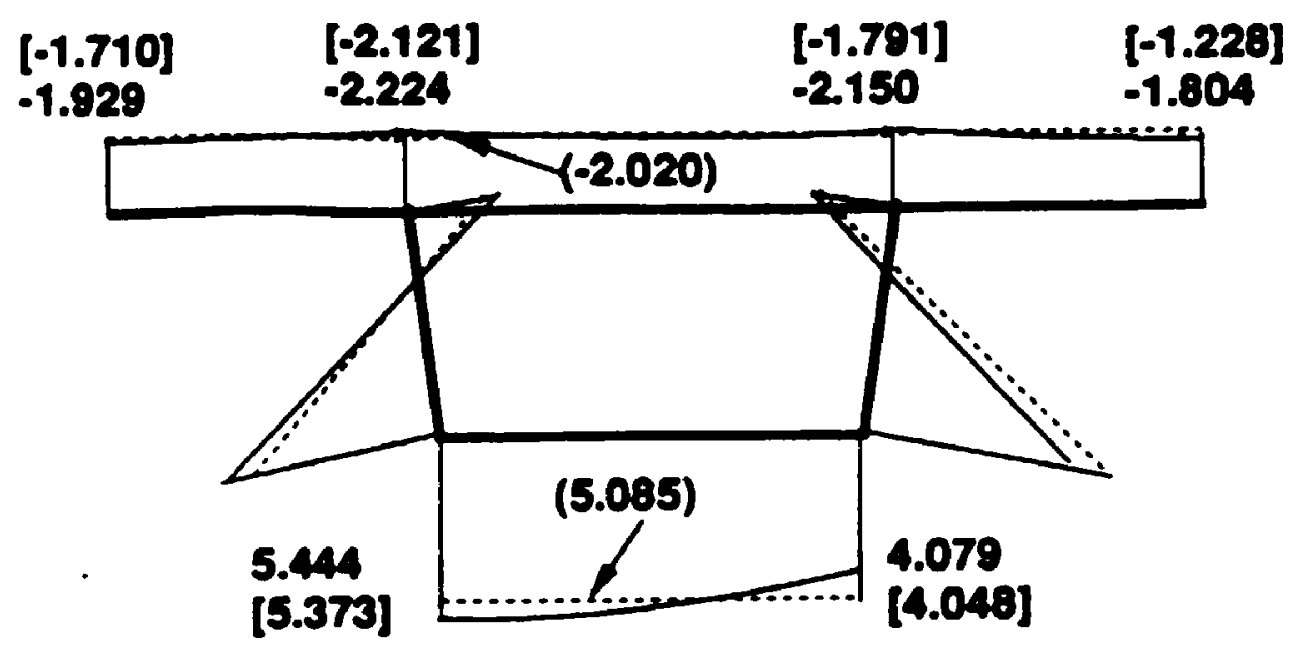

(a) Normal stress at quarter span.

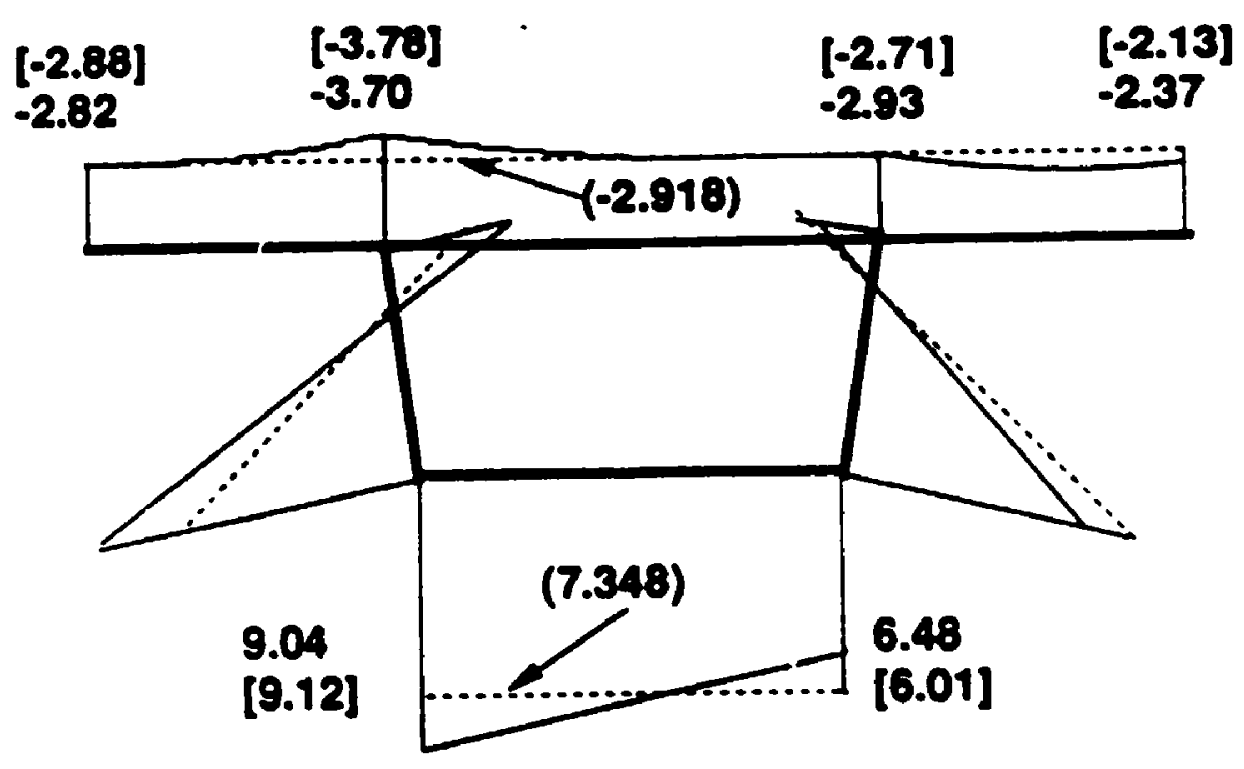

(b) Normal stress at midspan.

Fig. 6.4 Longitudinal stress distribution for single-cell box girder (MPa). 


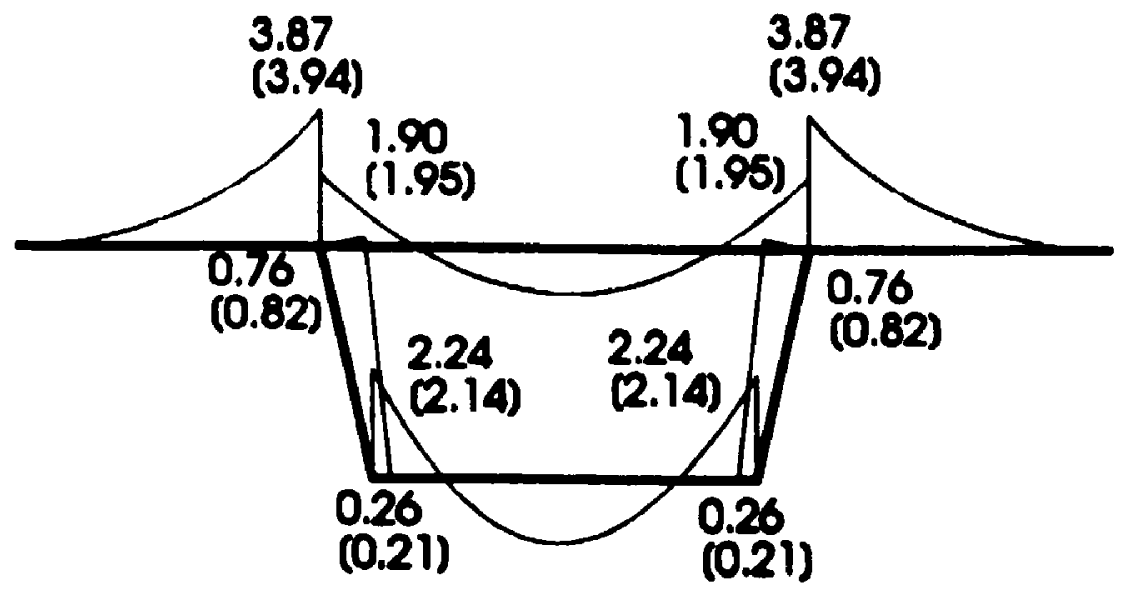

(a) Transverse bending stress due to dead load.

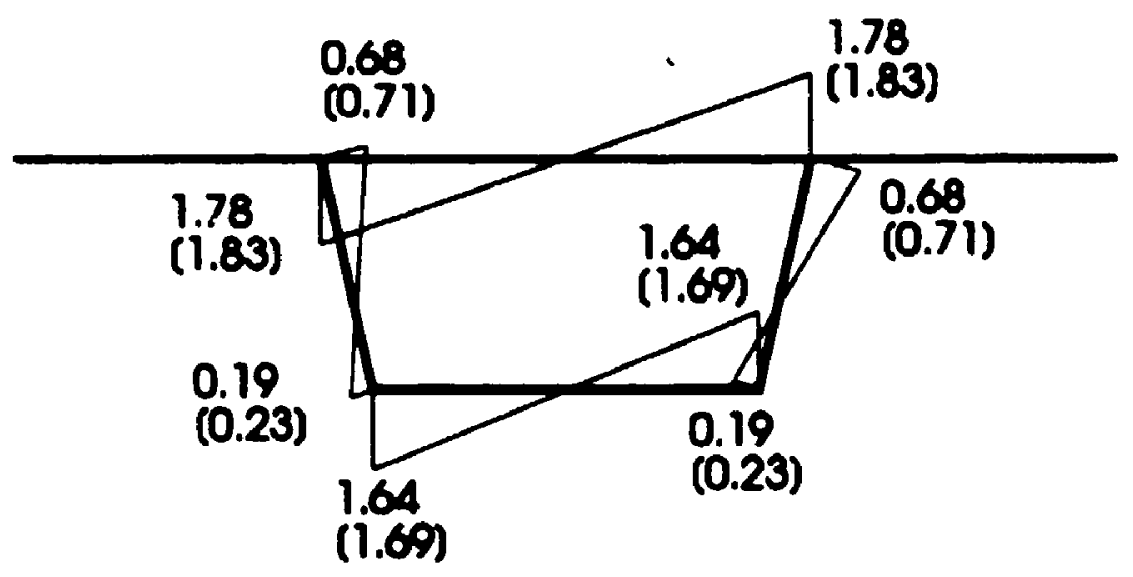

(b) Transverse bending stress due to live load.

Fig. 6.5 Transverse stress distribution for single-cell box girder (MPa). 


\subsubsection{Two-Cell Two Span Box Girder Bridge}

A two span two-cell box girder is shown in Fig. 6.6, subjected to self-weight and longitudinal symmetric concentrated loads at midspan. The box has diaphragms at all the supports and is loaded eccentrically over the outer web. The dimensions, loadings and material properties are shown in Fig. 6.6. Two thin-walled box beam elements are used in the analysis. For facet shell finite element analysis 160 elements are used to model half of the structure.

Figure 6.7 shows the deflections of the box girder at the top of the loaded web under self-weight and concentrated loads. Figure 6.8 shows the variation along the span of the longitudinal normal stress at junction A obtained from various theories. It can be seen that the stresses predicted by various beam theories are very close with those predicted by shell finite element method except at the location of concetrated loaded and intermediate supports. But in bridge structures, in order to model the vehicle load, more than one concentrated load is needed and the vehicle is moving along the bridge span. It can be concluded that shear lag and warping effects due to torsion and distortion can not be neglected in box girder bridges.

Figures 6.9(a) and 6.9(b) show the longitudinal normal stress distribution at two locations along the span. The figures also show the normal stress distribution as predicted by the conventional beam theory and by shell finite element analysis. We can observe good comparison between the sheli finite element and proposed model results. The conventional beam theory results deviate from the shell finite element results by a maximum of $38 \%$.

Figures 6.10(a) and 6.10(b) show the comparison of transverse bending stresses at midspan due to self-weight and concentrated load calculated by plane frame method and by facet shell finite element method. 


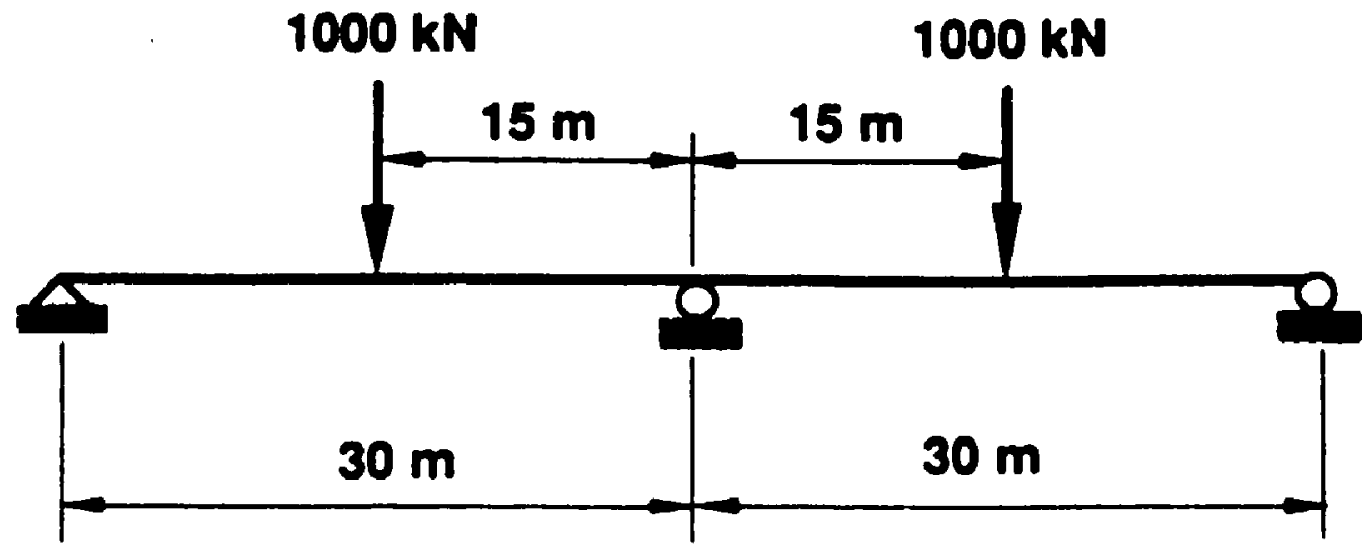

ELEVATION

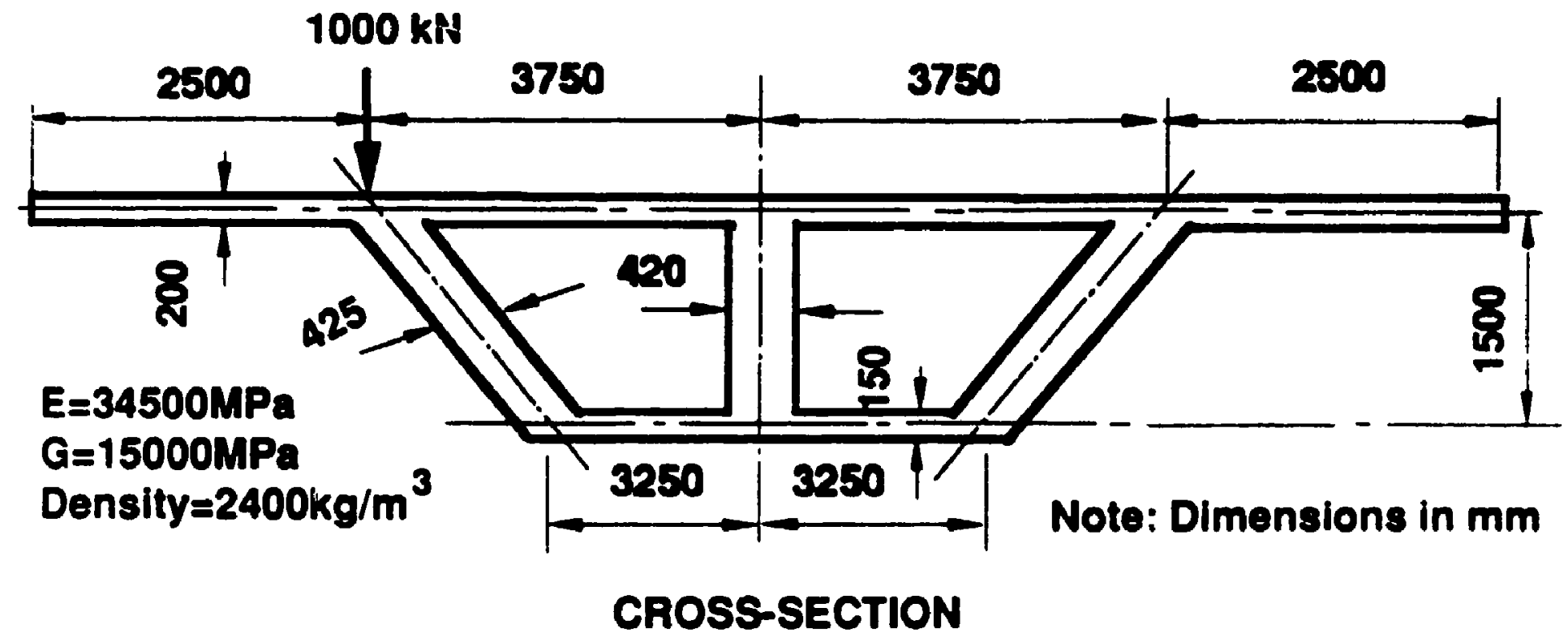

Fig. 6.6 Two-cell two span box girder. 


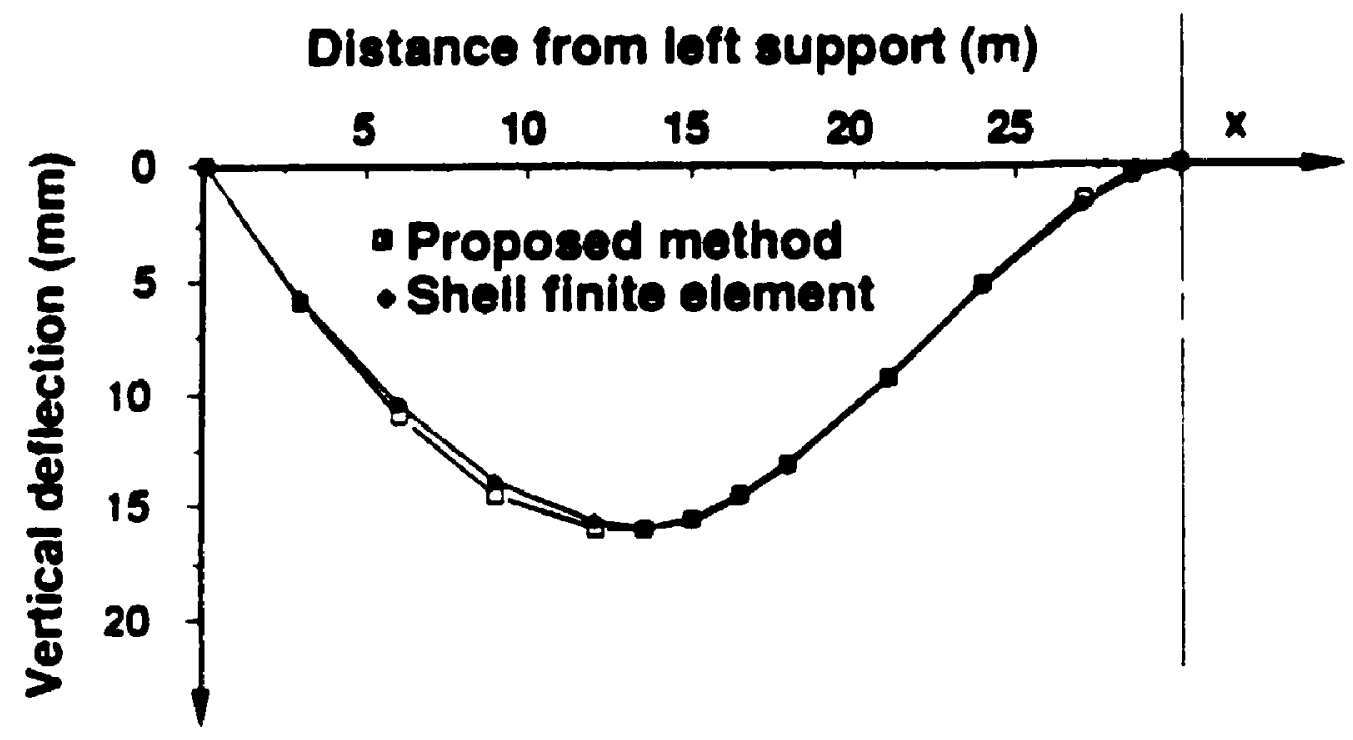

Fig. 6.7 Vertical deflection ( $\mathrm{mm}$ ).

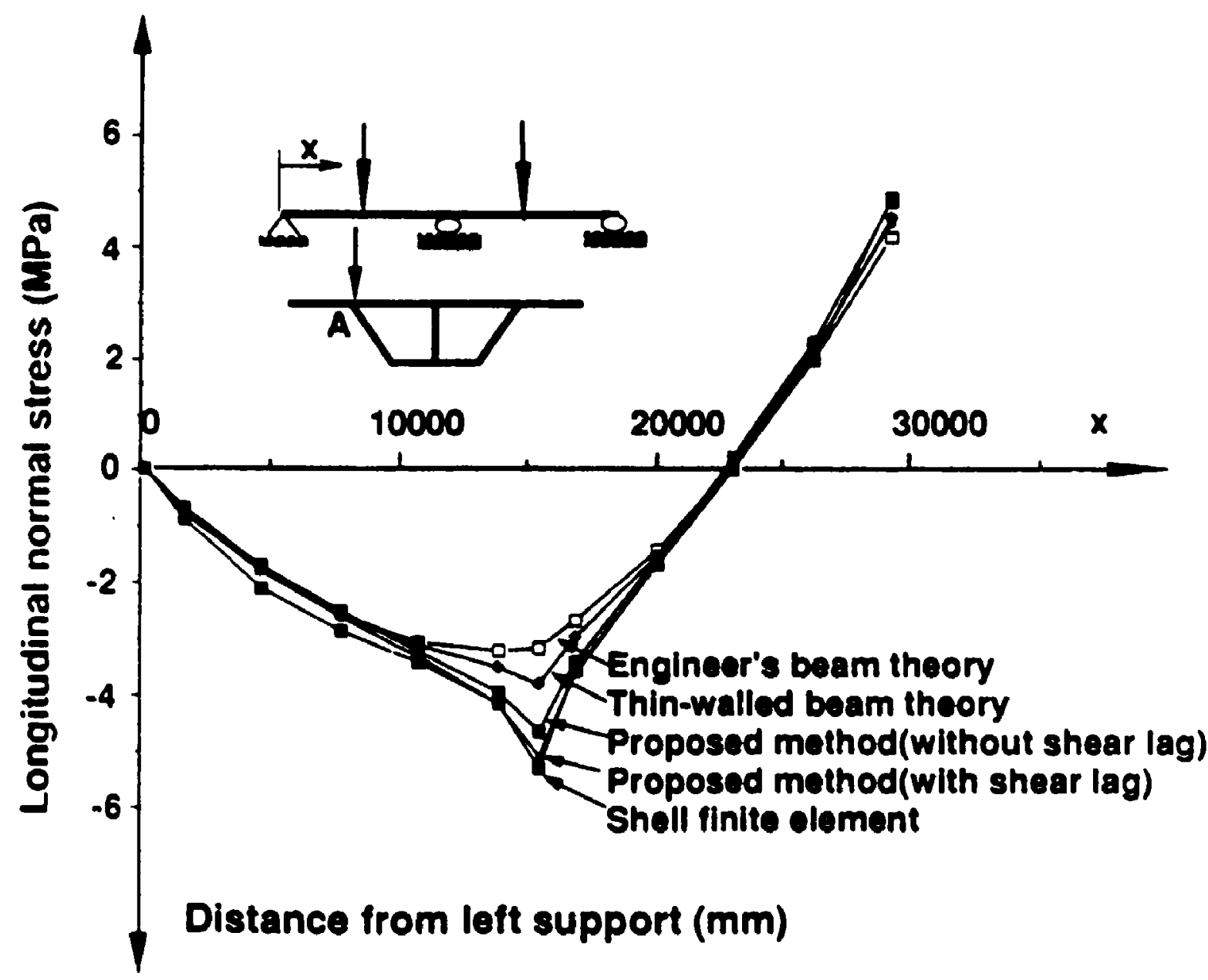

Fig. 6.8 Longitudinal normal stress along junction A (MPa). 


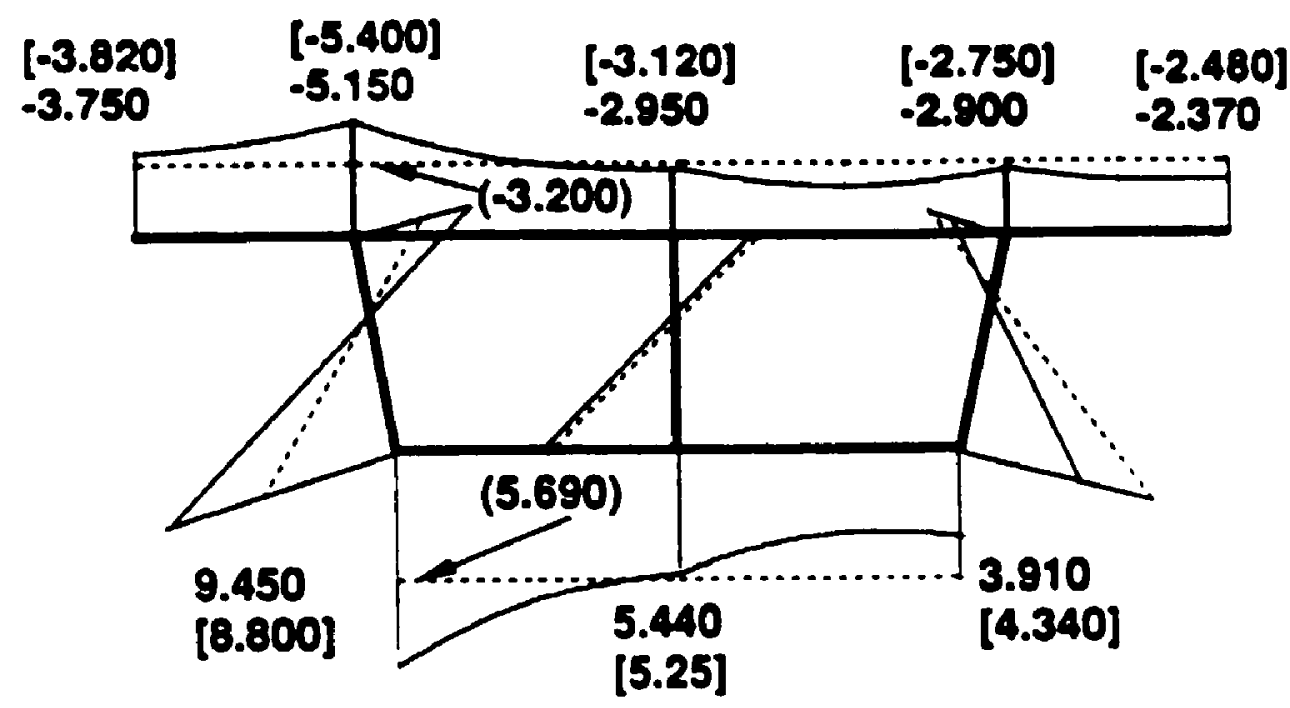

(a) Normal stress at midspan.

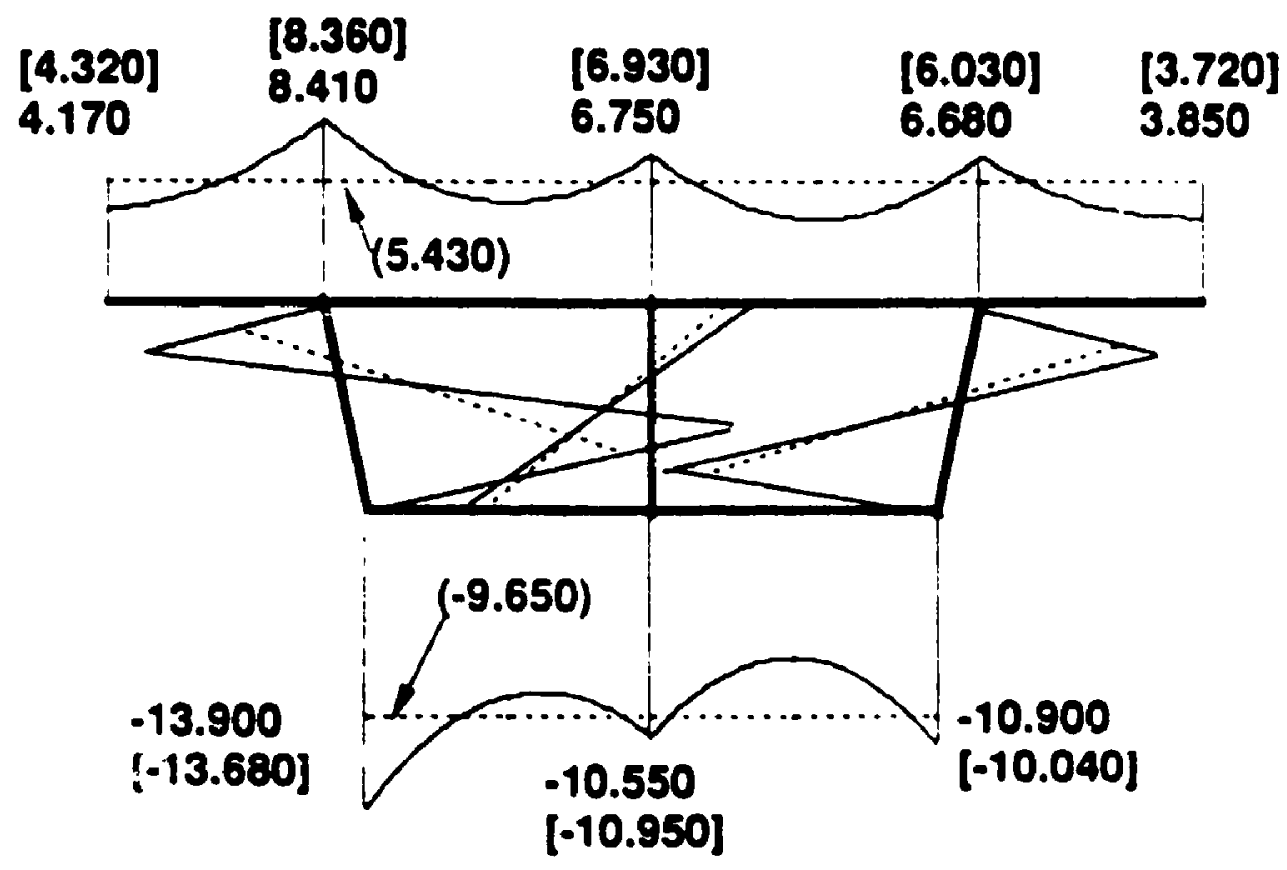

(b) Normal stress at midsupport.

Fig. 6.9 Longitudinal stress distribution for two-cell box girder (MPa). 


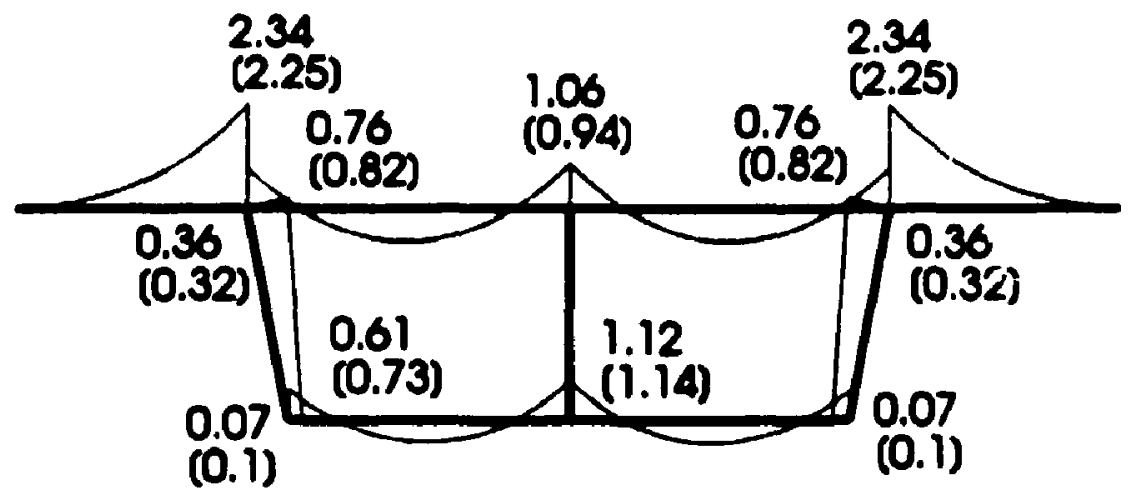

(a) Transverse bending stress due to dead load.

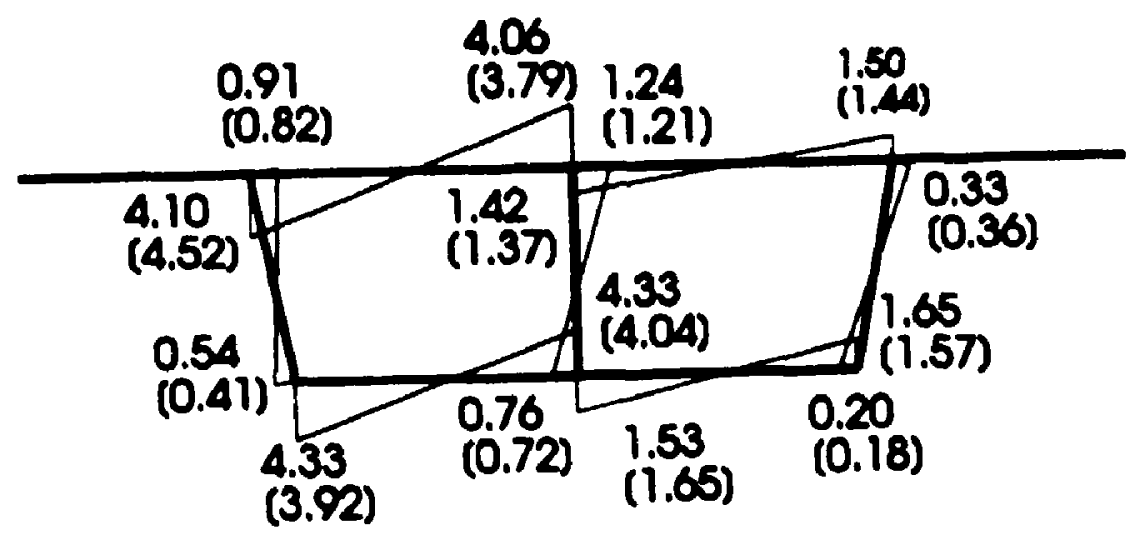

(b) Transverse bending stress due to live load.

Fig. 6.10 Transverse stress distribution for two-cell box girder (MPa). 


\subsubsection{Three-Cell Single Span Box Girder Bridge}

Consider a three-cell single span sinply supported box girder with diaphragins only at the ends, where there is full torsional and distortional restraint. The span is $50 \mathrm{~m}$ and the diaphragms are assumed to offer no resistance to warping. At midspan, there is a live point load of $1000 \mathrm{kN}$ over an outer web. Figure 6.11 shows the geometry and loading. The dimensions of the cross section are a simplified version of those from Western Avenue Extension, London, England (Maisel 1982).

Only one thin-walled box beam element is used to model this box girder. In order to compare with the load distribution method (or simplified method) given by the Ontario Highway Bridge Design Code (OHBDC 1983), grillage analysis (Jaeger and Bakht 1982) which is the theoretical bases of load distribution method is carried out. For grillage analysis, space frame program STRUDL is used. Effective width ratios are used from OHBD code (1983) to consider the shear lag effects. The element mesh for grillage analysis is shown in Fig. 6.12. Results are compared with those obtained from finite strip method (Maisel 1982).

Figure 6.13 shows the deflection curve of the box girder. Figure 6.14(a) shows the longitudinal normal stress distribution at the midspan due to live load only. For the live load, the grillage analysis results deviate from the proposed model results by a maximum of $45 \%$ in this case. Figure 6.14 (b) shows the longitudinal normal stress distribution at the midspan due to combined dead load and live load. Figures 6.15(a) and 6.15(b) show the comparison of transverse bending stresses at midspan due to self-weight and live load calculated by plane frame method and by finite strip method. We can observe good comparison between the finite strip and proposed model results. The conventional beam theory results deviate from the finite strip results by a maximum of $7 \%$. 


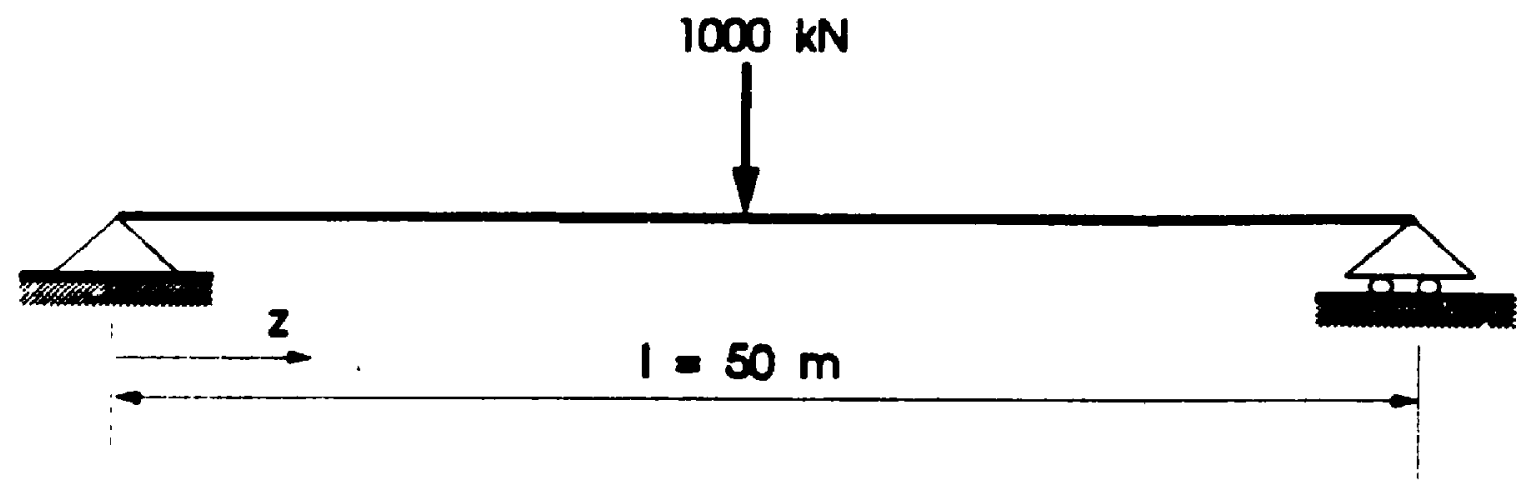

ELEVATION

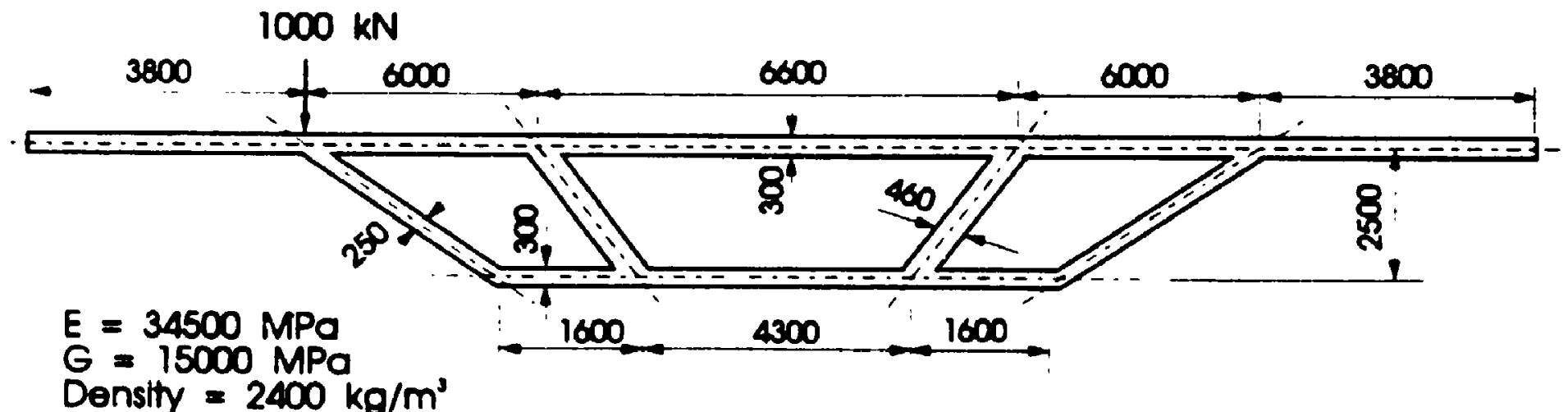

CROSS SECTION

Fig. 6.11 Three-cell single span box girder. 
Point lood

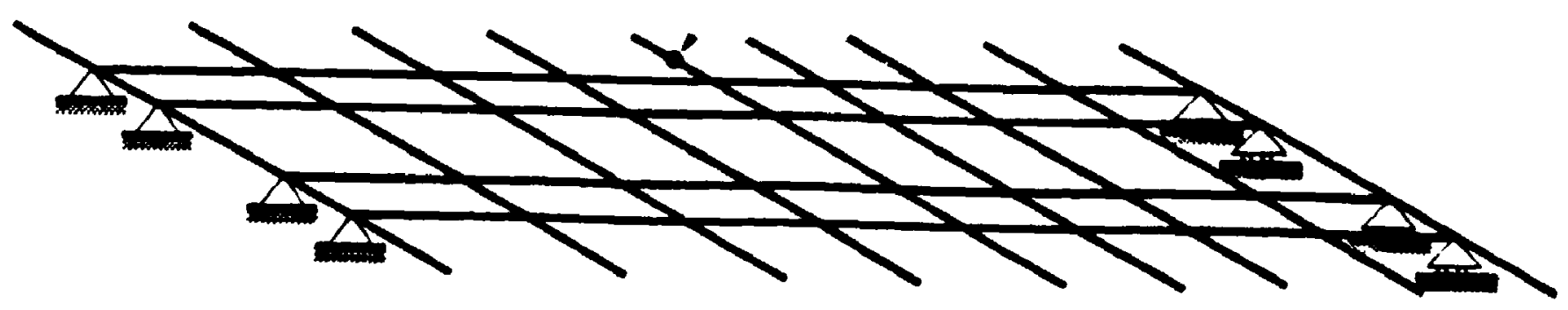

Fig. 6.12 Finite element mesh for grillage analysis.

Distance from left support (m)

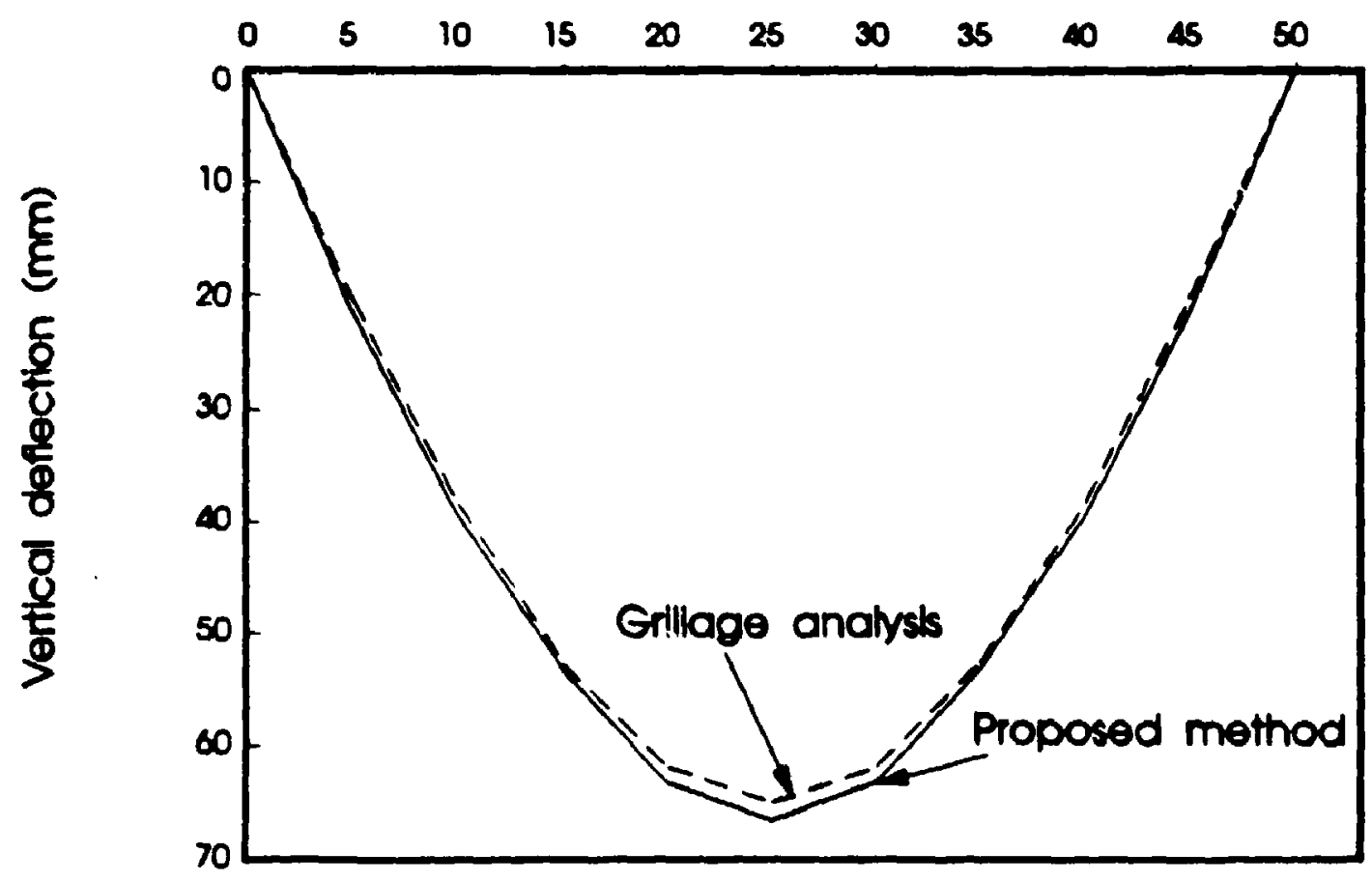

Fig. 6.13 Vertical defiection ( $\mathrm{mm}$ ). 
-1.490 Proposed method

(-1.163) Crlines entypla

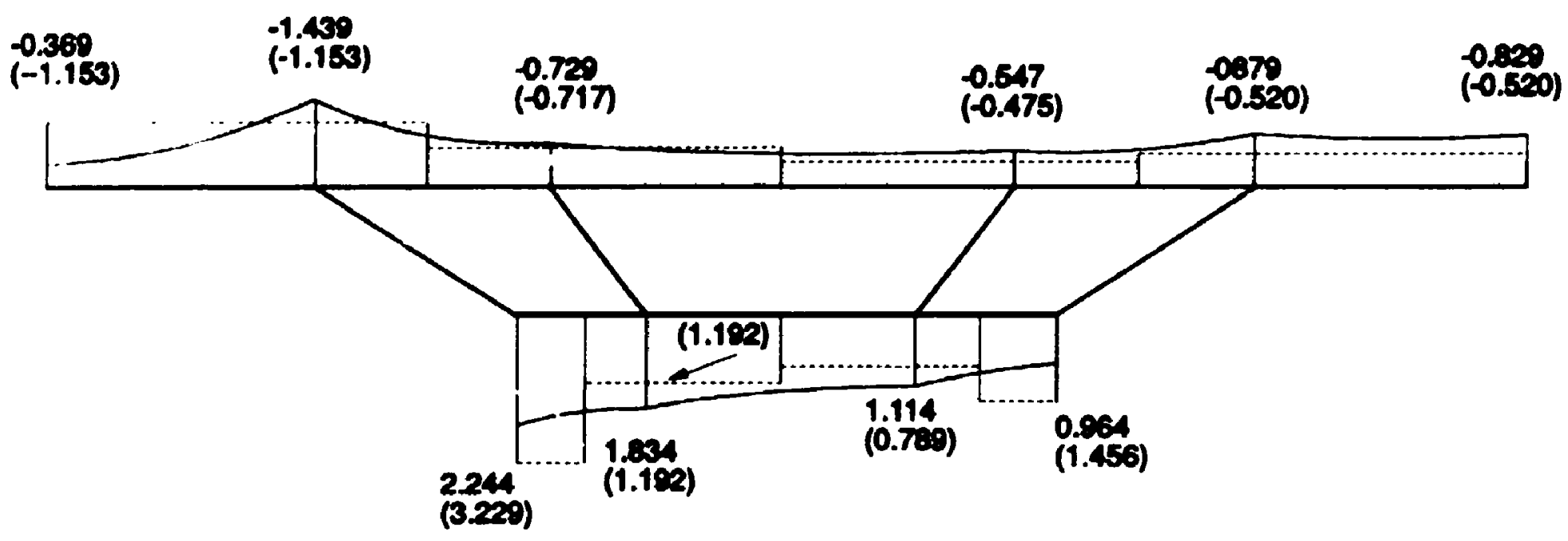

(a) Longitudinal normal stress due to live load.
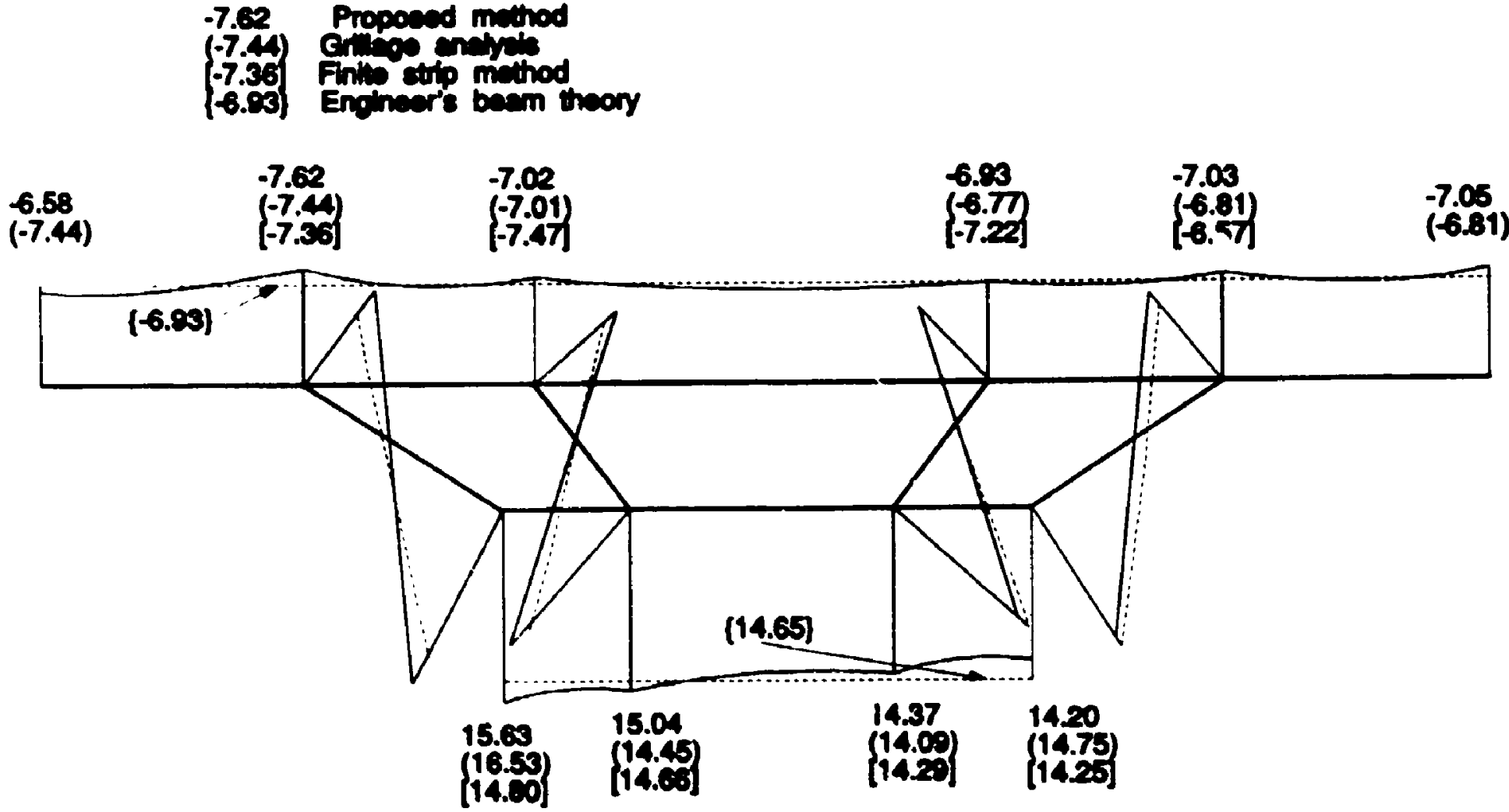

(b) Longitudinal normal stress due to combined dead and live loads.

Fig. 6.14 Longitudinal normal stress distribution at midspan (MPa). 


\subsection{Propoed mathod}

[3.47] Fintit etip mothod

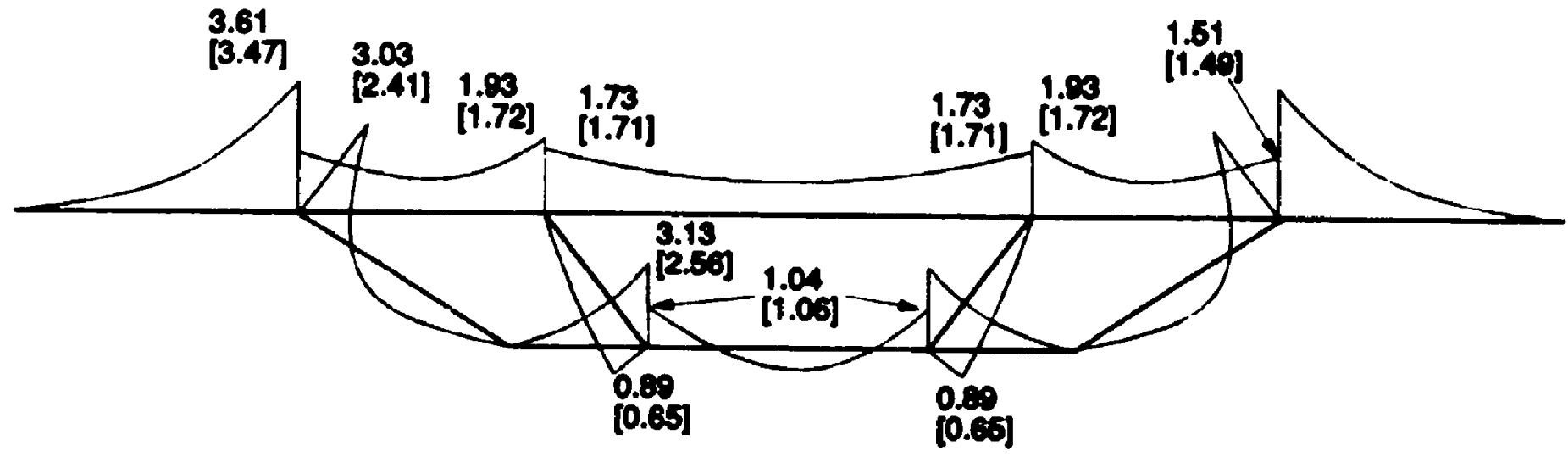

(a) Transverse bending stress due to dead load.

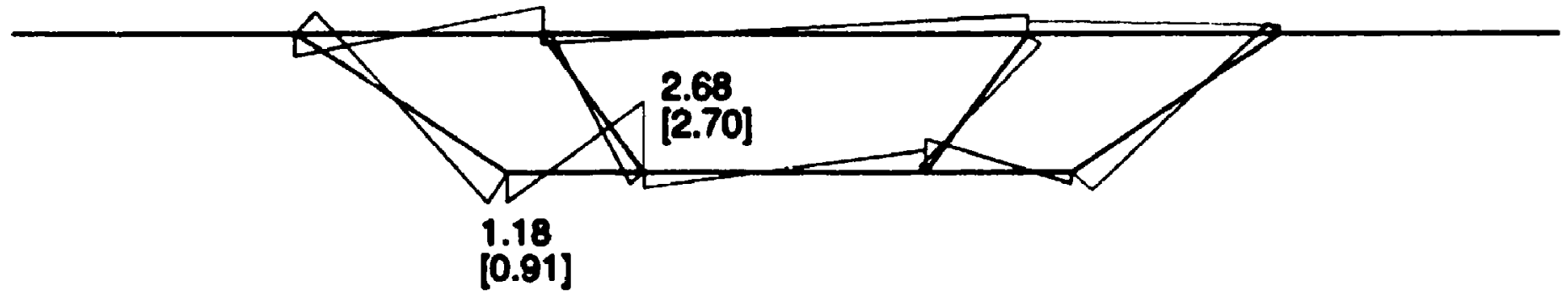

(b) Transverse bending stress due to live load.

Fig. 6.15 Transverse stress distribution for three-cell box girder (MPa). 


\subsubsection{Two-Spine Single Span Box Girder Bridge}

A simply supported composite two-spine box girder bridge with a span of $144 \mathrm{ft}$ $(43.93 \mathrm{~m})$, initially discussed by Zhang and Lyons (1984), was analysed. The cross section consists of two open-topped steel boxes with a composite reinforced concrete deck. The dimensions are shown in Fig. 6.16. A concentrated load of 1000lb (4.45 $k N$ ) is applied above an outer web at midspan. The modulus of elasticity for the steel is $3.0 \times 10^{7} \mathrm{psi}(207000 \mathrm{MPa})$, and for concrete is $40 \times 10^{6} \mathrm{psi}(27600 \mathrm{MPa})$. Poisson's ratio is taken as 0.3 for steel, and 0.2 for concrete.

Only one element is used in the present analysis. Three dimensional shell finite element and thin-walled beam grillage analyses have also been carried out by $\mathbf{Z h a n g}$ and Lyons (1984). In shell finite element analysis, 264 elements were used to model the structure, while 16 thin-walled beam elements for each bc $x$ in longitudinal direction were used in grillage analysis. Figure 6.17 shows the deflection for the concrete deck at midspan. Figure 6.18 shows the longituainal normal stress distribution at midspan section. The results are compared with those obtained from shell finite element and grillage analyses. It can be seen that the proposed method yields better results than thin-walled box beam grillage analysis compared with shell finite element method. The maximum difference between the proposed method and shell finite element analysis is $9 \%$. 
$1000 \mathrm{lb}$

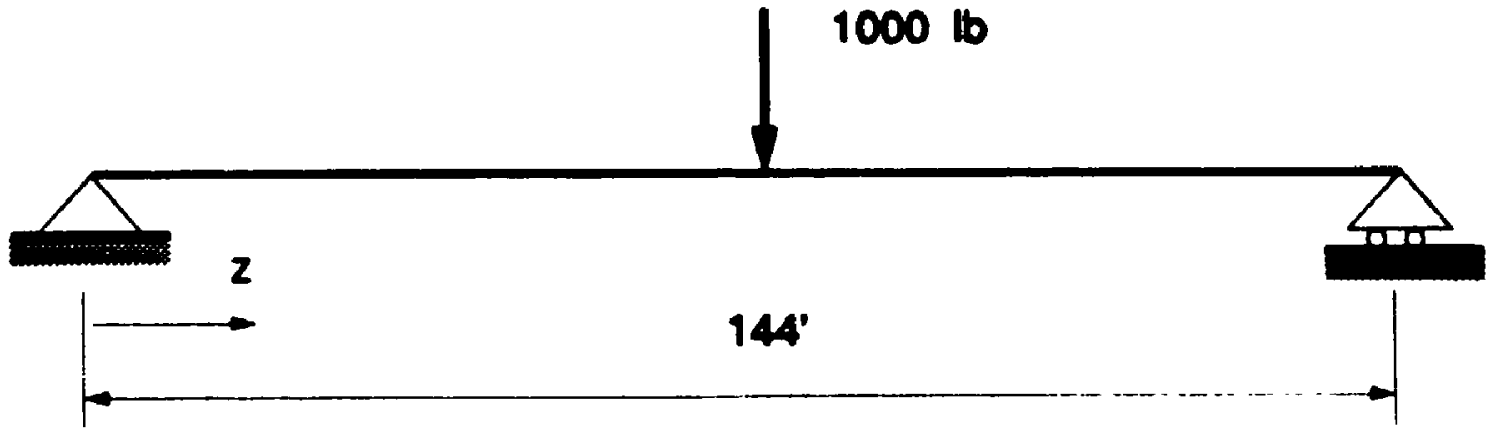

(a) ELEVATION

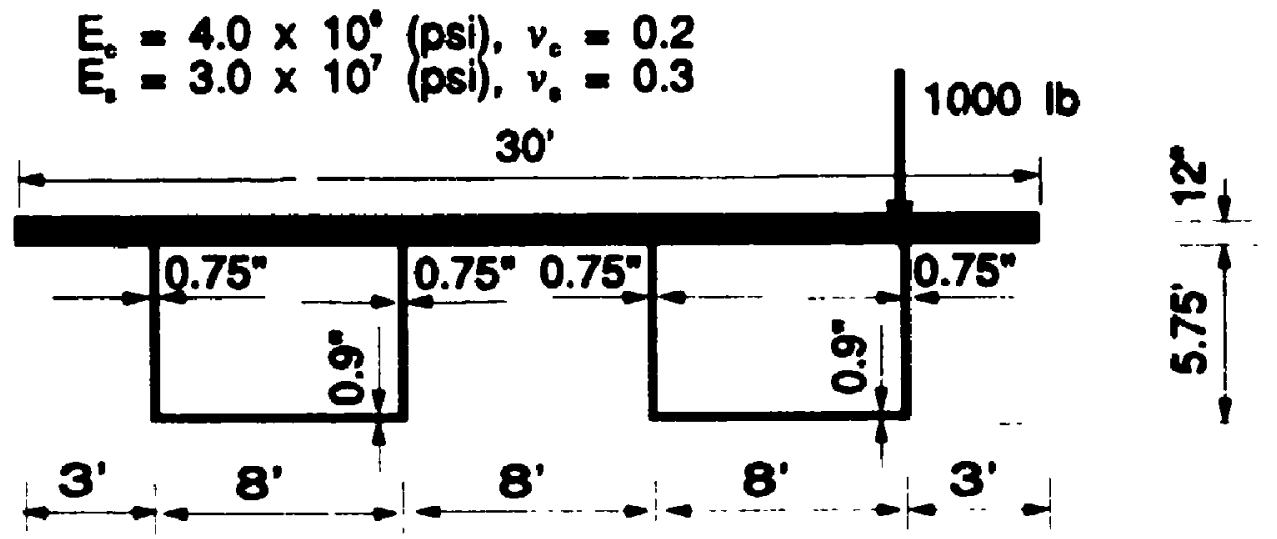

(b) CROSS SECTION

Fig. 6.16 Two-spine single span box girder. 
0.320 Propoend mothod

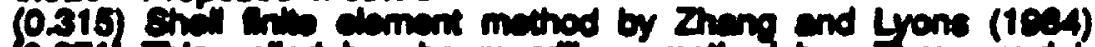

[0.271] Thinwed box bem grings monod by zhang and Lyone (1994)

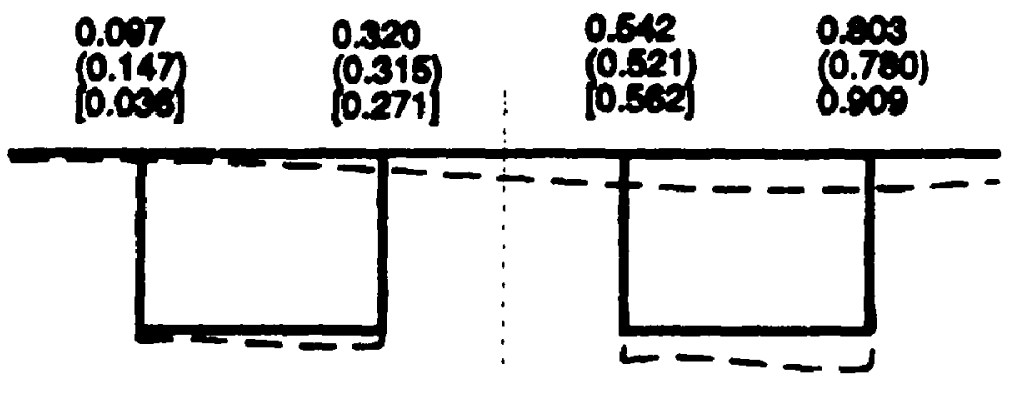

Fig. 6.17 Deflection at midspan $\left(\times 10^{-2}\right.$ in.).

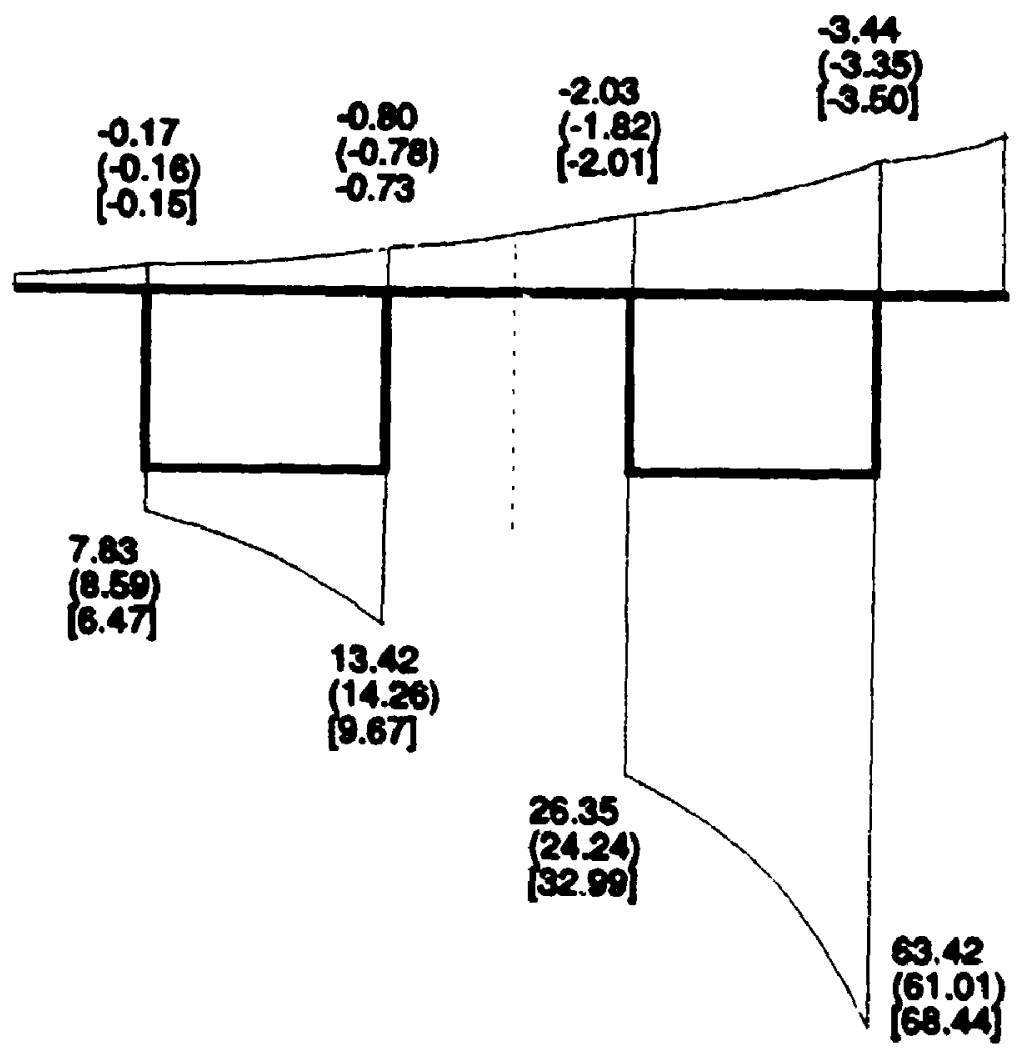

Fig. 6.18 Longitudinal normal stress distribution at midspan (psi). 


\subsection{Curved Box Girder Bridges}

Some examples for various box girder bridges are solved by the computer program CBOX to demonstrate the accuracy and versatility of the thin-walled curved box beam finite element developed in Chapter 4. The results obtained for the examples are compared with those obtained from experimental model studies and alternative numerical methods by other researchers.

\subsubsection{Single-Cell Straight Box Girder Bridges}

In order to test the model developed in Chapter 4 and the corresponding computer program CBOX, a simply supported single-cell straight box girder model with cantilever slabs is first examined. The upan of the model is $60 \mathrm{in} .(1524 \mathrm{~mm})$ and the cross section is shown in Fig. 6.19. An asymmetrical point load of $224 \mathrm{lb}$ (0.9968 $\mathrm{kN}$ ) producing torsional effects applied at midspan above the web is considered. The same problem has also been considered by Sawko and Cope (1970), and Mikkola and Paavola (1980). Since the existing results vary considerably, it is difficult to make a satisfactory comparison. Thus, we reanalyze this problem by using the fucet shell finite element analysis (Razaqpur, Nofal and Mirza 1989).

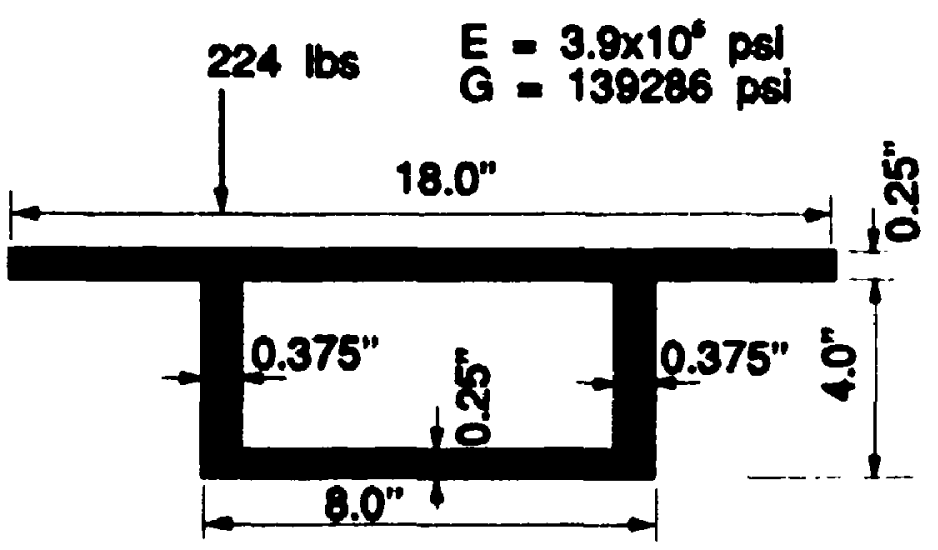

Fig. 6.19 Cross section of a straight single-cell box girder.

Twenty thin-walled box beam elements are used for the whole beam in the present study. Mikkola and Paavola (1980) analyzed the same box girder using a thirty element mesh. 


\subsection{Proposed mothod}

(0.134) Shell finlite eloment

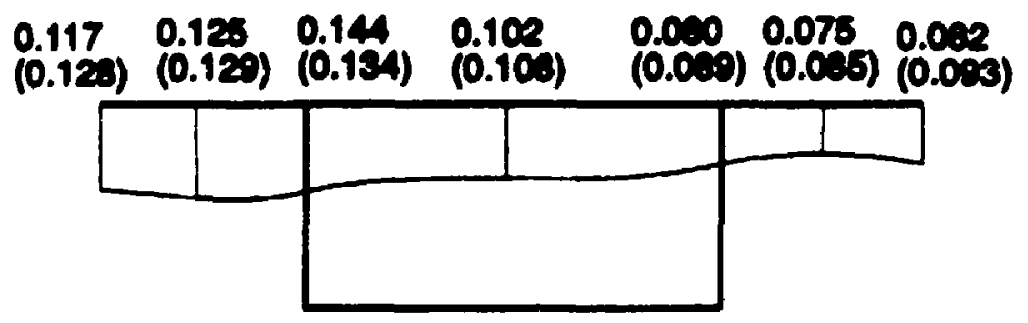

(a) Vertical deflection (in.).

321 Proposed mothod (-226) Shell innibe element

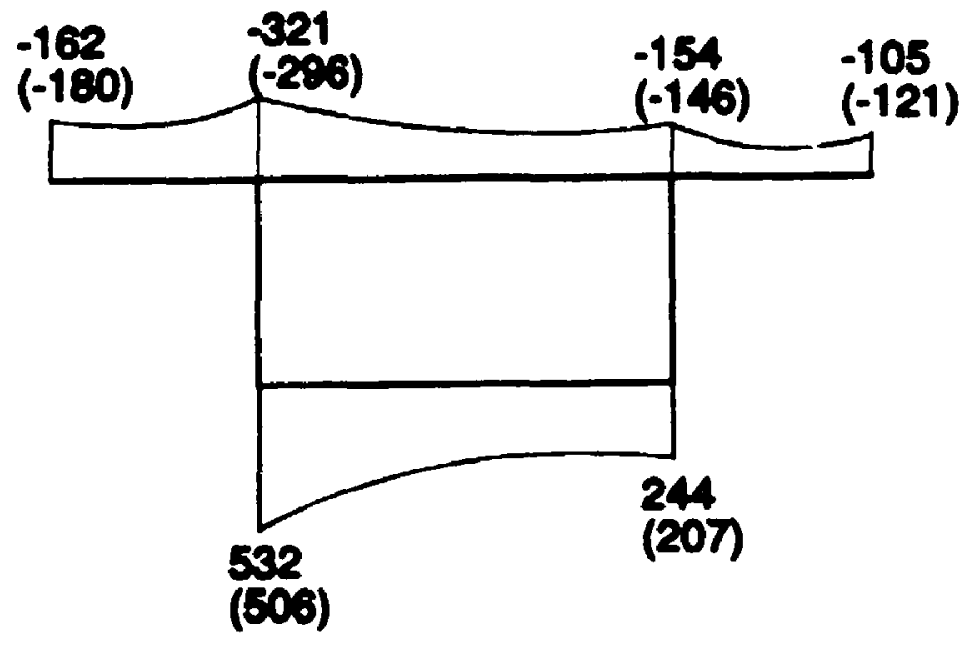

(b) Longitudinal normal stress at midspan (psi).

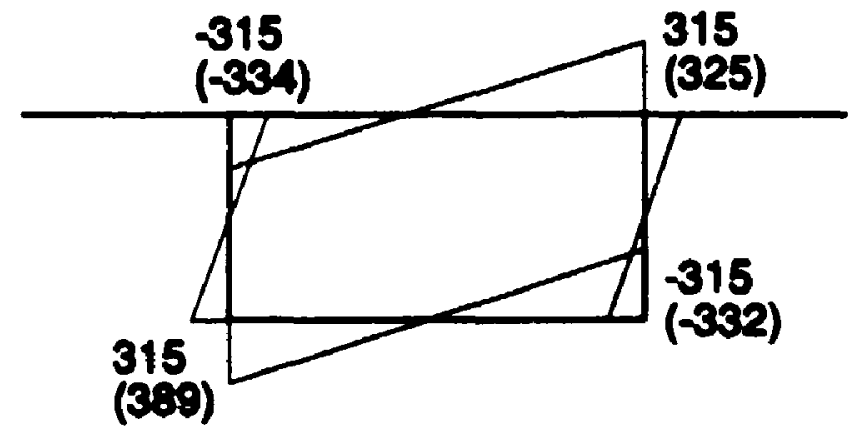

(c) Transverse bending stress (psi).

Fig. 6.20 Comparison with shell finite element method.

Figure 6.20(a) displays the deformed shape of the cross segtion at midspan. Good agreement between the present results and the facet shell finite element analysis is 
evident. The maximum difference between the results of the proposed element and those of the shell finite element is $\mathbf{8 . 5 \%}$. The longitudinal membrane streases are shown in Fig. $6.20(\mathrm{~b})$, and the transverse bending stresses at the outer surface at midspan is shown in Fig. $6.20(\mathrm{c})$. A close agreement for practical engineering purpose is evident between the results obtained from the thin-walled box beam elements and from the shell finite elements.

\subsubsection{Single-Cell Haunched Box Beams}

Two single-cell tapered box beam models studied both analytically and experimentally by Kristek (1970), are selected as further examples to verify the present work.

Kristek (1970) did the experiments on tapered celluloid box girder models with fixed ends. The models are shown in Figs. 6.21 and 6.22. The span of the first model is 24 in. $(610 \mathrm{~mm})$, and the width is 1.6 in. $(40.6 \mathrm{~mm})$, while the depth varied parabolically from $3.2 \mathrm{in}$. $(81.3 \mathrm{~mm})$ to $7.2 \mathrm{in}$. (182.9 $\mathrm{mm})$. The second beam has a span of $48 \mathrm{in}$. (1220 mm), a width of 4 in. (102 mm), and a depth which varied parabolically from $4.7 \mathrm{in} .(119 \mathrm{~mm})$ at the supports to $3.2 \mathrm{in} .(81.3 \mathrm{~mm})$ at midspan. The wall thicknesses are constant at $0.12 \mathrm{in.}(3.05 \mathrm{~mm})$ in both models. The modulus of elasticity is $500,000 \mathrm{psi}\left(3.45 \mathrm{kN} / \mathrm{mm}^{2}\right)$ and the Poisson's ratio is 0.33 .

Kristek developed a two step elastic procedure to analyze this kind of box beam. In the first step, the box is assumed to have no transverse distortion. The stiffness of the cross section for the first model is ensured by means of diagonal cross braces at regular intervals. The girder is loaded at both ends by couples, the value of which is 125 in.-lb (14.13 N-m) and the ends are fixed to prevent warping.

In the present study an analysis is carried out using 20 thin-walled elements. The variation of the longitudinal warping stresses in one of the corners is plotted in Fig. 6.23 together with results obtained by Kristek (1970).

The second step of Kristek's method consisted of analysing the box girder for transverse distortion. The girder is loaded by a pair of distributed loads along the 
diagonal of the box. The load had a constant vertical component of $0.716 \mathrm{lb} / \mathrm{in}$. (125 $\mathrm{N} / \mathrm{m}$ ) and its horizontal component varied parabolically from $0.609 \mathrm{lb} / \mathrm{in}$. (106.5 $N / \mathrm{m})$ at the supports to $0.695 \mathrm{lb} / \mathrm{in} .(156.6 \mathrm{~N} / \mathrm{m})$ at midspan.

Ten elements for each half of the beam are used in the present solution. Bazant and El Nimeiri (1974) also used the same models as illustrative examples. The vertical deflections at a corner point for the various methods are given in Table 6.1, together with Kristek's experimental results. Graphical comparisons are also shown in Fig 6.24. Agreement is found to be good among the various methods.

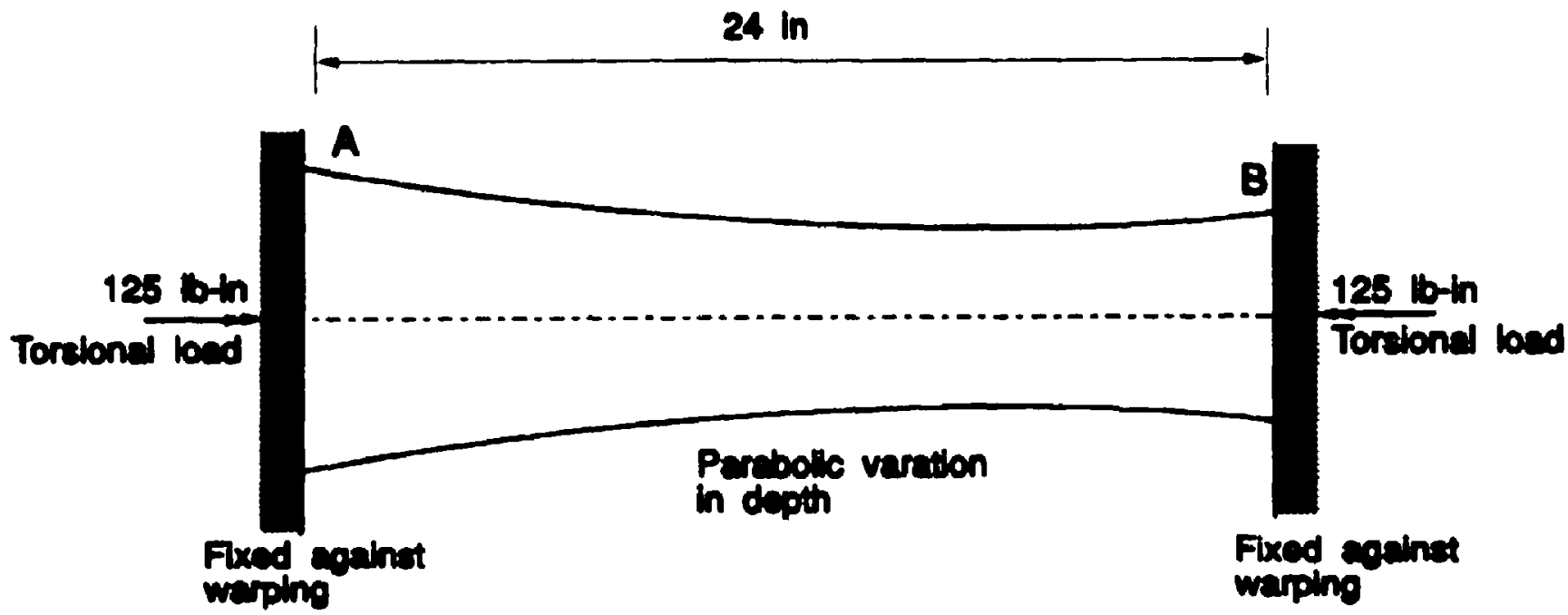

Elovation

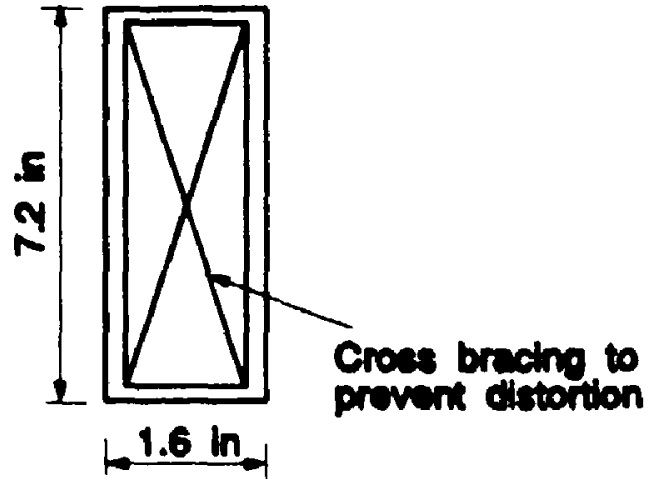

Croses excion at ' $A$ '

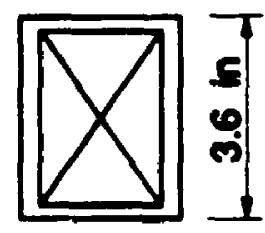

$\stackrel{1.6 \mathrm{ln}}{\longrightarrow}$ waping

Cross section at 'B'

Fig. 6.21 Experimental model by Kristek to check first of his analysis method. 

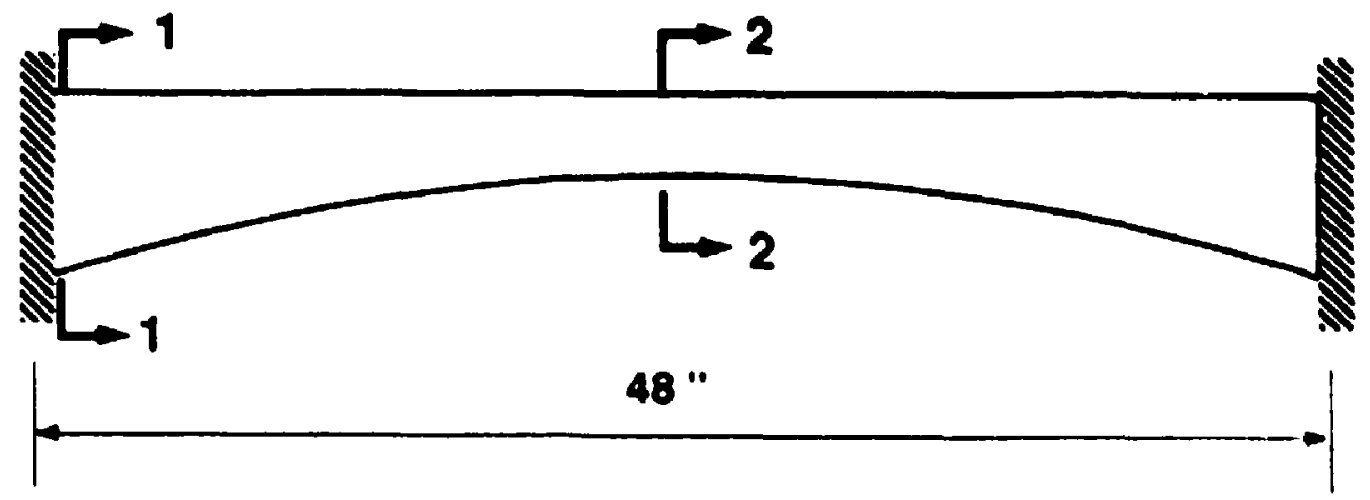

$0.716 \mathrm{lb} / \mathrm{in}$

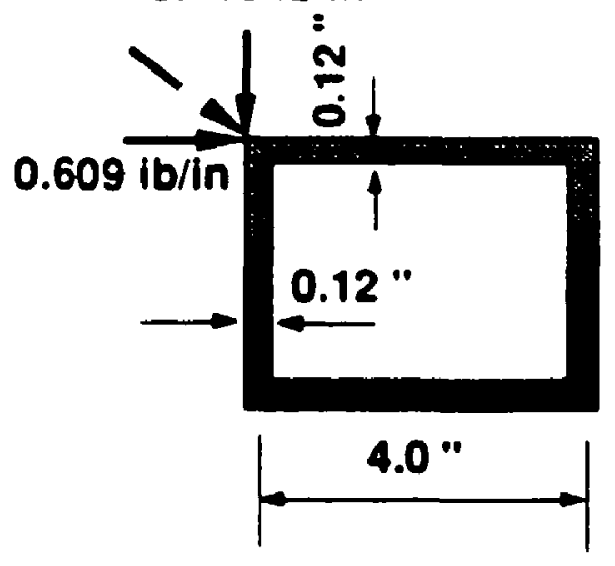

Section 1-1

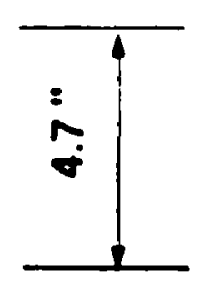

$0.895 \mathrm{lb} / \mathrm{in}$
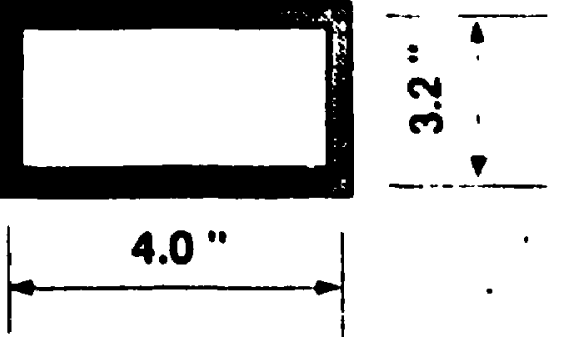

Section 2-2

Fig. 6.22 Experimental model by Kristek to check second of his analysis method.

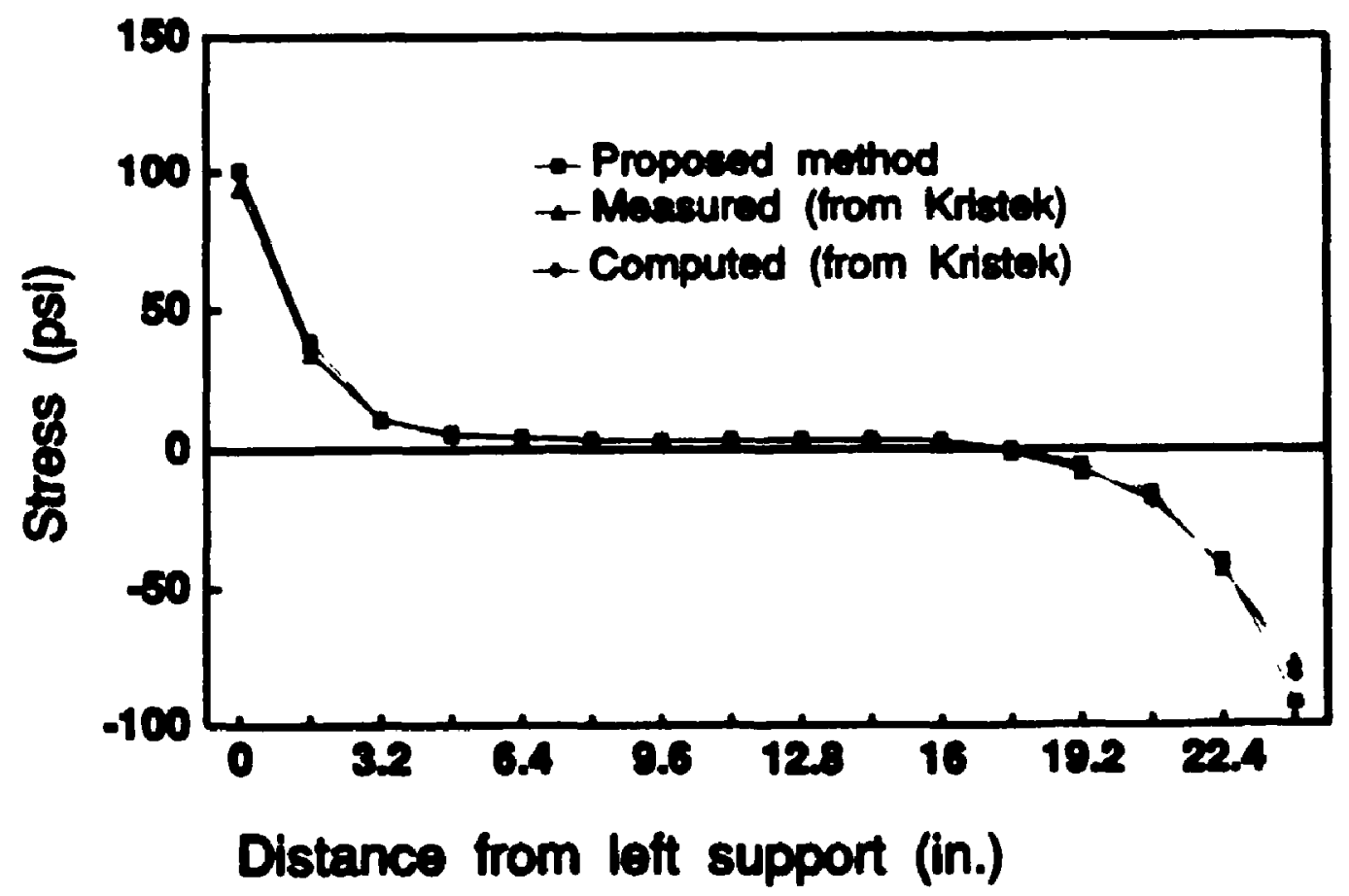

Fig. 6.23 Comparison of Longitudinal warping stresses along span. 


\begin{tabular}{|c|c|c|c|c|c|}
\hline $\begin{array}{l}\text { Distance from } \\
\text { support, in } \\
\text { inches }\end{array}$ & $\begin{array}{l}\text { Present } \\
\text { Method" }\end{array}$ & $\begin{array}{l}\text { Measured } \\
\text { deflections } \\
\text { from Kristek }\end{array}$ & $\begin{array}{l}\text { Computed } \\
\text { deflectlons } \\
\text { from Kristek }\end{array}$ & $\begin{array}{l}\text { Computed } \\
\text { deflections } \\
\text { from Mikkola }\end{array}$ & $\begin{array}{l}\text { Computed } \\
\text { deflections } \\
\text { from Bazant a }\end{array}$ \\
\hline $\begin{array}{l}0 \\
4 \\
8 \\
12 \\
16 \\
20 \\
24\end{array}$ & $\begin{array}{l}0 \\
0.0767 \\
0.3397 \\
0.5501 \\
0.7391 \\
0.8622 \\
0.9082\end{array}$ & $\begin{array}{l}0 \\
0.075 \\
0.351 \\
0.555 \\
0.741 \\
0.859 \\
0.907\end{array}$ & $\begin{array}{l}0 \\
0.068 \\
0.332 \\
0.541 \\
0.739 \\
0.869 \\
0.916\end{array}$ & $\begin{array}{l}0 \\
0.0734 \\
0.3476 \\
0.5511 \\
0.7402 \\
0.8600 \\
0.9073\end{array}$ & $\begin{array}{l}0 \\
0.074 \\
0.348 \\
0.550 \\
0.740 \\
0.859 \\
0.907\end{array}$ \\
\hline \multicolumn{6}{|c|}{$\begin{array}{l}\text { Deflections are in inches } \times 10^{-2} \\
\text { Note : } 1 \mathrm{in} .=25.4 \mathrm{~mm} .\end{array}$} \\
\hline
\end{tabular}

Table 6.1 Deflections of nodal edges of tapered box girder.

Distance from left support (in.)

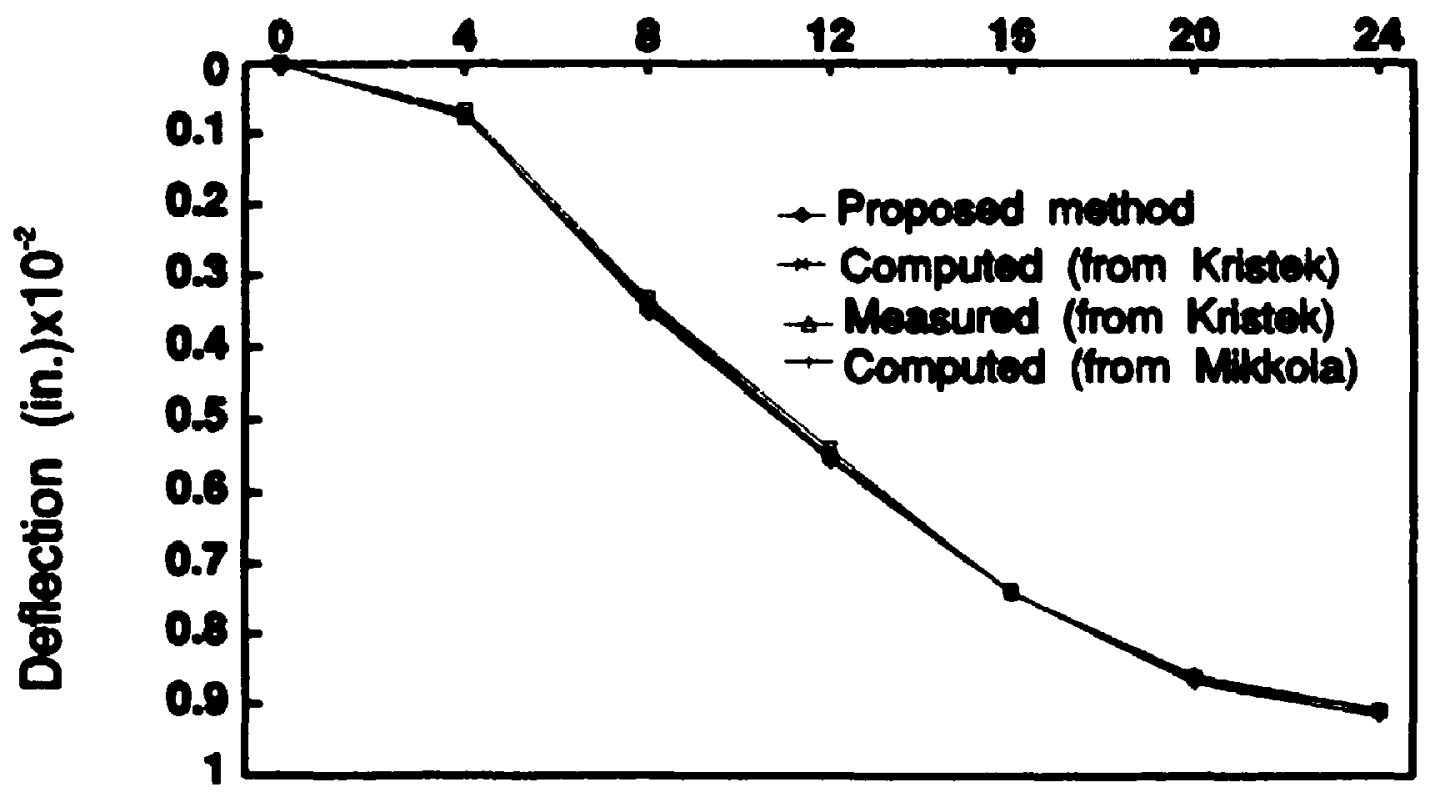

Fig. 6.24 Deflection curved in second step of Kristek's analysis. 


\subsubsection{Two-Cell Skewed Box Girder Bridge}

A simply supported two-cell skewed box girder bridge from Scordelis (1971) has been analysed by the proposed method. The skew angle of the structure is $45^{\circ}$ and the dimensions are shown in Figs. 6.25(a) and 6.25(b). The modulus of elasticity is $432000 \mathrm{kip} / \mathrm{ft}^{2}$, and the Poisson's ratio is 0.15 . The girder is subjected to a single point load of 1 kip placed over the web at midspan. Based on the Ontario Highway Bridge Design Code (1983), the skew angle for such a bridge can not be neglected and has to be considered in the analysis.

Twenty thin-walled box beam elements are used to model the whole structure. Fig. 6.25(c) illustrates the distribution of longitudinal stress at the skewed midspan section A-A. The results are compared with those from finite element method given by Scordelis (1971), and the agreement between the two results is good, with the maximum differences in this case being $14 \%$. 


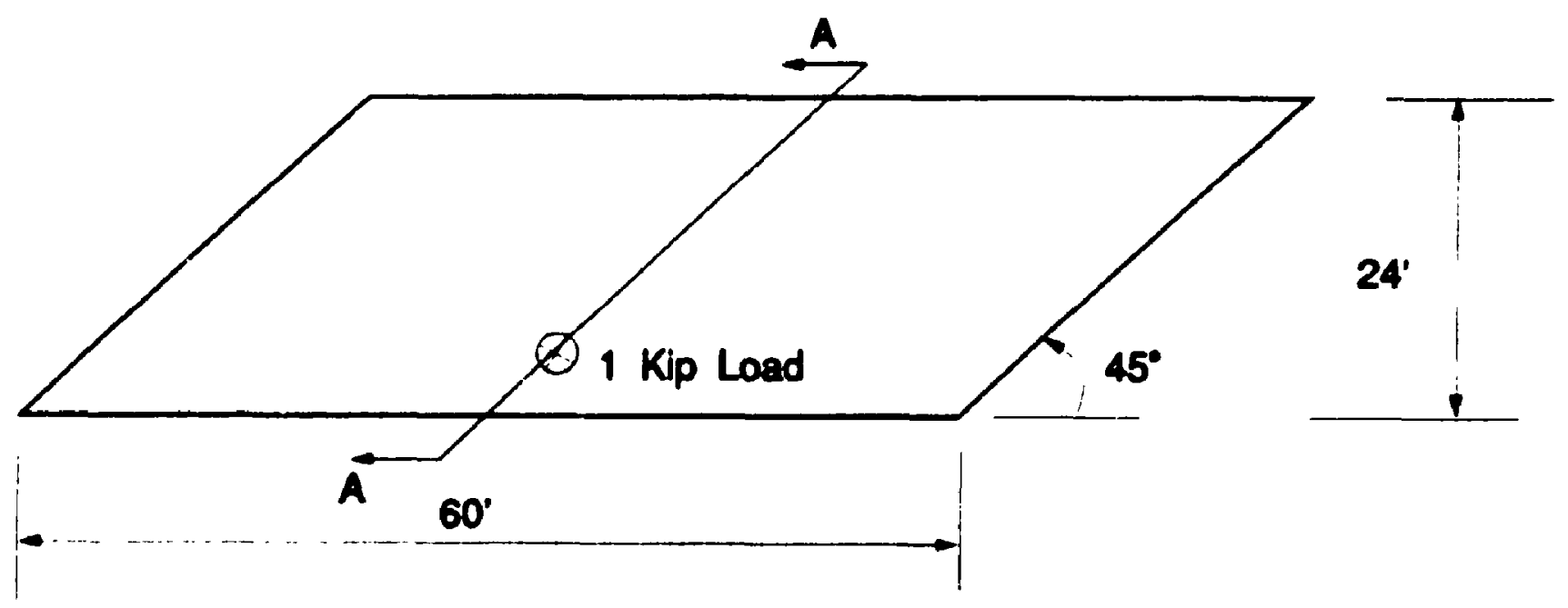

(a) Plane.

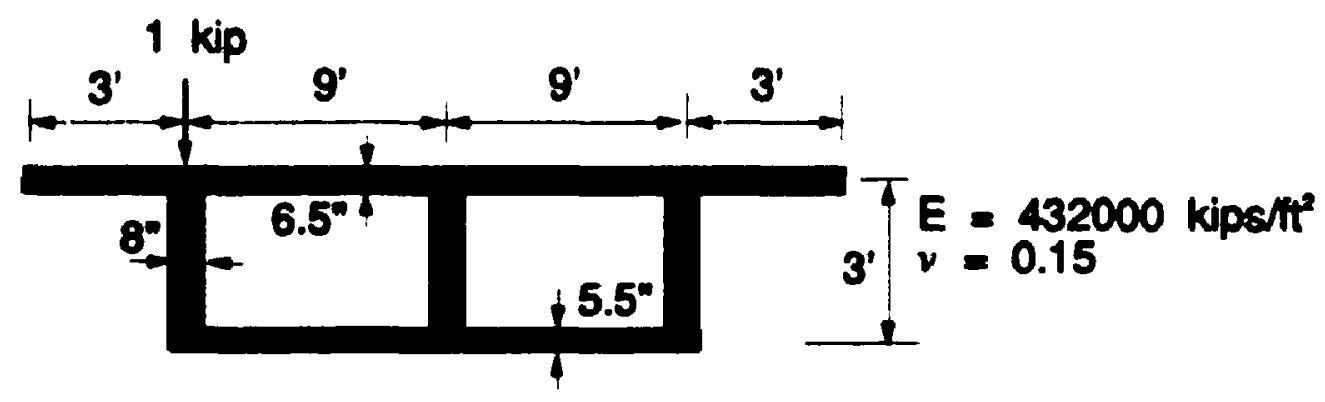

(b) Cross section A-A.

-0.769 Proposed method

$(-0.732)$ Finte element method from Scordelis (1971)

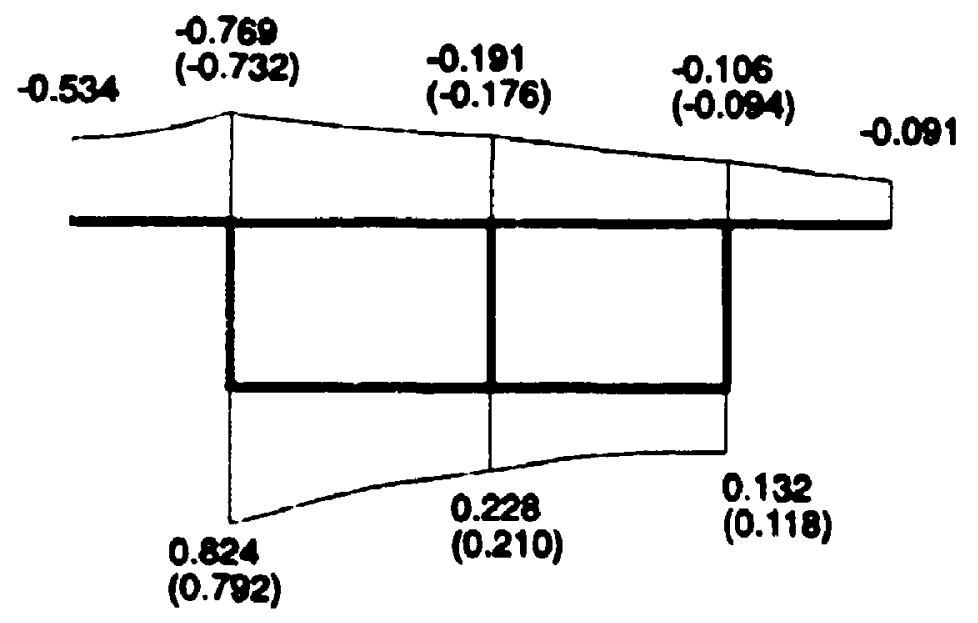

(c) Longitudinal stress distribution in cross section A-A.

Fig. 6.25 Two-cell skewed box girder bridge. 


\subsubsection{Single-Cell Curved Box Girder Bridge}

A single-cell curved box girder bridge model from Moffat and Lim (197i), as shown in Fig. 6.26, is taken as another example. Jirousek and Bouberguig (1979) also analyzed this problem by their macro thick shell finite elements.

The dimensions of the curved box girder are shown in Fig. 6.26. The modulus of elasticity is $2750000 \mathrm{psi}$, and the Poisson's ratio is $\mathbf{0 . 2 3}$. The girder is subjected to a single point load of $100 \mathrm{lb}$ placed over the outer web at midspan.

Because of symmetry, the present analysis was carried out for only half of the structure with eight thin-walled curved box beam elements. The vertical deflections and the longitudinal normal stresses at midspan section are plot ted in Figs. 6.27(a) to 6.28(b), respectively, and compared with those obtained by Jirousek and Bouberguig (1979). It can be seen the results generated by the present element are fairly close to the thick shell element results, and the maximum difference doxs not exceed $10 \%$.

\subsubsection{Two-Cell Curved Box Girder}

A two-cell curved box girder bridge is analyzed to iilustrate the versatility of the present theory for describing the structural behaviour of multicell curved box girder structures. The curved box girder was initially presented by Meyer (1970) to illustrate the validity of the curved finite strip theory and of the computer program CURSTR. By means of the program CURSTR it is possible to analyse prismatic folded plate structures curved in plane and simply supported along their straight radial edges. $\mathrm{Li}$. Tham and Cheung (1988) examined the same bridge by spline finite strip method.

The structure ha, adius of $76.2 \mathrm{~m}$ and a deflection nugle 0.4 radian. The bridge deck is of width $8.534 \mathrm{~m}$ with overhangs of $0.914 \mathrm{~m}$. The coutrer and inuer webs are inclined. and the middle web is vertical. The flange and webs are of thickuess $0.165 \mathrm{~m}$ and $0.203 \mathrm{~m}$, respectively. The bridge is loaded by $\mathrm{s}$ six-wher truck in the outer lane. The position of each wheel and its loading are shown in Figs. 6.28(a) and $6.28(b)$. 
Nineteen thin-walled curved box beam elements are used for the whole bridge. The midspan longitudinal normal stress, Fig. 6.29(a), and the transverse bending stress, Fig. 6.29 (b), are compared with the results given by $\mathrm{Li}$, Tham and Cheung (1988), and as can be seen they are in good agreement. The maximum difference in this case is $11 \%$.

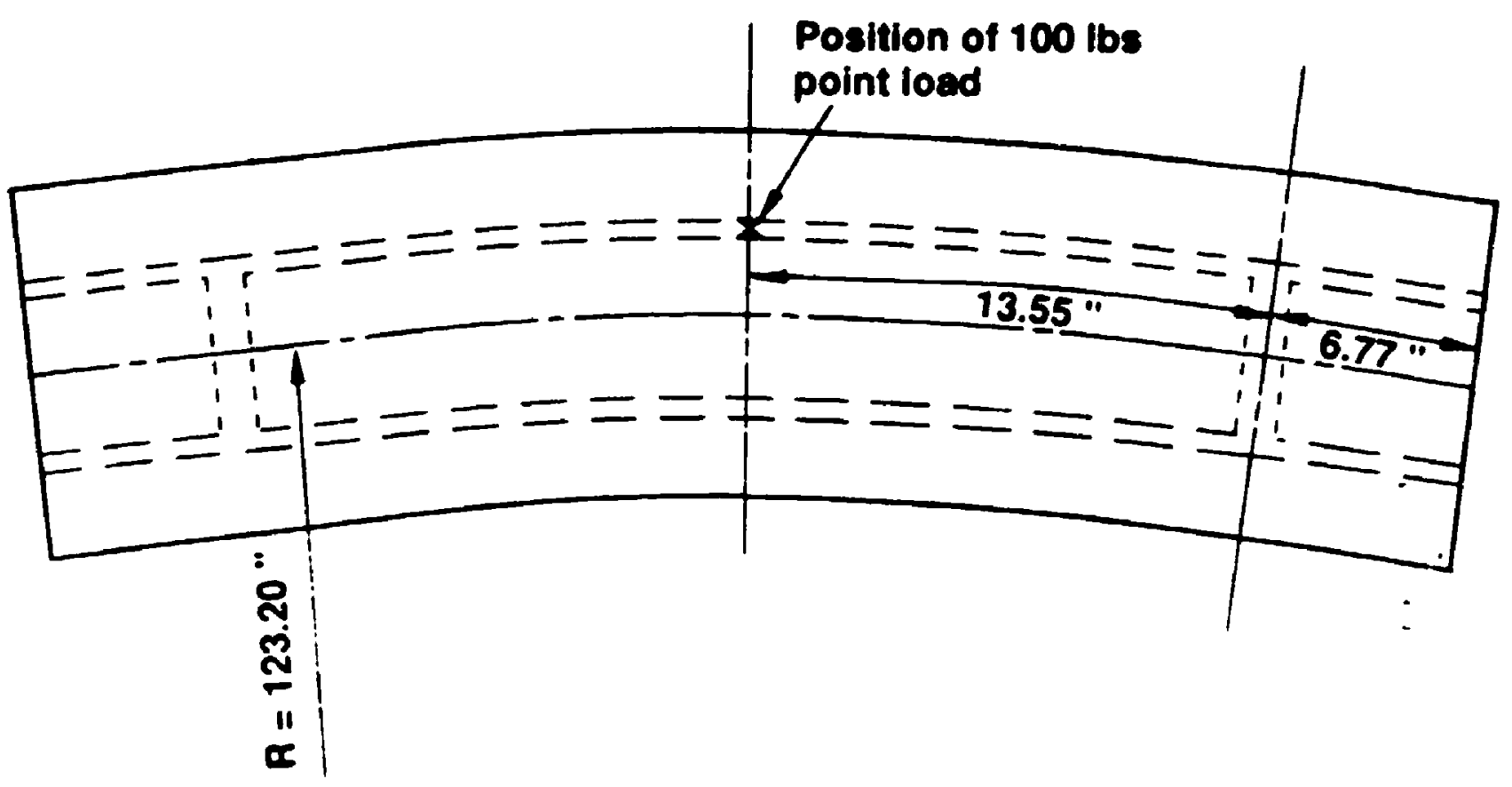

(a) Plan View

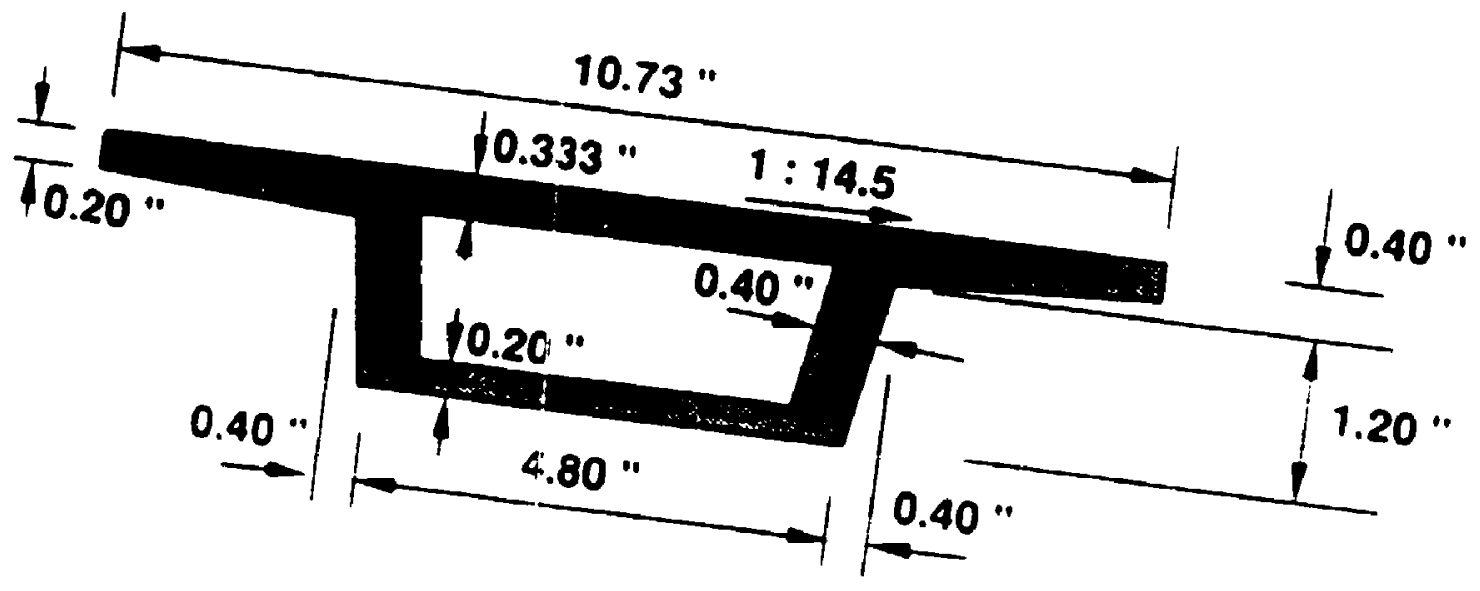

(b) Cross-Section

Fig. 6.26 Single-cell curved box girder. 
() Jirousek and Bouberguig (1979)

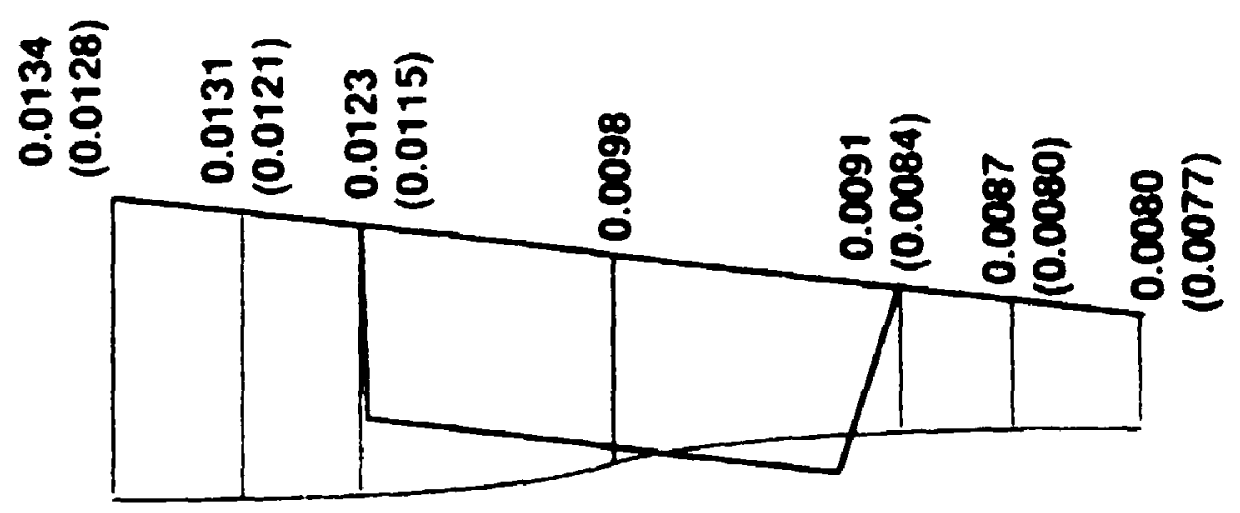

(a) Deflection of top flange at midspan (in.).

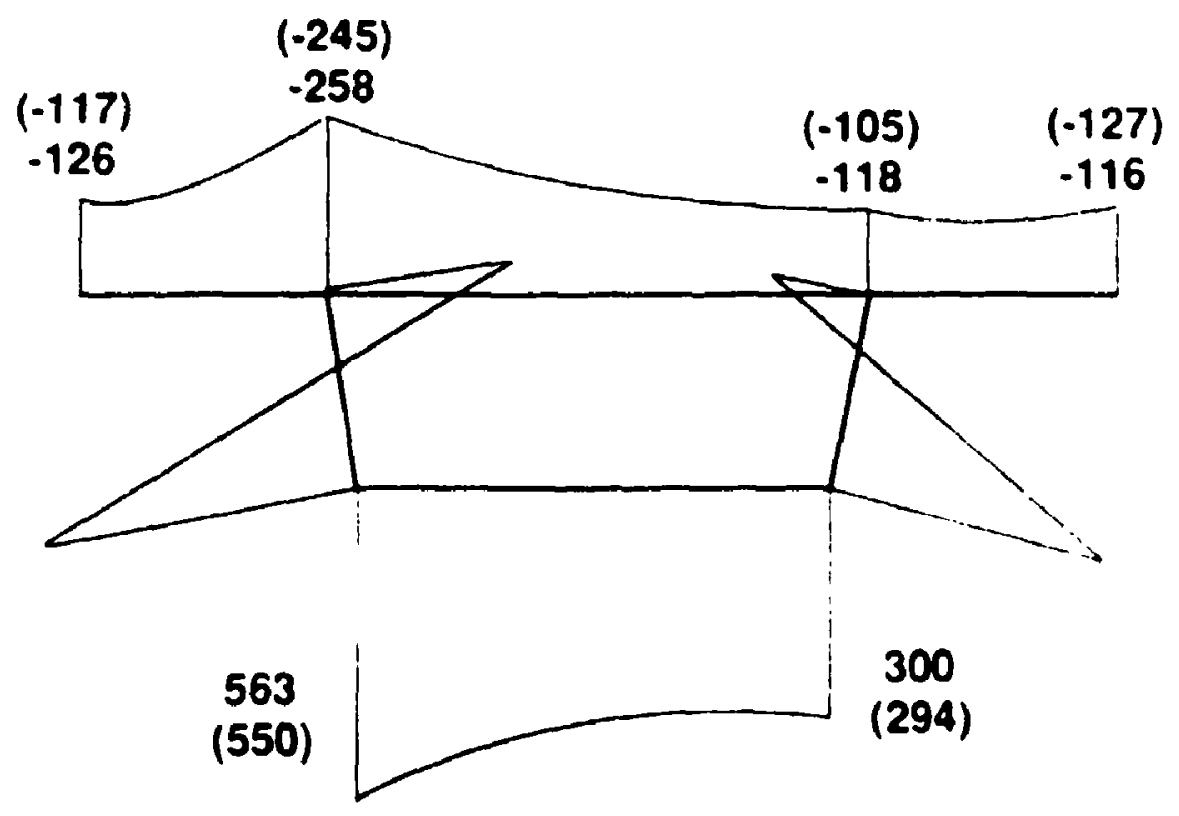

(b) Longitudinal normal stress at midspan (psi).

Fig. 6.27 Comparison with macro thick shell finite element method. 


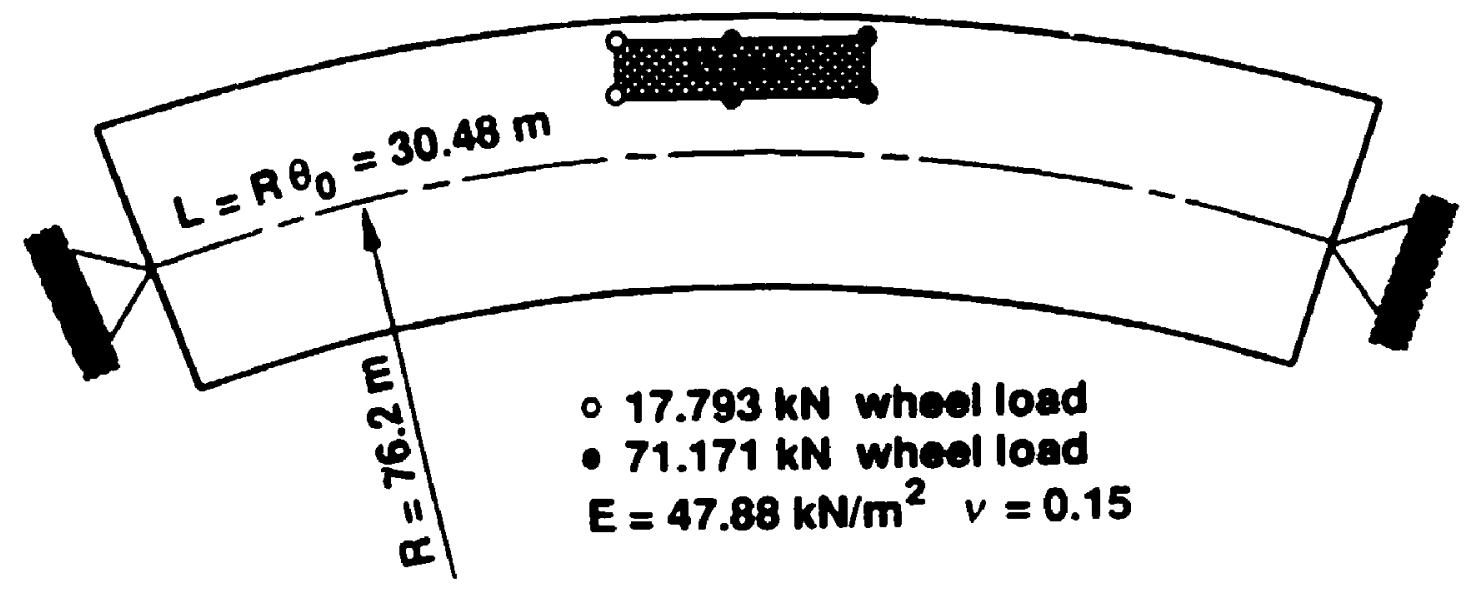

(a) Plan View

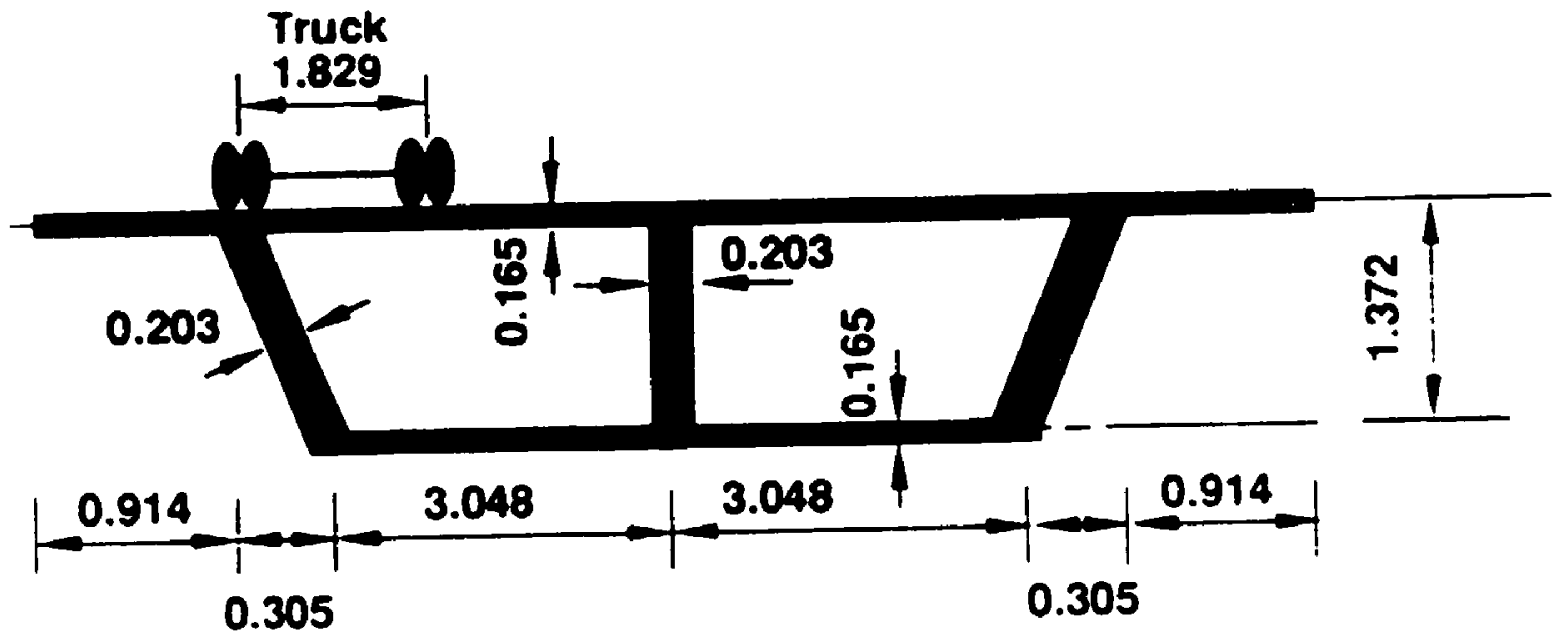

(b) Cross-Section

Fig. 6.28 Two-cell curved box girder. 
() Li et. al (1988)

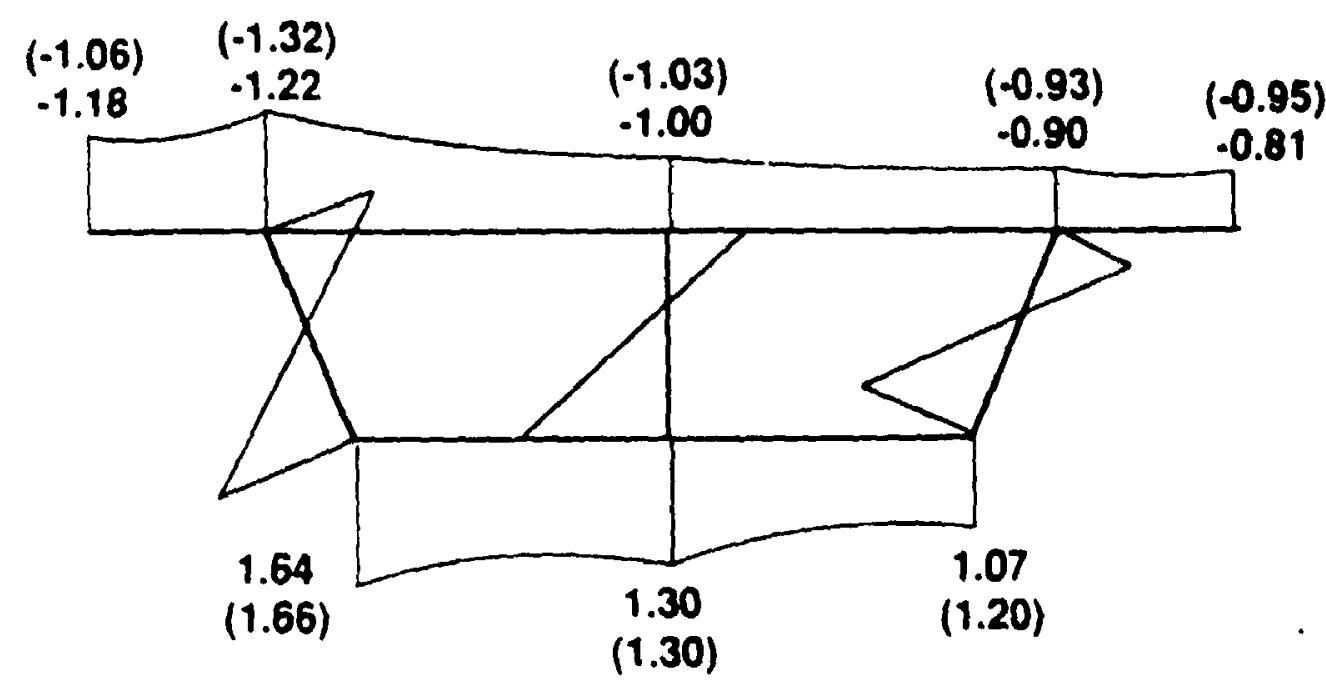

(a) Longitudinal normal stress at midspan (MPa).

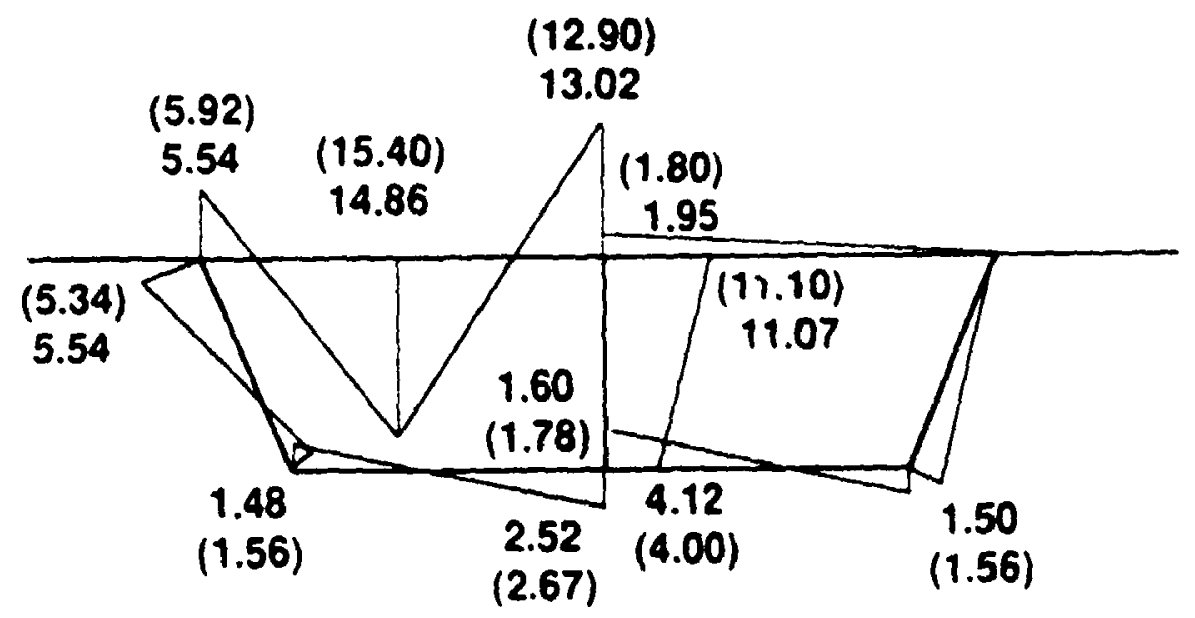

(b) Transverse bending moment at midspan $(\mathrm{k} N-\mathrm{m} / \mathrm{m})$. Fig. 6.29 Comparison with spline finite strip method. 


\subsubsection{Multi-Branch Box Girder Assemblage}

As a final example for this section, a multi-branch concrete box girder, subjected to the Ontario Highway Bridge Design truck (OHBDC 1983) on branch 1 and 3 over the webs, Fig. 6.30 , is analyzed. The box girder has diaphragms only at the supports. The diaphragms endow the box with full torsional and distortional resistance, but with no resistance to warping. The dimensions, loading and material properties of the cross section are shown in Fig. $\mathbf{6 . 3 0}$.

A rigid arm is used to model the stiff behaviour of the intersection region. For branches 1 and 2, only one straight exact shape function thin-walled box beam element developed in Chap. 3 is used. For branch 3, fifteen curved thin-walled box beam elements developed in Chap. 4 are used to model this curved branch. Facet shell finite analysis is also carried out for the sake of comparsion. A total of 268 shell finite elements are used in the analysis.

Figures 6.31 and 6.32 show the variation along the span of the longitudinal normal stress at junction $A$, obtained from various beam theories. Figures 6.33(a) and 6.33(b) show the longitudinal normal stress at midspan for branches 1 and 3 due to truck loads only. The results are compared with facet finite element analysis and engineer's beam theory. It can be seen that the proposed model results are in good agreement with those of shell finite element, except near the junction of the three members which is not unexpected because the basic theory upon which the present formulation is based can not account for such local effects.

It is important to mention that the proposed procedure allows the designer to assess the contribution of each type of structural action separately and to determine its relative significance. Furthermore, the computer time needed to analyze complex box girder bridges by the proposed method does not exceed a few seconds even on a microcomputer. The computer memory requirements and input data are minimal und thus the method is ideally suited to microcomputers. 

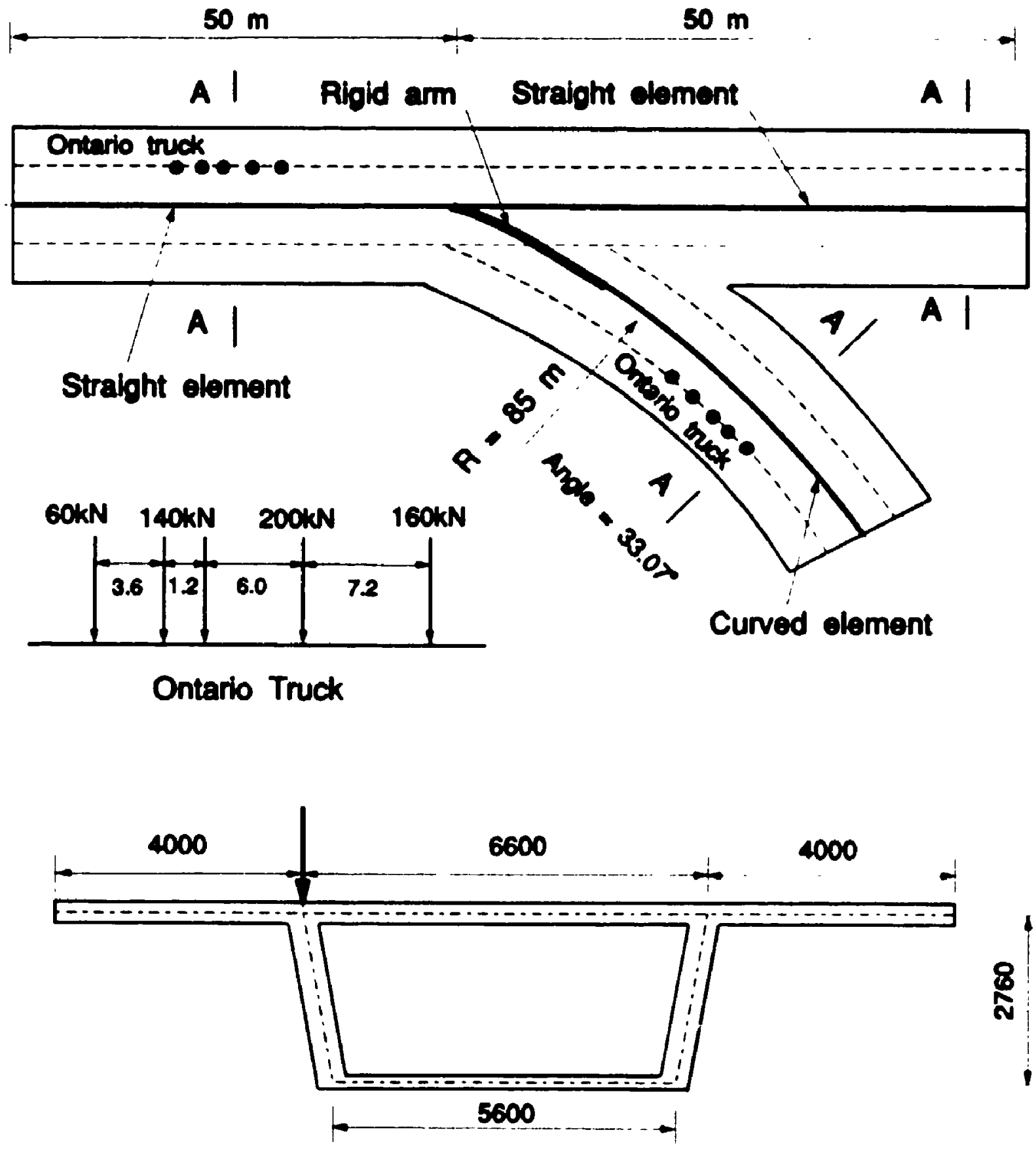

Fig. 6.30 Multi-branch box girder assemblage. 


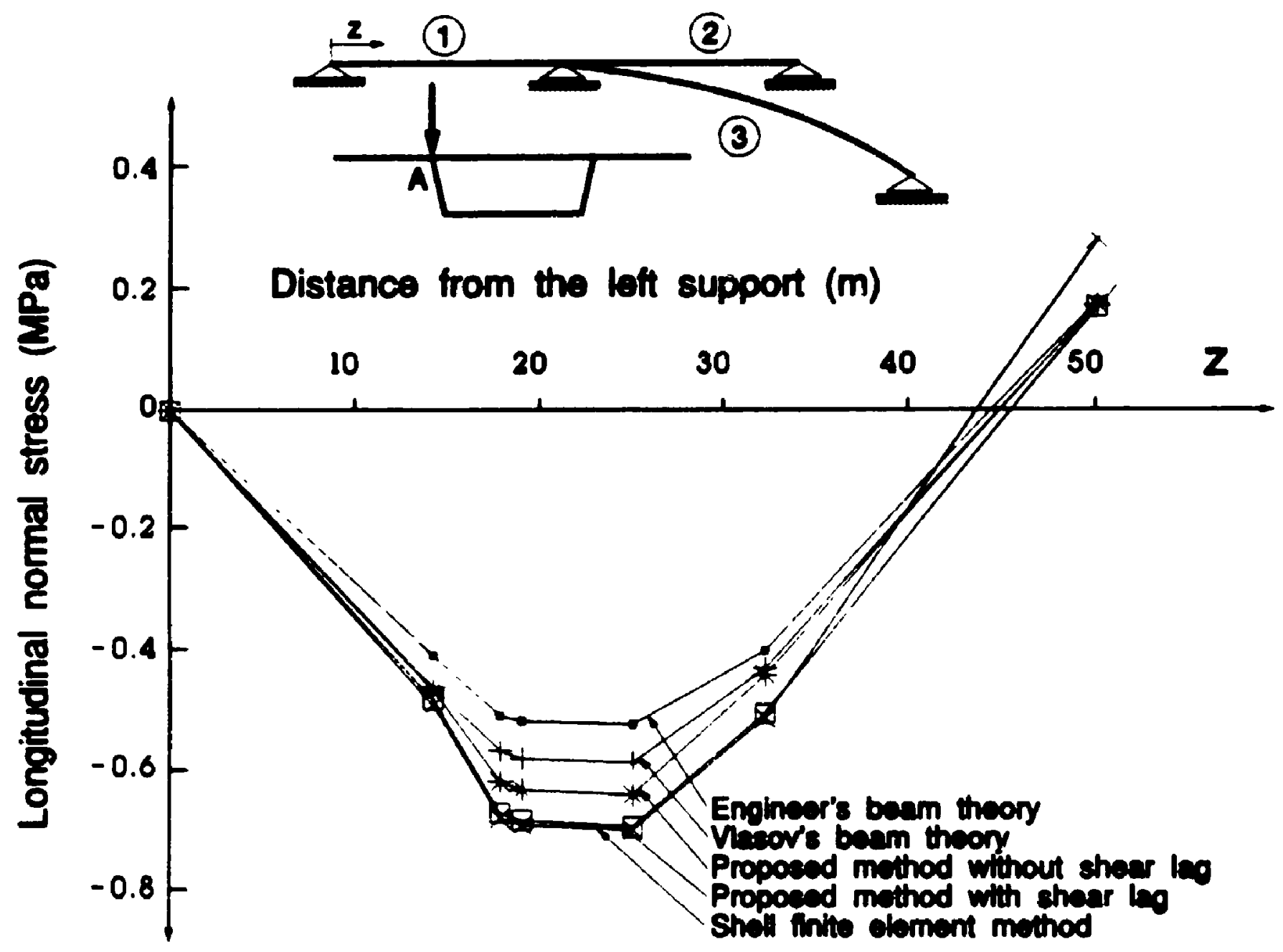

Fig. 6.31 Variation of longitudinal stress along junction $A$ for branch 1 (MPa). 
(1) (2)

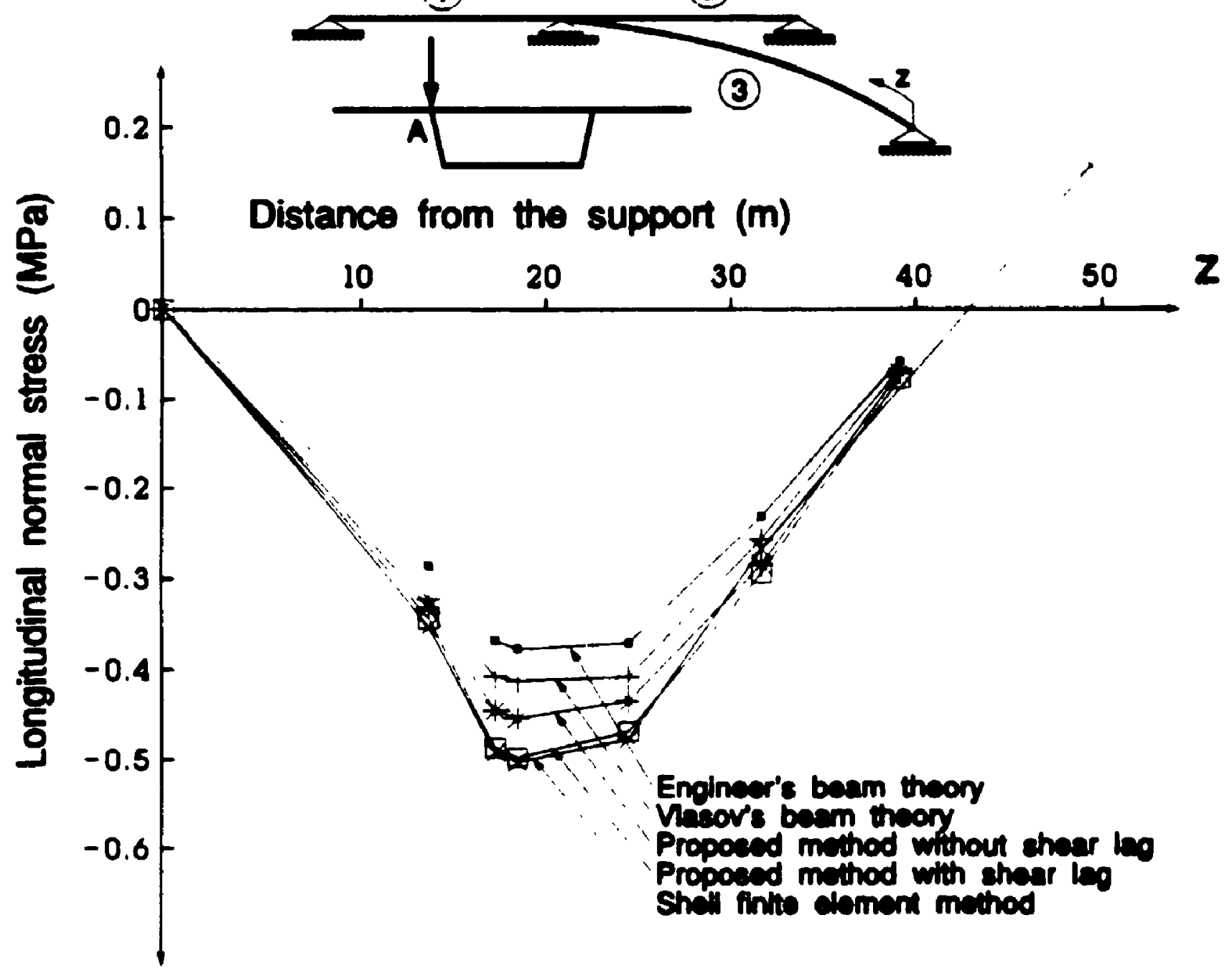

Fig. 6.32 Variation of longitudinal stress along junction $A$ for branch 3 (MPa). 
1-0.000] Brall the clament

-0.000 Propoed method

$(-0.500)$ Engineor's bean theory

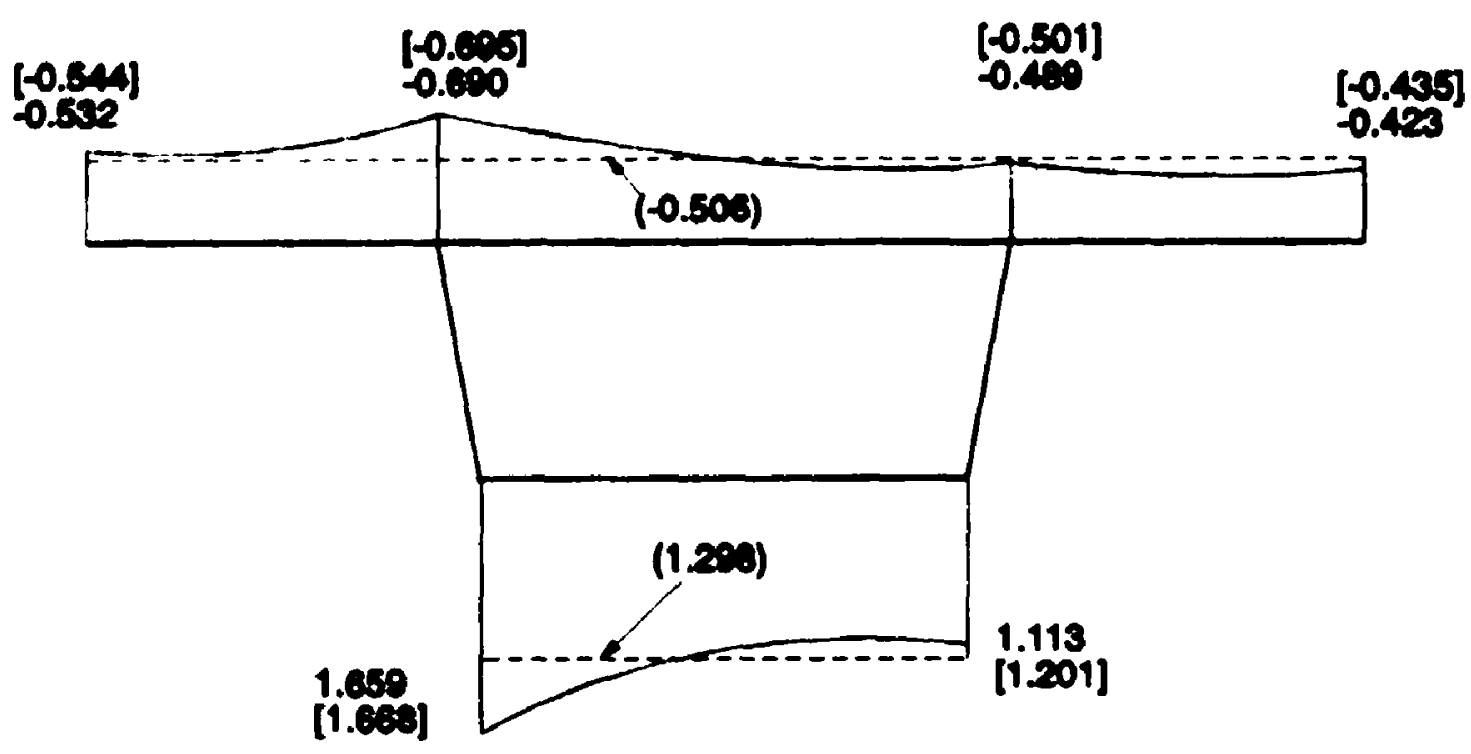

(a) Midepan at branch 1

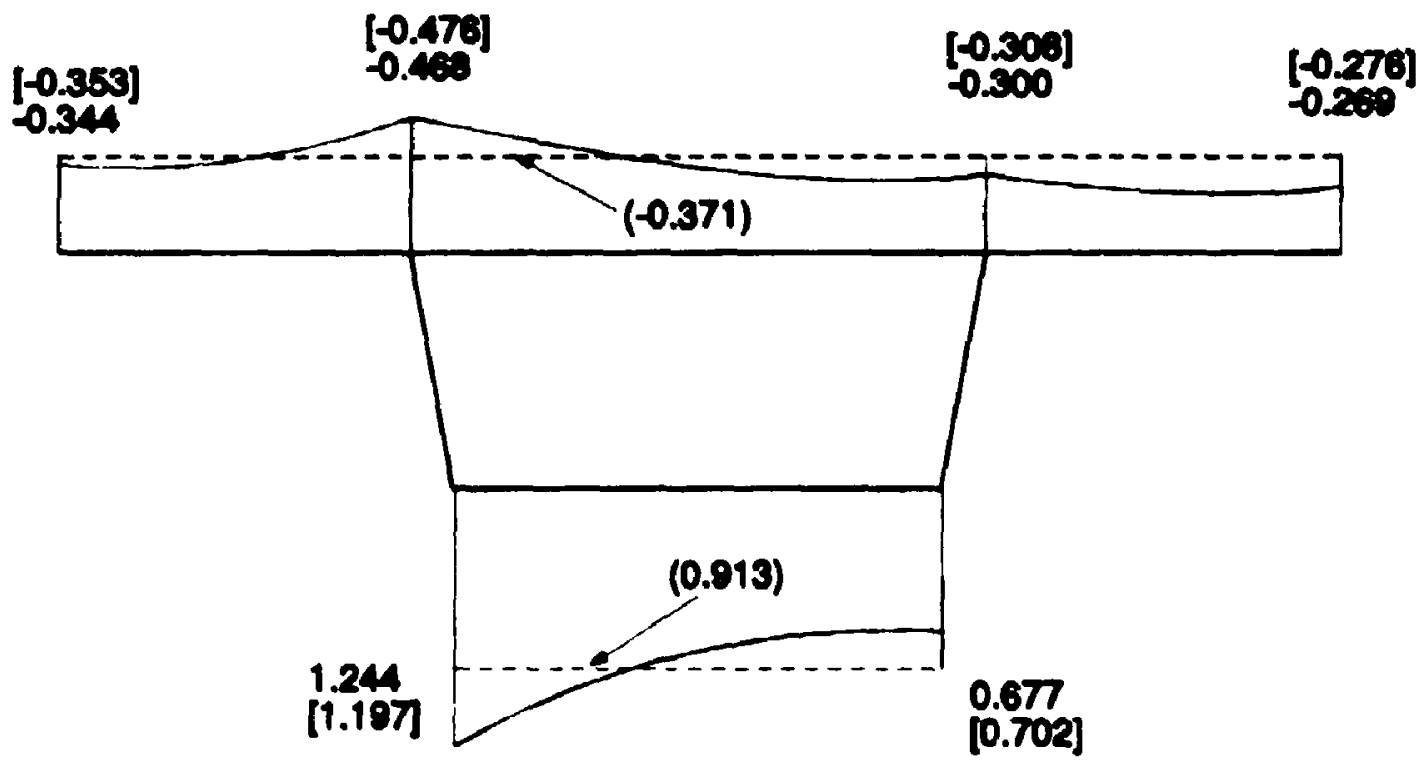

(b) Midspan at branch 3

Fig. 6.33 Longitudinal normal stress along cross sections ( $\mathrm{MPa})$. 


\subsection{Prestressing, Temperature, Creep and Shrinkage Effects}

'io demonstrate the applicability of the methods implemented in Chap. 5 of the present study and the corresponding computer program CBOX to the analysis of prestressing, temperature and time dependent effects of box beams, five box girder structures are selected for analysis. In the first example, a single-cell curved concrete box girder subjected to prestressing force is analyzed. For temperature stresi analysis, one concrete box girder and one composite box girder are analyzed for the comparison of temperature stresses in those two common forms of bridges. In thr fourth example a two-cell two span composite box girder is examined for shrinkage effect of the concrete, while the fifth example concerns the creep analysis of a two-cell two span box girder structure. The results are compared with those obtained from facet shell finite element finite ard analytical methods.

\subsubsection{Prestressing Box Girder Bridge}

To show the possibility of adequately representing the effect of prestressing, a curved box girder bridge of Fig. 6.34 is studied using the concept of equivalent primary nodal forces presented in Section 5.2. Each web of the bridge is provided with one parabolic cable. The prestressing forces are designed to balance the selfweight of the structure. The intensity of the cable tension is assumed constant. The dimensions of the structure and the cable profile are shown in in Fig. 6.34.

Owing to symmetry, the analysis was performed considering only one half of the bridge. Ten thin-walled beam elements are used in the analysis. The equivalent lond method which transform the prestressing cable forces into equivalent statir londs are also carried out. The results are compared with those obtained from shell finite element method. Due to symmetry half the box girder was used in the annlysis and 144 quadrilateral elements were employed.

Stresses resulting from the effect of prestressing bending are shown in Fig. 6.35, 
and prestressing camber is shown in Fig. 6.36. As can be noticed all three sets of results are in close agreement, with a maximum deviation of $9 \%$ between the shell finite element and the proposed method. It can be seen that even under symmetric loading conditions for curved box girder, the structure is still under torsional and distortional actions. It is therefore concluded that the equivalent load method can be used for practical purpose with confidence.
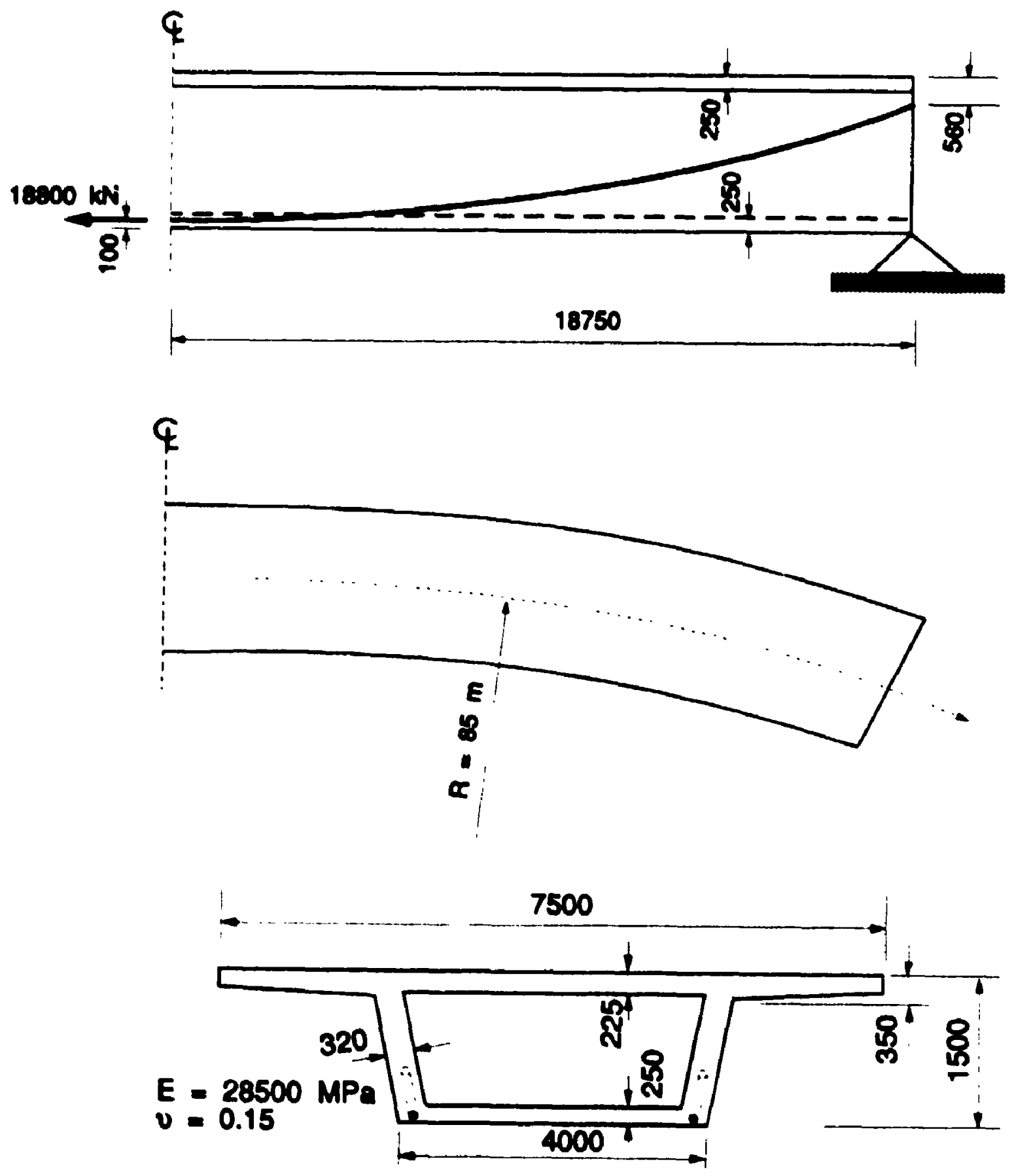

Fig. 6.34 Prestressed box girder ( $\mathrm{mm}$ ). 
1.77 Proposed mothod (using program CBOX directly) [1.63] Shell finite olement

(1.70) Equivalent load (using program CBOX)

\begin{tabular}{|c|c|c|}
\hline $\begin{array}{l}1.42 \\
\left\{\begin{array}{l}1.50 \\
1.48\end{array}\right)\end{array}$ & $\begin{array}{l}1.55 \\
\left\{\begin{array}{l}1.52 \\
1.50\end{array}\right.\end{array}$ & $\begin{array}{l}1.77 \\
{\left[\begin{array}{l}1.63 \\
1.70\end{array}\right.}\end{array}$ \\
\hline
\end{tabular}

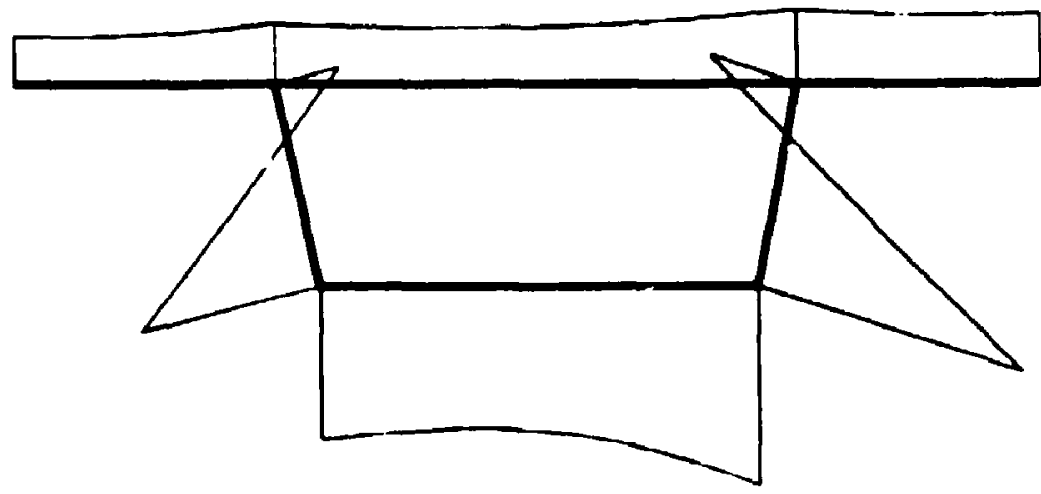

$-15.79$

$[-15.07$

$\{-15.94)$
$-17.89$

$[-16.73]$

Fig. 6.35 Longitudinal stress at midspan due to prestressed force (MPa).

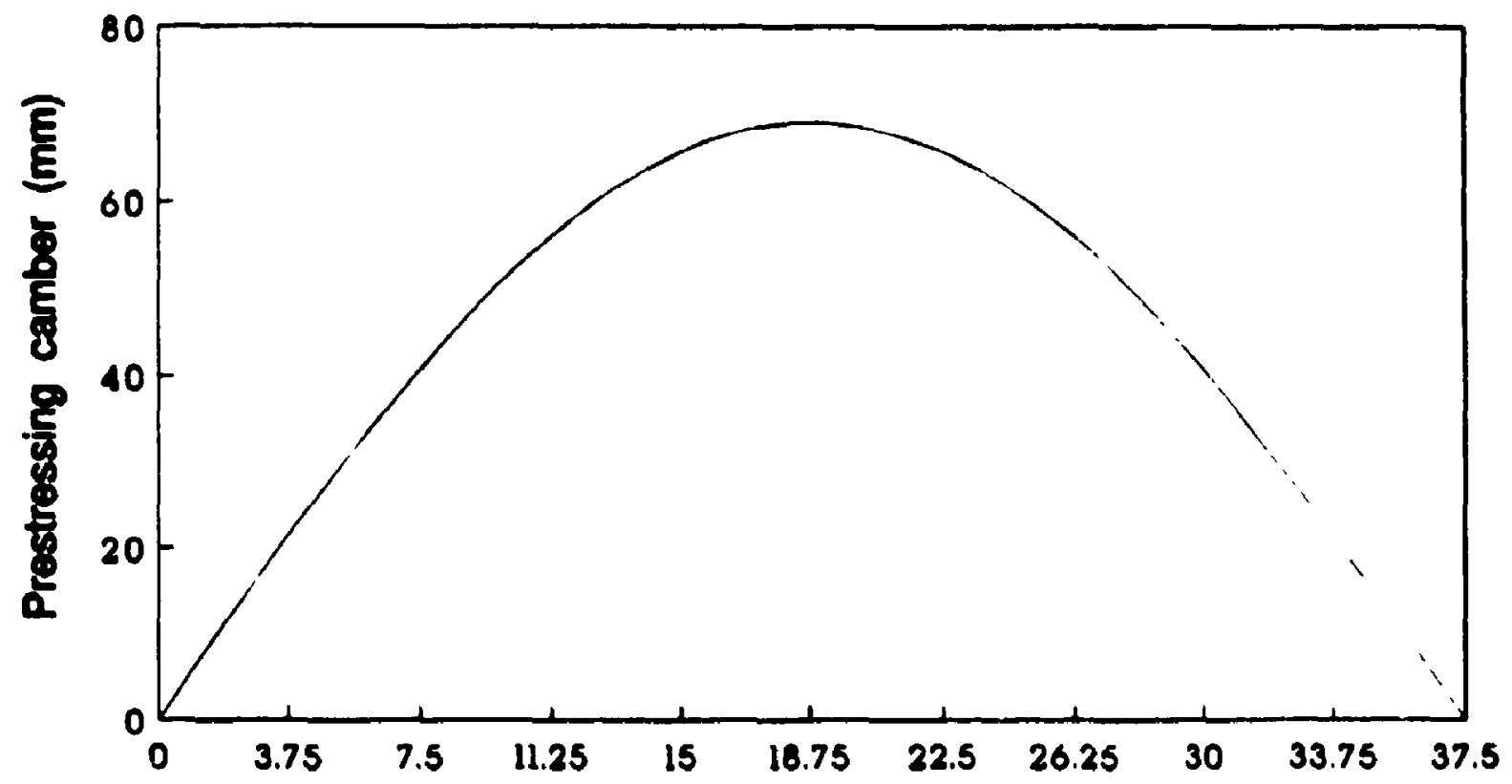

Distance from left support $(m)$

Fig. 6.36 Prestressing camber $(\mathrm{mm})$. 


\subsubsection{Thermal Stress Analysis for Box Girder}

A concrete box girder from Moore (1975) and a composite box girder are selected for the comparison of temperature stresses in those two most common used structures. Both structures have the same dimensions and are single span and simply supported box girders. The cross sections of these structures are shown in Fig. 37. Priestley's (1976) six order temperature distributions described in Sec. 5.3.1 are used in the analysis. The thickness of asphalt pavement is assumed to be $90 \mathrm{~mm}$ which is common practice in Ontario. The temperature distributions are re-plotted in Fig. 6.38.

Figs. 6.39(a) and 6.39(b) show the longitudinal thermal stresses due the temperature distributions for concrete anc composite box girders, respectively. Good comparsion between the proposed method and the shell finite element method given by Moore (1975), and the analytical solutions calculated by hand (Ghali and Favre 1986). The maxmium difference between the proposed method and shell finite element inethod in concrete box girder is $11 \%$, while in composite the difference between the proposed method and the analytical method in the composite box girder it is $3 \%$. It can be seen that some tensile stresses develop in the concrete deck in the composite box girder, caused by the nonlinear temperature distribution. We may also notice that the thermal stresses in the composite box girder are much ingher than thone in the concrete box girder. 


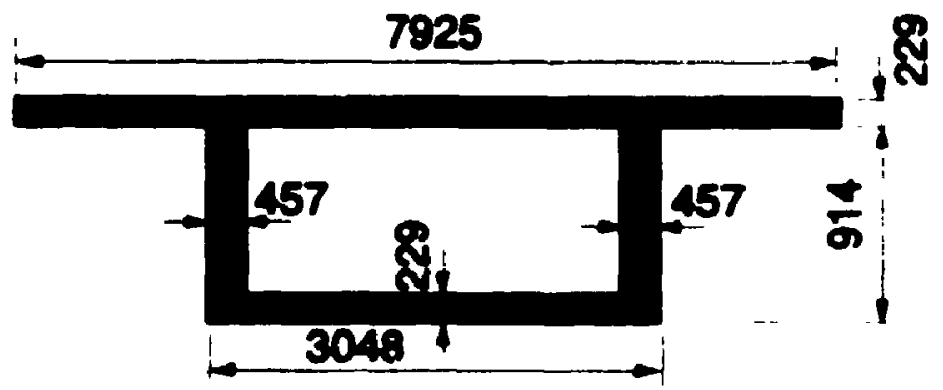

E. $35000 \mathrm{MPa}$

$v=0.15$

$\alpha=10.8 \times 10^{\circ} \% \mathrm{C}$

(a) Concrete box girder.

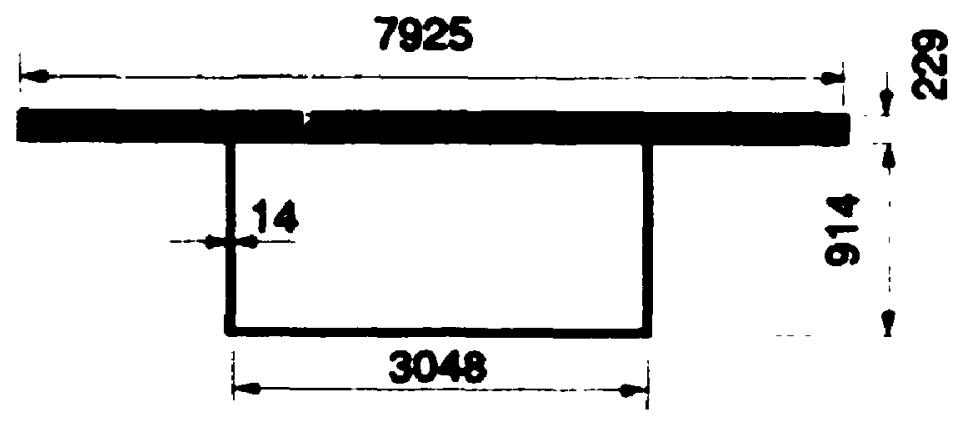

$E_{0}=35000 \mathrm{MPa}$

$E_{1}=200000 \mathrm{MPa}$

$\alpha=10.8 \times 10^{\circ}$

$a_{1}=12 \times 10^{\circ}$

(b) Composite box girder.

Fig. 6.37 Box girders for thermal stress analysis.

$33.3^{\circ} \mathrm{C}$

$$
\begin{aligned}
& T(y) \\
& T(y)=33.3\left(\frac{y}{D}\right)^{\circ}
\end{aligned}
$$

Fig. 6.38 Temperature distribution. 
-4.76 From proposecl method by program CBOX

[-4.82) From shell fintio element by Moore (1975)

(-4.63) From andyical mothod given by chali and Favre (1986)

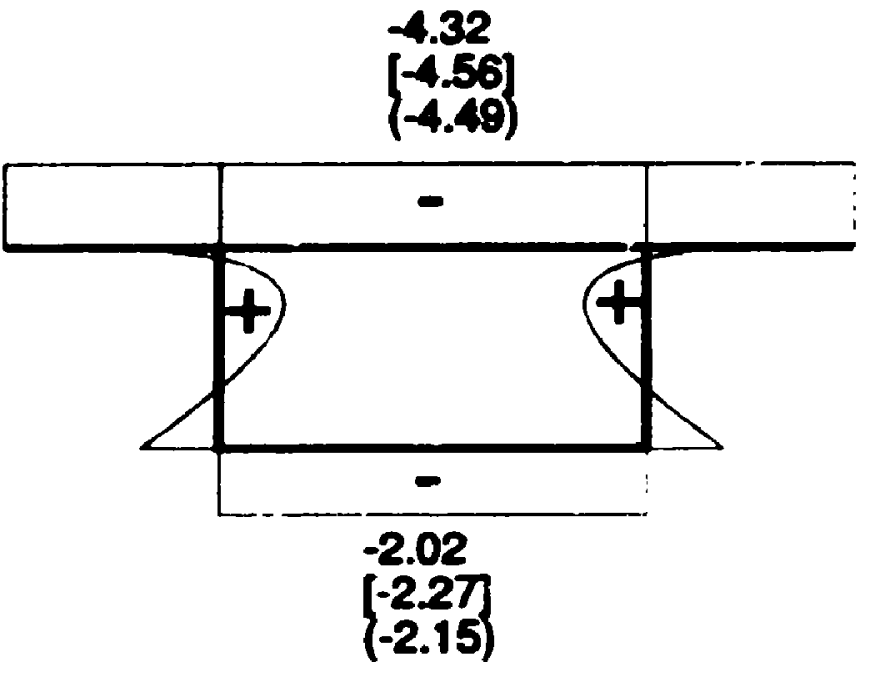

(a) Longitudinal thermal stress for concrete box girder.

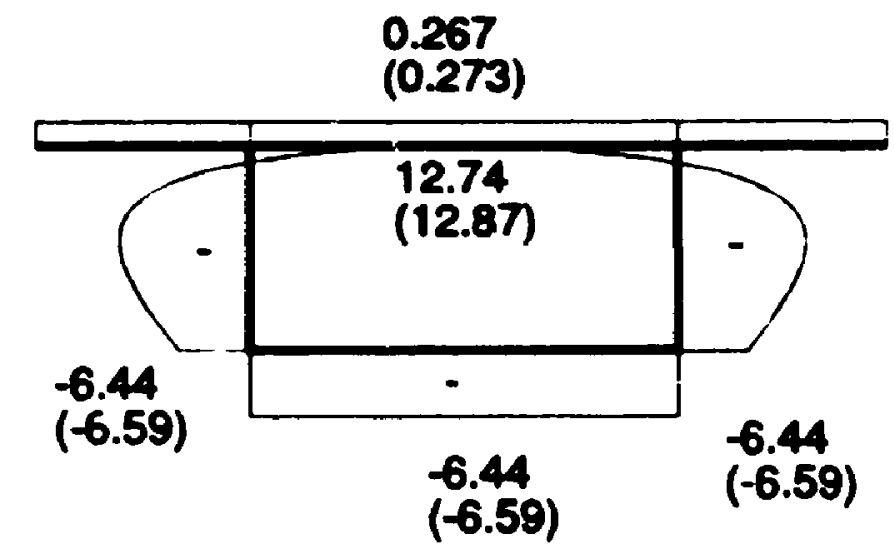

(b) T.ongitudinal thermal stress for composite box girder. F ag.39 Longitudinal thermal stress distribution (MPa). 


\subsubsection{Shrinkage Analysis of Composite Box Girder Bridge}

Figure 6.40 shows a two-cell two span continuous composite box girder. Mloist cured concrete under standard conditions is assumed for the concrete deck. At initial age $t_{0}=28$ days. the modulus of elasticity of concrete deck is $28500 \mathrm{MPa}$. and the Possion's ratio is 0.15 . The modulus of elasticity for steel is $200000 \mathrm{MPa}$ and the Possion's ration is 0.3. After $\left(t-t_{0}\right)=100$ days, from Eq. (5.4.8) the free shrinkage $\epsilon_{c s}=-310 \times 10^{-6}$.

Because the box girder is a statically inderminated structure, additional reactions are generated due to the non-uniform shrinkage of the structure as shown in Fig. 6.40. The longitudinal normal stress distrihutions at midspan and mid-support due to shrinkage are shown in Figs. 6.41(a) and 6.41(b). respectively. The results are compared with those obtained from the analytical method calculated by hund (Ghali and Favre 1986) The maximum difference at midspan bet ween the proposed unethod and analytical method is $6 \%$, while at mid-support is $12 \%$. Figure 6.41(b) shows some shear lag effect at mid-support section because the reaction due to non-uniform shrinkage of the structure can be consider as a concentrated load ucting at this section, and the analytical method can not predict these kinds of effects. It chn be seen that the whole cross section is under compressive stress due to the shrinkage of the concrete deck and the stress in the steel goes up to about $20 \mathrm{MPh}$.

\subsubsection{Creep Analysis for Concrete Box Girder Bridge}

The two-cell two span concrete box girder analyzed in Ser. 6.2 .2 is selecterd us the final example for creep analysis. The dimensions, loadings and material propertiess are shown in Fig. 6.6. The stresses at Sec. 6.2.2 are assumed to be the initial state at age $t_{0}=28$ days. Due to the sustained seif-weight, creep effect in the concrete will change the stress distribution gradually. After 10000 dhys $\left(t-t_{0}=10000\right.$ days $)$, the longitudinal stress distributions for midspan and mid-support are shown in Figh. 6.42(a) and 6.42(b), respectively. Comparing with Fig. 6.9, the longitudinal normal stress increases about $12 \%$ at midsupport due to long term creep of concrete. 

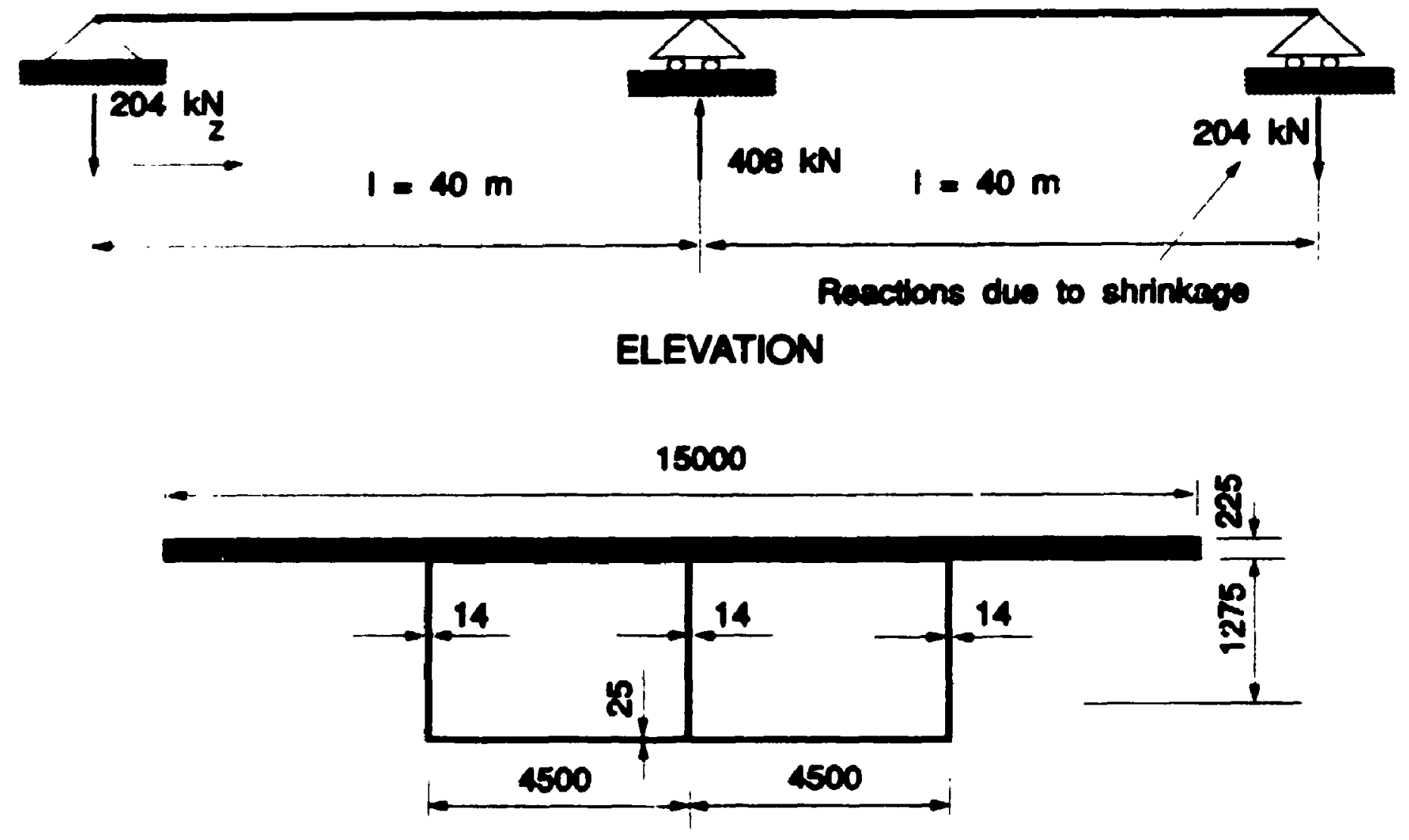

CROSS SECTION

Fig. 6.40 Two-cell two span composite box girder. 
2.24 From proposed method by program CBOX

(2.09) From analytical mothod by Chall and Favro (1886)

2.22

(2.09)

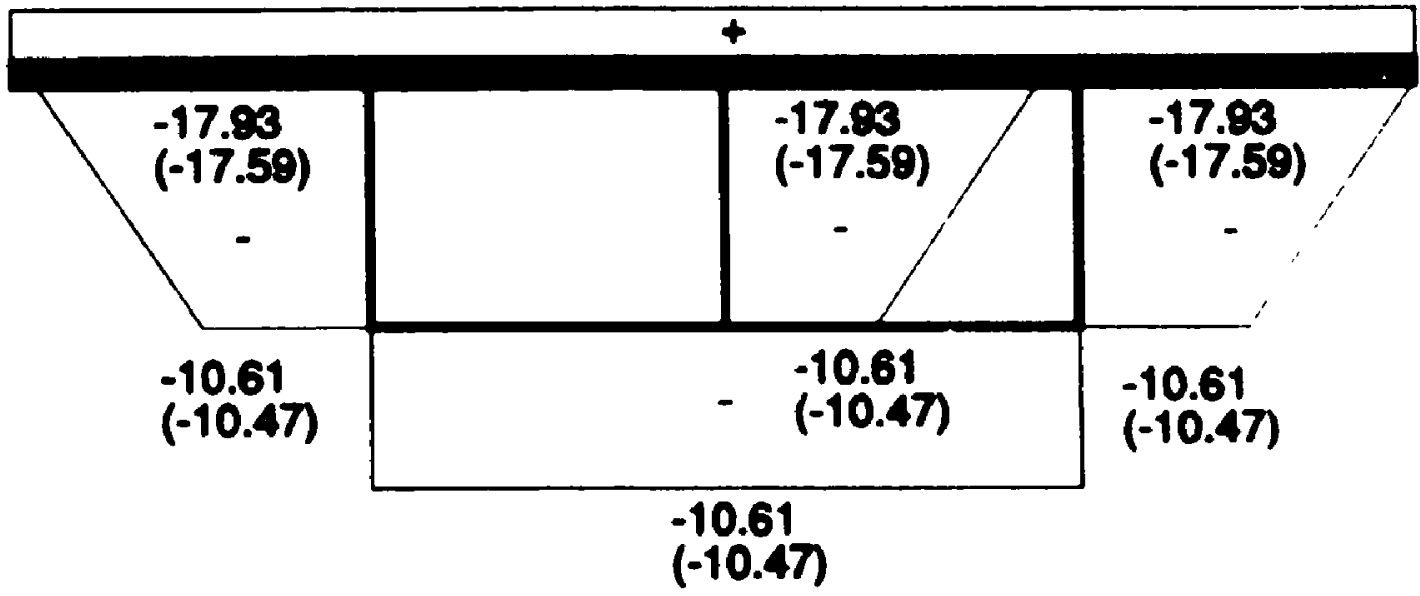

(a). At midspan.

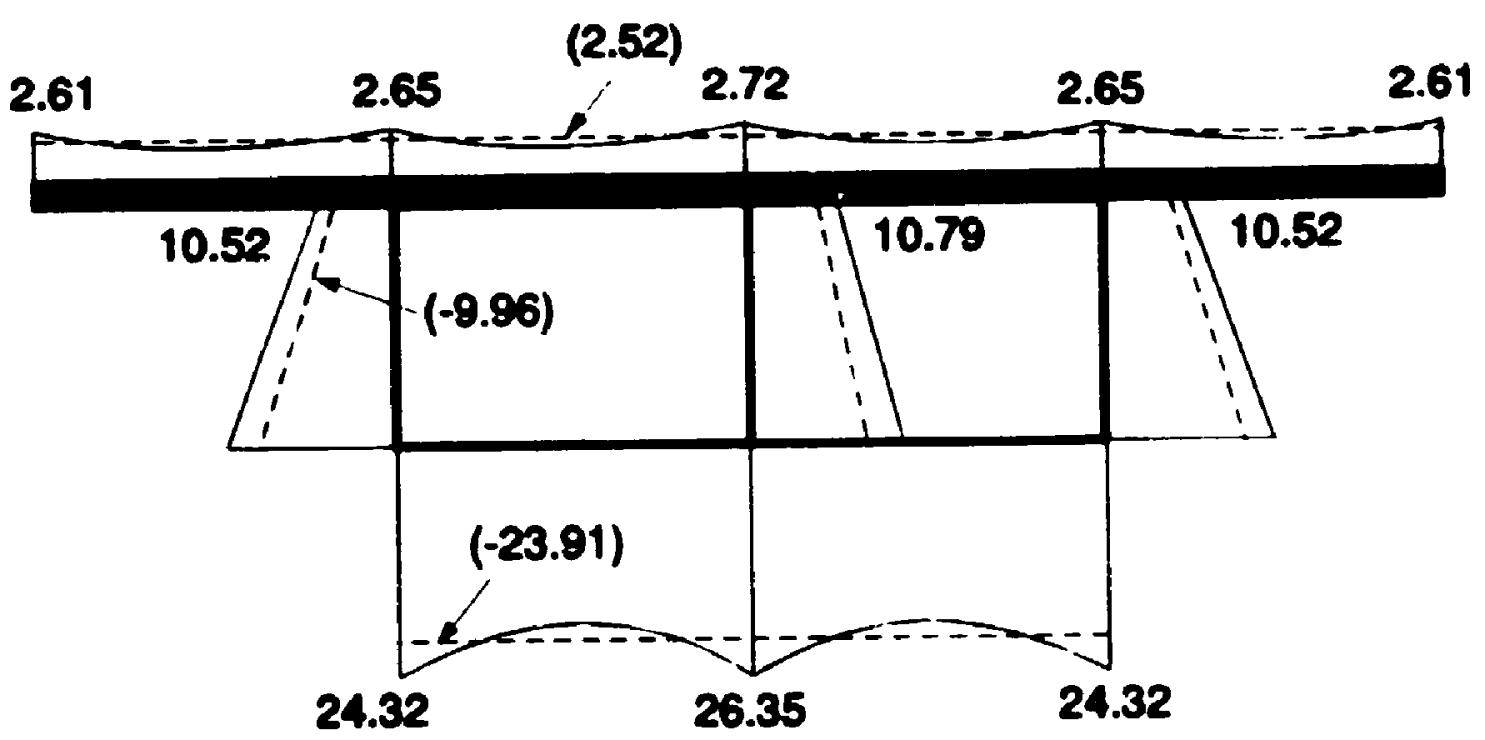

(b). At mid-support.

Fig. 6.41 Longitudinal normal stress distribution due to shrinkage (MPa). 
[8.41] Streas without creap offect

7.75 Stress with creep effect

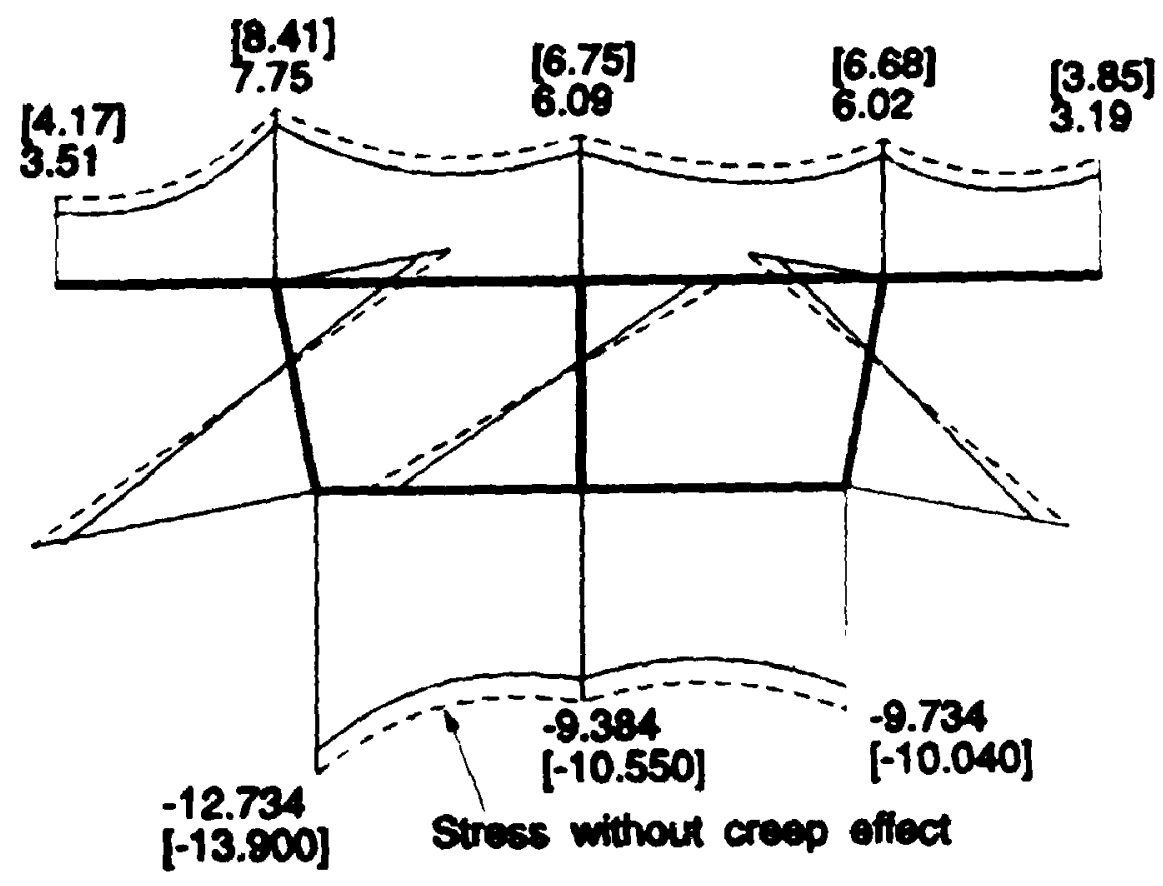

(a). Ar midspan.

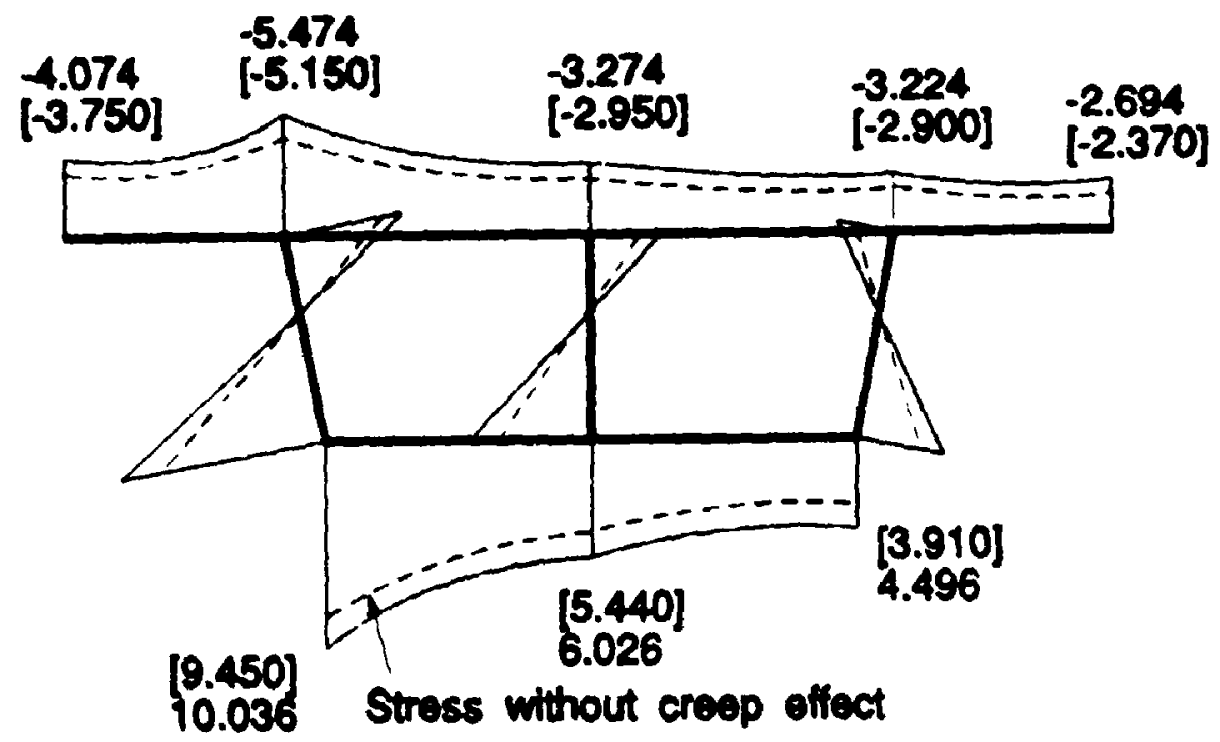

(b). At mid-support.

Fig. 6.42 Longitudinal normal stress distributions due to creep (MPa). 
The various applications presented in this chapter demonstrate that the proposed one-dimensional finite element models are efficient and accurate for practical purposes. The results have been compared with analytical solutions or with other types of numerical methods such as facet shell element and finite strip methods. Good agreement generally exists between the results obtained. The thin-walled box beam element together with a transverse frame analysis give an economical approach for the analysis of straight or curved box girders with variable cross sections and different boundary conditions. 


\section{SUMMARY, CONCLUSIONS AND RECOMMENDATIONS}

\subsection{Summary}

Two efficient thin-walled box beam finite elements which can model extension, flexure, torsion, torsional warping, distortion, distortional warping and shear lag effects were developed using an extended version of Vlasov's thin-walled beam theory for the analysis of multicell box girder bridges with general geometry.

For straight right multicell box girder structures, an orthogonalization procedure was employed to uncouple the various distortional and shear lag modes. The governing differential equation pertaining to each action was used to derive the exact shape functions and the stiffness matrix and nodal load vector of the element. This exuct shape func ion finite element model eliminates the need for dividing the bridge into many elements in each span. For curved box beam finite element, geometry mapping functions and the conventional polynomial shape functions were used to model bridges of general geometry such as haunched and skew bridges. These finite elements are extended to the analysis of box girder assemblages. The effects of prestressing. temperature, creep and shrinkage were also incorporated in the analysis.

One of the advantages of the present methods is that the different structural actions can be uncoupled. This permits the analyst to study each action separately. resulting in a better insight into the behaviour of such structures. The computer resource requirements of the present methods are small and thus can be easily implemented on practically any microcomputer.

Finally, two computer programs SBOXEF and CBOX have been developed based 
on these finite element models , and are used to perform linear elastic analyses of an extensive range of box girder bridges. the results of which are compared with other alternative numerical methods, such as shell finite element and finite strip method. and published experimental or prototype measurements to check the applicability and validity of the methods of analysis presented in this study, with acceptable agreement between the results of the proposed elements and those obtained by the other methods.

\subsection{Conclusions}

1. The assumption that a wide range of multicell, multispan curved, haunched aud skew box girder bridges behave as thin-walled box beam structures is slown to be realistic for practical engineering purpose.

2. It has been demonstrated that the finite elements developed in the present study can predict the response of box girder bridges subjected to general loading conditions fairly accurately. The proposed finite element can acrommodate complex geometry and boundary conditions efficiently. The stress and deformational behaviour of such structures for different loading conditions can be obtainted rapidly.

3. For multi-branch box girder assemblages, the proposed method produced acreptable results over most of the structures except near the junction of intersection which is not unexpected because the basic theory upon which the present formulation is based can not account for such local effects.

4. Prestressing and temperature effects are taken into account in the present analysis. For unsymmetric temperature distribution, torsional and distortional warping would be introduced in box girder structures, and the proposed elements can account for this.

5. Ignoring than-walled beam action can in some cases introduce errors of the order of $38 \%$ in some stresses. 


\subsection{Recomnıendations for Further Research}

1. The current study was restricted to box girder bridge structures. But the proposed finite elements can be extended to general thin-walled beam structures, such as shear walls in tall buildings, aircraft and ship structures, with some nucdifications.

2. The computer programs based on the present finite element models can be incorporated with CAD packages to fully automate the design of box girder bridges. Some more parametric studies are, however, needed to further verify the limitations of the proposed method.

3. The finite element formulation can be used to derive the mass matrix for box girder structures. The dynamic analysis of box girder bridges subjected to earthquake load and the dynamic load introduced by the moving vehicles could be determined using the proposed finite elements.

4. Although geometric nonlinearity is not important in concrete and prestressed concrete box girder bridges, the problem of local buckling for composite box girders and geometric nonlinearity for cable stayed box girder bridges can be considered by the present finite elements. Of course, for steel box girders one could extend the proposed method to account for geometric nonlinearity and st ability analysis.

5. It is suggested that the proposed method be extended to the materially nonlinerr analysis of box girders in order to trace the load-deflection and stress history through the elastic, inelastic and ultimate state ranges. The unbonded prestressing tendons can also be included in this nonlinear analysis. The influence of temperature variation on creep strains may also be incorporated. 


\section{REFERENCES}

(1) American Association of Highway and Transportation Officials (AASHTO), (1989). "Standard Specifications for Highway Bridges." 13th Edition, Washington D.C.. ISA.

[2] American Concrete Institute (ACI), (1982). "Building Code Requirements for Reinforred Concrete (ACI 209-82)." Det roit, Michigan, USA.

[3] Argyris. J.H., Pister, K.S., Szimmat, J. and Willam. K.J. (197i). "I'nified ('oncepts of Constitutive Modelling and Numerical Solution Methods for Concrete Creep Problems". Comput. Meth. in Applied Mech. \& Engrg., 10(2), 199-246.

[4] Bakht, B. and Jaeger, L.S. (1985). "Bridge Analysis Simplified," Mrciraw-Hill Boox Company, New York.

[5] Bakht, B., Jaeger, L.S., Cheung, M.S. and Mufti, A.A. (19K1). "The State of the Art in Analysis of Cellular and Voided Slab Bridges", ('an. J. Civ. Engrg. 8, 376-391.

[6] Bazant, Z.P. and Nimeiri, M.EL (19-4). "Stiffness Method for ('urved Hox Girders at Initial Stress”, J. of Struct. Engrg., AS('E, 100(ST 10), 2071.2090.

[7] Benscoter, S.U. (1954). "A Theory of Torsion Bending for Multicell Beams", J. of Applied Mechanics, 21(1), 25-34.

[8] Branco, F.A. and Green, R. (1985). "Composite Box (iirder Bridge Helaviour During Construction", J. of Struct. Engrg., ASCE, 111(3), 577-593.

[9] British Standards Institution. "Stel, Concrete and Composite Bridges," london, HS5400, Parts 1, 2, 4, 7, 8: 1978, Part 5: 1979.

[10] Chan, M.Y.T., Cheung, M.S., Beauchamp, J.C. and Hachem, H.M. (1990). "Thermal Stresses in Composite Box Girder Bridges", Developments in Short and Medium Span Bridge Engineering 90, (Edited by B. Bakht, R.A. Dorton and L.(i. Jaeger), Toronto, Vol. 2, 355-366.

[11] Chang, S.T. and Ding, Y. (1989). "Shear Lag Effect in Box Girder with Varying Depth", J. of Struct. Engrg., ASCE, 114(10), 2280-2292.

[12] Cheung, M.S., Bakhi, B. and Jaeger, L.G. (1983). "Analysis of Box-Girder Bridges by Grillage and Orthotropic Plate Method", Can. J. Civ. Engrg., O(4), 595-601. 
[1:3] ('heuлg. Y.K. (1976). "Finite Strip Method in Structural Analysis." Pergamon Internalional Library, Oxford.

[14] Churchward, A, and Sokal, Y.J. (1981). "Prediction of Temperatures in Concrete Bridges”, J. of Struct. Engrg., ASCE, 107(ST1! . 2163-2176.

[1:)] ('hu, K.H. and Pinjarkar, S.G. (1971). "Analysis of Horizontally Curved Box Girder Bridges", J. of Struct. Engrg., ASCE, 07(10), 2481-250.

[16] Computer Aided Structural Technology (CAST), (1986). "M - STRUDL User's Manual," Fremont, USA.

[17] Cook, R.D. and Young, W.C. (1985). "Advanced Mechanics of Material," Macmillan Publishing Company, New York.

[18] Choudhury, D. (1986). "Analysis of Curved Nonprismatic Reinforced and Prestressed roncretc Girder Bridges," Ph. D. Dissertation, Division of Structural Engineering and Structural Mechanics, liniversity of California, Berkeley, UC-SESM report No. 86-13.

[19] Dabrowski, R. (1968). "Curved Thin.Walled Girders, Theory and Analysis", C\&CA, London.

[20] Dabrowski, R. (1965). "Approximate Analysis of the Curved Box Girders of Deformable C'ross-Section", 7th IA BSE Congress, Preliminary Publication, Zurich, Switzerland, 299. 306.

[21] Dabrowski, R. (1965). "Warping Torsion of Curved Box Girders of Non-Deformable Cross-Section”, Der Stahlbau, 34(5), 135-141.

[22] Dabrowski, R. (1964). "The Analysis of Curved Thin-Walled Girders of Open Section", Der Stahlbau, 33(12), 364-372.

[23] Dalen. V.K. (1980). "Elastic Behaviour of Composite Box Girder Bridges", Can. J. (iv. Eingrg., $\mathrm{T}(3), 492-501$.

[24] Dalen. V.h. and Narasimham, S.V. (1976). "Shear Lag in Shallow Wide-Flanged Box (iirders"., J. of Struct. Engrg., ASCE, 102(10), 1969-1979.

[25] Dezi, I. and Mentrasti, L. (1984). "Nonuniform Bending Stress Distribution (Shear Lag)“. J. of Struct. Engrg., ASCE, 111(12), 2675-2690.

[26] Dilger, W.H.. Ghali, A.. Chan, M., Cheung, M.S. and Maes, M.A. (1983). "Temperature Stresses in Composite Box Girder Bridges", J. of Struct. Engrg.. ASCE, 109(6), 1460 1478. 
[27] Elbadry, M.M. and Ghali. A. (1983). "Temperature Variations in Concrete Bridges", $J$. of Strcut. Engrg., ASCE, 109(10), 2355.2374.

[28] Fam, A.R.M. and Turkstra, C. (1975). "A Finite Element Scheme for Box Bridge Analysis", J. of Comput. \& Struct., B(2/3), 179-186.

[29] Foutch, D.A. and Chang. P.C. (1982). "A Shear Lag Anomaly", J. of Strurt. Engrg., ASCE, 108( 7$), 1653-1658$.

[30] Ghali, A. and Neville, A.M. (1989). "Structuml Analysis," 3rd edition, Chapman and Hall, New York.

[31] Ghali, A. and Favre, R. (1986). "Concrete Structures: Stresses and Deformutions." Chapman and Hall, New York.

[32] Ghoneim, G.A. and Ghali A. (1982). "Nonlinear Analysis of Concrete Structures", ('an. J. Civ. Engrg., $9(3), 489-501$.

[33] Gunnlaugsson, G.A. and Pedersen, D.T. (1982). "A Finite Elems st Formulation for Beams with Thin Walled Cross-Section", J. of Comput. \& Struct., 15(6), 691-699.

(34] Hajdin, N. and Dunica, S. (1974). "Folded Plates Stiffened By Diaphragms," IASS Sym. posium on Folded Plates and Spatial Panel Structures, Udine, Italy, Preliminary Report.

[35] Heins, C.P. and Oleinik, J.C. (1976). "Curved Box Beam Bridge Analysis", J. of Comput. \& Struct., $6(2), 65-73$.

[36] Hetenyi, M. (1961). "Beams on Elastic Foundation," The University of Michigan Press, An Arbor.

[37] Jaeger, L.S. and Bakht, B. (1989). "Bridge Analysis by Microcompuler," Mc(iraw-llill Book Company, New York.

[38] Jaeger, L.S. and Bakht, B. (1982). "The Grillage Analogy in Bridge Analysis", Can. J. Civ. Engrg., 9, 224-235.

[39] Jirousek, J. and Bouberguig, A. (1979). "A Macro-Element Analysis of Prestressed Curved Box-Girder Bridges", J. Comput. \& Strurt., 10, 467-482.

[40] Kabir, A.F. (1976). "Nonlinear Analysis of Reinforced Concrete Pancls, Slabs and Shells for Time-Dependent Effects," Ph. D. Dissertation, Division of Structural Engineering and Structural Mechanics, University of California, Berkeley, UC.SESM report No. 76-6. 

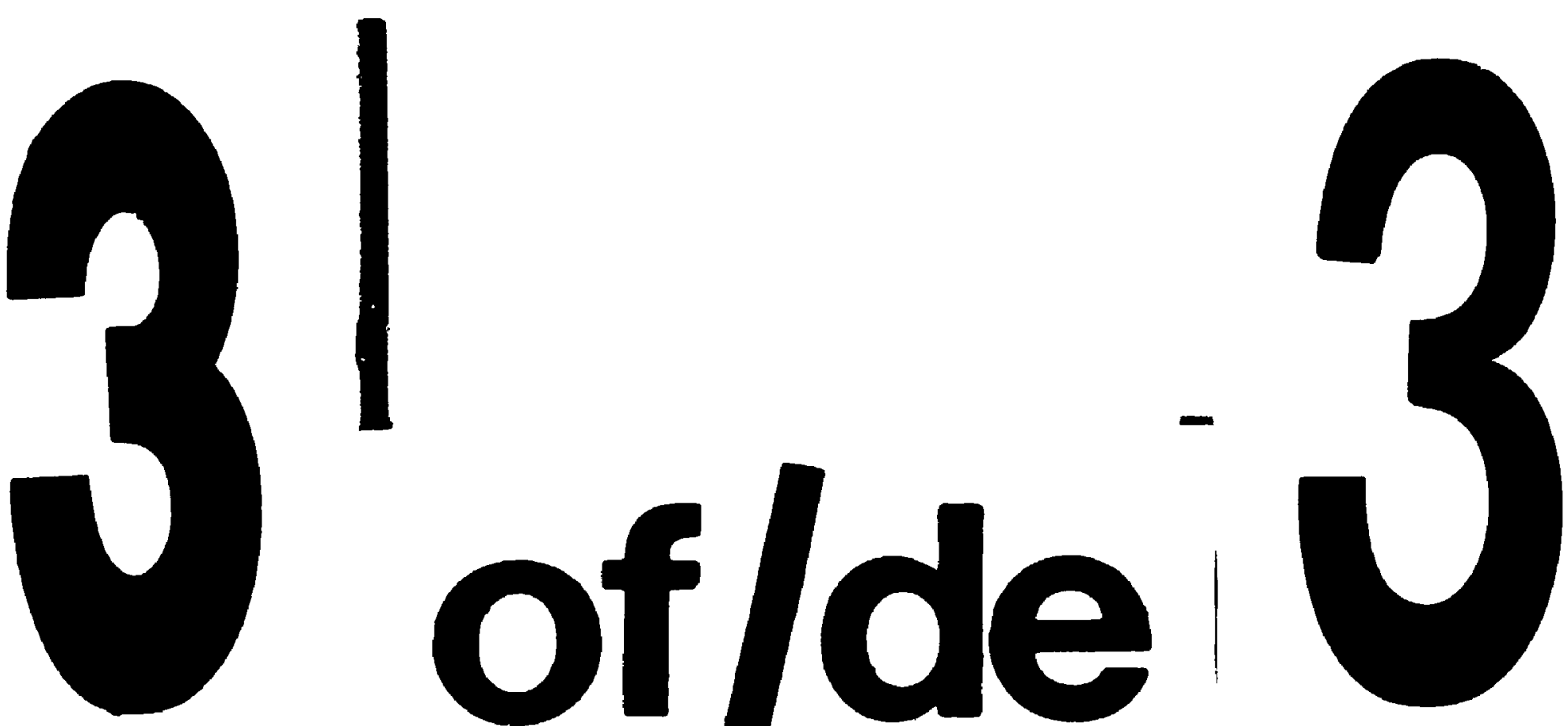

PM-1 3K" $\times 4$ " PHOTOCRAPHIC MICAOCOPY TARCET NBS 10t0a AMSH/LO w2 ECUNALENT

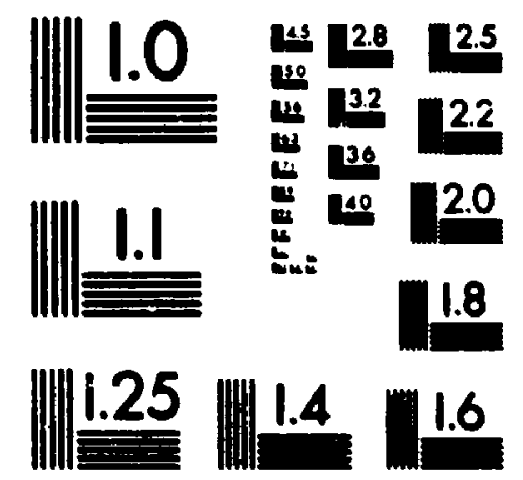

PRECISION RESOLUTION TAROETS

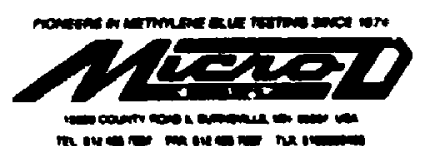


[41] Kollbrunner, C.F. and Hajdin, N. (1975). "Thin-Halled Beams," Vol. 2, Berlin, Springer. Verlag. 284 pp.

[42] Kollbrunner, C.F. and Hajdin, N. (1966). "Warping Torsion of Thin-llalled Beams of Closed Section," Zurich. Verlag Schweizer Stahlbau-Vereinigung. Mitteilungen der Ter. nischen hommission, Heft 32. 175 pp.

[43] Krajcinovic, D.K. (1969). "A Consistent Discrete Elements Technique for Thin-Walled Assemblages", Int. J. of Solids and Struct., 3, 639-662.

[44] K'ristek, V. anó Bazant, 2.P. (1987). "Shear Lag Effect and Uncertainty in Concrete" Box Girder Creep”, J. of Struct. Engrg., ASCE, 113(3), 557-574.

[45] Kristek, V. (1970). "Tapered Box Girders of Deformable Cross Section". J. of Struct. Engrg., ASCE, 86(ST8), 1761-1793.

[46] Li, G.H. (1987). "Analysis of Box Girder and Truss Bridges," Springer-Verlag, Berlin, China Academic Publishers, Beijing.

[47] Li, W.Y., Tham, L.G. and Cheung, Y.K. (1988). "Curved Box-Girder Bridges", J. of Struct. Engrg., ASCE, 114(6), 1324-1338.

[48] Loo, Y.C. and Cusens, A.R. (1978). "The Finite Strip Method in Bridge Engineering." Wexham Springs, Viewpoint Publications, 220 pp.

[49] Lyons, L.P.R. (1977). "A General Finite Element System uith Special Reference to the Analysis of Cellular Structures," Ph. D. Thesis, Imperial College of Science and Technology, London, England.

[50] Maisel, B.I. (1986). "Shear Lag Analysis of Concrete Box Beams Using Small Computer Capacity", Proceedings of the Second International Conference on Short and Medium Span Bridges, Ottawa, Canada, 125-137.

[51] Maisel, B.I. (1982). "Analysis of Concrete Box Beams Using Small-Computer Capacity", C\&CA, Development Report 5, London.

[52] Maisel, B.I., ETAL (1973). "Concrete Box-Girder Bridges", The Strurtural Engineer, $51(10)$.

[53] 1. - I, B.I. (1970). "Review of Literature Related to the Analysis and Design of ThinWalled Beams", C\&CA. Technical Report 110, London.

[54] Mavaddat, S. and Mirza, M.S. (1989). "Computer Analysis of Thin-Walled Cocrete Box Beams”, Can. J. Civ. Engrg., 16(12), 902-909. 
[5.5] Meyer, (. (1970). "Analysis and Design of Curved Box Girder Bridges," Berkeley, University of California, Department of Civil Engineering, Report No. UC SESM 70-22.

[56] Meyer, C. and Scordelis, A.C. (1971). "Analysis of Curved Foldec !'late Structures", J. of Sitruct. Engrg., ASCE, $07(10)$, 2459-2479.

[57] Mikkola, M.J. and Paavola, J. (1980). "Finite Element Analysis of Box Girders", J. of Struct. Engrg, ASCE, $106($ ST6), 1343-1356.

[58] Moffatt, K.R. and Lim, P.T.K. (1976). "Finite Element Analysis of Composite Box Girder Bridges Having Complete or Incomplete Interaction", Procecaings of the Institution of Civil Engineers, Part 2: Research and Theory, 61(3), 1-22.

[59] Moffat, K.R. and Dowling, P.J. (1978). "The Longitudinal Bending Behaviour of Composite Box Girder Bridges Having Incomplete Interaction", The Structural Engineer, $56 B(3), 53-60$.

[60] Moffatt, K.R. and Dowling, P.J. (1978). "British Shear Lag Rules for Composite Girders”, J. of Struct. Engrg.,, ASCE, 104(ST7), 1123-1130.

[61] Moffatt, K.R. and Dowling, P.J. (1975). "Shear Lag in Steel Box Girder Bridges", The Structural Engineer, 53(10), 439-448.

[62] Moore, T.A. (1975). "Finite Element Analysis of Box-Girder Bridges" Ph. D. Thesis, Department of Civil Engineering, University of Canterbury, Christchurchs New Zealand.

[63] Moorty, S. and Roeder, C.W. (1990). "Thermal Response of Skewed Bridges", Developments in Short and Medium Span Bridge Engineering' 90, (Edited by B. Bakht, R.A. Dorion and L.G. Jaeger), Toronto, Vol. 2, 343-353.

[64] Nakai, H. and Yoo, C.H. (1988). "Analysis and Design of Curved Steel Bridges," McGraw Hill, New York.

[65] Oden, J.T. and Ripperger, E.A. (1981). "Mechanics of Elastic Structures," 2nd Edition, McGraw-Hill, New York.

[66] Ontario Ministry of Transportation and Communications, (1983). "Ontario Highway Bridge Design Code," (OHBDC), Ontario, Canada.

[67] Prakash, R.D.S. (1986). "Temperature Distributions and Stresses in Concrete Bridges", J. of $A C l .83(4), 588-596$.

[68] Prasada. R.A.S. and Jayaraman, R. (1989). "Creep and Shrinkage Analysis of Partially Prestressed Concrete Members", J. of Struct. Engrg., ASCE, 115(5), 1169-1189. 
[69] Priestley, M.J.N. (1976). "Design of Thermal Gradients for Concrete Bridges". Neu' Zealand Engineering, 31(9), 213-219.

[70] Razaqpur, A.G. and Li, H.G. (1991). "Thin-Walled Multicell Box Girder Finite Element", J. of Struct. Engrg., ASCE, 117(10), 2953-22971.

[71] Razaqpur, A.G. and Li, H.G. (1991). "A Finite Element with Exact Shape Functions for Shear Lag Analysis in Multi-Cell Box Girders”, J. of Computer and Struct., 30(1/2). $155-163$.

(72) Razaqpur, A.G. and Li, H.G. (1990). "Analysis of Multi-Branch Multi-Cell Box Girder Bridges", Developments in Short and Medium Span Bridge Engincering ${ }^{\circ}$ 90, (Edited by B. Bakht, R.A. Dorton and L.G. Jaeger), Toronto, 153-164.

[73] Razaqpur, A.G. and Li, H.G. (1990). "Finite Element Formulation of Distortional Warping in Multi-Cell Box Girders", Developments in Structural Engineering, (Edited by B.H.V. Topping), Chapman and Hall, London, 1261-1270.

[74] Razaqpur, A.G. and Shah, K.R. (1990). "Exact Analysis of Beams on Two-Parameter Elastic Foundations”, International J. of Solids and Struct., 27(4), 435-454.

[75] Razaqur, A.G., Nofal, M. and Mirza, M.S. (1989). "Nonlinear Analysis of Prestressed Concrete Box Girder Bridges under Flexure”, Can. J. Civ. Engrg., 16(12), 845-853.

[76] Razaqpur, A.G. (1986). "Stiffness of Beam-Columns on Elastic Foundation with Exact Shape Functions", J. of Comput. \& Struct., 24(5), 813-819.

[77] Razaqpur, A.G. (1986). "Forces at the Slab-Web Connections of Box-Girders", Proceedings of the Second International Conference on Short and Medium Span Bridges, Ottawa, Canada, 193-203.

[78] Reissner, E. (1938). "On the Problem of Stress Distribution in Wide-Flanged Boxbeams", J. Aero. Sci., 5(8), 295-9.

[79] Roik. K. and Sedlacek, G. (1966). "Theory of Warping Torsion Considering Secondary Shear Deformation Analogy with Analysis of the Transversely Loaded Beam in Torsion", Der Stahlbau, 35(2), 43-52 (in German).

[80] Roik, K. and Sedlacek, G. (1970). "Extension of Engineer's Theory of Bending and Torsion, Considering Shear Deformation”, Die Bautechnik, 47(1), 20-32 (in (ierman).

[81] Sawko, F. and Cope, R. J. (1970). "Analysis of Spine Beam Bridges Using Finite Elements" Civil Engineering and Public Works Review, Vol. 65, No. 753, pp. 146-147. 
[\$2] Schlaich, J. and Scheef, H. (1981). "Concrete Box Girder Bridges," Structural Engineering Documents-1e, International Association for Bridge and Structural Engineering. Zurich.

[83] Scordelis, A.C. (1982). "Berkeley Computer Programs for the Analysis of Concrete Box Girder Bridges", Proceedings of NATO-Advanced Study Institute on Analysis and Design of Bridges, Cesme, Turkey.

[84] Scordelis, A.C., Wasti, S.T. and Seible, F. (1982). "Structural Response of Skew RC Box Girder Bridge”, J. of Struct. Engrg., ASCE, 108(ST1), 89-104.

[85] Scordelis, A.C. and Larsen, P.K. (1977). "Structural Response of Curved RC Box-Girder Bridge", J. of Struct. Engrg., ASCE, 103(ST8), 1507-1524.

[86] Scordelis, A.C. (1974). "Folded Plates for Bridges", General Report, Session IV, Symposium on Folded Plates and Spatial Panel Structures, Udine, Italy, 1974. Bulletin of the International Asscoiation for Shell and Spatial Structures. Vol. XVI-1, No. 57, 29-38.

[87] Scordelis, A.C., Bouwkamp, J.G. and Wasti, S.T. (1973). "Structural Response of Concrete Box Girder Bridge”, J. of Struct. Engrg.,, ASCE, 89(ST10), 2031-2048.

[88] Scordelis, A.C. (1971). "Analytical Solutions for Box Girder Bridges", Developments in Bridge Design and Construction, (Edited by K.C. Rockey, J.L. Bannister and H.R. Fvans), Crosby Lockwood and Son, London, 200-216.

[89] Scordelis, A.C. (1967). "Analysis of Continuous Box Girder Bridges," Berkeley, University of California, Department of Civil Engineering, Report No. UC SESM 67-25.

[90] Scordelis, A.C. (1966). "Analysis of Simply Supported Box Girder Bridges," Berkeley, University of California, Department of Civil Engineering, Report No. UC SESM 66-17.

[91] Sedlacek, G. "Systematic Description of the Process of Bending and Torsion for Prisnuatic Beams of Thin-Walled Cross-Section, Considering Distortion of Cross-Section", Fortschritt-Berichte VDI-Zeitschrift, Reihe 4, Nr. 8, September 1968, 110pp (in Gernam).

[92] Shiu, K-N and Russell, H.G. (1987). "Effects of Time-Dependent Concrete Properties on Prestress Losses”, Can. J. Civ. Engrg., 14(5), 649-654.

[93] Sisodiya, R.G. and Ghali, A. (1973). "Analysis of Box Girder Bridges of Arbitrary Shape", IABSE Publications, 33-I, 203-218.

[94] Tadros, G., Ghali, A. and Dilger, W. (1982). "Construction Stages and Design Consid- 
erations for Curved Box-Girder Bridges”. Can. J. Civ. Engrg., 9(3), 483-488.

[95] Timoshenko, S.P. (1905). "On the Stability in Plane Bending of an I-Beam". Izvestiya St. Petersburg Politekhnicheskogo Instituta, IV.V.

[96] Vlasov, V.Z. (1961). "Thin-Walled Elastic Beams," Washington, D.C.. National Science Foundation.

[97] Waldron, P. and Ramezankhani, M. (1990). "Differential Temperature Effects in Con. crete Box Girder Bridges", Developments in Short and Medium Span Bridge Engineering ${ }^{\circ}$ 90, (Edited by B. Bakht, R.A. Dorton and L.G. Jaeger), Toronto, Supplement 1-12.

[98] Waldron, P. (1988). "The Significance of Warping Torsion in the Design of Straight Concrete Box-Girder Bridges", Can. J. Civ. Engrg., 15, 879-889.

[99] Waldron, P. (1986). "Stiffness Analysis of Thin-Walled Girders", J. of Strurt. Engrg., ASCE, $112(6)$, 1366-1384.

[100] Wright, R.N., Abdel-Samad, S.R. and Robinson, A.R. (1968). "BEF Analogy for Analysis of Box Girders”, J. of Struct. Engrg., ASCE, 84(ST7), 1719-1743.

[101] Yoo, C.H. (1980). "Bimoment Contribution to Stability of Thin-Walled Assemblages", J. of Comput. \& Struct., 11, 465-471.

[102] Zhang, S.H. and Lyon, L.P.R. (1984). "The Application of the Thin-Walled Box Beam Element to Multibox Bridge Analysis”, J. of Comput. \& Struct., 18(5), 795-802.

[103] Zhang, S.H. and Lyon, L.P.R. (1984). "A Thin-Walled Box Beam Finite Element For Curved Bridge Analysis”, J. of Comput. \& Struct, 18(6), 1035-1046. 


\section{APPENDIX A: COMPUTER PROGRAMS AND USER MANUAL}

\section{GENERAL}

Two computer programs SBOXEF and CBOX have been developed to carry out the analysis of box girder structures utilizing the methods which have been described in this thesis. The programs are used for linear elastic analysis of reinforced concrete, prestressed concrete and composite multicell box girder structures. The influence of torsional warping, distorion and distortional warping, shear lag and shear lag warping, which can not be predicted in conventional engineer's beam theory on beam stresses, can be investigated. The response of the structure due to temperature, and time-dependent effects, such as shrinkage and creep, are simulated in program CBOX.

The programs are coded in FORTRAN 77 and have been tested on the APPOLO and SUN workstations. An overlay structure is employed for the program and the blank common dynamic dimension system is used within the programs in order to fit the memory requirements of the problem under consideration. This makes the efficient use of the avalable central memory capacity of the computer possible and thus minimizes computer expenses.

The programs are structured such that additional elements could be easily added in the future. The equation solving is done by Gaussian elimination and the equation solver adapted solves symmetric banded matrices by triangularization and then back substitution is performed in two separate operations. The size of structures that can be analysed is dependent on the size of core storage of the computer being used.

The data input is in free format and thus suitable for input from a terminal. It is, however, very easy to modify the input statements should one wish to input 
formatted data. Some automatic data generation facilities are availnble, which enable considerable economy in data input. The output of the programs is clear and selfexplanatory. The programs accept any consistent set of units.

\section{PROGRAM SBOXEF}

Computer program SBOXEF (straight box girder analysis with exact shape functions) has been developed based on the theory of the finite element described in Chapters 2 and 3 of this thesis. The straight multicell box beam finite element has two nodes similar to a conventional beam element. In addition to the six degrees of freedom in conventional beam element for each node, more degrees of freedom for torsional warping, distortion and distortional warping, shear lag and shear lag warping are included in order to model the complex behaviour of multicell box bridges under general loading conditions.

The program is limited to linear elastic analysis for right prismatic box girders and does not account for local effects. The program also does not include cross sectional distortions caused by symmetrical loads applied between the webs. This effect can be, however, approximately accounted for by analyzing a unit length of the box as a Vierendeel frame.

\section{INPUT DATA INSTRUCTIONS FOR SBOXEF}

The input data cards required to run program SBOXEF are described below in the order in which they should be input. It is recognized that a more detailed users manual will be needed to fully describe the input and output of the program. The format used to read the data from cards is enclosed between square brackets. Consistency in units used must be observed in the input data except where otherwise indicated. Although specified input format will be indicated in the following 
instrurtions, the data input is in free-format.

1. $[20 \mathrm{A4}]$

2. [413]

NODE

NMEM

NCELL

NCAN

3. [2F10.3]

$\mathrm{XY}(\mathrm{I}, 1)$

$\mathrm{XY}(1,2)$

4. $[213,3 F 10.3]$

$\operatorname{MEM}(\mathbf{I}, \mathbf{J})$

ET( $(I, 1)$

ET(I,2)

ET(1,3)

5. [413]

$\operatorname{MCEL}(\mathbf{I}, \mathrm{J})$

6. [I3,F10.3]

NELM

GAMMA
: one card including the title of the problem to be printed with output for identification,

: one control card for cross section information including: = number of nodal points of the box cross section, $=$ total number of walls of the cross section, $=$ number of cells of box girder, = information for overhangs of the cross section, $=0$ no overhang for cross section, $=1$ consider overhang for cross section.

: a number of cards equal to the number of nodal points NODE including:

$=\mathrm{X}$-coordinate of node $\mathrm{I}$.

$=\mathrm{Y}$-coordinate of node $\mathrm{I}$,

: a number of cards equal to the number of walls NMEM including:

$=$ nodes 1 and 2 of wall $\mathrm{I}(\mathrm{J}=1,2)$,

$=$ thickness of wall I,

= Young's modulus of wall I,

$=$ shear modulus of wall $\mathrm{I}$,

: a number of cards equal to the number of cells, NCELL, in the box cross section, $=$ plate numbers of cell $\mathrm{I},(\mathrm{J}=1$ to 4$)$,

: one control card for longitudinal element information including:

$=$ total number of elements in longitudinal direction, $=$ density of material for self-weight anaiysis,

Items (2) to (6) are the properties of cross section which are defined in Fig. (A.1).

i. $[F 10.3,313] \quad:$ a number of cards equal to the number of elements NELM, including: 
SL(I) = length of element I.

IELM(I.1) = number of uniformly dist ributed load acting on element 1.

$\operatorname{IELM}(I .2) \quad=$ number of concetrated load acting on element $I$.

$\operatorname{IELM}(1,3) \quad$ = number of cross sections for which stresses must be output.

8. [F10.3] : for each element. a number of cards equal to the number of cross sections for output stress distribution including:

$\mathrm{XPCH}(\mathrm{I} . \mathrm{J})=z$-coordinate of cross section $\mathrm{J}$ in element $\mathrm{I}$,

Items ( $\bar{c}$ ) and (8) are the information of longitudinal elements.

9. [2F10 3] : for each element, a number of cards equal to the numler of uniformly distributed load (can be omitted if no such load) including:

$\mathrm{QL}(\mathrm{I}, \mathrm{J}, 1) \quad=$ value of uniformly distributed load $\mathrm{J}$ for eiement $\mathrm{I}$.

$\mathrm{QL}(\mathrm{I}, \mathrm{J}, 2) \quad=$ transverse distance from the origin of cocordinate system in cross section,

10. [3F10.3] : for each element, a number of cards equal to the number of of concetrated load (can be omitted if no such load) including:

$\mathrm{PL}(\mathrm{I}, \mathrm{J}, 1)=$ value of conentrated load $\mathrm{J}$ for element $\mathbf{I}$,

$\mathrm{PL}(\mathrm{I}, \mathrm{J}, 2) \quad=$ transverse distance from the origin of coordinate system to the point of application of the load,

$\mathrm{PL}(\mathrm{I}, \mathrm{J}, 3) \quad=$ longitudinal distance from the origin of cocordinate syste'm to the point of the application of the load,

Items (9) and (10) are the loading information.
11. $[13$, F10.3 $]$
: a number of cards of boundary conditions for distortional analysis including, (the last one must only contain 0,0 ):
$=$ global degree of freedom number with ronstrained displacr
$=$ value of constrained displarment,
12. $[13$, F10.3]
: a number of cards of boundary conditions for shear lag analys: 3 including, (the last one must only contuin 0,0 ):
$=$ degree of freedom with constrained displarment,
$=$ constrained displacment. 
Items (11) and (12) are boundary conditions for distortional and shear lag analysis. The boundary conditions for flexure are the same as those for shear lag analysis.

\section{PROGRAM CBOX}

Computer program CBOX (curved box girder finite element analysis) has been developed based on the theory and the finite element procedures described in Chapters 4 and 5 of this thesis. It can be used for general curved multicell box girder bridge analysis, including prestressing, temperature and time-dependent effects. The curved multicell box beam finite element has three nodes and it uses the Timonshenko's beam theory to include the shear deformations. Again, in addition to the degrees of freedom in conventional curved beam element, more degrees of freedom for torsional warping, distortion and distortional warping, shear lag and shear lag warping are included. Polynomial shape functions and Gaussian numerical integrations are used to calculate the element stiffness matrix and equivalent load vector.

For thermal stress analysis, Priestly's temperature distribution is used in the program. In the shrinkage and creep model, the ACI (1982) formulas are used. However, it is not difficult to modify the program for other temperature distributions and shrinkage and creep models.

The program is limited to linear elastic analysis, and it does not account for bond slip between concrete and the prestressed cables. It also does not account for prest ress losses.

\section{INPUT DATA INSTRUCTIONS FOR CBOX}

The input data cards required to run program CBOX are described below in the order in which they should be input. The format used to read the data from cards is enclosed between square brackets. Consistency in units used must be observed in the 
input data except where otherwise indicated. Although specified input format will be indicated in the following instructions. the data input is completely free-format in the present program.

1. [20A4]

2. [2I3, F10.3]

NELM

NTHE

GAMMA

3. $[913,4 \mathrm{~F} 10.3]$

$\operatorname{INDEX}(1)$

$\operatorname{IELM}(\mathbf{I}, \mathbf{K})$

$\operatorname{IELM}(I, 3)$

$\operatorname{IELM}(I, 4)$

$\operatorname{IELM}(\mathrm{I}, 5)$

$\operatorname{IELM}(1,6)$

$\operatorname{IELM}(1,6)$

$\operatorname{ELM}(I, 7)$

$\operatorname{ELM}(1,8)$

$\operatorname{IELM}(1,8)$
: one card including the title of the problem to be printed with output for identification.

: one control card for element information including:

$=$ total number of elements in longitudinal direction.

$=$ analysis control code.

$=1$ use engineer's beam theory.

$=2$ use Vlasov's thin-walled beam theory:

$=3$ use extended Vlasov's thin-walled beam theory.

$=$ density of material for self-weight analysis,

: a number of cards equal to the number of elenient NELM including:

$=$ index for element $\mathrm{I}$.

$=$ two node numbers of element $I,(K=1,2)$,

$=$ number of uniformly distributed load arting on element. $I$,

= number of concetrated load acting on element 1 ,

$=$ code for prestressing information,

$=0$ no prestressed cable,

= 1 consider prestressing effect,

= code for temperature information,

$=0$ no temperature effert,

$=1$ consider temperaturc effect,

$=$ code for shrinkage information,

$=0$ no shrinkage effect,

$=1$ consider shrinkage effect,

$=$ code for creep information,

$=0$ no creep effect,

$=1$ consider creep effect,

= curved length of element $I$, 


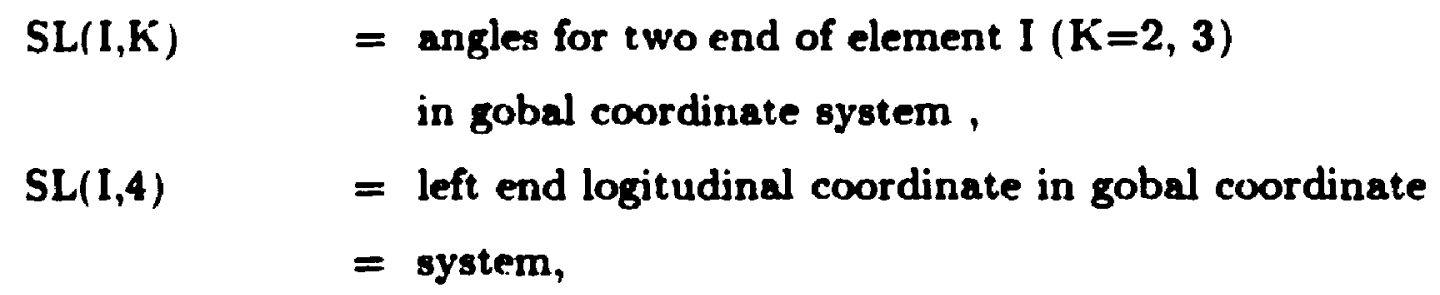

Item (1) to (3) are information for longitudinal finite element.
4. [213]
: number of cards equal to the number of elements NELM includirig:
NCELL = number of cells in the box girder,
NCAN = information for overhangs of the cross section,
$=0$ no overhang in the cross section,
$=1$ there is overhang in the cross section,
5. [6F10.3]
: a number of cards equal to the number of walls, material properties of walls, including:
$\operatorname{ET}(\mathrm{J}, 1)=$ thickness of plate $\mathrm{J}$,
$\operatorname{ET}(\mathrm{J}, 2)=E_{2}$,
$\operatorname{ET}(\mathrm{J}, 3)=E_{9 x}$,
$\operatorname{ET}(\mathrm{J}, 4) \quad=E_{\mathrm{g}}$,
$\operatorname{ET}(\mathrm{J}, 5) \quad=\nu_{2}$,
$\operatorname{ET}(\mathrm{J} .6) \quad=\nu_{9}$,

6. [2F10.3] : a number of cards equal to the number of nodal points, NODE, of the cross section including:

$X Y(J, 1)=X$-coordinate of node $J$,

$X Y(J, 2)=Y$-coordinate of node $\mathrm{J}$,

7. $[$ F10.3, 3I3] : for each element, input vectors $\{a\}$ and $\{b\}$ for each node:
$A B(I .1 . K)=a_{x 1}, a_{y 1}$ and $a_{x i},(K=x, y$ and $z)$,
$A B(I .2, K) \quad=b_{x i}, b_{y i}$ and $b_{x i},(K=x, y$ and $z)$, $(1=1,2,3)$,

Items (4) to $(\bar{z})$ are the properties of cross section. If $\operatorname{INDEX}(I)=\operatorname{INDEX}(\mathrm{I}-1)$, the properties of cross section of this element are the same as those of last element. This group of data can be omitted and would be generated by the program. 
8. [13. 4F10.3] : for each element, a number of cards equal to the number of uniformly distributed loaòs (can be omitted if no such load) including:

IUDF(I) = wall number on which the load is acting.

$\mathrm{UDF}(\mathrm{I}, \mathrm{J})=$ components of the load in directions $z, n$ and s, respectively, $(\mathrm{J}=1,2,3)$,

IDF $(I, 4)=$ transverse distance of the load from the origin of the coordinate system,

9. $[13,5 F 10.3] \quad$ : for each element, a number of cards equal to the number of concetrated loads (can be omitted if no such lond) including:

IPL(I) = wall number on which the load is acting,

PLF(I,J) = components of the concentrated load in directions $z, n$ and $s$, respectively, $(\mathrm{J}=1,2,3)$,

$\operatorname{PLF}(\mathrm{I}, 4) \quad=$ transverse distance of the load for the origin of the coordinate system,

$\operatorname{PLF}(1,5)=$ longitudinal distance of the load,

Items (8) and (9) are information for loading conditions shown in Fig. A.2.

10. $[13,4 F 10.3] \quad$ : for each element, a number of cards equal to the number of prestressed cables (can be omitted if no prestressed cable) including:

IPSF(I) $\quad=$ wall number in which the particular prestressed cable is lorate

$\operatorname{PLF}^{(1,1)} \quad=$ magnitude of the prestressing force,

$\operatorname{PLF}(\mathrm{I}, \mathrm{J}) \quad=\mathrm{y}$-coordinates of cable, $(\mathrm{J}=2,3,4)$, begining, middle and end points (see Fig. A.3),

21. [AFin.3] : for each element, a number of cards equal to the number of walls in the cross section (can be omitted if no temperature effect) including:

$\operatorname{ET12}(\mathrm{I}, 1)=$ temperature of wall $\mathrm{I}$,

ET12(I,2) $=\alpha_{z}$, coefficient of thermal expansion in z-coordinate pertaining to wall I, 
ET12(I,3) $=\alpha_{s}$, coefficient of thermal expansion in s-coordinate pertaining to wall $\mathrm{l}$,

$\operatorname{ET} 12(1,4)=$ type of $\cdots$ perature distribution,

$\operatorname{ET} 12(1,5)=$ order of th tiperature distribution function,

12. $[4 \mathrm{~F} 10.3]$

: for each element, a number of cards equal to the number of wall in the cross section (can be omitted if no shrinkage and creep effect; including:

$\operatorname{ETCS}(I, 1)=$ type of curing method, $=1$ moist-cured concrete,

$=2$ steam-cured concrete,

$\operatorname{ETCS}(I, 2)=t$ in days, age of concrete when the strain is considered,

$\operatorname{ETCS}(1,3)=t_{0}$ in days, age of concrete when the initial stress is applied,

$\operatorname{ETCS}(I, 4)=E_{c}(28)$, concrete modulus of elasticity at age 28 days,

Items (10) to (12) are prestressing, temperature and time-dependent information. Prestressing and temperature information are shown in Figs. A.3 and A.4, respectively.

13. $[213, F 10.3]$ : a number of cards related to the boundary conditions, including: (the last card must only contain $0,0,0$ ):

NWI = node number with constrained displacment,

NWF $\quad=$ degree of freedom number with constrained displacment,

$\mathrm{V}=$ magnitude of the constrained displacment,

Item (13) is the boundary conditions of the structure.

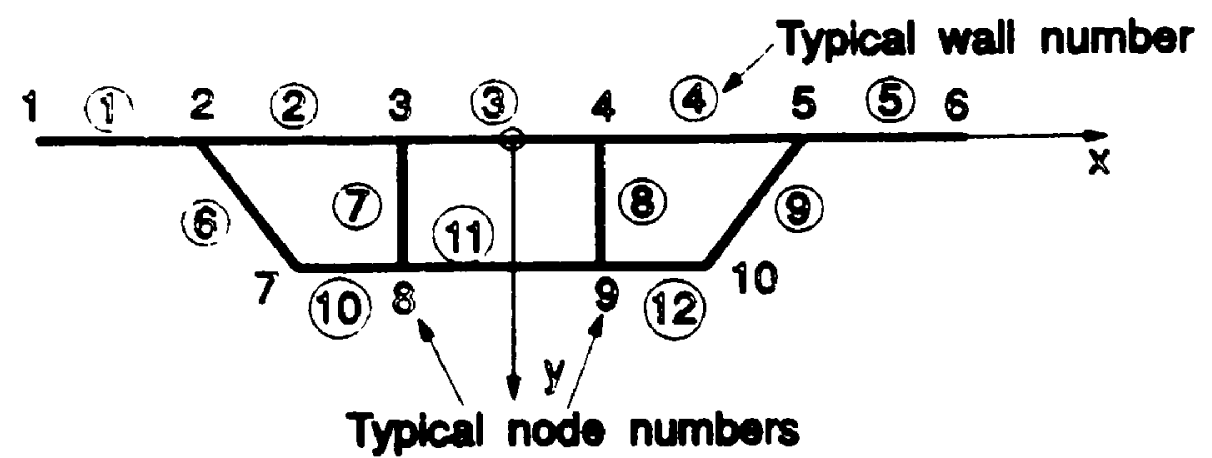

Fig. A.1 Node and wall definition of cross section. 


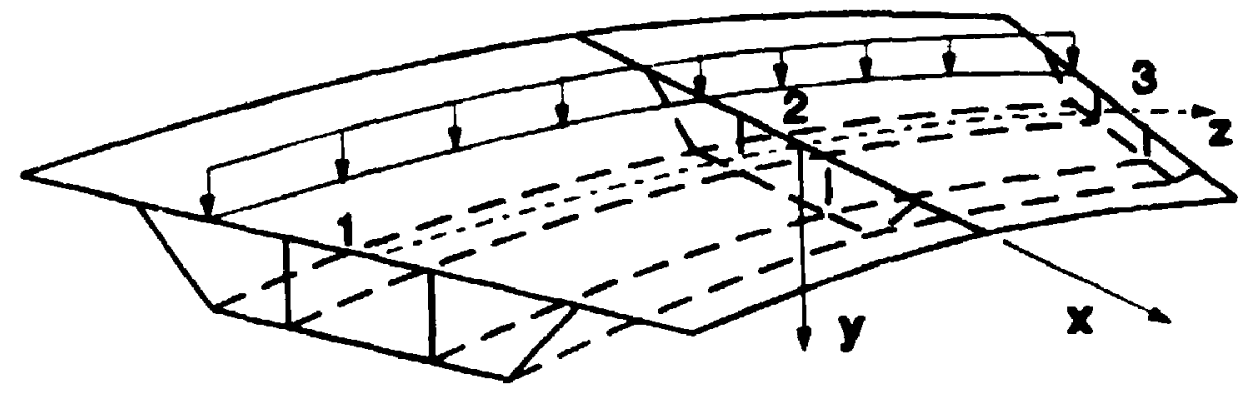

(a) Uniformly distributed load for curved element.

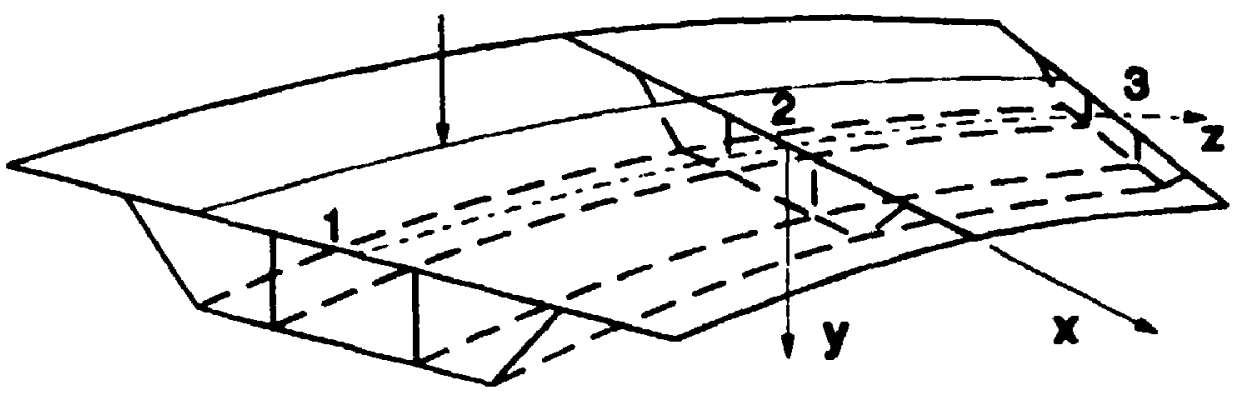

(b) Concentrated load for curved element.

Fig. A.2 Loading information for curved element. 


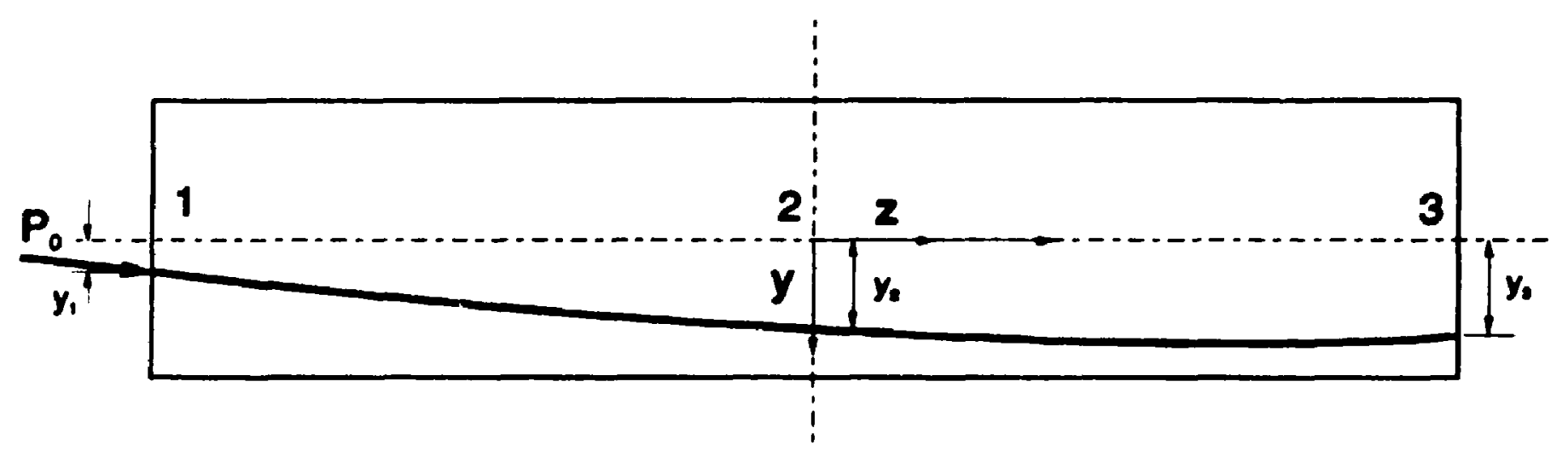

Fig. A.3 Prestressed bar information.

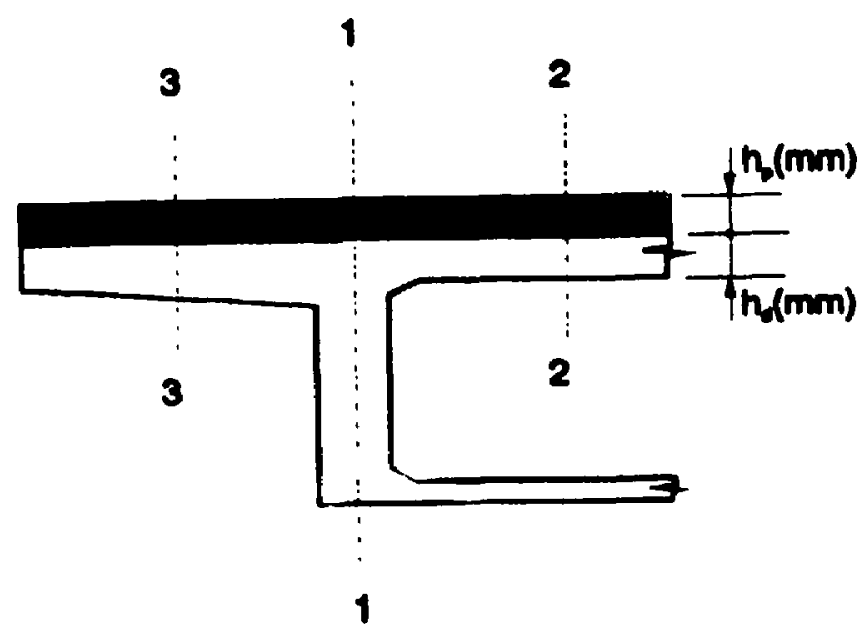

$h_{1}$ - thiokneas of black pavement

$h_{1}=$ thickneses of deck slab
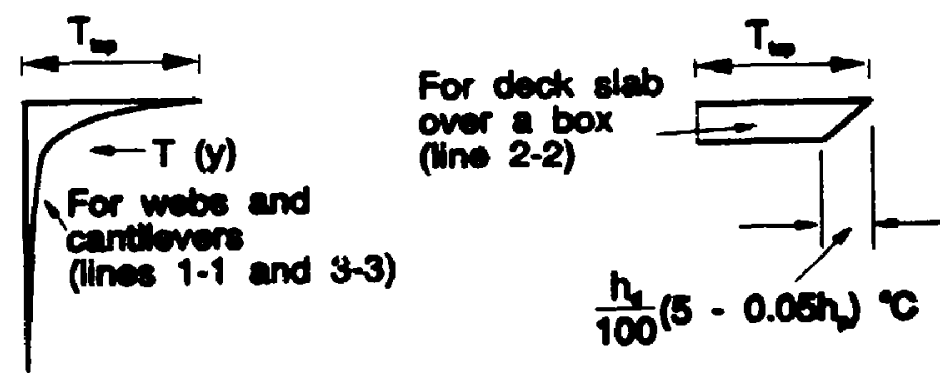

$T_{m}=32-0.2 h_{n}$

$T(y)=T_{-\infty}\left(\frac{y}{D} T^{-}\right.$

Typo 1

Type 2

D - espeth of the box olveler

Fig. A.4 Definition of types of temperature distribution. 

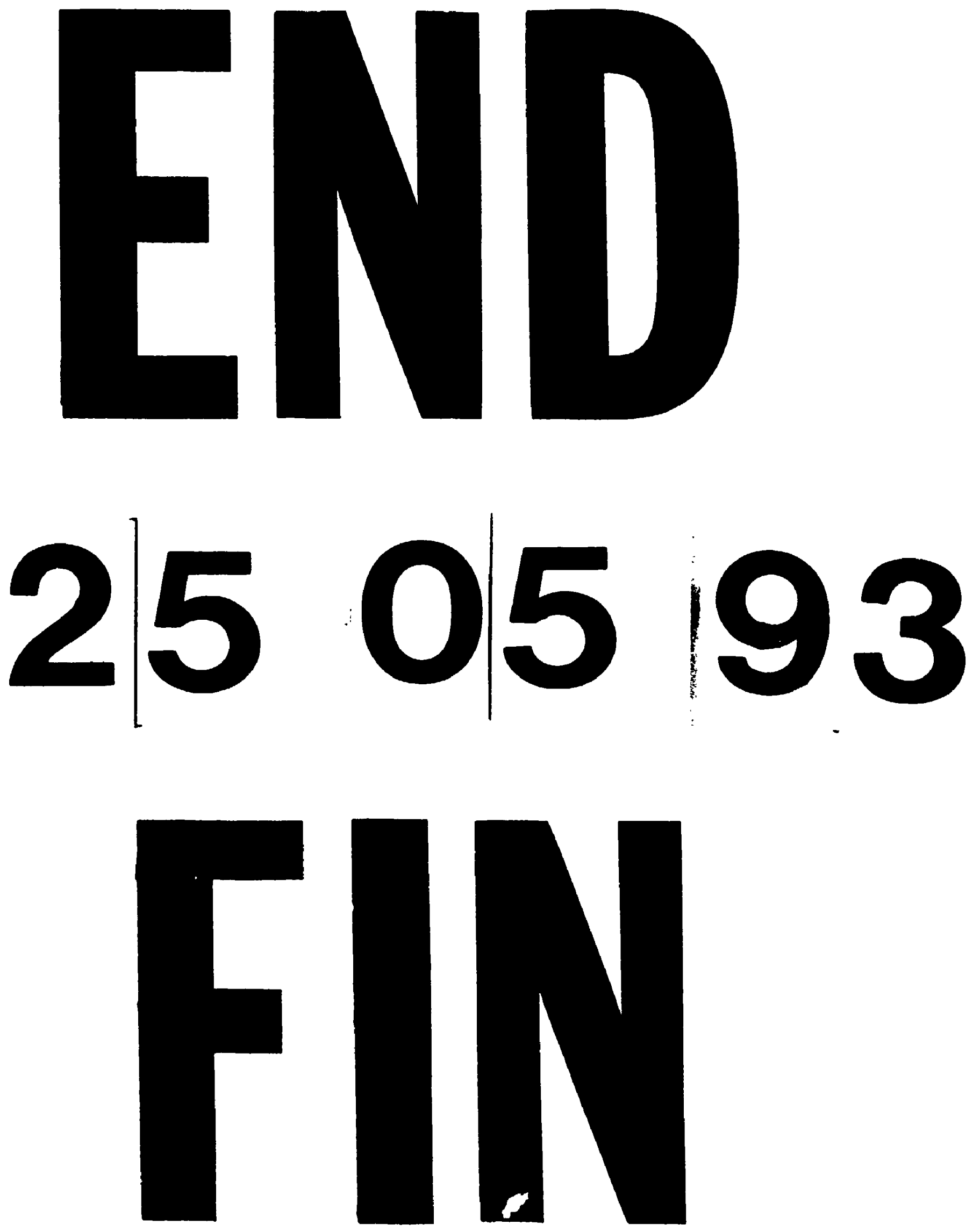